\title{
WestVirginiaUniversity
}

THE RESEARCH REPOSITORY @ WVU

Graduate Theses, Dissertations, and Problem Reports

2015

\section{Asphalt and Concrete Pavement Condition Analysis for the WVDOH}

Frank Peter Taverna III

Follow this and additional works at: https://researchrepository.wvu.edu/etd

\section{Recommended Citation}

Taverna III, Frank Peter, "Asphalt and Concrete Pavement Condition Analysis for the WVDOH" (2015). Graduate Theses, Dissertations, and Problem Reports. 6778.

https://researchrepository.wvu.edu/etd/6778

This Thesis is protected by copyright and/or related rights. It has been brought to you by the The Research Repository @ WVU with permission from the rights-holder(s). You are free to use this Thesis in any way that is permitted by the copyright and related rights legislation that applies to your use. For other uses you must obtain permission from the rights-holder(s) directly, unless additional rights are indicated by a Creative Commons license in the record and/ or on the work itself. This Thesis has been accepted for inclusion in WVU Graduate Theses, Dissertations, and Problem Reports collection by an authorized administrator of The Research Repository @ WVU. For more information, please contact researchrepository@mail.wvu.edu. 


\section{Asphalt and Concrete Pavement Condition Analysis for the WVDOH}

\section{Frank Peter Taverna III}

Thesis submitted to the College of Engineering and Mineral Resources at West Virginia University in partial fulfillment of the requirements for the degree of

Master of Science

In

Civil Engineering

Dr. John P. Zaniewski, Chair

Dr. John Quaranta

Dr. Avinash Unnikrishnan

Department of Civil and Environmental Engineering

Morgantown, West Virginia

March 2015

Key Words: Pavement Surface Rating, PSR, pavement condition, pavement surface distress, pavement type selection, 


\section{Abstract \\ Asphalt and Concrete Pavement Condition Analysis for the WVDOH Frank Peter Taverna III}

The objective of this research was to compare the condition of the asphalt and concrete sections of pavement on a section of US 35 for the West Virginia Division of Highways. In the process of completing this objective, existing procedures for pavement assessment were evaluated and an improved methodology for pavement condition evaluation, in terms of Pavement Surface Rating, PSR, was established. PSR uses observable surface distresses to quantify the condition of a pavement on a 0 to 100 scale. The PSR methodology includes surface distress data collection, a distress identification manual, and a PSR calculation procedure and spreadsheet. This process has been adopted by the WVDOH and is included in the special provision specification for pavements constructed using a warranted agreement.

Data from the WVDOH Pavement Management System, PMS, were used to evaluate pavement condition but ultimately deemed ineffective in a relatively small scale analysis. The methodology used to collect and analyze the PMS data was not compatible with the PSR methodology.

Deflection data gathered with a falling weight deflectometer were used to evaluate and compare the structural condition of the US 35 asphalt and concrete pavement sections. A screening of the deflection data on the concrete sections found the number of observations was an insufficient for meaningful analysis. The deflection data on the asphalt pavement sections were suitable for analysis. Since the WVDOH does not have an analysis methodology for deflection data, the research effort on this project focused on applying existing methodologies used for the analysis of deflection data. Due to the data problems, the analysis of the concrete sections was limited. The analysis of the asphalt sections was able to determine effective structural number for the asphalt sections. The analysis process is complex and several issues should be resolved to improve the ability of the WVDOH to use deflection analysis in a routine manner. 


\section{Table of Contents}

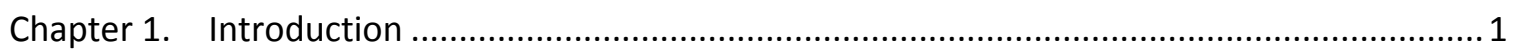

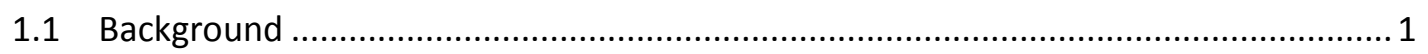

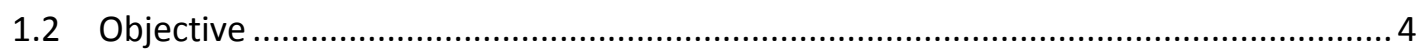

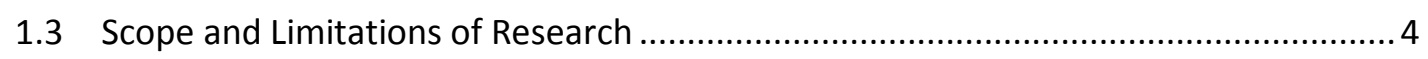

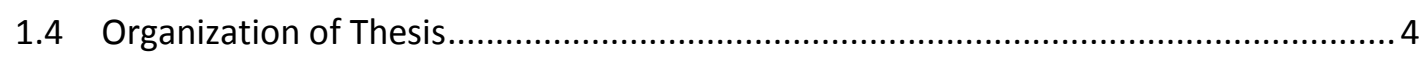

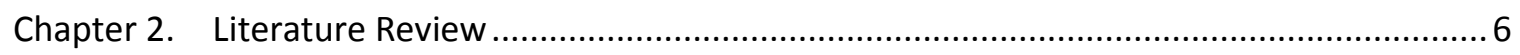

2.1 Pavement Surface Distress Indices and Rating Systems ........................................ 6

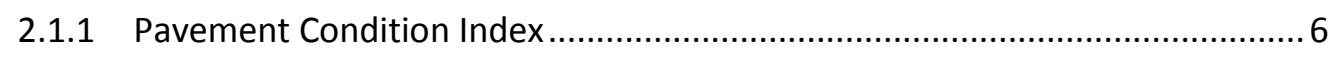

2.1.2 MnDOT Surface Rating ..................................................................... 12

2.1.3 WVDOH Pavement Surface Rating, PSR .................................................. 14

2.1.4 West Virginia DOH PMS Pavement Distress Evaluation.............................. 18

2.2 Deflection Testing and Analysis of Deflection Data ............................................... 21

2.2.1 Falling Weight Deflectometer and Backcalculation of Layer Moduli.............21

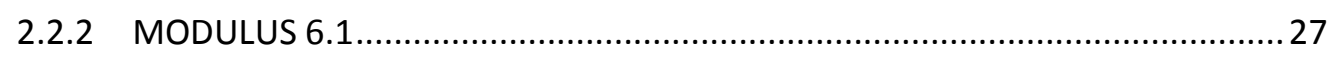

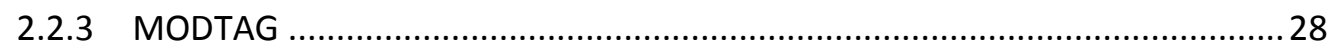

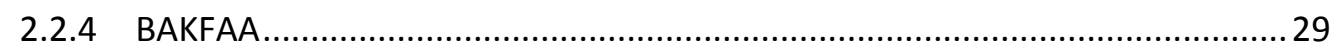

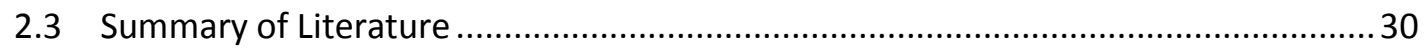

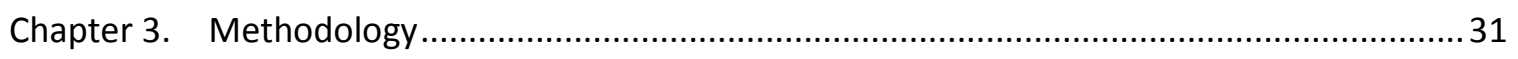

3.1 Pavement Surface Rating Data Collection and Analysis Process .............................. 31

3.1.1 Data Collection Process for Pavement Surface Rating ................................ 31

3.1.2 Data Analysis Process for Pavement Surface Rating ................................... 35

3.2 ARAN Surface Distress Data Collection and Comparison Process ............................... 39

3.3 Deflection Testing Data Collection and Analysis Process.......................................... 39

3.3.1 Deflection Data Collection ................................................................ 40 
3.3.2 Initial Screening of Deflection Data........................................................ 40

3.3.3 MODULUS 6.1 Deflection Data Analysis Process ........................................ 40

3.3.4 MODTAG Deflection Data Analysis Process ............................................... 42

3.3.5 BAKFAA Deflection Data Analysis Process …..................................................42

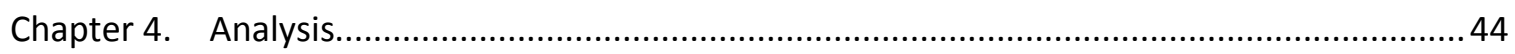

4.1 Analysis of Improvements in Pavement Surface Rating Process .............................. 44

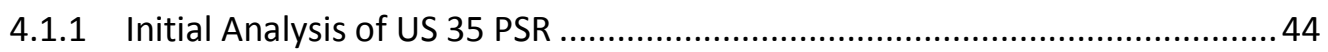

4.1.2 Refinement of PSR Calculation Procedure ...............................................46

4.2 Comparison Analysis of ARAN and Manual Survey Surface Distress Data ................ 51

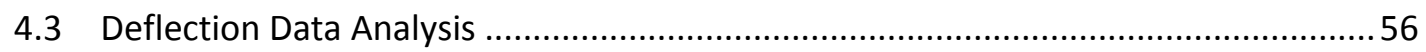

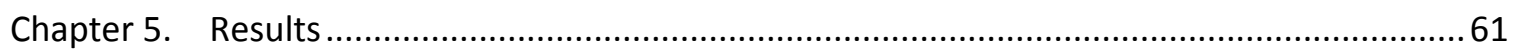

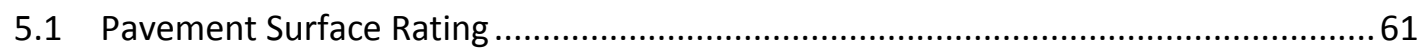

5.1.1 US 35 PSR Original 2012 Spec. Calculation................................................61

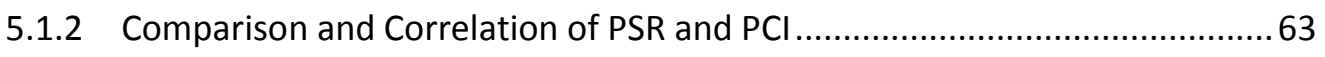

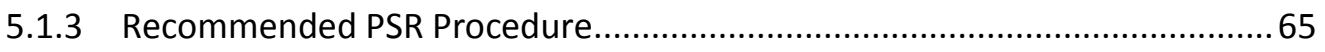

5.1.4 US 35 PSR with Recommended Method .................................................. 68

5.1.5 US 35 PSR Final Calculation vs. Original 2012 Spec. Calculation..................... 78

5.2 Comparison of ARAN and PSR Survey Surface Distress Data.................................... 84

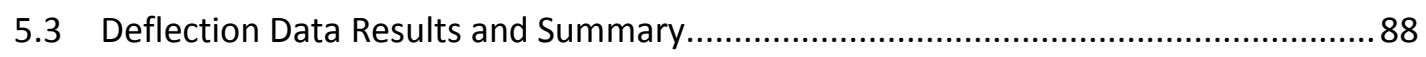

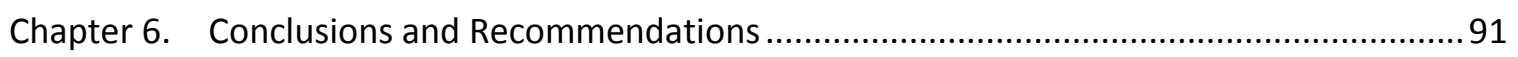

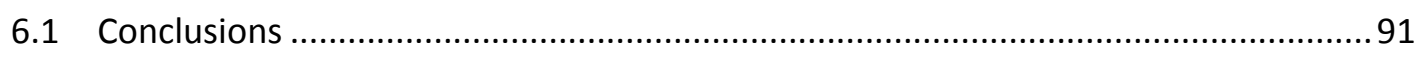

6.1.1 Pavement Surface Rating …........................................................... 91

6.1.2 Comparison of ARAN and PSR Survey Surface Distress Data ........................95

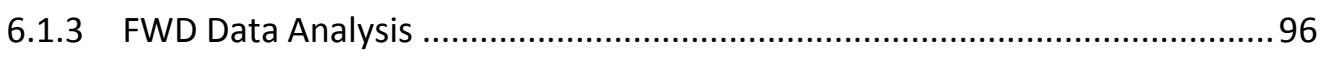

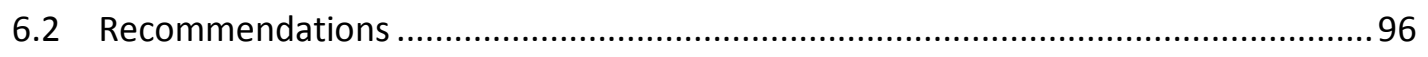

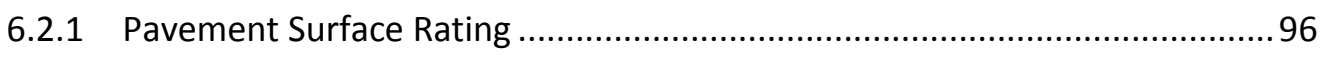




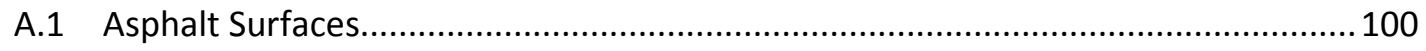

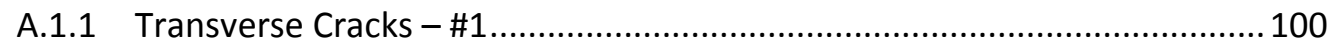

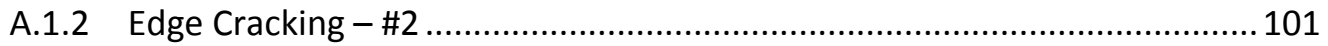

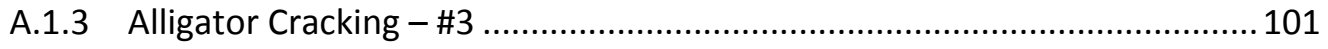

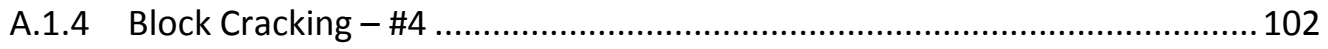

A.1.5 Longitudinal Cracking - \#5 ................................................................. 103

A.1.6 Longitudinal Joint Deterioration - \#6 ................................................... 103

A.1.7 Lane Edge Cracking/Deterioration - \#7 ............................................... 104

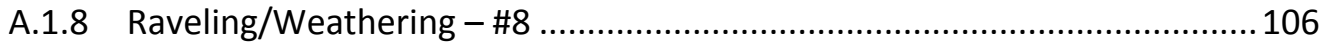

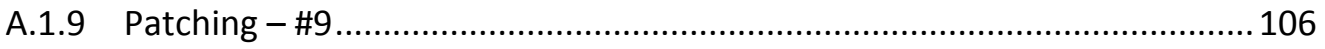

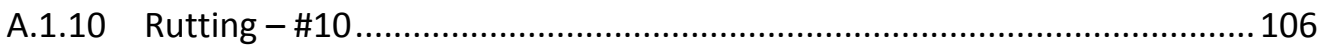

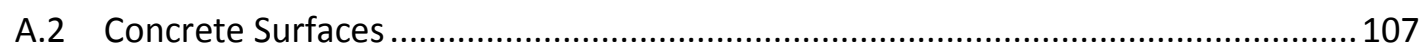

A.2.1 Transverse Cracks - \#11 .................................................................. 107

A.2.2 Transverse Joint Spalls - \#12 …........................................................ 108

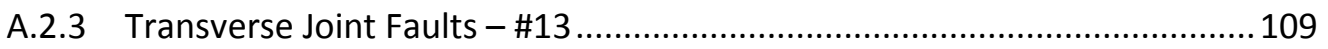

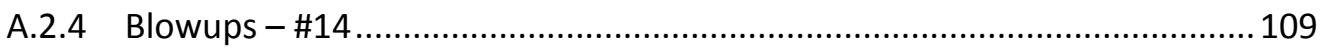

A.2.5 Longitudinal Cracking - \#15 ........................................................... 110

A.2.6 Longitudinal Joint Spalling - \#16 ........................................................... 110

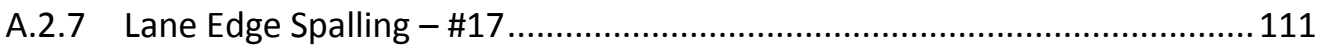

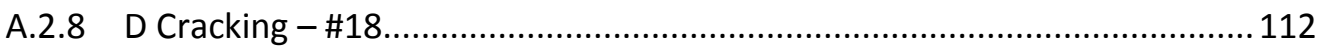

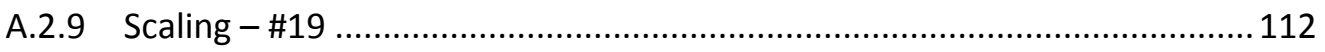

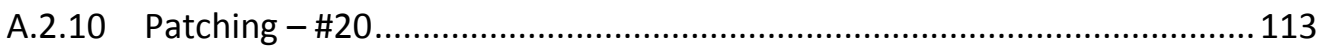

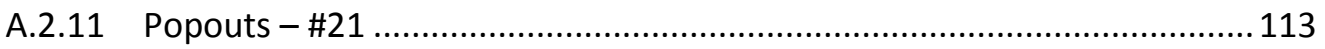


Appendix B. Deduct Curve Comparison

B.1 Deduct Comparison for Asphalt Surfaces ….................................................. 115

B.2 Deduct Comparison for Concrete Surfaces.................................................... 125

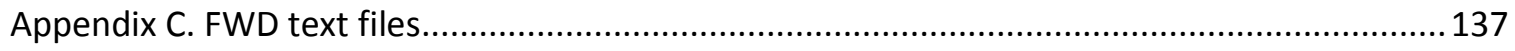

C.1 US 35 All Northbound Drops .FWD (.R80) File Format ......................................... 137

C.2 US 35 All Southbound Drops .FWD (.R80) File Format .......................................... 147

\section{List of Figures}

Figure 1: Location of US 35 Section Designated for Research Project................................................ 1

Figure 2: Example of a Flexible Pavement Condition Survey Data Sheet (ASTM D6433-11, 2011)............. 7

Figure 3: Example of Jointed Rigid Pavement Condition Survey Data Sheet (ASTM D6433-11, 2011) ........ 8

Figure 4: Example Deduct Value Curves (ASTM D6433-11, 2011) ........................................................ 9

Figure 5: Calculation of Corrected PCI Value-Flexible Pavement Example from Figure 2(ASTM D6433-11, 2011)

Figure 6: Corrected Deduct Value from Total Deduct Value and number of deducts " $q$ " for HMA (ASTM

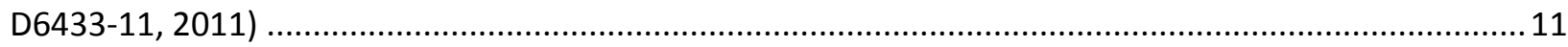

Figure 7: Standard PCI Rating Scale (after ASTM D6433-11, 2011) .................................................... 11

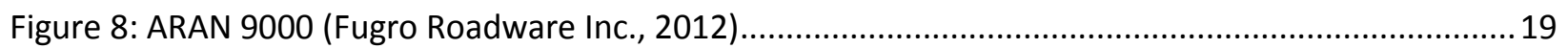

Figure 9: WVDOH DYNATEST Falling Weight Deflectometer Trailer on US 35 .......................................22

Figure 10: Falling Weight Deflectometer Deflection Basin (Cornell University, 2005)...........................23

Figure 11: Averaged graphical representation of temperature gradient within asphalt pavements

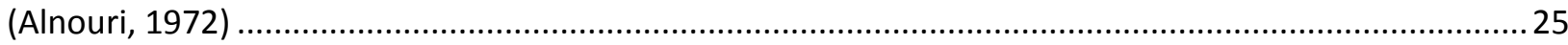

Figure 12: Asphalt Section Surface Distress Survey Diagram Segment ................................................. 33

Figure 13: Concrete Section Surface Distress Survey Diagram Segment................................................ 34

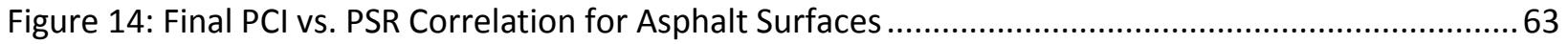

Figure 15: Final PCI vs. PSR Correlation for Concrete Surfaces............................................................. 64

Figure 16: Combination of PCI vs. PSR Correlations to Compare Asphalt and Concrete...........................64

Figure 17: Combined PCI vs. PSR Correlations for all Pavement Surfaces...............................................65

Figure 18: Blank Asphalt Surface Segment PSR Calculator .................................................................67

Figure 19: Blank Concrete Surface Segment PSR Calculator.................................................................68 
Figure 20: US 35 Asphalt Northbound Slow Lane Pavement Age vs. PSR …........................................ 71

Figure 21: US 35 Asphalt Northbound Slow Lane Mile Marker vs. PSR .................................................. 71

Figure 22: US 35 Asphalt Northbound Fast Lane Pavement Age vs. PSR ................................................. 72

Figure 23: US 35 Asphalt Northbound Fast Lane Mile Marker vs. PSR ................................................. 72

Figure 24: US 35 Asphalt Southbound Slow Lane Pavement Age vs. PSR ….......................................... 73

Figure 25: US 35 Asphalt Southbound Slow Lane Mile Marker vs. PSR …............................................... 73

Figure 26: US 35 Asphalt Southbound Fast Lane Pavement Age vs. PSR............................................... 74

Figure 27: US 35 Asphalt Southbound Fast Lane Mile Marker vs. PSR .................................................... 74

Figure 28: US 35 Concrete Northbound Slow Lane Pavement Age vs. PSR …......................................... 75

Figure 29: US 35 Concrete Northbound Slow Lane Mile Markers vs. PSR ….......................................... 75

Figure 30: US 35 Concrete Northbound Fast Lane Pavement Age vs. PSR ............................................. 76

Figure 31: US 35 Concrete Northbound Fast Lane Mile Marker vs. PSR ................................................ 76

Figure 32: US 35 Concrete Southbound Slow Lane Pavement Age vs. PSR .............................................. 77

Figure 33: US 35 Concrete Southbound Slow Lane Mile Marker vs. PSR ….............................................77

Figure 34: US 35 Concrete Southbound Fast Lane Pavement Age vs. PSR ............................................. 78

Figure 35: US 35 Concrete Southbound Fast Lane Mile Marker vs. PSR ….......................................... 78

Figure 36: Asphalt Northbound Slow Lane Survey 5 - 2012 Spec. vs 2015 Spec. .................................... 80

Figure 37: Asphalt Northbound Fast Lane Survey 5 - 2012 Spec. vs 2015 Spec. ....................................... 80

Figure 38: Asphalt Southbound Slow Lane Survey 5 - 2012 Spec. vs 2015 Spec. .................................... 81

Figure 39: Asphalt Southbound Fast Lane Survey 5 - 2012 Spec. vs 2015 Spec. .................................... 81

Figure 40: Concrete Northbound Slow Lane Survey 5 - 2012 Spec. vs 2015 Spec....................................82

Figure 41: Concrete Northbound Fast Lane Survey 5 - 2012 Spec. vs 2015 Spec..................................... 83

Figure 42: Concrete Southbound Slow Lane Survey 5 - 2012 Spec. vs 2015 Spec.................................... 83

Figure 43: Concrete Southbound Fast Lane Survey 5 - 2012 Spec. vs. 2015 Spec..................................... 84

Figure 44: Structural Cracking Index - Manual Survey Data vs. PMS Data Comparison ............................ 85

Figure 45: Environmental Cracking Index - Manual Survey Data vs. PMS Data Comparison ...................... 85

Figure 46: Net Cracking Index - Manual Survey Data vs. PMS Data Comparison ..................................... 86

Figure 47: Rut Depth Index - Manual Survey Data vs. PMS Data Comparison ......................................... 86

Figure 48: Joint Condition Index - Manual Survey Data vs. PMS Data Comparison .................................. 87

Figure 49: Concrete Slab Index - Manual Survey Data vs. PMS Data Comparison ..................................... 87

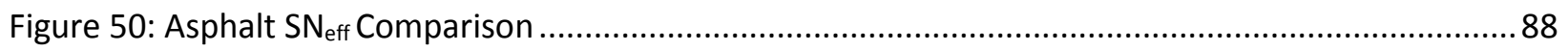

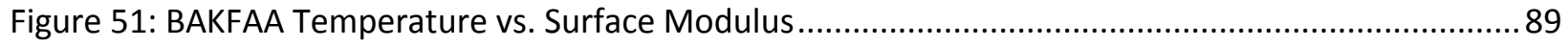




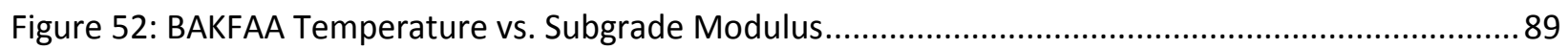

Figure 53: Graphical Comparison of PCl and 2015 PSR Ranges for Qualitative Descriptors ..................... 92

Figure 54: Average PSR Comparison: Asphalt vs. Concrete ............................................................... 93

Figure 55: Asphalt Average PSR Comparison: Fast Lane vs. Slow Lane ................................................94

Figure 56: Concrete Average PSR Comparison: Fast Lane vs. Slow Lane...............................................94

Figure 57: Fast Lane Average PSR Comparison: Asphalt vs. Concrete .................................................... 95

Figure 58: Slow Lane Average PSR Comparison: Asphalt vs. Concrete.................................................. 95

Figure 59:Transverse Cracks Low Severity Deduct Curve Comparison ................................................115

Figure 60: Transverse Cracks Medium Severity Deduct Curve Comparison..........................................116

Figure 61: Transverse Cracks High Severity Deduct Curve Comparison ................................................116

Figure 62: Edge Cracking Low Severity Deduct Curve Comparison ..................................................... 117

Figure 63: Edge Cracking Medium Severity Deduct Curve Comparison ................................................117

Figure 64: Edge Cracking High Severity Deduct Curve Comparison .....................................................118

Figure 65: Alligator Cracking Low Severity Deduct Curve Comparison ................................................ 118

Figure 66: Alligator Cracking Medium Severity Deduct Curve Comparison ..........................................119

Figure 67: Alligator Cracking High Severity Deduct Curve Comparison................................................ 119

Figure 68: Block Cracking Low Severity Deduct Curve Comparison .................................................... 120

Figure 69: Block Cracking Medium Severity Deduct Curve Comparison ............................................... 120

Figure 70: Block Cracking High Severity Deduct Curve Comparison..................................................... 121

Figure 71: Longitudinal Cracking Low Severity Deduct Curve Comparison ......................................... 121

Figure 72: Longitudinal Cracking Medium Severity Deduct Curve Comparison ...................................... 122

Figure 73: Longitudinal Cracking High Severity Deduct Curve Comparison ............................................ 122

Figure 74: Raveling/Weathering Deduct Curve Comparison ............................................................... 123

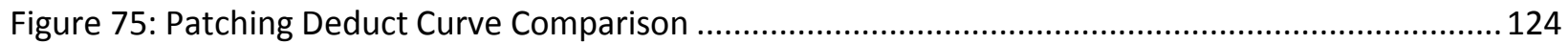

Figure 76: Rutting Low Severity Deduct Curve Comparison ............................................................ 125

Figure 77: Rutting High Severity Deduct Curve Comparison .............................................................. 125

Figure 78: Transverse Cracks Low Severity Deduct Curve Comparison ................................................. 126

Figure 79: Transverse Cracks Medium Severity Deduct Curve Comparison........................................... 126

Figure 80: Transverse Cracks High Severity Deduct Curve Comparison ................................................ 127

Figure 81: Transverse Joint Spalls Low Severity Deduct Curve Comparison ......................................... 128

Figure 82: Transverse Joint Spalls Medium Severity Deduct Curve Comparison .................................. 128

Figure 83: Transverse Joint Spalls High Severity Deduct Curve Comparison........................................ 129 


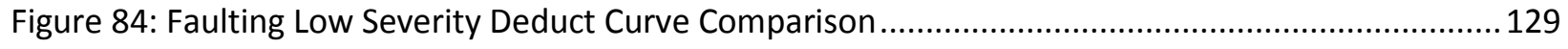

Figure 85: Faulting Medium Severity Deduct Curve Comparison.........................................................130

Figure 86: Faulting High Severity Deduct Curve Comparison ............................................................. 130

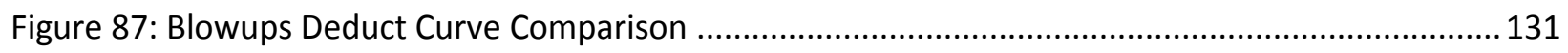

Figure 88: Longitudinal Cracking Low Severity Deduct Curve Comparison .......................................... 132

Figure 89: Longitudinal Cracking Medium Severity Deduct Curve Comparison ..................................... 132

Figure 90: Longitudinal Cracking High Severity Deduct Curve Comparison .......................................... 133

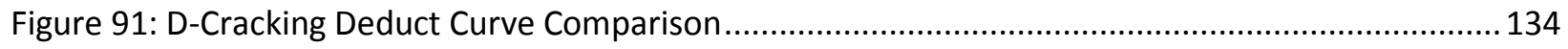

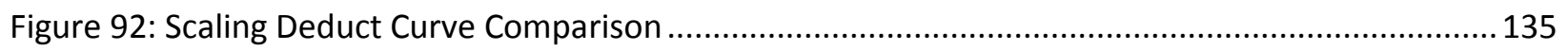

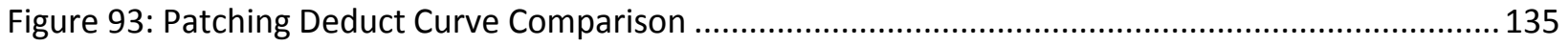

Figure 94: Popouts Deduct Curve Comparison .................................................................................. 136

\section{List of Tables}

Table 1: List of one-tenth mile test sections by start/end mile post ...................................................... 3

Table 2: HMA Surface Pavement Distress Types and Measurements (Office of Materials and Road

Research Pavement Management Unit, 2003)

Table 3: Jointed Concrete Pavement Distress Types and Measurements (Office of Materials and Road

Research Pavement Management Unit, 2003)......

Table 4: HMA Pavement SR Weight Factors (Office of Materials and Road Research Pavement

Management Unit, 2003).

Table 5: Jointed Concrete Pavement SR Weight Factors (Office of Materials and Road Research

Pavement Management Unit, 2003)

Table 6: Total Weighted Percent or Total Weighted Distress Conversion to SR (Office of Materials and

Road Research Pavement Management Unit, 2003)

Table 7: PSR Asphalt Distresses, severity levels, and units (Section 490 Nine Year Pavement Performance

Criteria)

Table 8: PSR Concrete Distresses, severity levels, and units (Section 490 Nine Year Pavement

Performance Criteria, 2012)

Table 9: PSR Asphalt Distress Weight Factors (Section 490 Nine Year Pavement Performance Criteria, 2012)

Table 10: PSR Concrete Distress Weight Factors (Section 490 Nine Year Pavement Performance Criteria, 2012) 17 
Table 11: Example of Incentive Performance Criteria for Bonus and Penalty (Section 490 Nine Year

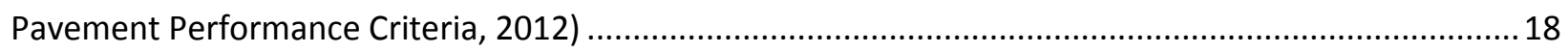

Table 12: Longitudinal Cracking within NB 12.6-12.7 (HMA Section).................................................... 36

Table 13: Transverse Spalled Joints within NB 9.1-9.2 (PCC Section) ...................................................... 37

Table 14: Post Survey 3 PSR Calculator for Slow Lane of NB 13.2-13.3 (HMA Section) ............................ 38

Table 15: Post Survey 3 PSR Calculator for Slow Lane of NB 9.4-9.5 (PCC Section) .................................. 38

Table 16: Summary of WVDOH PMS Distress Data for US 35 Northbound Slow Lane Asphalt Section......52

Table 17: Summary of WVDOH PMS Distress Data for US 35 Northbound Slow Lane Concrete Section ...52

Table 18: WVDOH PMS Legend for Distress Data as provided with Distress Summary ...........................53

Table 19: Variation between PSI calculated from IRI and PSI provided ...............................................55

Table 20: Inconsistencies between WVDOH PMS and Section 490, Special Provision distress definition

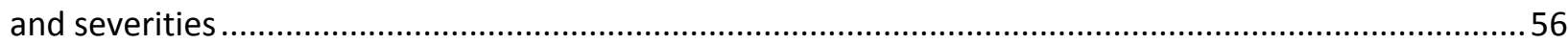

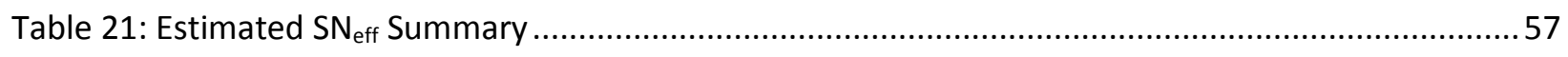

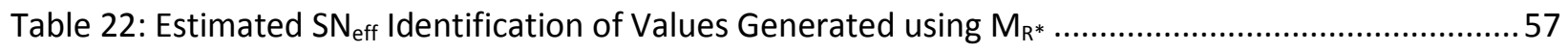

Table 23: Summary of Asphalt Section SNeff Values at Each Test Location with Addition of Cut/Fill

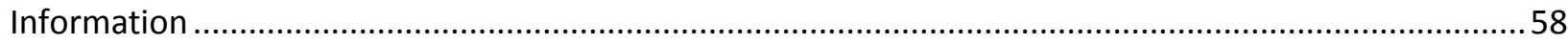

Table 24: Reorganization of Table 23 by Cuts and Fills.......................................................................59

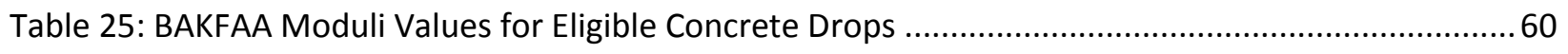

Table 26: Initial PSR Calculation for Northbound Asphalt Section ........................................................61

Table 27: Initial PSR Calculation for Northbound Concrete Section .......................................................61

Table 28: Initial PSR Calculation for Southbound Asphalt Section .........................................................62

Table 29: Initial PSR Calculation for Southbound Concrete Section ......................................................62

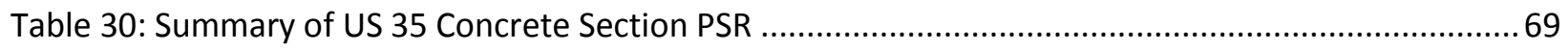

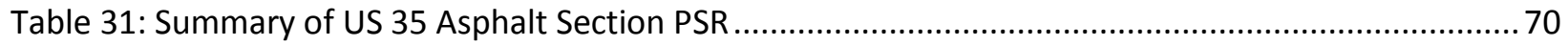

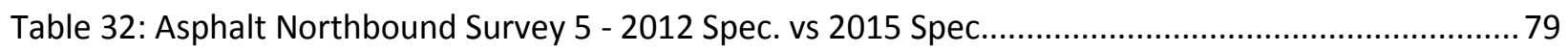

Table 33: Asphalt Southbound Survey 5 - 2012 Spec. vs 2015 Spec......................................................... 79

Table 34: Concrete Northbound Survey 5 - 2012 Spec. vs 2015 Spec.................................................... 82

Table 35: Concrete Southbound - Survey 5 - 2012 Spec. vs 2015 Spec.................................................. 82

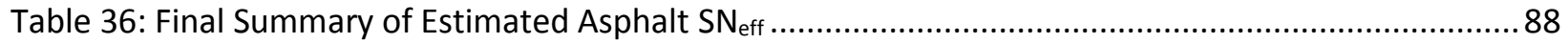

Table 37: Asphalt and Concrete Average Subgrade Modulus Values......................................................90

Table 38: Comparison of PCl and 2015 PSR Ranges for Qualitative Descriptors ..................................... 91 


\section{Chapter 1. Introduction}

\subsection{Background}

Slightly over 7 miles of US 35 in Mason County, south of Point Pleasant, West Virginia were paved in October 2010 and were identified as a potential subject for development of a warranty specification and additional research for the West Virginia Department of Transportation Division of Highways. The approximate start and end points of the test section are the two intersections of Kanawha Valley Road and US 35. These are located at mile post 9.0 on the south end and 16.5 on the north end of the test section. This stretch of US 35 is a divided highway with 2 lanes in the northbound and southbound directions. It is a common truck route between Charleston, West Virginia and regions of Ohio. At mile post 12.5, which is the overpass bridging Upper Fivemile Creek and Arlee-Couch Road, there is a border between pavement types. Everything south of this point is portland cement concrete constructed by Kokosing Construction Company. Everything north of this point is asphalt pavement laid by West Virginia Paving and Bizzack Construction Company. Figure 1 identifies the section of US 35 designated for this research project.

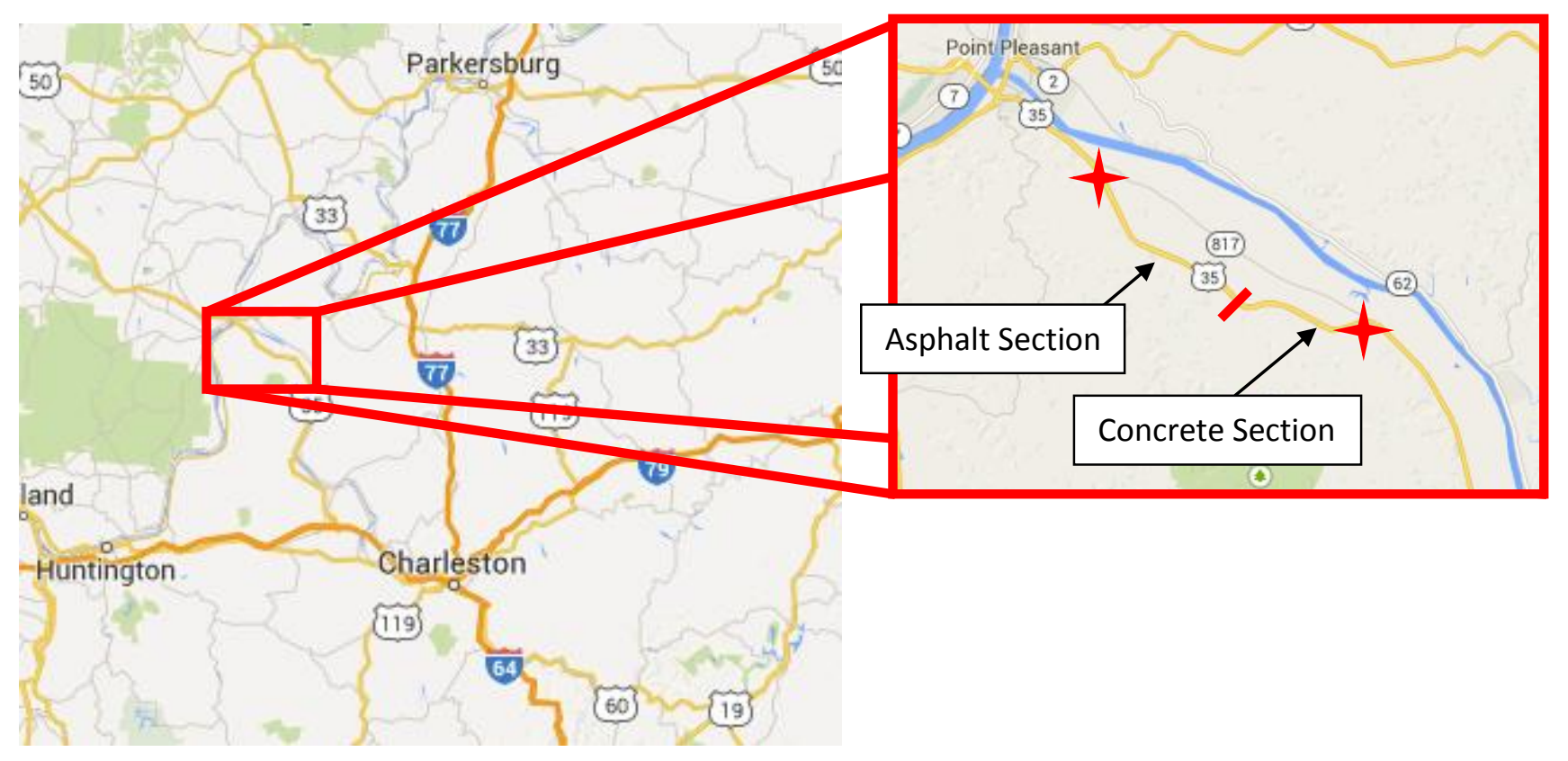

Figure 1: Location of US 35 Section Designated for Research Project

Of the 7-plus miles of highway section (northbound and southbound), 16 one-tenth mile sections in both the northbound and southbound directions ( 32 total) were selected as a representative 
sample (shown in Table 1). There are three overpasses and three intersections within the entire section. All overpasses are concrete slab bridges with small sections paved in asphalt approaching and leaving the bridges. Within the concrete test sections, there are two cases where the northbound and southbound test section mile posts do not match. Test sections were shifted to avoid overpasses in these instances. Of the three intersections, none are significant stopping points for any trucks, so essentially all heavy traffic travels the length of the test section. Of the 16 one-tenth mile sections in each direction, nine were paved entirely in asphalt and the remaining seven in concrete. The surface materials, directions, and start/finish mile posts (in order of travel direction) are shown in Table 1.

According to as-built drawings, from surface down, asphalt pavement cross-sections consist of:

- $\quad 1.5^{\prime \prime}$ Superpave HMA $9.5 \mathrm{~mm}$ NMAS

- 2.25" Superpave HMA 19 mm NMAS

- 9" Superpave HMA 37.5 mm NMAS (Two 4.5" lifts)

- 4" open graded free draining base

- Subgrade soil

Per the WVDOH Design Directive manual, the Structural Number of this pavement is approximately 6.7 .

From surface down, PCC cross-sections consist of:

- $10^{\prime \prime}$ depth concrete slab

- 6" open graded free draining base

- Subgrade soil

Since there are two lanes in each direction of the divided highway, they will be referred to as the fast and slow lane throughout. If one were to be driving on the highway, the fast lane would be to the left of the slow lane. (Right and left lanes were not used as identifiers to eliminate confusion in data collection and analysis where travel direction is not always obvious.) Lanes in all sections are approximately 11.5 feet wide with longitudinal construction joints immediately to the slow lane side of the lane divider line. There is also a longitudinal construction joint between the slow lane and the shoulder in all sections. In the asphalt sections the paving width extends approximately 1.5 feet outside into the shoulder of the fast lane. In the concrete sections there is a longitudinal construction joint immediately outside of the fast lane and an additional slab approximately 1.5 feet wide. Concrete slab lengths are approximately 15 feet. In all one-tenth mile concrete test sections, there are 35 full slabs per lane and one partial slab. 


\begin{tabular}{|l|l|l|}
\cline { 2 - 3 } \multicolumn{1}{c|}{} & Southbound & Northbound \\
\hline \multirow{5}{*}{ Asphalt } & $16.5-16.4$ & $16.4-16.5$ \\
& $16.1-16.0$ & $16.0-16.1$ \\
& $15.5-15.4$ & $15.4-15.5$ \\
& $15.3-15.2$ & $15.2-15.3$ \\
& $14.7-14.6$ & $14.6-14.7$ \\
& $14.5-14.4$ & $14.4-14.5$ \\
& $13.9-13.8$ & $13.8-13.9$ \\
& $13.3-13.2$ & $13.2-13.3$ \\
& $12.7-12.6$ & $12.6-12.7$ \\
\hline \multirow{5}{*}{ Sections } & $12.35-12.25$ & $12.2-12.3$ \\
& $11.8-11.7$ & $11.7-11.8$ \\
& $11.5-11.4$ & $11.4-11.5$ \\
& $10.9-10.8$ & $10.8-10.9$ \\
& $10.5-10.4$ & $10.4-10.5$ \\
& $9.5-9.4$ & $9.4-9.5$ \\
& $9.3-9.2$ & $9.1-9.2$ \\
\hline
\end{tabular}

Table 1: List of one-tenth mile test sections by start/end mile post

Manual surveys were performed in a joint effort by WVDOH employees and WVU graduate student researchers. Within each of the 32 test sections, fast and slow lanes existed which were surveyed simultaneously. The surveys were performed during May 2012, October 2012, April 2013, September 2013, and May 2014. For the remainder of this report, these will be referred to as Surveys 1 through 5 respectively.

During the manual survey the observed distresses were mapped onto data collection forms using the procedures described in Appendix A. These manual surveys generally consisted of teams of 2 to 4 people walking each of the one-tenth mile sections and drawing the observed distresses to scale on sheets of grid paper. The papers from the first survey were saved and used again for each subsequent survey. Additions to observed distresses were made in a different color each time. After each survey, observed distresses were tallied and a Pavement Surface Rating, PSR, was calculated for each section. PSR is a scale for quantifying the condition of a pavement based on observed surface distresses.

Initially, a PSR was computed per the April 19, 2012 version of "West Virginia Department of Transportation Division of Highways Special Provision, Section 490: Nine Year Pavement Performance Criteria." The purpose of the Special Provision document is to establish definitions and guidelines for performing surveys so PSR can be calculated. The pavement performance criteria described assures the 
quality of warranted pavements and protects the Division of Highways from specific distresses. PSR calculation is described in detail in Chapter 2.

In addition to the manual surveys, twice during the pavements life, a falling weight deflectometer (FWD) was used and deflection data were collected within the slow lane of all sections. These FWD tests took place in May 2012 and July 2014. The first was excluded from all analysis due to inconsistencies in sensor labeling, inability to identify location of drops, and excessive amounts of nondecreasing deflections.

Also surface distress data collected by an Automatic Road Analyzer, ARAN, for the northbound slow lane were available from the WVDOH Pavement Management System. These data and their analyses are described in Chapter 2.

\subsection{Objective}

The objective of this research was to compare asphalt and concrete pavements on US 35. Both pavement distress condition and deflection data were available for this comparison.

\subsection{Scope and Limitations of Research}

The scope of the research is the section of US 35 between mile posts 9.0 and 16.5. The data available to accomplish this objective were limited to the surface distress data collect for the Pavement Management System, periodic manual surveys, and the deflection data collected with the FWD.

\subsection{Organization of Thesis}

This thesis is organized into six chapters. Following this introduction, Chapter 2 contains a summary of three relevant pavement surface distress index/rating systems, background to a portion of the WVDOH Pavement Management System, and finally background for the Falling Weight Deflectometer as well as the procedure for backcalculating pavement layer moduli and three relevant computer programs which assist with this. Chapter 3 discusses the research methodologies used to collect data and assess the pavement condition using both surface distress and deflection data. Chapter 4 describes the analysis in detail which led to the results contained within Chapter 5 . Finally Chapter 6 discusses the relevant conclusions and a few recommendations which were made based on the findings. Additionally three appendices are included. Appendix A is a complete Distress Identification Manual which was developed to aid this research and will be subsequently used in combination with a specification for calculating Pavement Surface Rating for the WVDOH. Appendix B a comprehensive 
analysis of the deduct curves associated with Pavement Condition Index. This analysis was used for the development of the PSR procedure. Appendix C contains the deflection data collected by the Falling Weight Deflectometer and use by the three computer programs to backcalculate pavement layer moduli and other information. 


\section{Chapter 2. Literature Review}

A wide variety of pavement surface distress survey methods and associated indices are available in the literature. The following literature review is limited to four methods that are directly applicable to this research.

Similarly, the review of deflection measurement and analysis focus on the type of deflection device used by the WVDOH. Three backcalculation analysis methods are reviewed that represent the state of the practice.

\subsection{Pavement Surface Distress Indices and Rating Systems}

Four methods for quantifying surface distresses were reviewed for the purpose of this research. The first is Pavement Condition Index, $\mathrm{PCl}$, which was developed and influenced by the U.S. Army Corps of Engineers and the Washington State Department of Transportation, WSDOT, in the 1960's and 70's. The second is Surface Rating, SR, a procedure developed by Minnesota Department of Transportation, MnDOT. The next method reviewed is the 2012 Pavement Surface Rating, PSR, developed by the West Virginia Department of Transportation. It was derived from the MnDOT's Surface Rating. The method for computing PSR was refined during this research to meet the specific needs of the WVDOH. The final method is the pavement stress evaluation used for the WVDOH PMS.

\subsubsection{Pavement Condition Index}

ASTM D6433-11 “Standard Practice for Roads and Parking Lots Pavement Condition Index (PCI) Survey" provides a detailed approach to calculating $\mathrm{PCl}$. This condition index is based on a deduct value system which was developed originally by LeClerc and Marshall for the Washington State Department of Transportation in 1971. Shahin, with his team from the U.S. Army Corps of Engineers Construction Engineering Research Laboratory, further developed PCI for the PAVER system (as cited in Jackson, 2008).

"The $\mathrm{PCl}$ is a numerical index, ranging from 0 for a completely failed pavement to 100 for a pavement in perfect condition" (Shahin, 2005). Simply put, a pavement theoretically starts with a PCl of 100. It is then surveyed by trained inspectors and deductions are applied to the $\mathrm{PCl}$ based on the findings of the inspectors to determine the overall distress related condition of the pavement.

The distress is converted into a percentage based on the amount of test section affected. For the pavement condition survey, distress types are defined for asphalt and concrete surfaces. Severity 
levels are defined for each distress type, and the method for measuring the extent of the distress is described. Figure 2 and Figure 3 are examples of completed survey forms for asphalt (flexible) and concrete (rigid) pavements. The process for computing PCl for asphalt pavements is described below. The process for computing $\mathrm{PCl}$ for concrete pavements is similar.

The extent of each distress type and severity combination is tallied by total area affected. This is shown in the Distress Severity, Quantity, and Total column of Figure 2. For example, in the first row, the $1 \mathrm{~L}$ in column 1 indicates alligator cracking at low severity with a total extent of 13 square feet. For distresses that are measured in linear feet, such as longitudinal or transverse cracking, the width of affected area is assumed to be one foot. The "SKETCH" in Figure 2 shows there are 2500 square feet of surveyed test section. Equation 1 shows the density (\%) calculation for this case.

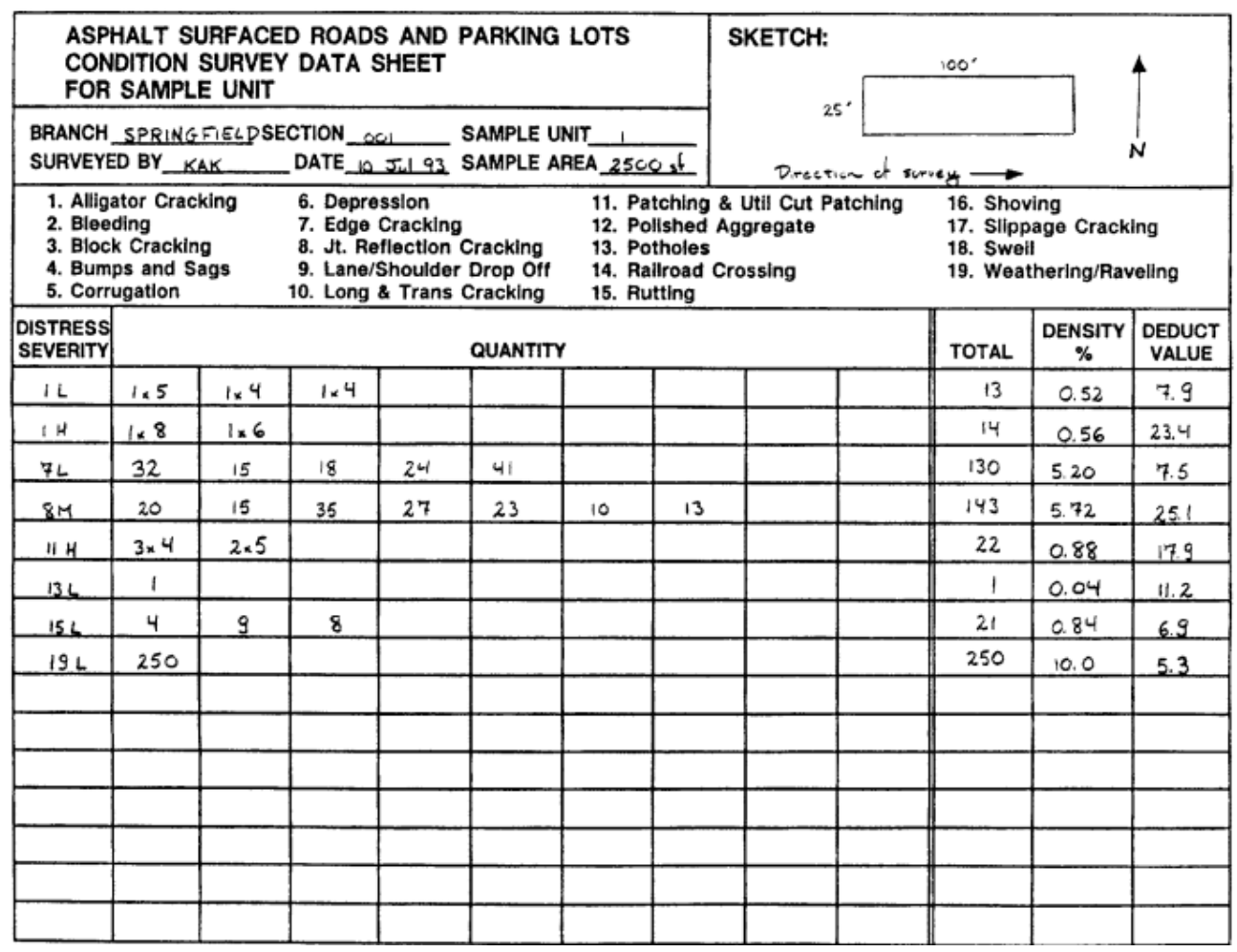

Figure 2: Example of a Flexible Pavement Condition Survey Data Sheet (ASTM D6433-11, 2011) 


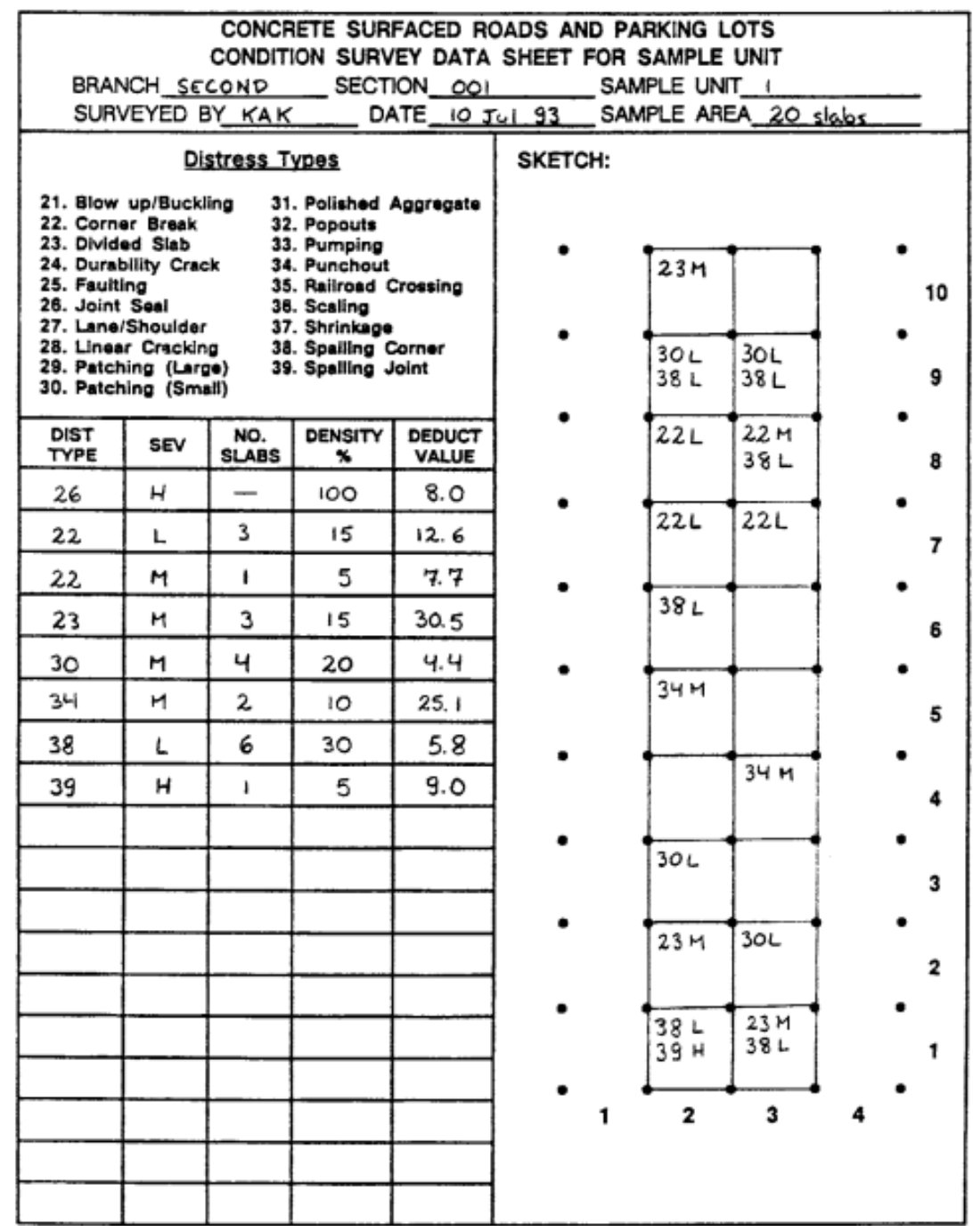

Figure 3: Example of Jointed Rigid Pavement Condition Survey Data Sheet (ASTM D6433-11, 2011)

$\frac{\text { Total Quantity of Individual Distress at given Severity }}{\text { Total Area Surveyed }} * 100=\frac{13}{2500} * 100=0.52 \%$

Next, the Deduct Value in the last column of Figure 2 is determined from a deduct value curve based on a distress types severity and density in percent. For example, from Figure 2, the Distress Severity labeled " $15 \mathrm{~L}$," corresponding to low severity rutting, was found to have a density of $0.84 \%$. In Figure 4 (a), the deduct value curve for rutting, low severity " $L$ " Rutting observed at a density of $0.84 \%$ has a Deduct Value of approximately 6.9, as shown in the right-most column of Figure 2. 


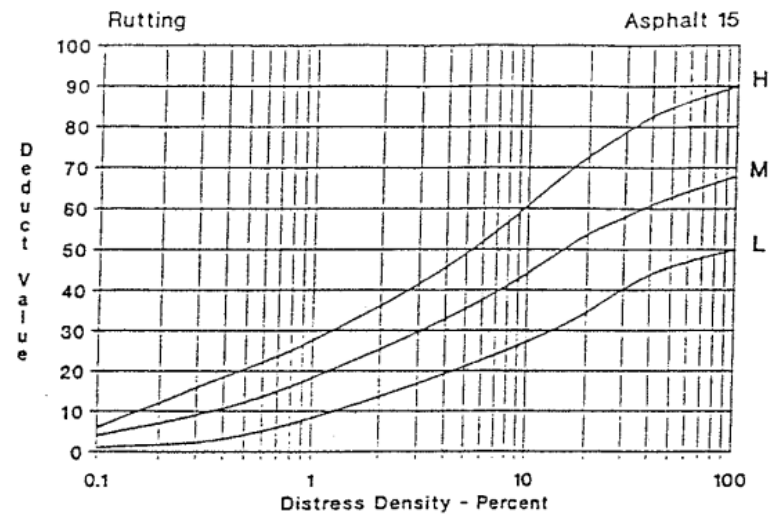

(a)

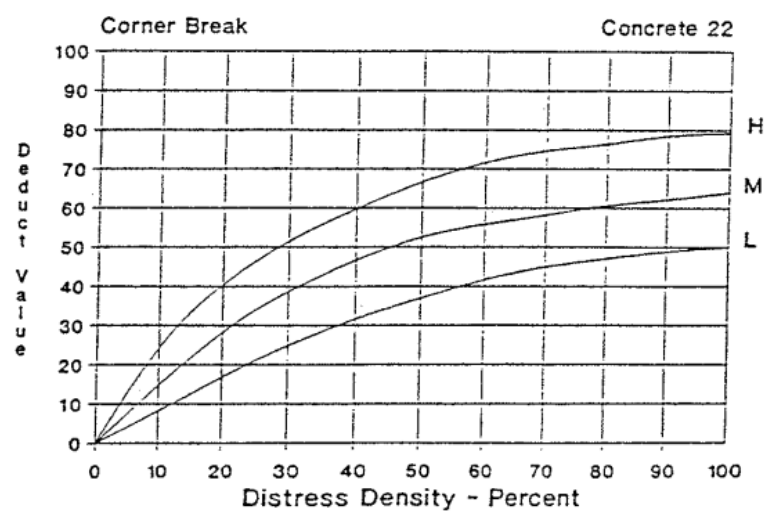

(b)

Figure 4: Example Deduct Value Curves (ASTM D6433-11, 2011)

The $\mathrm{PCl}$ is computed through an iterative analysis of the deduct values for the distress severity combinations. First, the maximum number of combinations that will be considered is computed as:

$m=1+\left(\frac{9}{98}\right) *(100-M a x D V)$

where:

$\mathrm{m} \quad=$ the allowable number of deducts including fractions (less than or equal to ten) MaxDV = the maximum deduct value observed

The value of $m$ is truncated to determine the number of whole deduct values that will be used for the corrected deduct value calculation. For the data in Figure $5, m=7.9$ so seven deduct values are transferred from Figure 2 to Figure 4, and arranged in descending order. The eighth and lowest deduct value from Figure 2 is adjusted by the decimal fraction from the $m$ calculation, e.g. the eighth largest deduct value from Figure 2, 5.3, is multiplied by 0.9 to compute the deduct value recorded in Figure 4.

The next step is to determine the corrected deduct value using Figure 6 . The value in the Total column of Figure 5 is used on the abscissa of Figure 6, Total Deduct Value. The total number of distresses with Total Deduct greater than 2 is " $q$ " and is used to select the curve on Figure 6 to determine the Corrected Deduct Value on the ordinate of Figure 6.

From there, a sequential approach is used to calculate the Maximum Corrected Deduct Value, Max CDV. In each step, the lowest deduct greater than 2 is reduced to 2 , q is reduced by 1 , Total is 
recalculated, and CDV is determined from Figure 6 . This process is repeated until q equals $1 . \mathrm{PCl}$ for the test section is calculated by subtracting the Maximum CDV from 100 as shown in Figure 5 . In this example, the Maximum CDV occurred on the first step of the analysis. However, depending on the relative magnitude of the Deduct Values this is not always the case.

Figure 7 provides qualitative descriptors for $\mathrm{PCl}$ ranges.

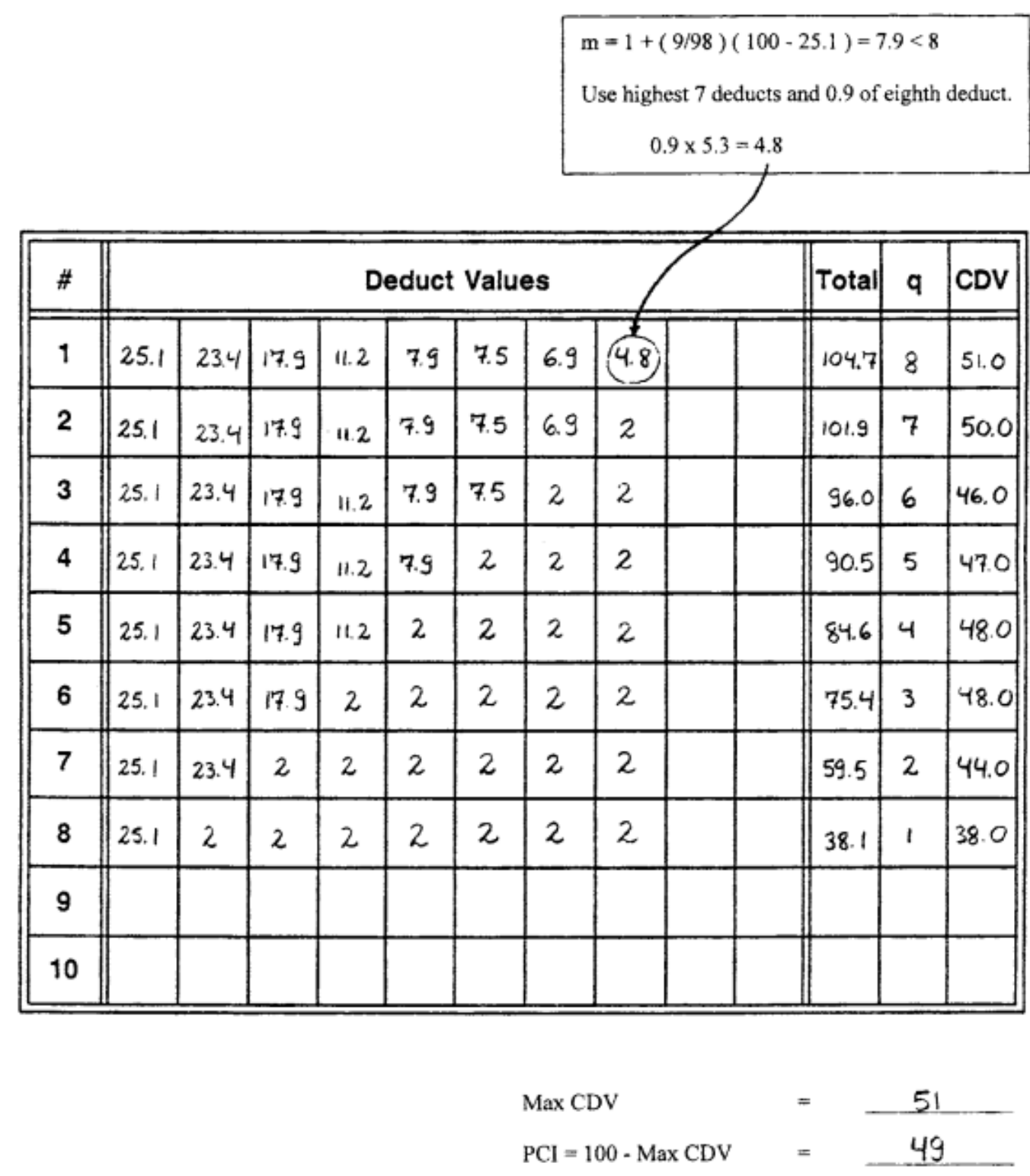

Figure 5: Calculation of Corrected PCI Value-Flexible Pavement Example from Figure 2(ASTM D6433-11, 2011) 


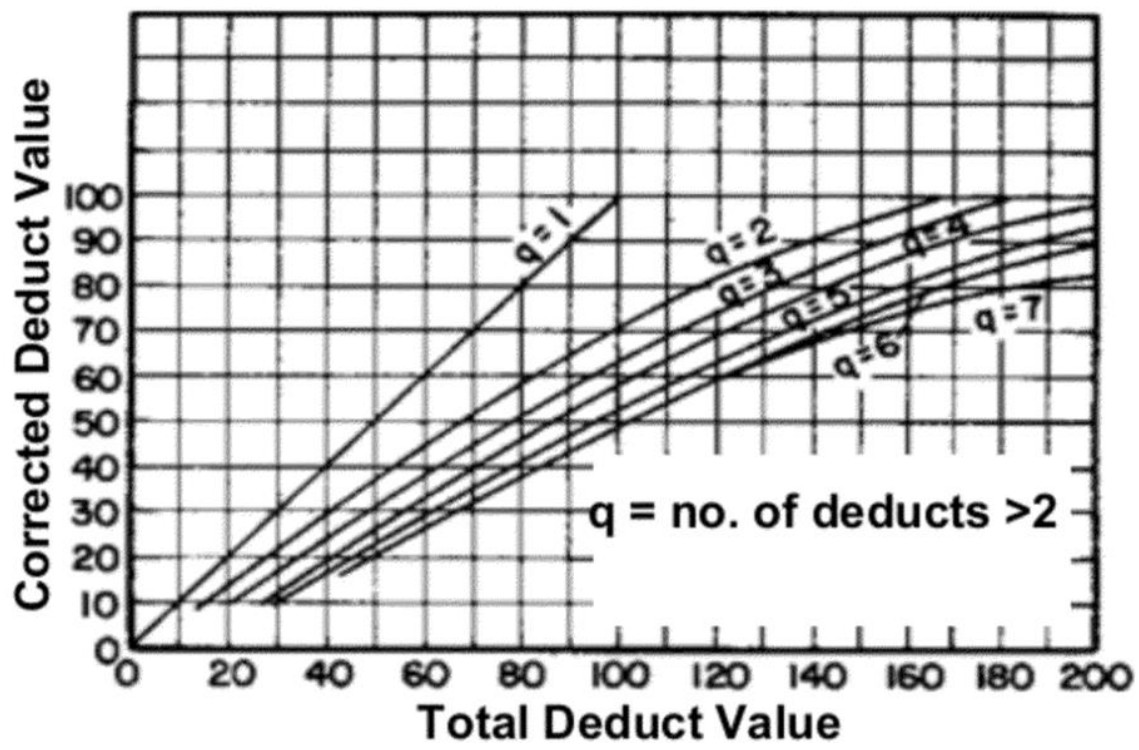

Figure 6: Corrected Deduct Value from Total Deduct Value and number of deducts " $q$ " for HMA (ASTM D6433-11, 2011)

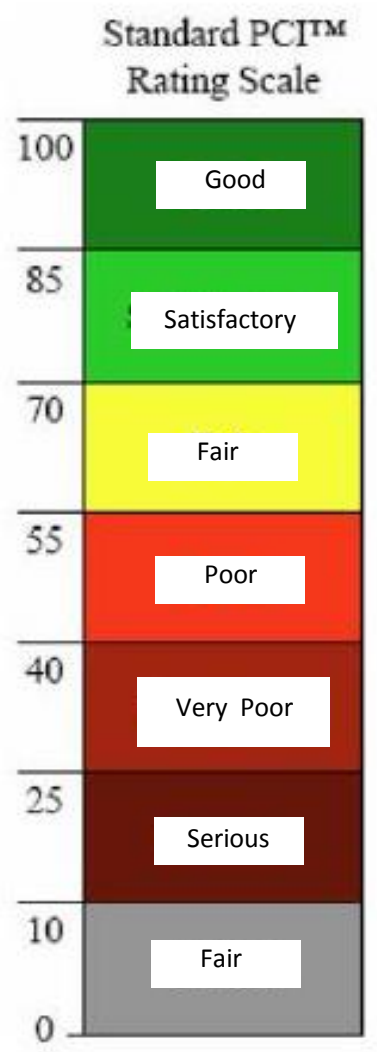

Suggested

Colors

Dark Green

Light Green

Yellow

Light Red

Medium Red

Dark Red

Dark Grey

Figure 7: Standard PCI Rating Scale (after ASTM D6433-11, 2011) 


\subsubsection{MnDOT Surface Rating}

The distress evaluation and analysis system used by the Minnesota Department of Transportation is simpler than the PCI method. MnDOT uses a unique distress identification manual. The distress types used by MnDOT are a subset of the ones used in $\mathrm{PCl}$. However, the severity definitions differ. Table 2 and Table 3 present the distress type, severity and extent measurement for asphalt and concrete pavements respectively. MNDOT considers severity level for three distress types for asphalt pavements and none for concrete pavements. Distress extent is evaluated by the number of occurrences of each severity level of transverse cracking. All other distresses of asphalt pavements are based on the linear feet of the pavement section that have each of the distresses. The percent of the distressed area is determined by dividing the measured distress by the length of the section.

Depending on the pavement type each of the percents are multiplied by the weight factors in Table 4 and Table 5. The resulting products are summed to produce the Total Weighted Percent which is converted to the Surface Rating, SR, using Table 6.

\begin{tabular}{|l|c|c|}
\hline \multicolumn{1}{|c|}{ Distress Type } & Severity Levels & How to Measurement \\
\hline Transverse Cracking & Low. Medium, High & Count \\
\hline Longitudinal Cracking & Low, Medium, High & Lineal Feet \\
\hline Longitudinal Joint Deterioration & Low, Medium, High & Lineal Feet \\
\hline Multiple (block) cracking & None & Lineal Feet \\
\hline Alligator Cracking & None & Lineal Feet \\
\hline Rutting & None & Lineal Feet \\
\hline Raveling \& Weathering & None & Lineal Feet \\
\hline Patching & None & Lineal Feet \\
\hline
\end{tabular}

With the following exceptions, count only the most severe distress in any lineal foot:

- Medium and High severity transverse cracks, raveling/weathering, patching, longitudinal joint cracking and rutting shall be counted in combination with other deficiencies.

- Low severity transverse cracks shall not be counted in the same foot as multiple or alligator cracking.

Table 2: HMA Surface Pavement Distress Types and Measurements (Office of Materials and Road Research Pavement Management Unit, 2003) 


\begin{tabular}{|l|c|c|}
\hline \multicolumn{1}{|c|}{ Distress Type } & Severity Levels & How to Measurement \\
\hline Slight Joint Spalling & None & Number of Joints \\
\hline Severe Joint Spalling & None & Number of Joints \\
\hline Faulted Joints & None & Number of Joints \\
\hline Cracked Panels & None & Number of Panels \\
\hline Broken Panels & None & Number of Panels \\
\hline Faulted Panels & None & Number of Panels \\
\hline Overlaid Panels & None & Number of Panels \\
\hline Patched Panels & None & Number of Panels \\
\hline D-Cracking & None & Number of Panels \\
\hline Slight Longitudinal Joint Spalling & None & Number of Panels \\
\hline Severe Longitudinal Joint Spalling & None & Number of Panels \\
\hline
\end{tabular}

Table 3: Jointed Concrete Pavement Distress Types and Measurements (Office of Materials and Road Research Pavement Management Unit, 2003)

\begin{tabular}{|l|c|c|}
\multicolumn{1}{|c|}{ Distress Type } & Severity & Weighting Factor \\
\hline \multirow{4}{*}{ Transverse Cracking } & Low & 0.01 \\
\cline { 2 - 3 } & Medium & 0.10 \\
\cline { 2 - 3 } & High & 0.20 \\
\hline \multirow{3}{*}{ Longitudinal Cracking } & Low & 0.02 \\
\cline { 2 - 3 } & Medium & 0.03 \\
\cline { 2 - 3 } & High & 0.04 \\
\hline \multirow{2}{*}{ Longitudinal Joint Deterioration } & Low & 0.02 \\
\cline { 2 - 3 } & Medium & 0.03 \\
\cline { 2 - 3 } & High & 0.04 \\
\hline Multiple (block) cracking & - & 0.15 \\
\hline Alligator Cracking & - & 0.35 \\
\hline Rutting & - & 0.15 \\
\hline Raveling \& Weathering & - & 0.02 \\
\hline Patching & - & 0.04 \\
\hline
\end{tabular}

Table 4: HMA Pavement SR Weight Factors (Office of Materials and Road Research Pavement Management Unit, 2003)

\begin{tabular}{|l|c|c|}
\multicolumn{1}{|c|}{ Distress Type } & Severity & Weighting Factor \\
\hline \multirow{2}{*}{ Transverse Joint Spalling } & Low & 0.10 \\
\cline { 2 - 3 } & High & 0.20 \\
\hline \multirow{2}{*}{ Longitudinal Joint Spalling } & Low & 0.10 \\
\cline { 2 - 3 } & High & 0.20 \\
\hline Cracked Panels & - & 0.07 \\
\hline Broken Panels & - & 0.07 \\
\hline Faulted Joints & - & 0.10 \\
\hline Faulted Panels & - & 0.07 \\
\hline $100 \%$ Overlaid Panels & & 0.00 \\
\hline Patched Panels & & 0.14 \\
\hline D-Cracking & & 0.10 \\
\hline
\end{tabular}

Table 5: Jointed Concrete Pavement SR Weight Factors (Office of Materials and Road Research Pavement Management Unit, 2003) 
Table 6 is then used to determine the Surface Rating from the Total Weighted Percent. SR is on a $0-4$ scale where 0 represents the worst pavement possible and 4 represents the best pavement possible.

\begin{tabular}{|c|c|}
\hline Total Weighted Percent & SR \\
\hline 0 & 4.0 \\
\hline 1 & 3.8 \\
\hline 2 & 3.6 \\
\hline 3 & 3.4 \\
\hline 4 & 3.2 \\
\hline 5 & 3.0 \\
\hline 6 & 2.9 \\
\hline 7 & 2.8 \\
\hline 8 & 2.7 \\
\hline 9 & 2.6 \\
\hline 10 & 2.5 \\
\hline 11 & 2.4 \\
\hline 12 & 2.3 \\
\hline 13 & 2.2 \\
\hline 14 & 2.1 \\
\hline 15 & 2.0 \\
\hline $16-17$ & 1.9 \\
\hline 18 & 1.8 \\
\hline $19-20$ & 1.7 \\
\hline 21 & 1.6 \\
\hline $22-23$ & 1.5 \\
\hline 24 & 1.4 \\
\hline $25-26$ & 1.3 \\
\hline 27 & 1.2 \\
\hline $28-29$ & 1.1 \\
\hline $30-33$ & 1.0 \\
\hline $34-40$ & 0.9 \\
\hline $41-47$ & 0.8 \\
\hline $48-54$ & 0.7 \\
\hline $55-61$ & 0.6 \\
\hline $62-68$ & 0.5 \\
\hline $69-75$ & 0.4 \\
\hline $76-82$ & 0.3 \\
\hline $83-89$ & 0.2 \\
\hline $90-96$ & 0.1 \\
\hline $97-100$ & 0.0 \\
\hline
\end{tabular}

Table 6: Total Weighted Percent or Total Weighted Distress Conversion to SR (Office of Materials and Road Research Pavement Management Unit, 2003)

\subsubsection{WVDOH Pavement Surface Rating, PSR}

Pavement Surface Rating, PSR, is an Index developed by Tom Medvick for the West Virginia Division of Highways. This intention was to emulate the simplicity of MnDOT's SR system and the technical accuracy of the PCl's system while also considering the pavement types and distresses most pertinent to West Virginia's highways. The method for evaluation is included in "West Virginia Department of Transportation Division of Highways Special Provision, Section 490: Nine Year Pavement 
Performance Criteria," April 2012. The pavement condition survey and analysis methodology in this special provision represented the WVDOH's initial development of a detailed manual condition survey methodology and served as the foundation of the condition assessment methodologies developed in this research.

Similarly to MnDOT's SR, specific distresses for both concrete and asphalt surfaces are measured and recorded. The distresses, associated severity levels, and associated units of measurement are included in Table 7 and Table 8. The definitions for these distresses and their severity levels are based from the "Distress Identification Manual for the Long-Term Pavement Performance (LTPP) Program," June 2003. The primary differences are that the LTPP manual defines severity levels for raveling/weathering, patching, scaling, and D-cracking, which are not included in the WVDOH Section 490. Section 490 included "Localized Distress" and "Faulted Panels", however, there was no explicit definition of these distresses in either Section 490 or the LTPP manual.

As in MnDOT's SR, each distress-severities combination has an associated weight factor which is shown in Table 9 and Table 10.

\begin{tabular}{|c|c|c|}
\hline \multicolumn{1}{|c}{ Distress Type } & Severity Levels & How to Measure \\
\hline $\begin{array}{c}\text { Transverse } \\
\text { Cracking }\end{array}$ & Low, Medium, High & Count \\
\hline $\begin{array}{c}\text { Longitudinal } \\
\text { Cracking }\end{array}$ & Low, Medium, High & Lineal Feet \\
\hline $\begin{array}{c}\text { Longitudinal Joint } \\
\text { Deterioration }\end{array}$ & Low, Medium, High & Lineal Feet \\
\hline $\begin{array}{c}\text { Block Cracking } \\
\text { Alligator Cracking }\end{array}$ & Low, Medium High & Lineal Feet \\
\hline Rutting & $1 / 4$ " to $1 / 2$ " and greater than $1 / 2 "$ & Lineal Feet \\
\hline $\begin{array}{c}\text { Raveling \& } \\
\text { Weathering }\end{array}$ & None & Lineal Feet \\
\hline Patching & None & Lineal Feet \\
\hline
\end{tabular}

With the following exceptions, count only the most severe distress in any lineal foot:

- Medium and High severity transverse cracks, raveling/weathering, patching, longitudinal joint cracking and rutting shall be counted in combination with other deficiencies.

- Low severity transverse cracks shall not be counted in the same foot as block or alligator cracking.

Table 7: PSR Asphalt Distresses, severity levels, and units (Section 490 Nine Year Pavement Performance Criteria) 


\begin{tabular}{|c|c|c|}
\hline Distress Type & Severity Levels & How to Measure \\
\hline Transverse Joint Spalling & Low, Medium, High & Number of Joints \\
\hline Faulted Joints & Low, Medium, High & Number of Joints \\
\hline Corner Breaks & Low and High & Number of Panels \\
\hline $\begin{array}{c}\text { Faulted Panels (Transverse } \\
\text { Crack) }\end{array}$ & None & Number of Panels \\
\hline Patched Panels & None & Number of Panels \\
\hline D-Cracking & None & Number of Panels \\
\hline Longitudinal Joint Spalling & Low, Medium, High & Number of Panels \\
\hline Localized Distress & None & Number of Panels \\
\hline Transverse Cracks & Low, Medium, High & Number of Panels \\
\hline Longitudinal Cracks & Low, Medium, High & Number of Panels \\
\hline Scaling & None & Lineal Feet \\
\hline
\end{tabular}

Table 8: PSR Concrete Distresses, severity levels, and units (Section 490 Nine Year Pavement Performance Criteria, 2012)

\begin{tabular}{|l|c|c|}
\hline \multicolumn{1}{|c|}{ Distress Type } & Severity & Weight Factor \\
\hline \multirow{3}{*}{ Transverse Cracking } & Low & 0.01 \\
\cline { 2 - 3 } & Medium & 0.10 \\
\cline { 2 - 3 } & High & 0.20 \\
\hline \multirow{3}{*}{ Longitudinal Cracking } & Low & 0.02 \\
\cline { 2 - 3 } & Medium & 0.03 \\
\cline { 2 - 3 } & High & 0.04 \\
\hline \multirow{4}{*}{ Longitudinal Joint Deterioration } & Low & 0.04 \\
\cline { 2 - 3 } & Medium & 0.05 \\
\cline { 2 - 3 } & High & 0.06 \\
\hline \multirow{3}{*}{ Block Cracking } & Low & 0.05 \\
\cline { 2 - 3 } & Medium & 0.10 \\
\cline { 2 - 3 } & High & 0.15 \\
\hline \multirow{3}{*}{ Alligator Cracking } & Low & 0.15 \\
\cline { 2 - 3 } & Medium & 0.25 \\
\cline { 2 - 3 } & High & 0.35 \\
\hline Rutting (greater than $1 / 4$ inch up to $1 / 2$ inch) & & 0.07 \\
\hline Rutting (greater than $1 / 2$ inch) & - & 0.15 \\
\hline Raveling \& Weathering & - & 0.02 \\
\hline Patching & - & 0.04 \\
\hline
\end{tabular}

Table 9: PSR Asphalt Distress Weight Factors (Section 490 Nine Year Pavement Performance Criteria, 2012) 


\begin{tabular}{|c|c|c|}
\hline Distress Type & Severity & Weight Factor \\
\hline \multirow{3}{*}{ Transverse Cracking } & Low & 0.10 \\
\hline & Medium & 0.18 \\
\hline & High & 0.25 \\
\hline \multirow{3}{*}{ Longitudinal Cracking } & Low & 0.10 \\
\hline & Medium & 0.18 \\
\hline & High & 0.25 \\
\hline \multirow{3}{*}{ Transverse Joint Spalling } & Low & 0.06 \\
\hline & Medium & 0.10 \\
\hline & High & 0.22 \\
\hline \multirow{3}{*}{ Longitudinal Joint Spalling } & Low & 0.06 \\
\hline & Medium & 0.10 \\
\hline & High & 0.22 \\
\hline \multirow{3}{*}{ Faulted Joints } & Low & 0.15 \\
\hline & Medium & 0.20 \\
\hline & High & 0.25 \\
\hline \multirow{2}{*}{ Corner Breaks } & Low & 0.04 \\
\hline & High & 0.15 \\
\hline Faulted Panels & - & 0.15 \\
\hline Localized Distress & - & 0.40 \\
\hline D-cracking & - & 0.10 \\
\hline Scaling & - & 0.02 \\
\hline Patched Panels & - & 0.14 \\
\hline
\end{tabular}

Table 10: PSR Concrete Distress Weight Factors (Section 490 Nine Year Pavement Performance Criteria, 2012)

For asphalt surfaces, all distress lengths are totaled for each $1 / 10^{\text {th }}$ mile test section and density percentages are calculated by dividing each total by 528 feet and then multiplying by 100 . This holds true for all distresses with the exception of transverse cracking which is counted by number as opposed to length. In this case, the number of cracks in the section is simply multiplied by two to calculate density (for up to 50 cracks so density percentage does not surpass 100).

For jointed concrete test sections, the amount of slabs within the $1 / 10^{\text {th }}$ mile test section is noted and distress density percentage is calculated based on the amount of affected slabs within the test section. The exceptions here being transverse joint faulting and spalling which are measured in amount of joints affected, and scaling which is measure in linear feet.

The density of each distress is multiplied by the corresponding weight factor and the subsequent weighted distresses are totaled to compute a Total Weighted Distress, TWD. PSR is a function of TWD calculated using Equation 3. 
$P S R=25 *\left[e^{1.386-(0.045 * T W D)}\right]$

PSR is evaluated on a 0-100 scale where 100 is a perfect pavement and PSR decreases as distress increases. Additionally, the special provision includes thresholds for bonus and penalty to contractors based on PSR, bid price, and pavement age. An example of the criteria for bonuses and penalties is shown in Table 11. This example assumes a road paved in 2011. The dates and bonus/penalty amounts vary between contracts.

\begin{tabular}{|c|c|c|c|c|}
\hline Year & $\begin{array}{c}\text { PSR } \\
\text { Criteria } \\
\text { for Bonus }\end{array}$ & Bonus & $\begin{array}{l}\text { PSR Criteria for } \\
\text { corrective action }\end{array}$ & $\begin{array}{l}\text { Penalty for no } \\
\text { corrective action }\end{array}$ \\
\hline $\begin{array}{c}\text { One } \\
\text { Oct. } 31,2012\end{array}$ & 98 & $\$ 10,000$ & $<95$ & $\$ 20,000$ \\
\hline $\begin{array}{c}\text { Two } \\
\text { Oct. 31, } 2013\end{array}$ & 95 & $\$ 10,000$ & $<92$ & $\$ 20,000$ \\
\hline $\begin{array}{c}\text { Three } \\
\text { Oct. 31, } 2014\end{array}$ & 93 & $\$ 25,000$ & $<90$ & $\$ 50,000$ \\
\hline $\begin{array}{c}\text { Four } \\
\text { Oct. 31, 2015 }\end{array}$ & 90 & $\$ 25,000$ & $<85$ & $\$ 50,000$ \\
\hline $\begin{array}{c}\text { Five } \\
\text { Oct. 31, } 2016\end{array}$ & 90 & $\$ 40,000$ & $<85$ & $\$ 80,000$ \\
\hline $\begin{array}{c}\text { Six } \\
\text { Oct. 31, } 2017\end{array}$ & 90 & $\$ 40,000$ & $<85$ & $\$ 80,000$ \\
\hline $\begin{array}{c}\text { Seven } \\
\text { Oct. 31, } 2018\end{array}$ & 85 & $\$ 80,000$ & $<80$ & $\$ 160,000$ \\
\hline $\begin{array}{c}\text { Eight } \\
\text { Oct. 31, } 2019 \\
\end{array}$ & 85 & $\$ 80,000$ & $<80$ & $\$ 160,000$ \\
\hline $\begin{array}{c}\text { Nine } \\
\text { Oct. } 31,2020\end{array}$ & 85 & $\$ 140,000$ & $<80$ & $\$ 280,000$ \\
\hline
\end{tabular}

Table 11: Example of Incentive Performance Criteria for Bonus and Penalty (Section 490 Nine Year Pavement Performance Criteria, 2012)

Several individual distress thresholds for immediate corrective action are also included in the special provision. For example, if any individual rutting measurement in an HMA or any individual transverse joint faulting measurement in a PCC section exceeds $1 / 2$ inch, an immediate investigation will be prompted and corrective action may be required. Lastly, the warranty includes criteria for surface friction and roughness but these have no effect on PSR calculation.

\subsubsection{West Virginia DOH PMS Pavement Distress Evaluation}

The WVDOH uses Deithton Infrastructure Management Systems, dTIMS, software to support its pavement management system, PMS. The pavement condition data that populates the PMS were 
collected with an Automatic Road Analyzer, ARAN, through a contract with Fugro Roadware. The state uses International Roughness Index, IRI, data and pavement surface condition measures for the evaluation of the state's system and the development of the State Transportation Improvement Program, STIP.

The ARAN (shown in Figure 8) "is an advanced pavement data collection vehicle equipped with precision survey systems and software. It is capable of completing any roadway data collection task" (Fugro Roadware Inc., 2012).

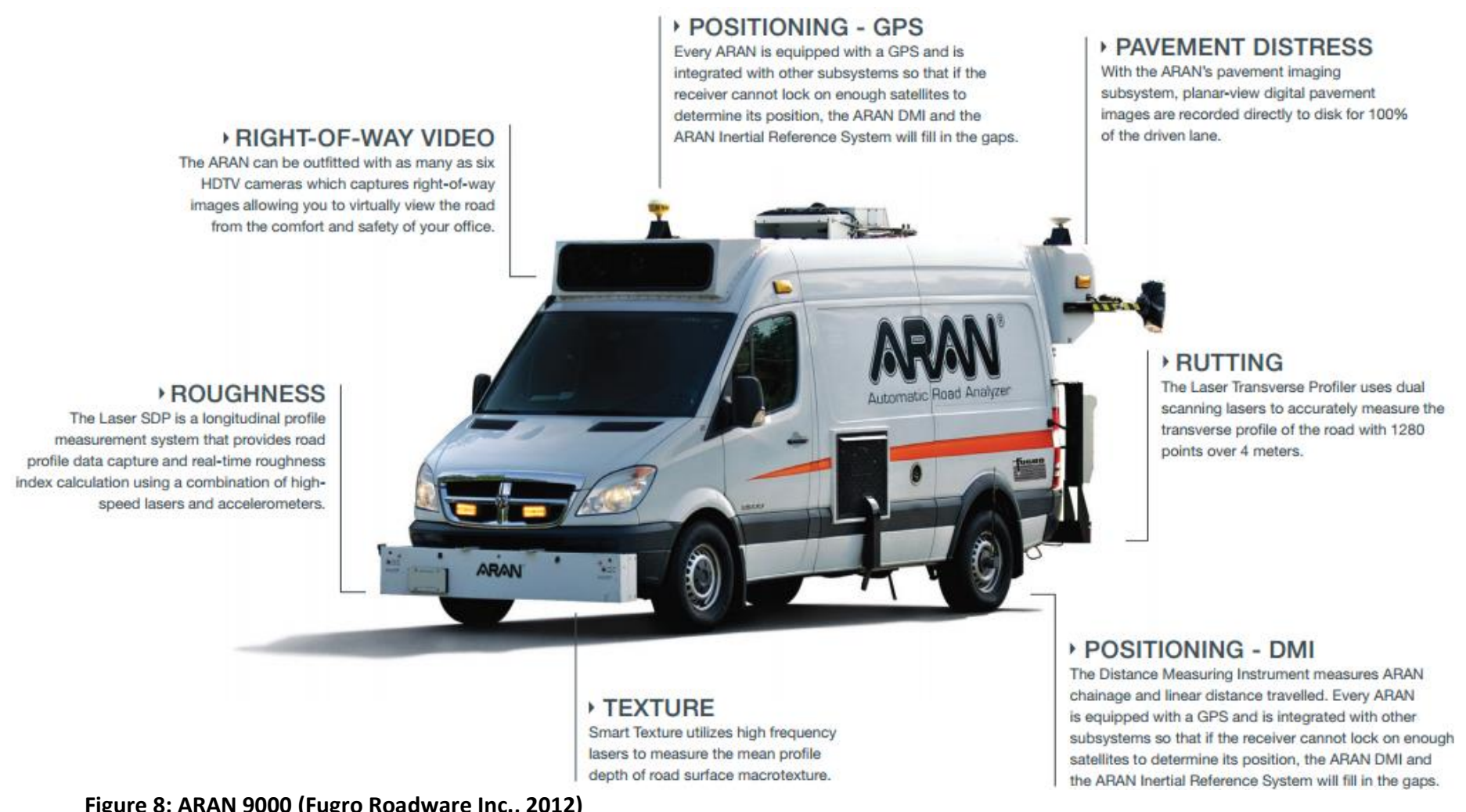

IRI and the pavement surface condition measures, specifically requested by the WVDOH from this vehicle, are used to calculate Composite Condition Index which is then used to make the most efficient pavement rehabilitation decisions for West Virginia.

Composite Condition Index, $\mathrm{CCl}$, is a pavement condition indicator for rigid or flexible pavements developed by Naveed Sami in 1997 for the WVDOH. CCI is a 0-5 scale where 5 represents perfect pavement and 2.5 is the trigger point for investigation and appropriate treatment. $\mathrm{CCl}$ is a function of multiple other indices that represent roughness and different distresses of the pavement. 
All distress types used in the indices are included in the LTPP distress identification manual and the specific distress definitions "generally follow LTPP definitions" (Sami, 1997). All distresses are evaluated as low, medium, and high severity, however the thresholds for the severity levels are slightly different from the values specified in the LTPP manual.

Currently the WVDOH contracts with Fugro Roadware Inc. to survey pavements with the ARAN and reduce the ARAN information to distress measurements using a combination of automated distress identification programs and human reviewing of the imaging.

For flexible pavements, $\mathrm{CCl}$ is a function of Rut Depth Index, RDI, Structural Cracking Index, SCI, Environmental Cracking Index, ECI, and Present Serviceability Index, PSI. PSI is calculated using Equation 4 which is a function of IRI. RDI is calculated using Equation 5 which is a function of the average Rut Depth in inches of the test section. $\mathrm{SCl}$ is calculated using Equation 6 which is a function of the extent (percent of area affected) of low, medium, and high severity Alligator Cracking (AL, AM, AH respectively) and low, medium, and high severity Longitudinal Cracking (LL, LM, LH respectively). ECI is calculated using Equation 7 which is a function of the extent (percent of area affected) of low, medium, and high severity Transverse Cracking (TL, TM, TH respectively) and low, medium, and high severity Block Cracking ( $\mathrm{BL}, \mathrm{BM}, \mathrm{BH}$ respectively). $\mathrm{CCl}$ simply equals the lowest value of $\mathrm{PSI}, \mathrm{RDI}, \mathrm{SCl}$, and $\mathrm{ECI}$ for a given test section.

$P S I=5 * e^{-0.0041 * I R I}$

$$
R D I=5-6.65 * R D^{1.41}
$$

$S C I=5-0.21 * A L^{0.67}-0.24 * A M^{0.69}-0.40 * A H^{0.80}-0.28 * L L^{0.73}-0.32 * L M^{0.75}-0.60 * L H^{0.89}$

$E C I=5-0.32 * T L^{0.75}-0.40 * T M^{0.80}-0.60 * T H^{0.89}-0.24 * B L^{0.69}-0.28 * B M^{0.73}-0.40 * B H^{0.80}$

For rigid pavements, $\mathrm{CCl}$ is a function of Joint Condition Index, $\mathrm{JCl}$, and Concrete Slab Index, CSI, and Present Serviceability Index, PSI. PSI is measured using Equation 4 as it is in the case of asphalt pavements. $\mathrm{JCl}$ is calculated using Equation 8 which is a function of the extent (percent of slabs affected) of low, medium, and high severity Faulting (FL, FM, FH respectively) and Distressed Joints, DJ. 
In this case Distressed Joints are joints with faulting at any severity level. CSI is calculated using Equation 9 which is a function of the extent (percent of slabs affected) of low, medium, and high severity Transverse Cracking (TL, TM, TH respectively) and low, medium, and high severity Transverse \& Longitudinal Cracking (SL, SM, SH respectively). Transverse \& Longitudinal Cracking represents any slabs affected by either transverse or longitudinal cracks (or both). CCl simply equals the lowest value of PSI, $\mathrm{JCl}$, and CSI for a given test section.

$J C I=5-0.13 * F L^{0.56}-0.16 * F M^{0.60}-0.17 * F H^{0.62}-0.13 * D J^{0.56}$

$C S I=5-0.13 * T L^{0.75}-0.17 * T M^{0.80}-0.20 * T H^{0.89}-0.20 * S L^{0.69}-0.28 * S M^{0.73}-0.40 * S H^{0.80}$

\subsection{Deflection Testing and Analysis of Deflection Data}

Falling Weight Deflectometer, FWD, data were collected twice during the evaluation period. Typically these data are used for estimating the modulus of elasticity of pavement layers (Gucunski et al., 2009). The WVDOH does not have standard practice for analyzing FWD data. The following describes three backcalculation methodologies for analyzing FWD data.

\subsubsection{Falling Weight Deflectometer and Backcalculation of Layer Moduli}

The Falling Weight Deflectometer, FWD, is an instrument that simulates the deflection of pavement caused by a truck driving over its surface. It is the most commonly accepted form of nondestructive testing for evaluating structural condition of pavements. Throughout the country, the majority of FWD's owned by state highway agencies are manufactured by DYNATEST (Alavi et al., 2008).

The FWD applies an impulse load generated by dropping a mass from a specified height onto a circular load plate with a diameter of $300 \mathrm{~mm}$. The DYNATEST Model 8000 FWD has the ability to drop a weight ranging from 110 to $770 \mathrm{lbs}$ from a height of 0.8 to 15 inches to generate a peak force ranging from 1500 to $27,000 \mathrm{lbs}$. Although the mass which is dropped can be changed, it is recommended that the load applied to the pavement be adjusted by changing the height of the drop (Gucunski et al., 2009). The LTPP protocol specifies four drops at four load levels at each test location on flexible pavements (Schmalzer, 2006). The target load levels and acceptable ranges are:

- $\quad 6.0$ kips (5.4 to 6.6)

- $\quad 9.0$ kips (8.1 to 9.9) 
- $\quad 12.0$ kips (10.8 to 13.2$)$

- $\quad 16.0$ kips (14.4 to 17.6$)$

According to the LTPP Manual for Falling Weight Deflectometer Measurements, FWD testing on rigid pavements does not require the 6.0 kip load level.

An array of sensors (also called geophones) measures maximum deflections in mils (1/1000 inches) at certain distances from the plate's center. The entire instrument, including load plate, weights, and geophones, is usually contained within a trailer and pulled by a truck or van. The mass being dropped must be selected before testing and cannot be changed during testing. Otherwise, the procedure is operated completely from a laptop within the vehicle. Figure 9 shows an example of a DYNATEST FWD and the van towing it.
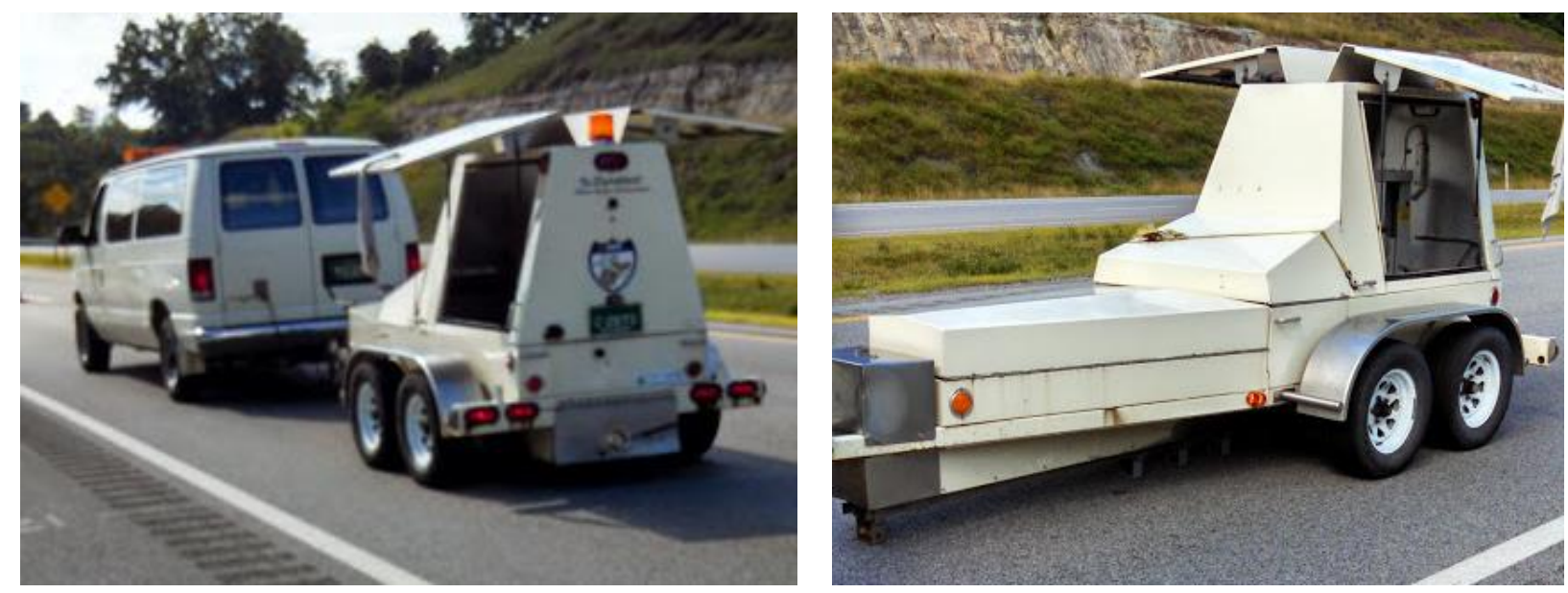

Figure 9: WVDOH DYNATEST Falling Weight Deflectometer Trailer on US 35

The LTPP Manual for Falling Weight Deflectometer Measurements outlines several test plans for various pavement types and the experiment designations of FWD tests. Testing structural properties of pavement materials is included within the General Pavement Studies test plan. For flexible pavements, the LTPP requires tests performed every 25 feet mid-lane and in the outer wheel path within a test section. For rigid pavements, the LTPP requires test performed in the following locations on 20 continuous slabs:

- Mid-Slab (transversely), Mid Panel (longitudinally)

- Slab outer edge, Mid-Panel (longitudinally) 
- Slab corner

In all cases, the sensors radiate from the center of the load plate parallel to the pavement centerline. The sensor spacing however, can be configured by the user. The DYNATEST Model 8000 FWD can accommodate sensors up to 8 feet (96 inches) from the center of the loading plate. Typical sensor arrangements typically include 7 or 9 sensors with spacing relative to the center of the load, of:

- $-12,0,8,12,18,24,36,48$, and 60 inches

- $0,8,12,18,24,36$, and 60 inches

- $-12,0,12,18,24,36$, and 60 inches

- $0,12,24,36,48,60$, and 72 inches

Typically, sensors in the negative direction are only used for load transfer across joints of jointed concrete pavements and across cracks in pavement to check crack propagation. (Gucunski et al., 2009) The LTPP requires that all nine sensor FWDs use the first spacing in the previous list. Seven sensor FWDs are required to use the second spacing on flexible pavements and the third spacing is for rigid pavements. Many other sensor spacing combinations have been suggested which sometimes include a sensor at 72 inches. In any case, a deflection basin is formed that should have a maximum deflection at the sensor centered under the load ( 0 inches) and deflections will decrease as the distance from the load plate increases. An exaggerated basin example is shown in Figure 10.

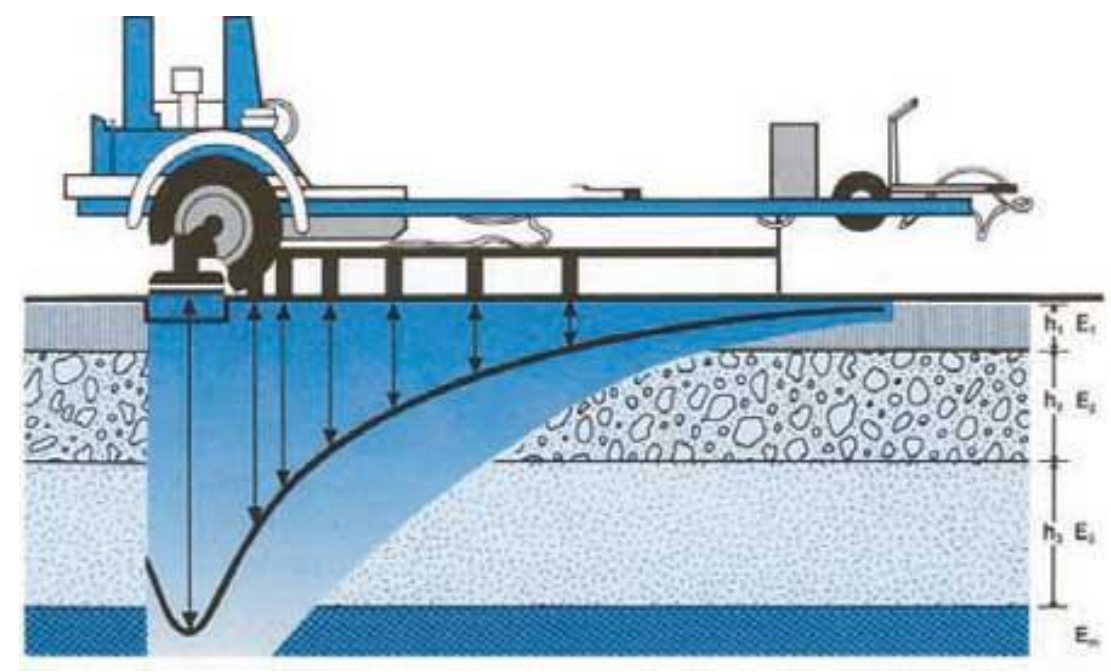

Figure 10: Falling Weight Deflectometer Deflection Basin (Cornell University, 2005) 
From these measured deflections, stiffness related physical properties of the pavement can be estimated. "One of the more common analysis methods of the deflection data is to backcalculate material response properties for each structural layer within the pavement structure. The specific layer property determined from the peak deflection basin is Young's modulus, determined by an elastic layer based backcalculation program" (Killingsworth and Von Quintus, 1997). "For rehabilitation design processes, the Mechanistic-Empirical Pavement Design Guide, MEPDG, relies heavily on the material stiffness back calculated from the deflection measurements taken from existing pavements" (Gucunski et al., 2009). Many linear elastic layer theory programs exist that will solve for a deflection basin using known layer thicknesses and applied loads while iteratively varying moduli inputs until the error between the predicted and measured deflections is a minimum. Although this process is widely used, there are many problems that can arise. Upon testing several backcalculation programs for this purpose, Killingsworth and Von Quintus (1997) concluded that, "There is no unique solution for a particular deflection basin; engineering judgment must be used in determining reasonable solutions. Most programs based on elastic layer theory can be used to find a combination of layer moduli with an error term less than 2.0 percent per sensor. However, these layer moduli can be consistently and significantly different between these programs, which prevent the combination and random use of different programs." In order to better understand these inevitable errors the LTPP Manual for FWD Measurements additionally requires notes be taken by field engineers regarding pavement surface distresses at test locations. "There are limitations to accurate prediction of the layers' moduli in the areas with longitudinal or transverse cracking, since these cracks disturb the assumption of the layers extending to infinity" (Norouzi, 2013).

For flexible pavements, the estimated modulus values are dependent on the pavement temperature. For instance asphalt moduli can range from over 5,000 ksi at low temperatures to below $100 \mathrm{ksi}$ at high temperatures. Additionally, temperature gradients will occur within pavements as air temperature and sun exposure change. A study on air temperature and resulting in situ pavement temperatures was conducted by Alnouri (1972). The averaged results (independent of solar radiation) are summarized in Figure 11. The left portion of Figure 11 shows daily high air temperature and the maximum average temperature of the pavement through its depth. The right portion of Figure 11 shows when the maximum temperature could be expected at select depths relative to an assumed air temperature daily fluctuation. 

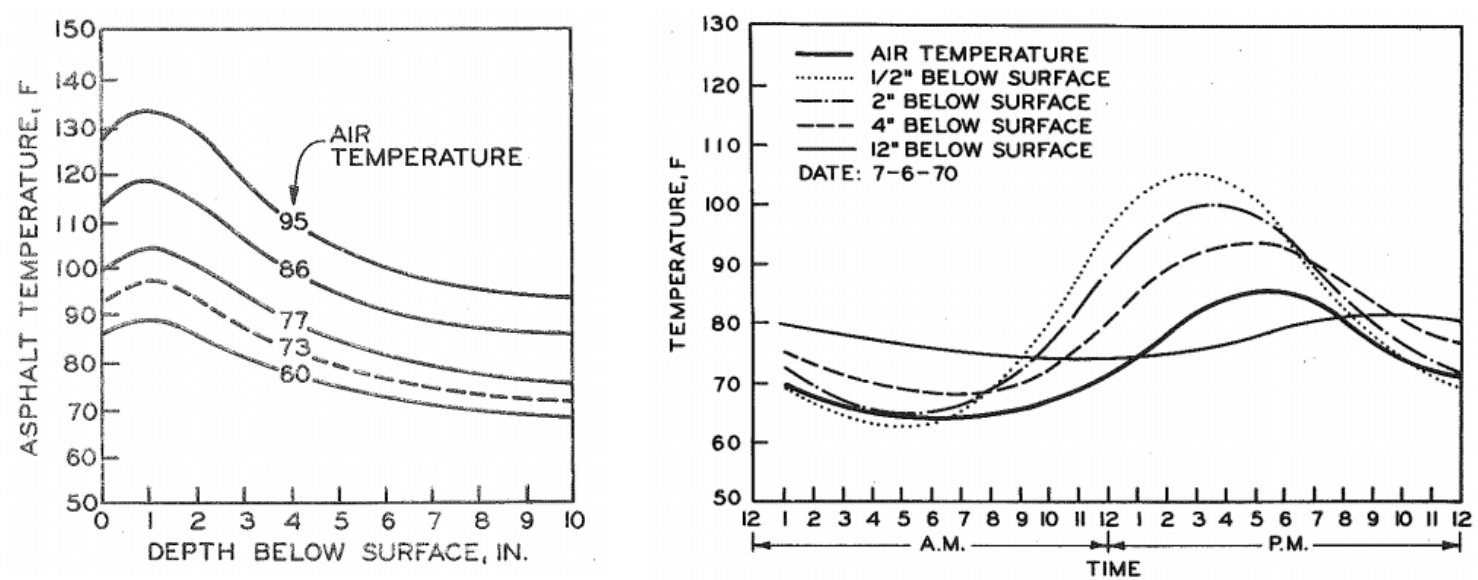

Figure 11: Averaged graphical representation of temperature gradient within asphalt pavements (Alnouri, 1972)

Pavement depth, aggregate, percent binder, air voids, etc, will affect the actual temperature of a pavement. The LTPP requires drilling and probing pavements at 5 depths to determine subsurface temperatures when obtaining FWD data. Most backcalculation programs have inputs for temperature when analyzing flexible pavements so a temperature correction can take place. A common temperature gradient prediction equation is the LTPP BELLS2 model originally presented by Lukanen et al. (2000). The model, as shown in Equation 10, requires inputs of a surface temperature, time of day, average air temperature from the previous day, and a distance below the surface for temperature to be calculated.

$T_{d}=2.78+0.912 * I R+\{\log (d)-1.25\} *\{-0.428 * I R+0.553 *(1-$ day $)+2.63 *$

$\left.\sin \left(h r_{18}-15.5\right)\right\}+0.027 * I R * \sin \left(h r_{18-13.5}\right)$

Where:

$\mathrm{T}_{\mathrm{d}}=$ Pavement temperature at depth $\mathrm{d},\left({ }^{\circ} \mathrm{C}\right)$

$\mathrm{IR}=$ Pavement surface temperature, $\left({ }^{\circ} \mathrm{C}\right)$

$\mathrm{d}=$ depth at which material temperature is to be predicted, $(\mathrm{mm})$

1-day = Average air temperature the day before testing, $\left({ }^{\circ} \mathrm{C}\right)$

$\mathrm{hr}_{18}=$ Time of day in a 24-hr clock system, but calculated using an 18-hr asphalt concrete temperature rise and fall time cycle where all times from 0500 to 1100 should be entered as either 5:00 AM or 11:00 AM and minutes should be converted to decimal form

Regardless of the program selected, the result of the backcalculation process will be moduli values which quantify stiffness for layers defined in the pavement structure. For asphalt or concrete layers this is modulus of elasticity. For unbound materials such as free draining base or subgrade soil, 
the resilient modulus is determined which is an estimate of modulus of elasticity independent of confining pressures, and moisture related properties which often cannot be known. The estimated resilient modulus of the subgrade soil is directly used in the determination of a pavement's current structural condition and remaining life.

A pavement's current structural condition can be quantified by effective structural number, $\mathrm{SN}_{\text {eff, }}$ for flexible pavements and effective slab thickness, D, for rigid pavements. A process for using FWD results to determine both is described in AASHTO 93 Pavement Design Guide. Appendix L of the design guide contains a procedure specifically for using FWD results to calculate effective structural number. "The $\mathrm{SN}_{\text {eff }}$ determination uses a model based on stiffness theory and a two-layer pavement structure. The stiffness of the constructed material, regardless of its constituent components, is represented by the term $E_{p}$, which is an average elastic modulus for all the material above the subgrade" (Pologruto, 2007). This process is summarized in Equation 11 and 12. In Equation 12, $M_{R}$ of the subgrade must be independently determined by backcalculation before $E_{p}$ may be solved from the remainder of the FWD outputs.

$S N_{\text {eff }}=0.0045 D \sqrt[3]{E_{p}}$

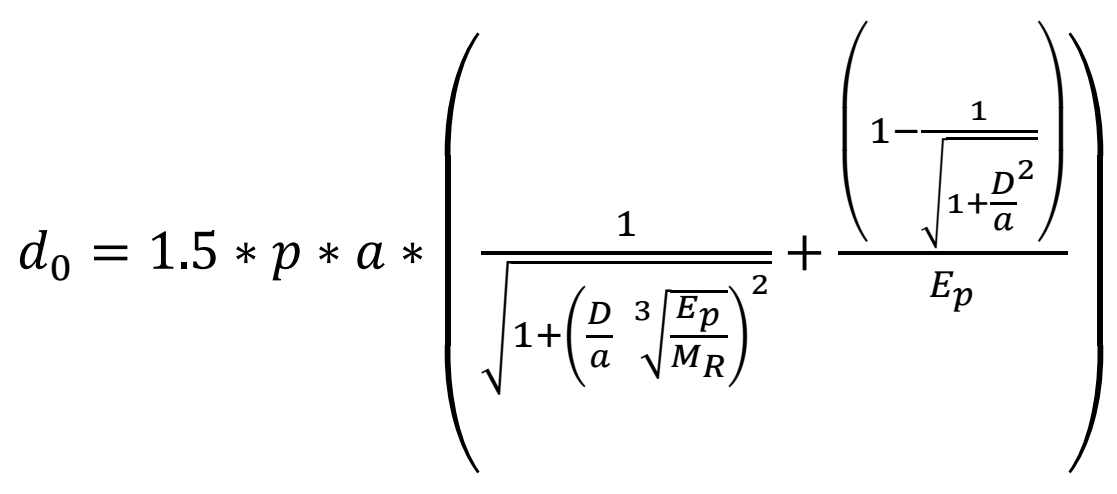

Where:

$\mathrm{SN}_{\text {eff }}=$ effective structural number

$D=$ total thickness of pavement structure resting on subgrade (in)

$E_{p}=$ effective modulus for total pavement structure (lbf/in2)

$\mathrm{d}_{0}=$ centerline deflection measured by the FWD (in)

$\mathrm{p}=$ loading plate pressure applied by the FWD (Ibf/in2)

$\mathrm{a}=$ loading plate radius (in)

$M_{R}=$ subgrade resilient modulus (lbf/in2) 
Interestingly, a vast amount of research suggests subgrade resilient modulus as determined by FWD backcalculation will differ from the modulus of the same soil sample determined through triaxial testing in a lab. Based on experimental observation, the AASHTO 93 pavement design guide recommends a correction factor up to 0.33 and 0.25 for subgrade resilient modulus below flexible and rigid pavements respectively to obtain modulus values suitable for design. A 1999 report which mechanistically analyzed FWD data using a finite element method approach concluded that "the correction factors reached in [the] study agree closely with the AASHTO experience-based correction factors" (Shoukry and William, 1999). In a conversation with Trenton Clark (Clark, 2014), Director of Engineering at Virginia Asphalt Association, it was confirmed that many state highway agencies employ policies based on this knowledge. Virginia Department of Transportation uses a "rule of thumb" that backcalculated subgrade resilient moduli should be divided by 3 before being recorded as the "analysis" modulus of the soil. Additionally for VDOT, if that value after being divided by 3 is still over 10,000 psi, it should be recorded as 10,000 psi.

\subsubsection{MODULUS 6.1}

MODULUS was developed in the early 1990's by the Texas Transportation Institute, TTI to process FWD data for the Texas Department of Transportation, TxDOT. In 1993, a study was performed by the Strategic Highway Research Program, SHRP, which reviewed 17 moduli backcalculation programs and compared the three best. The study concluded that MODULUS was superior to the rest in terms of repeatability, reasonableness of results, deflection matching errors, ability to match assumed moduli from simulated deflection basins, and versatility (PCS/Law Engineering, 1993). A 1999 study which compared several backcalculation programs concluded that MODULUS 5.0 rendered the most consistent moduli outputs and was the most user friendly when compared to its contemporaries (William, 1999).

MODULUS 6.1 was updated in September 2013. However, the most recent user manual available is specifically for its predecessor MODULUS 6. Most features and interfaces remained unchanged.

Since its development, MODULUS has used the multi-layer elastic theory forward calculation subroutine known as WESLEA. WESLEA, like all multi-layer elastic theory programs, is based on Burmister's original theory which assumes that all materials within the pavement structure are homogeneous, isotropic, and linearly elastic. This subroutine is used to develop a database of potential 
deflection bases for a given pavement structure due to a specified load (William and Shoukry, 1999). "A pattern search technique is then used to determine the set of layer moduli that best fits the measured basin." (PCS/Law Engineering, 1993) MODULUS's pattern search algorithm and interpolation scheme makes it much faster than other iterative forward calculating programs (Lytton and Germann, 1989). "Because the program does not use a forward calculation scheme in the iterative process, it is particularly suited for the analysis of large numbers of deflection basins measured on pavement with the same structure." (PCS/Law Engineering, 1993) Like any other moduli backcalculation technique, the program cannot guarantee that given deflection basins are generated by unique parameters. Engineering judgment entering seed moduli and ranges for layers is required to keep error to a minimum.

MODULUS 6.1 can import falling weight deflectometer data files in Dynatest Edition 9, 10, and 20 (.FWD, .R80, or .R32) formats. This version of the program is specifically for analyzing asphalt pavements. The program will automatically make adjustments to the seed moduli range of asphalt layers depending on the asphalt temperature input. All layers defined require inputs Poission's ratio as well as maximum and minimum moduli values except for subgrade modulus which only requires a most probable value. The program also provides a guide for suggested inputs based on layer material and condition. The user has the option to select a drop within the FWD drop sequence and analysis is performed on that drop for all stations within the FWD file. The program screens deflection data for drops with non-decreasing deflections or otherwise irregular deflection basins and give the user the option to exclude those drops. MODULUS will output backcalculated moduli values for all defined layers, a depth to bedrock and provide an absolute error per sensor as a measure of confidence. A remaining life analysis may also be performed which will output a pavements expected remaining life in years and each layers current condition on an ordinal scale if traffic data were input.

\subsubsection{MODTAG}

MODTAG was developed cooperatively by Cornell University and the Virginia Department of Transportation. It is a combination of programs otherwise known as TAG and MODCOMP. TAG (Test and Guess) is based on AASHTO 1993 equations and was developed by the Virginia Department of Transportation, VDOT. MODCOMP (Modulus Computation), which was developed by Cornell University, is a multi-layer elastic analysis program. MODTAG requires inputs of layer thicknesses, Poisson's ratios, and seed moduli (as opposed to acceptable moduli ranges) for all layers. Within MODTAG, MODCOMP uses the forward moduli calculating subroutine CHEVLAY to compute surface deflections based on the 
inputs. The program iteratively changes the seed moduli until a deflection basin is established that resembles the observed basin within a given tolerance.

The 1999 study by William comparing MODCOMP3, MODULUS 5.0, and EVERCALC 4.0, concluded that MODCOMP3 "resulted in moduli values which seemed to be reasonable and within the material range. However, the resulting modulus value of the concrete layer in the rigid pavement section seemed to be high compared with those resulting from the other two programs." The study also stated that MODCOMP3 was "the most user-sensitive and required more engineering judgment."

According to the MODTAG user manual, "The main objective of MODTAG is to provide the engineer the ability to analyze raw deflection data and to use the results in the pavement rehabilitation design process." The MODTAG user manual also suggests using several methods when analyzing raw FWD data and comparing to one another before any decisions are made. Additionally this information should be used in combination with visual inspection, laboratory testing, and engineering judgment to be most useful.

The program can import falling weight deflectometer data files in Dynatest Edition 20 (.FWD or .R80), Dynatest Edition 25 (.F25), or AASHTO PDDX file formats. Unlike some other FWD analysis programs, MODTAG can only analyze files that represent test sections with identical weight drop sequences at each station. If for any reason a drop load level or a single drop were excluded from any station, MODTAG cannot analyze that file. Additionally outputs are recorded as averages of all drops within a load level at a certain station.

Once a file is imported, number of load levels and drops per load level are input by the user. MODTAG has the ability to perform backcalculation on rigid or flexible pavements provided the user defines any layers as such. A single seed moduli value is required for all layers. MODTAG uses the BELLS3 temperature correction model which uses FWD temperature data and a user input for the average air temperature for the previous day. The user also has the option to directly input the pavement temperature instead. Within the flexible pavement analysis, the program has the ability to output estimated subgrade modulus, effective structural number, and ESAL's until failure.

\subsubsection{BAKFAA}

BAKFAA is a layer moduli backcalculation program created by the Federal Aviation Administration. This program is available as a free download on several websites including faa.gov. This program uses the LEAF linear elastic analysis program. Layers are defined and seed moduli are 
iteratively changed until a calculated deflection basin matches the observed one within a tolerance. This program is far simpler than the previous two mentioned as all user inputs are included in one window. A single deflection basin can be automatically or manually input along with the layer properties: seed modulus, Poisson's Ratio, and thickness. Unlike the other programs, once an observed deflection basin and pavement structure is input and the program is initiated. The user can watch the moduli values change as the iterations are tallied. Additionally, a plot of the calculated deflection basin is shown as it converges toward the observed basin.

\subsection{Summary of Literature}

PSR as defined in the April 2012 version the WVDOH Special Provision Section 490 was used as a foundation for evaluation and comparison of the condition of the pavements within US 35 . With a comprehensive understanding of this method and the history of its development, improvements to the accuracy and repeatability of the PSR calculation process will be made if necessary. A comparison to PCI will be the most relevant and informative analysis of PSR.

While many backcalculation programs are available in the literature, three programs that have been found by previous researchers to provide the "most reasonable" results were reviewed. Since the literature does not support the selection of one of these programs, all three were applied during this research. 


\section{Chapter 3. Methodology}

This chapter describes the initial process used for the collection and analysis of the pavement distress data for both the manual condition survey and the automated survey performed for the pavement management system. This is followed by a description of the methodology for analyzing the FWD data.

\subsection{Pavement Surface Rating Data Collection and Analysis Process}

The following section is separated into two parts. The first covers the 2012 Special Provision, Section 490 method used for pavement surface distress data collection. The second covers the process that was used to calculate Pavement Surface Rating. The purpose of these sections is mainly to establish a starting point for revisions made to the process.

\subsubsection{Data Collection Process for Pavement Surface Rating}

For each $1 / 10^{\text {th }}$ mile test section a folder exists containing the distress survey diagrams for the test section. Each sheet contains two grid delineated segments representing a fast and slow lane. For asphalt sections, each diagram contains 50 feet of length per lane. Concrete section diagrams contain 60 feet (approximately 4 slab lengths) per lane. Figure 12 and Figure 13 are examples of blank diagram sheets for asphalt and concrete sections. Each diagram sheet was marked with a start and end station to identify the sequence in the sections.

Surface distress surveys were conducted by a mixed group of WVDOH employees and West Virginia University graduate students. Groups of 2 to 4 people walked the length of each segment with a measuring wheel and recorded observable surface distresses on the diagrams to scale and in the location observed. The WVDOH provided traffic control to close the slow lane during the data collection. The distresses recorded for asphalt sections were:

- Transverse Cracking

- Longitudinal Cracking

- Longitudinal Joint Deterioration

- Block Cracking

- Alligator Cracking

- Rutting

- Raveling and Weathering

- Patching 
The distresses recorded for concrete sections were:

- Transverse Joint Spalling

- Faulted Joints

- Corner Breaks

- Faulted Panels

- Patched Panels

- D-Cracking

- Longitudinal Joint Spalling

- Localized Distress

- Transverse Cracks

- Longitudinal Cracks

- Scaling

For rutting measurements, a five foot straight edge was placed over the wheel path and a maximum distance between the bottom of the straight edge and the road surface was recorded. This was done in five locations throughout each asphalt section and in both directions at approximately foot marker 64, 164, 264, 364, and 464. All other distresses were identifiable and observable with by eye. Most of these distresses include severity thresholds which require the surveyors to additionally identify specific features of the distress. Rulers were used to determine the width of a crack or any other small measurement. Definitions for each distress and severity are contained within the April 19, 2012 version of "West Virginia Department of Transportation Division of Highways Special Provision, Section 490: Nine Year Pavement Performance Criteria." In many cases this document defers definition to the "Distress Identification Manual for the Long Term Pavement Performance Program".

Distress diagram forms include examples for the method of recording some normal distresses. Adherence to these symbols was to be maintained and in every case the symbols should be accompanied by a label for the observed severity when applicable.

The same diagrams were used for each survey date but a different colored pen or pencil was used to for the different survey dates. This allowed a way for distress progression to be tracked. If unusual discrepancies between surveys occurred, such as the disappearance of a previously noted distress, notes would be made, but under no circumstance were previous surveys altered. The analysis of the data was performed under the assumption that all distress data were accurate at the time of observation. 


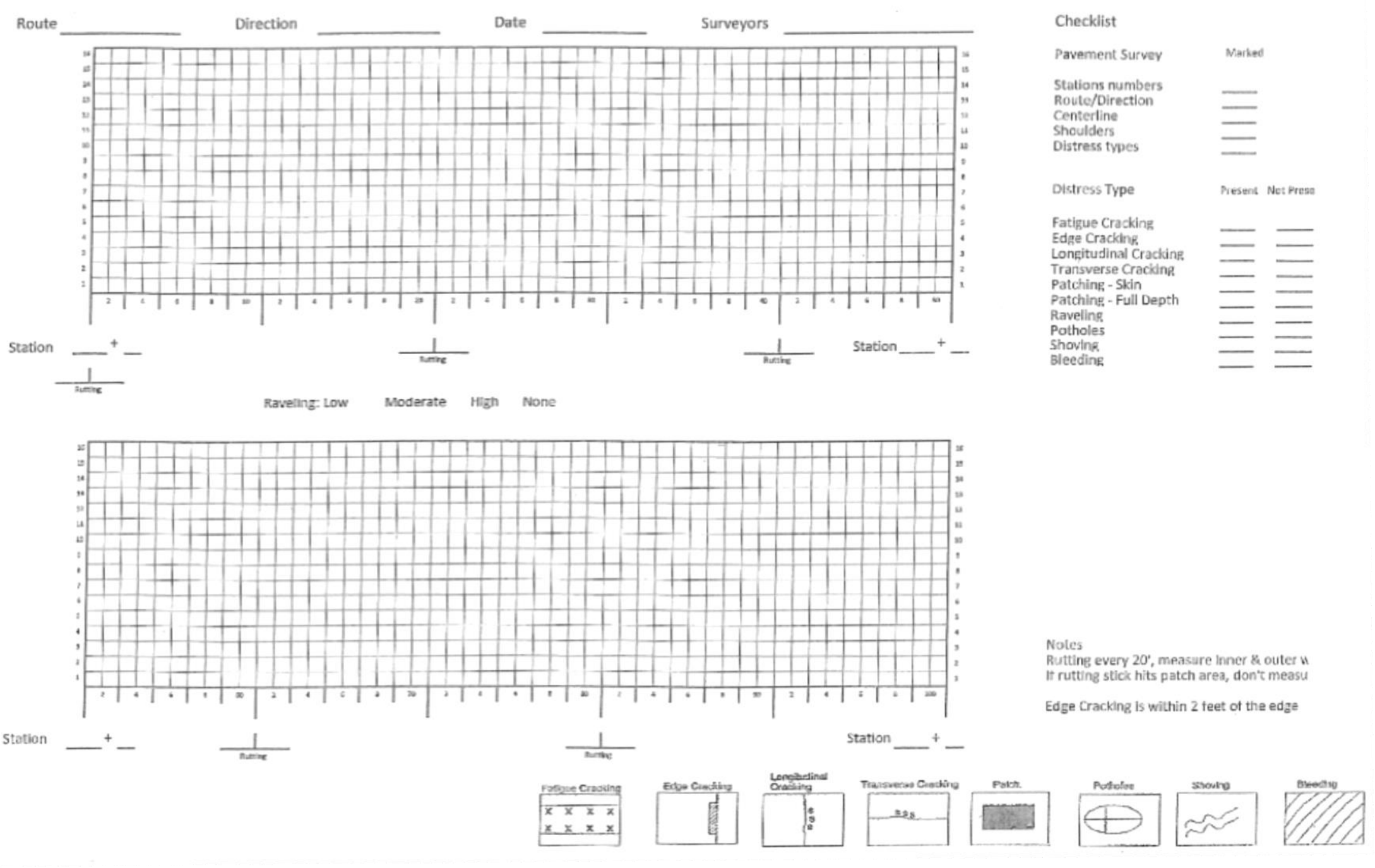

Figure 12: Asphalt Section Surface Distress Survey Diagram Segment 


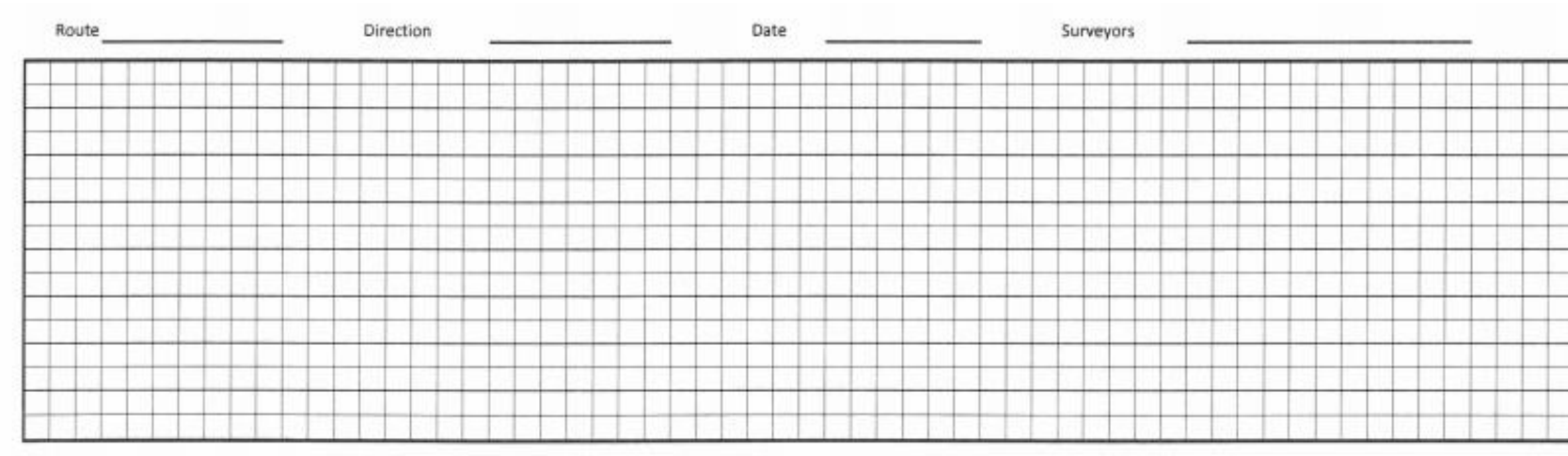

Station

Straion _-

Checklist

Marked

Stations numbers

Route/Direction

houlders

二

Distress types

ongitudinal Cracking

Transverse Joint Crackin

ongitudinal Joint Spalling

Faulted Joint

Corner Breaks

Faulted Panels

Localized Distres

p-cracking

Patched Panels
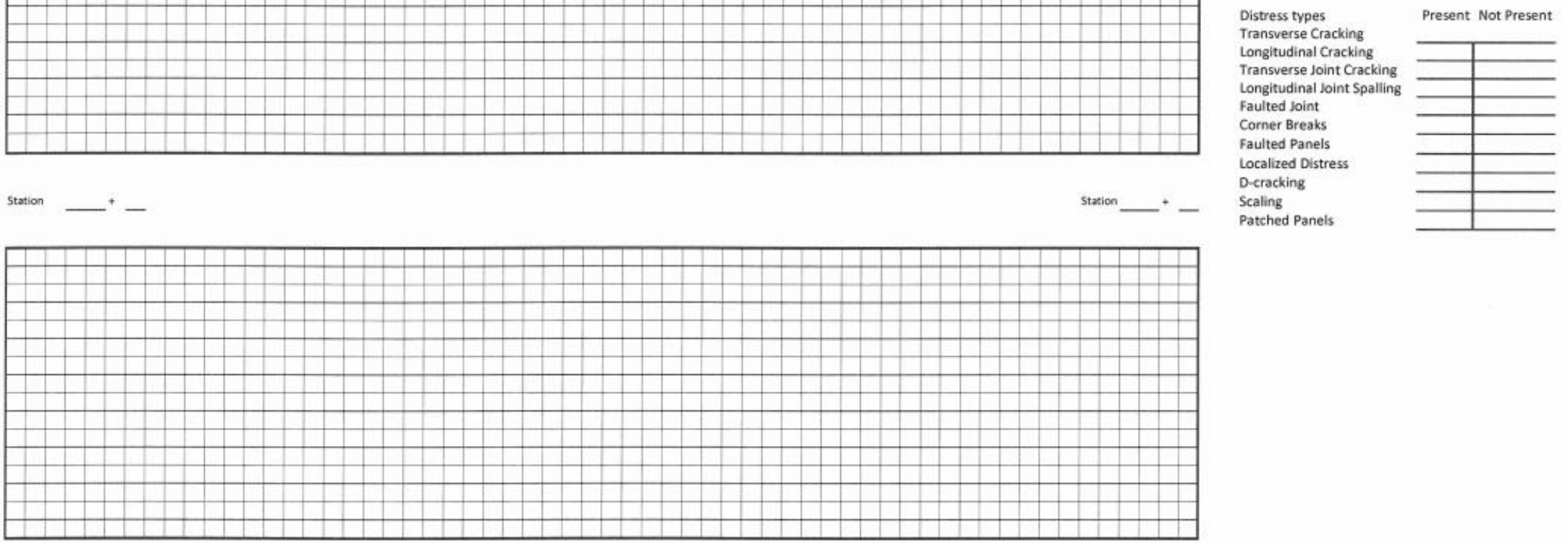

Station

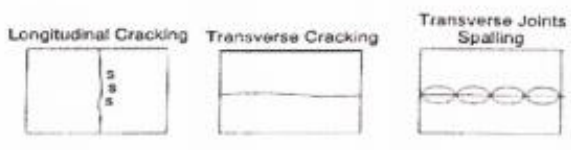

Strtion ${ }_{-}^{+}-$

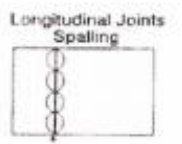

Fauting Jonts

V V

\section{Figure 13: Concrete Section Surface Distress Survey Diagram Segment}




\subsubsection{Data Analysis Process for Pavement Surface Rating}

The first analysis of surveyed surface distress data occurred in July of 2013. At this point three surveys had been completed. US 35 was surveyed during May 2012 (Survey 1), October 2012 (Survey 2), and April 2013 (Survey 3). PSR was to be initially calculated as defined in the April 19, 2012 version of "West Virginia Department of Transportation Division of Highways Special Provision, Section 490: Nine Year Pavement Performance Criteria." This process is described in detail within the WVDOH Section of Surface Distress Ratings in the Literature Review chapter.

Over the course of the research, an Excel file was used to record the observed distresses and calculate PSR. This file includes spreadsheets for pavement type and direction (northbound asphalt, southbound asphalt, northbound concrete, and southbound concrete sections). Each of these spreadsheets consists of a matrix of individual tables which correspond to relevant distress types labeled across the top and the applicable test sections labeled on the left. Thus there is a table which corresponds to every combination of test section and distress type relevant to the calculation of PSR within US 35. Each distress type is divided into columns first by severity level as specified for each distress. The severities are then divided by survey number and then finally separated into fast and slow lane columns. The rows correspond to station numbers within the test section. Additionally, each table includes a section summary which provides totals from each column. Table 12 and Table 13 are examples of these individual tables from within the Excel file.

For each test section, the data from the summary sections were combined into PSR calculation tables within the Excel spreadsheet. PSR values were calculated separately for Fast and Slow Lanes within each of the 32 test sections for each survey. Examples of a PSR calculation table for one lane of an asphalt and concrete section are presented in Table 14 and Table 15 respectively

Any concerns regarding categorization, definition, and weighting of distresses that arose during this process were noted. Assumptions pertaining to the interpretation of survey diagrams and vague areas within the Special Provision, Section 490 spec were also noted along the way. Table 14 and Table 15 show an interim set of weight factors for each distress/severity level. The weight factors were extensively evaluated during the research as part of the iterative process for establishing the WVDOH PSR calculation methodology. 


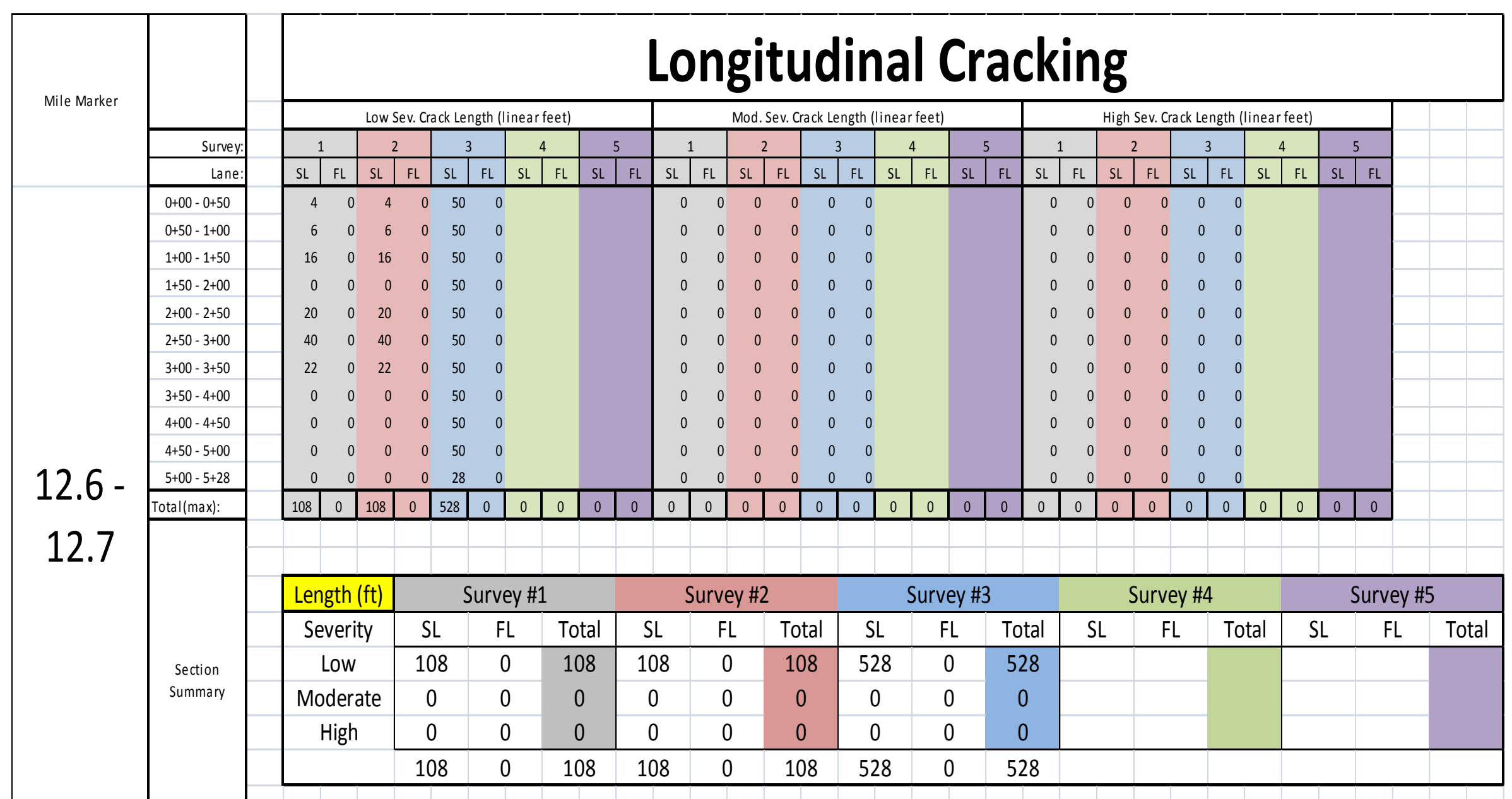

Table 12: Longitudinal Cracking within NB 12.6-12.7 (HMA Section) 


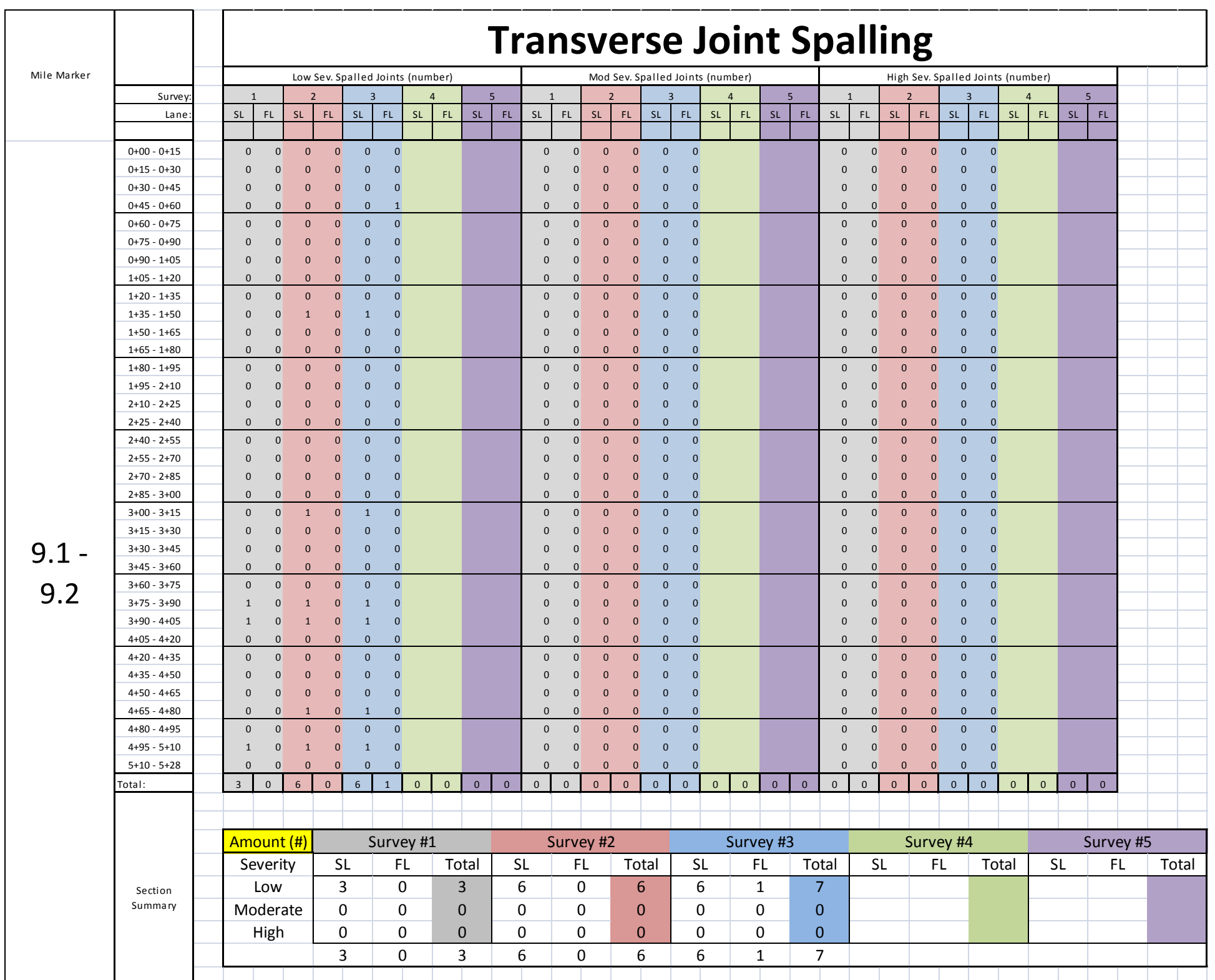

Table 13: Transverse Spalled Joints within NB 9.1-9.2 (PCC Section) 


\section{Slow Lane Survey 3}

\begin{tabular}{|c|c|c|c|c|c|c|c|c|}
\hline PSR Distresses & Severity & Weighting Factor & How to Measure (S.P.) & Number & Length & Percent & Individual Weighted Distress & \\
\hline \multirow{3}{*}{ Transverse Cracking } & Low & 0.01 & Count & 0 & - & 0.00 & 0.00 & \\
\hline & Medium & 0.10 & Count & 0 & - & 0.00 & 0.00 & \\
\hline & High & 0.20 & Count & 0 & - & 0.00 & 0.00 & \\
\hline \multirow{3}{*}{ Longitudinal Cracking } & Low & 0.02 & Linear Feet & - & 0 & 0.00 & 0.00 & \\
\hline & Medium & 0.03 & Linear Feet & - & 0 & 0.00 & 0.00 & \\
\hline & High & 0.04 & Linear Feet & - & 0 & 0.00 & 0.00 & \\
\hline \multirow{3}{*}{$\begin{array}{l}\text { Longitudinal Joint } \\
\text { Deterioration }\end{array}$} & Low & 0.04 & Linear Feet & - & 316 & 59.85 & 2.39 & \\
\hline & Medium & 0.05 & Linear Feet & - & 0 & 0.00 & 0.00 & \\
\hline & High & 0.06 & Linear Feet & - & 0 & 0.00 & 0.00 & \\
\hline \multirow{3}{*}{ Block Cracking } & Low & 0.05 & Linear Feet & - & 0 & 0.00 & 0.00 & \\
\hline & Medium & 0.10 & Linear Feet & - & 0 & 0.00 & 0.00 & \\
\hline & High & 0.15 & Linear Feet & - & 0 & 0.00 & 0.00 & \\
\hline \multirow{3}{*}{$\begin{array}{l}\text { Alligator/Fatigue } \\
\text { Cracking }\end{array}$} & Low & 0.15 & Linear Feet & - & 0 & 0.00 & 0.00 & \\
\hline & Medium & 0.25 & Linear Feet & - & 0 & 0.00 & 0.00 & \\
\hline & High & 0.35 & Linear Feet & - & 0 & 0.00 & 0.00 & \\
\hline \multirow{2}{*}{ Rutting } & Low & 0.04 & Linear Feet & - & 0 & 0.00 & 0.00 & \\
\hline & High & 0.15 & Linear Feet & - & 0 & 0.00 & 0.00 & \\
\hline Raveling/Weathering & None & 0.02 & Linear Feet & - & 528 & 100.00 & 2.00 & \\
\hline \multirow[t]{3}{*}{ Patching } & None & 0.04 & Linear Feet & - & 0 & 0.00 & 0.00 & \\
\hline & & & & & & & 4.39 & TWD \\
\hline & & & & & & & 82.0 & PSR \\
\hline
\end{tabular}

Table 14: Post Survey 3 PSR Calculator for Slow Lane of NB 13.2-13.3 (HMA Section)

\begin{tabular}{|c|c|c|c|c|c|c|c|c|c|}
\hline PSR Distresses & Severity & Weighting Factor & How to Measure (S.P.) & Number of Joints & Number of Panels & Length & Percent & Individual Weighted Distress & \\
\hline \multirow{3}{*}{$\begin{array}{l}\text { Transverse Joint } \\
\text { Spalling }\end{array}$} & Low & 0.15 & Number of Joints & 21 & - & - & 60.00 & 9.00 & \\
\hline & Medium & 0.20 & Number of Joints & 0 & - & - & 0.00 & 0.00 & \\
\hline & High & 0.25 & Number of Joints & 0 & - & - & 0.00 & 0.00 & \\
\hline \multirow{3}{*}{ Faulted Joints } & Low & 0.15 & Number of Joints & 0 & - & - & 0.00 & 0.00 & \\
\hline & \begin{tabular}{|l|} 
Medium \\
\end{tabular} & 0.20 & Number of Joints & 0 & - & - & 0.00 & 0.00 & \\
\hline & High & 0.25 & Number of Joints & 0 & - & - & 0.00 & 0.00 & \\
\hline \multirow{2}{*}{ Corner Breaks } & Low & 0.14 & Number of Panels & - & 0 & - & 0.00 & 0.00 & \\
\hline & High & 0.25 & Number of Panels & - & 0 & - & 0.00 & 0.00 & \\
\hline \begin{tabular}{|l|} 
Faulted Panels \\
(Transverse Crack)
\end{tabular} & None & 0.15 & Number of Panels & - & 0 & - & 0.00 & 0.00 & \\
\hline Patched Panels & None & 0.14 & Number of Panels & - & 0 & - & 0.00 & 0.00 & \\
\hline \multirow{3}{*}{$\begin{array}{l}\text { Longitudinal Joint } \\
\text { Spalling }\end{array}$} & Low & 0.15 & Number of Panels & - & 0 & - & 0.00 & 0.00 & \\
\hline & \begin{tabular}{|l|} 
Medium \\
\end{tabular} & 0.20 & Number of Panels & - & 0 & - & 0.00 & 0.00 & \\
\hline & High & 0.25 & Number of Panels & - & 0 & - & 0.00 & 0.00 & \\
\hline Localized Distress & None & 0.40 & Number of Panels & - & 0 & - & 0.00 & 0.00 & \\
\hline \multirow{3}{*}{ Transverse Cracks } & Low & 0.10 & Number of Panels & - & 0 & - & 0.00 & 0.00 & \\
\hline & Medium & 0.18 & Number of Panels & - & 0 & - & 0.00 & 0.00 & \\
\hline & High & 0.25 & Number of Panels & - & 0 & - & 0.00 & 0.00 & \\
\hline \multirow{3}{*}{ Longitudinal Cracks } & Low & 0.10 & Number of Panels & - & 2 & - & 5.71 & 0.57 & \\
\hline & \begin{tabular}{|l} 
Medium \\
\end{tabular} & 0.18 & Number of Panels & - & 0 & - & 0.00 & 0.00 & \\
\hline & High & 0.25 & Number of Panels & - & 0 & - & 0.00 & 0.00 & \\
\hline Pop Outs & None & 0.04 & Number of Panels & - & 0 & - & 0.00 & 0.00 & \\
\hline \multirow[t]{3}{*}{ Scaling } & None & 0.02 & Linear Feet & - & - & 0 & 0.00 & 0.00 & \\
\hline & & & & & & & & 9.57 & TWD \\
\hline & & & & & & & & 65.0 & PSR \\
\hline
\end{tabular}

Table 15: Post Survey 3 PSR Calculator for Slow Lane of NB 9.4-9.5 (PCC Section) 


\subsection{ARAN Surface Distress Data Collection and Comparison Process}

The ARAN recorded surface distresses for the state's pavement management system in the fall of 2012 between Survey 2 and 3. The ARAN data provides another form of surface distress data which could be beneficial to the analysis of the manually surveyed data.

A comparison was conducted to determine the feasibility of replicating indices outlined within the Composite Condition Index using manually surveyed distresses and the $\mathrm{CCl}$ calculation model. This was a quick investigation into the consistency between ARAN and human recorded surface distresses for US 35. Inconsistencies between distress and severity definitions were noted and additional assumptions and concerns would be identified from this comparison.

After all assumptions, concerns, and inconsistencies were addressed, distress data from the northbound slow lane in Survey 1, 2, and 3 were applied to the equations for each of the indices within the PMS document. All three calculated indices were compared graphically to the index value provided by the WVDOH PMS.

The above analysis provides a comparison of the PMS statistics computed with the manual survey to the indices in the PMS database. It was not possible to reverse this process to compute PSR from the data in the PMS database. The distress types, extent and severity in the PMS database are not compatible with the calculation of PSR.

\subsection{Deflection Testing Data Collection and Analysis Process}

In July of 2014, FWD deflection data were collected for US 35 in both northbound and southbound slow lanes of asphalt and concrete sections. The West Virginia Division of Highways does not currently rely on FWD deflection data for managing or designing its pavements, so a goal of this research was to investigate the potential for doing so in the future. Hence, no LTPP, ASTM, or other FWD procedure was explicitly followed with regards to location and frequency of FWD tests.

This section has five parts. The first describes the process by which deflection tests were performed. The second section describes the initial screening of the FWD data. The following three, describe the method by which three different computer programs were used to analyze the data collected. The three programs, which are described in the Literature Review, are MODULUS 6.1, MODTAG, and BAKFAA. 


\subsubsection{Deflection Data Collection}

At each test location, three drops were performed at four target load levels $(6,9,12$, and 16 kips). The sensors were placed at $-12,0,8,12,18,24,36,48$, and 60 inches from the center of the load. The -12 and 8 inch sensor were not used for analysis.

All FWD tests were performed at the transverse center of the slow lane of each direction. For the asphalt sections, FWD tests were performed approximately at the beginning and end of each section, resulting in 36 test locations. On concrete sections the FWD tests were performed at approximately the longitudinal center of the first and last whole slab of each section resulting in 28 test locations.

\subsubsection{Initial Screening of Deflection Data}

Due to the stiffness of concrete pavement it is recommended that only drops within a 16 kip load level be analyzed. Smaller load levels do no generate large enough deflections to analyze the structural capacity. Additionally, per the LTPP protocol, deflection data should be collected at the longitudinal center of the slab $+/-10 \%$ of the slab length. Using these criteria, 12 of the 28 test locations were eliminated from the analysis. The data was then checked to ensure the measured deflections consistently decreased with an increase in distance from the load center. These criteria eliminated 15 drops of the remaining eligible 48 drops (16 acceptable test locations with 3 acceptable drop loads). The screening process left 33 deflection tests for backcalculation analysis within the concrete section.

The longitudinal location criteria and load level criteria do not apply to asphalt pavements. The decreasing deflection screening was applied to the asphalt section. All of the deflection basins exhibited deflections which decreased as distance from the center of the load increased.

\subsubsection{MODULUS 6.1 Deflection Data Analysis Process}

The MODULUS 6.1 program is limited to the analysis of asphalt pavements. Two separate files for US 35 north and southbound asphalt sections were imported into MODULUS 6.1. Both files included all 12 drops at the 36 asphalt test locations. Backcalculation analyses were performed using each file, one assuming a three layer pavement structure, the other assuming two layers. An average temperature of $87^{\circ} \mathrm{F}$ was used; the surface temperature of the pavement varied significantly between morning and afternoon measurements. This temperature effectively restricts asphalt layer moduli to a range of 120 to $660 \mathrm{ksi}$. 
The three layer system had a "Surface" asphalt layer of 12.75 inches and Poisson's Ratio of 0.40 followed by, a "Base" layer consisting of "Other Material" of four inch thickness with moduli range of 10 to $150 \mathrm{ksi}$ and Poisson's Ratio of $\mathbf{0 . 3 5}$, followed by a semi-infinite "Subgrade" layer consisting of "Other Material" with a seed modulus of $10 \mathrm{ksi}$ and Poisson's Ratio of 0.40 . After running the backcalculation, MODULUS 6.1 provided outputs for each individual deflection basin. Moduli values for each of the three layers, an estimated depth to bedrock, and an absolute error per sensor were recorded for all 432 instances.

From there, the VDOT subgrade modulus "rule-of-thumb" was applied and two different sets of data were generated. The first was the program output subgrade modulus, $M_{R}$, and the second divided the $M_{R}$, by three and capped it at $10 \mathrm{ksi}$. This will be referred to as $M_{R^{*}}$. For both $M_{R}$ data sets, an effective modulus for total pavement structure, $E_{p}$, was then calculated as described in the literature review. This required a Microsoft Excel Macro to simultaneously Goal Seek the 432 unique instances in both subgrade modulus sets. After that, the AASHTO 93 effective structural number equation described in the literature review provided an effective structural number, $\mathrm{SN}_{\text {eff, }}$ for all 864 data sets and the AASHTO 1993 flexible pavement structural design equation was used to calculate an amount of equivalent single axle loads, ESAL, until failure (also known as $W_{18}$ ) for each case. All of these outputs could be averaged per the 36 test locations to compare to each other, or averaged further in the north and southbound sections entirely which correspond to morning and afternoon testing This could provide information as to the effects of using a single average pavement temperature for the entire days testing.

The two layer system had a surface layer of 16.75 inches with a Poisson's Ratio of 0.40 on a semi-infinite subgrade layer consisting of "Other Material" with a seed modulus of $10 \mathrm{ksi}$ and Poisson's Ratio of 0.40 . The same outputs were provided as in the three layer system, but this time the surface layer moduli could be considered the $E_{p}$. Additionally two more variations of $E_{p}$ were calculated as in the three layer system. In these cases, the specified equation in combination with the Excel Macro and Goal Seek function provide $E_{p}$ values for the provided $M_{R}$ and the VDOT "rule-of-thumb" $M_{R^{*}}$. All other calculations were performed as in the three layer system and thus three more sets of $\mathrm{SN}_{\text {eff }}$ and $\mathrm{W}_{18}$ were rendered.

Thus a total of five variations of $\mathrm{SN}_{\text {eff }}$ were computed for every FWD drop on the asphalt sections: 
- $\mathrm{SN}_{\text {eff }}$ as a function of $\mathrm{E}_{\mathrm{p}}$ as a function of $\mathrm{M}_{\mathrm{R}}$ from a 3 layer MODULUS 6.1 backcalculation

- $\mathrm{SN}_{\text {eff }}$ as a function of $\mathrm{E}_{\mathrm{p}}$ as a function of $\mathrm{M}_{\mathrm{R}^{*}}$ from a 3 layer MODULUS 6.1 backcalculation

- $\mathrm{SN}_{\text {eff }}$ as a function of $\mathrm{E}_{\mathrm{p}}$ from a 2 layer MODULUS 6.1 backcalculation ( $\mathrm{E}_{\mathrm{p}}$ output by MODULUS 6.1)

- $S N_{\text {eff }}$ as a function of $E_{p}$ and $M_{R}$ from a 2 layer MODULUS 6.1 backcalculation

- $\mathrm{SN}_{\text {eff }}$ as a function of $\mathrm{E}_{\mathrm{p}}$ and $\mathrm{M}_{\mathrm{R}^{*}}$ from a 2 layer MODULUS 6.1 backcalculation

\subsubsection{MODTAG Deflection Data Analysis Process}

As in the MODULUS 6.1 analysis, the files for US 35 were imported into MODTAG. A three layer backcalculation was performed using each file. All layer inputs were the same as in MODULUS 6.1 with the exception that MODTAG uses seed moduli values rather than ranges. These were input as 400 and $75 \mathrm{ksi}$ for the surface and base layers. The built in temperature correction was overridden and the pavement was set to a uniform temperature of $87^{\circ} \mathrm{F}$ to make the results comparable to the MODULUS 6.1 outputs.

MODTAG output $\mathrm{M}_{\mathrm{R}}, \mathrm{SN}_{\text {eff, }}$ and $\mathrm{W}_{18}$ for each load level of each test location. These outputs were averaged values of the three drops at each load level. In the asphalt section, a total of 144 sets of deflection data were available from the 36 test locations. As with MODULUS 6.1, $M_{R^{*}}$ was included in the analysis. The same general analysis processes were used to develop three different $\mathrm{SN}_{\text {eff }}$ values for the pavement which could be defined as:

- $\mathrm{SN}_{\text {eff }}$ as provided by 3 layer MODTAG backcalculation

- $S N_{\text {eff }}$ as a function of $E_{p}$ and $M_{R}$ from a 3 layer MODTAG backcalculation

- $S N_{\text {eff }}$ as a function of $E_{p}$ and $M_{R^{*}}$ from a 3 layer MODTAG backcalculation

MODTAG was not used for the analysis of the concrete pavements as MODTAG treats the three replicate drops as a single test result and none of the data sets had all three drops pass the screening process.

\subsubsection{BAKFAA Deflection Data Analysis Process}

BAKFAA was used to perform analysis on both asphalt and concrete FWD data. Although BAKFAA is not necessarily designed specifically for rigid pavement backcalculations, the linear elastic analysis subroutine for estimating layer moduli is applicable to rigid pavements provided appropriate seed moduli are used. In the three layer asphalt backcalculation, the same inputs from MODTAG were 
used in BAKFAA, with the addition of interface coefficients of 1.0 (assuming complete bondage between layers), and the exclusion of any temperature input because BAKFAA performs no temperature correction. BAKFAA also included a semi-infinite bedrock layer which required inputs for modulus and depth. These would not be recalculated by the program so values of $500 \mathrm{ksi}$ and 200 inches from pavement surface were used. The depth to bedrock was estimated at 200 for the sake of reasonable comparison to MODULUS 6.1 and MODTAG which previously estimated average depth to hard bottom of 239 and 188 inches respectively. Deflection data from the third replicate of the 16 kip load level at each asphalt test location was manually input into BACKFAA which provided backcalculated moduli values for all three layers. In all, 36 sets of layer moduli were output. As with the previous programs, $M_{R}$ and $M_{R^{*}}$ would be used to generate two sets of $S N_{\text {eff }}$ for each deflection test.

The same input process was used for 33 concrete drops. The exception being surface and base layers had depths of 10 and 6 inches respectively and the surface layer had seed modulus and Poisson's ratio inputs of $4000 \mathrm{ksi}$ and 0.15 respectively. The only results produced with this analysis were estimates of $M_{R}$ for some of the concrete sections. These were compared to the $M_{R}$ results for the asphalt sections. 


\section{Chapter 4. Analysis}

\subsection{Analysis of Improvements in Pavement Surface Rating Process}

The improvement of the PSR calculation process was iterative and continuously evolving. This section documents the problems and solutions encountered throughout as comprehensively as possible. It is separated into two parts. The first outlines the initial analysis done after the third survey was completed in July of 2013. The problems discussed there are generally concerning definitions of distresses. Eliminating vagueness and establishing rules for collecting distress data were critical to progressing with improvements. The second part deals with the improvement of PSR in terms of correlation to $\mathrm{PCl}$ while also fixing any unresolved flaws in the logic and organization of the process.

\subsubsection{Initial Analysis of US 35 PSR}

The first analysis of manually surveyed surface distress data occurred after the third survey in July of 2013. PSR was calculated based on the April 2012 version of "West Virginia Department of Transportation Division of Highways Special Provision, Section 490: Nine Year Pavement Performance Criteria". The method of calculating PSR would be slightly modified by the time of the fifth survey in May of 2014 and completely overhauled after that point. The Initial PSR calculations for US 35 are now obsolete and are only included to mark the progression of changes that were made. A comparison of fifth survey PSR's using the initial and final calculation methods are prevented later.

Assumptions made during the initial collection of distress data and calculation of PSR for asphalt sections include:

- Only Transverse Cracking, Longitudinal Cracking, Joint Deterioration, Block Cracking, Alligator (Fatigue) Cracking, Rutting, Raveling/Weathering, and Patching are currently factored into PSR.

- Bleeding and Potholes and any other distresses noticed are marked on the surveys but are not factored into PSR.

- Cracking noted at joints is counted as both Longitudinal Cracking and Joint Deterioration

- Joints labeled "LS ravel/deterioration", "slight ravel/deterioration", "Slight raveling at center joint" are quantified as joint deterioration, not longitudinal cracking.

- Joints labeled "LS JT", "LS paint stripe" are considered both Longitudinal Cracking and Joint Deterioration.

- The center joint is included in the slow lane as it is located a few inches to the slow lane side of the lane divider for the vast majority of the asphalt section of US 35.

- $\quad$ Any cracks with unlabeled severity are considered Low Severity. 
- When the first page of a sections survey is labeled "raveling 100\%", "100\% raveled", "slight raveling/deterioration", "LS raveling throughout", "generally open/raveled", "opened slightly center mat", "generally slightly open texture", "center raveling full length", etc. without specifically referring to a joint, it means entire length is raveled/weathered.

- Cases where a distress is noted on a survey and then crossed out on the following survey, the distress is still included in the original survey.

- Pencil lines indicate first survey, red ink indicates second survey, blue ink indicates third survey except on mile marker 15.5-15.6 where the first survey is recorded in blue ink, and the third was recorded in pencil (excluding the rutting recordings which were done in the correct color).

- Average Rutting Depth was determined by averaging all values recorded in both wheel paths within the same lane.

Below is the list of necessary assumptions made during the initial collection of distress data and calculation of PSR for concrete sections:

- Only Transverse Joint Spalling, Faulted Joints (longitudinal and transverse), Corner Breaks, (Faulted Panels, Patched Panels, Longitudinal Joint Spalling, Localized Distress, Transverse Cracking, Longitudinal Cracking, and Scaling are currently factored into PSR.

- Pop outs and any other distresses noticed are marked on the surveys but are not factored into PSR calculation. (This was assumed the time of the third survey data collection but was changed before the first data analysis occurred.)

- The center joint is included in the slow lane as it is located a few inches to the slow lane side of the lane divider for the vast majority of the concrete section of US 35.

- Any unlabeled cracks are considered Low Severity.

- All unlabeled spalling as well as spalling labeled "beginning spalling" are counted as Low Severity.

- For Transverse Joint distresses, the slab that begins with that joint (from left to right on the surveys) is the slab where the distress is recorded.

- Vaguely labeled spalling widths such as "W:4", are recorded using discretion of drawing and assumed meaning.

- Corner Breaks are determined based on the size requirements and the apparent size drawn on surveys.

- Moderate and High Severity Corner Breaks fall under the High severity distress weight.

- Hairline shrinkage cracks which do not propagate far into a slab that are perpendicular to transverse joints and near slab edges may be recorded on diagrams but are not counted as longitudinal cracks.

Below is a list of additional concerns regarding the survey procedure, the data which were collected, and the process for calculating PSR: 
- $\quad$ Surveys for PCC Northbound lanes between mile marker 11.7 and 11.8 have many discrepancies between the 3 surveys.

- PSR per "West Virginia Department of Transportation Division of Highways Special Provision" does not currently include pop outs or several other distresses included in the LTPP distress identification manual. (This was true at the time of the third survey but was changed before the initial analysis of the data.)

- The parameters for all cracks in both pavement types are very specific especially in the differences between severity levels. This can lead to distress being classified differently between two surveys depending on the ability of the surveyor to identify if an observation was above or below the threshold.

- No parameters are included for severities of joint faulting for PCC jointed pavements anywhere in the Section 490, S.P. document or in LTPP however there are 3 severities included in the PSR calculation.

- The difference between Longitudinal Cracking and Alligator Cracking within the Longitudinal Cracking definition in the Section 490, S.P. document is very vague.

- Because there are technically two longitudinal joints in the slow lanes of both pavements, distresses involving longitudinal joints can have up to $200 \%$ distress in the PSR calculation which seems strange but is shown as acceptable in an example in the Section 490, S.P. document.

- The Section 490, S.P. document gives only two Severity distress weight values for Corner Breaks. It defaults to the LTPP parameters for these severities; however, LTPP defines Corner Breaks as having 3 severities.

- The surveys are conducted by several different people within each survey and within each $1 / 10^{\text {th }}$ mile section. Discrepancies pertaining to labeling and nomenclature on the surveys are evident.

- This entire process relies on the dependability and training of the surveyors. If surveys are incorrect, PSR will be incorrect.

\subsubsection{Refinement of PSR Calculation Procedure}

An iterative process of changing weight factors was initially used in the recalibration of the PSR calculator. This was guided mostly by judgment of senior engineers from the $\mathrm{DOH}$ and advisors. As the $4^{\text {th }}$ and $5^{\text {th }}$ surveys were conducted in September 2013 and May 2014, more distresses began to emerge and noticeable progressions in certain distresses also became evident. As the road aged and distresses increased, the observed test section Total Weighted Distress values increased, and calculated PSR values decreased, and the effects of the applied weight factors became more evident.

Several variations to weight factors were made so PSR converged toward values which reflected the distresses across US 35. With each variation, additional distress combinations were considered because the road still was relatively young and did not display a wide enough variation of distresses. Several changes to distress definitions, additions of distresses, and combinations of other distresses were made to remedy concerns within the process. 
PSR values which were representative of the deterioration of the pavement condition had been reached and the effect of distresses on calculated PSR was intuitively understood but the process was still subjective and imprecise. A more definite and unarguable approach was desired so the roots of the PSR calculation process were revisited. $\mathrm{PCl}$ as developed by the U.S. Army Corps of Engineers and describe in ASTM D6433-11 "Standard Practice for Roads and Parking Lots Pavement Condition Index (PCI) Survey" is still one of the most developed and accepted pavement rating systems. PCI had a major influence on the development of WVDOH's PSR. Because PCl is so well defined and serves the same purpose as PSR, an attempt to directly correlate PSR to PCI was made.

In order to accomplish this, a complete understanding of the $\mathrm{PCl}$ calculation process is necessary. The process is summarized within the U.S. Army Corps of Engineers Pavement Condition Index section of Chapter 2. To accurately correlate PSR and PCl, the distress weight factor for PSR must reflect the distress deduct curve for that distress. Several aspects must be considered here due to the differences in the two processes and the goal of WVDOH in creating a process simpler than that used for $\mathrm{PCl}$.

1. PSR uses a single distress weight factor which can be multiplied by a distress extent. This weight factor assumes there is a linear relationship between the extent of a distress and the effect on the pavement condition. $\mathrm{PCl}$ uses a deduct curve for all distresses where the distress weight is dependent on the extent. This relationship is not linear and in nearly all cases displays a logarithmic trend where the change in deduct lessens as extent increases toward $100 \%$. Because of this difference, PSR cannot possibly perfectly match a PCl curve.

2. In many cases PSR uses linear measurements or a counted amount to quantify a distress and determine an extent. PCl uses a percent area affected to determine extent in nearly all cases. PSR works under the assumption that a distress extent of $100 \%$ is possible. That is to say, a longitudinal joint within an asphalt section which expresses low severity deteriorated for 528 feet within a $1 / 10^{\text {th }}$ mile section would justify a recording of $100 \%$ low severity joint deterioration toward a PSR calculation. The same section when analyzed with $\mathrm{PCl}$ would qualify as approximately $8 \%$ low severity joint deterioration (if the lane width is $12 \mathrm{feet}$ ). Within $\mathrm{PCl}$ many distresses simply cannot reach an extent of $100 \%$. For example, by PCl's definition, $100 \%$ longitudinal cracking would mean a longitudinal crack is present within each foot of lane width for the length of the test section. At this point, this distress would likely not be considered longitudinal cracking. This requires a case by case comparison when attempting to correlate each individual distress. 
3. In both $\mathrm{PCl}$ and PSR, some distress combinations are not possible. For instance, in either case, a theoretical asphalt section with 50\% Block Cracking cannot possibly also have $75 \%$ Longitudinal Cracking because regardless how these distresses are mapped on a section, there would be overlap and by definition Block Cracking would override Longitudinal Cracking. For this reason, if a comprehensive correlation of PCl and PSR can be accomplished, theoretical distress combinations must be derived from an observable scenario before $\mathrm{PCl}$ or PSR is calculated. It would be erroneous to calculate each across the array of mathematically possible distress extents. The practical range of distresses requires understanding of each system as well as experience in observing surface distresses.

4. $\mathrm{PCl}$ requires a correction factor after combining all distresses. This reduces the total deduct based on the total amount of observed distresses. The idea here is that there is a type of diminishing return occurring. As the amount of different distresses increase, the effect of each individual one is reduced. Similar to point \#1 above, PSR assumes a linear relationship and in this case has no reduction procedure. Where $\mathrm{PCl}^{\prime}$ c combination of distresses will express a logarithmic trend again, PSR will have to attempt to linearly approximate the curve.

5. There is a concern that within the PCl calculation method, Jointed Plain Concrete Pavement (JPCP) sections can only be optimally modeled with $\mathrm{PCl}$ if there are exactly 20 slabs. An attempt to normalize the PSR to any reasonable amount of slabs within $1 / 10^{\text {th }}$ of a mile must be made.

6. When making changes to PSR, maintaining a balance of accuracy in modeling pavement condition and simplicity as compared to $\mathrm{PCl}$ is critical. The main reason for developing PSR is that $\mathrm{PCl}$ is requires too many calculations and references to graphs and charts. Approximating $\mathrm{PCl}^{\prime}$ accuracy while reducing the effort to reach it is the goal.

7. It should also be stressed that the goal is not to have any given distress scenario result in identical values for PSR and PCI. The goal is to develop a PSR scale that contains a correlation to $\mathrm{PCl}$ but not necessarily at a 1:1 ratio. If the ranges for the qualitative condition expressions need to be adjusted they can be.

8. Additionally, the goal here is to create a scale that can compare asphalt and concrete sections. $\mathrm{PCl}$ claims that their pavement condition scale is the same for both asphalt and concrete surfaces. This is to say that a $\mathrm{PCl}$ of 75 represents the same quality road surface regardless of pavement type. So if there is a correlation between PSR and PCl, there should be a goal of mirroring that correlation between asphalt and concrete sections. 
9. Another goal is to adapt the asphalt PSR calculator to account for composite pavements. This would make the PSR calculation method dependant on the pavement surface material. Although composite pavements are technically classified as rigid pavements, the surface distresses observable would still tend more toward flexible asphalt pavement distresses. However definition changes are necessitated to accommodate this goal.

To incorporate all of these concerns while converging on an acceptable PSR calculation model, first a case by case comparison of PCI deduct curves and PSR weight factors was made. Because both PSR and PCl are based on $0-100$ scales, PSR weighted distresses can be converted into deduct values which would be comparable to $\mathrm{PCl}$ deduct values. For each severity of each distress, the original PSR weighted distress was entered alone into the Total Weighted Distress, TWD, calculation formula at several extent values within the $0-100 \%$ range.

PCl's deduct value curves for asphalt distresses show extent on a logarithmic scale and concrete distresses on a linear scale. This is because the extent of asphalt distresses is mostly measured on an area affected basis and the extent of concrete distresses is measured on an amount of slabs or joints affected. If $50 \%$ or $100 \%$ of joints within a concrete section shows spalling at some severity, then the extent would be $50 \%$ or $100 \%$ respectively. The resulting distress between these two scenarios would be noticeably different, so it is important that concrete distresses measure on a slab/joint affected basis be considered on a $0-100 \%$ extent range. This is contrary to say, Alligator/Fatigue Cracking on an asphalt surface, where the difference between 5 and $10 \%$ affected area may very well be more noticeable than the difference between 70 and $100 \%$. For this reason the array of extent values input into TWD calculations was dependent on the scale used by PCI. For logarithmic scales, the list of extents used to calculate TWD was: .1, .2, .5, 1, 2, 5, 10, 20, 50, and 100\%. For standard scales, the list of extents used to calculate TWD was: $0,5,10,15,20,30,50,70$, and $100 \%$. This process successfully converted individual PSR weight factors in deduct curves that could be compared to PCl deduct curves. Each of these extent values were entered into their corresponding deduct curves to find their deduct value. These were entered into Excel files for analyses.

From there, each severity of each distress was carefully examined. The generated deduct curves for PSR TWD deducts for each case was iteratively modified until the curve agreed with the PCl deduct curve. Again, this agreement does not mean that the $\mathrm{PCl}$ and PSR deduct values were identical for each input extent. Each case had to be customized to account for differences in distress definitions and extent calculation methods. Additionally, $\mathrm{PCl}$ 's deduct value correction factor had to be accounted for. 
The modification of the PSR deduct curve was accomplished by changing the PSR weight factor associated with each distress. The curve was updated automatically as the new weight factor and the array of extent percentages were used to recalculate the Total Weighted Distress within the Excel file.

A comprehensive analysis of the influences on each weight factor's final value is included in Appendix B. Each severity of each distress for both pavement types includes a list of factors which resulted in the final weight factor. A graphical comparison of PSR weight factor and PCI deduct curve is provide for each relevant distress type/severity combination.

After an examination of each deduct curve individually, the practicality of the combination of distresses had to be verified. To start, a few asphalt and concrete sections from US 35's fifth survey were sampled. PSR for these sections was calculated with the new distress weight factors. Then PCI was computed for each. Only a few US 35 sections were used because the combination and variation of distress types and amounts was fairly limited. From there, hypothetical sections were developed that encompassed each distress type and severity as a primary distress within a section. These hypothetical test sections used logical and realistic combinations of supporting distresses in some cases. Each time, the hypothetical section scenario was developed, and PSR and PCI were calculated. Often, these scenarios were based on interesting pavement distress types and combinations observed by engineers at WVDOH.

A PCI vs. PSR correlation for 77 unique hypothetical section scenarios was developed. Of these, 39 were asphalt surface sections and 38 were concrete surface sections. Regression lines were generated for the PCI vs. PSR correlations of both surface types. A comparison of these regression lines provided a means to determining consistency between PSR values of asphalt versus concrete.

One more series of alterations to PSR weight factors was then made. For both correlation graphs, groups of outliers were identified based on the common distress within them that was affecting the best fit line. As slight corrections were made to the weight factors, PSR of these groupings converged toward the rest of the test points. As the envelopes of both correlations began to tighten and the best fit lines began to converge toward each other, the weight factors were reviewed to ensure that they were still logically sound and satisfied all other concerns mentioned previously. This process repeated until all concerns were satisfied and a panel of engineers from the WVDOH approved the result. 


\subsection{Comparison Analysis of ARAN and Manual Survey Surface Distress Data}

As stated within Chapter 3, an attempt at replicating the indices associated with $\mathrm{CCl}$ (outlined in Chapter 2) was made using the surface distress data collected during the first three manual surveys. This was to be compared to the data collected by an ARAN and analyzed by the PMS dTIMS software by the WVDOH.

Distress data were observed within the slow lane of the northbound section of US 35 by the ARAN in a Fall 2012 survey. Data were provided for the entire length of the highway. However, this data set was reduced to only include sections that were surveyed manually for calculation of PSR.

A summary of the distress data recorded within the asphalt section, concrete section, and a table identifying the labels used with the Pavement Management System is presented in Table 16, Table 17, and Table 18 respectively. The important information provided within these tables is the columns labeled PSI, SCl, ECl, RDI, JCl, and CSI. As explained in Chapter 2, $\mathrm{CCl}$ is recorded as the lowest of all other indices calculated for a section. PSI was the lowest calculated index for every section within the study. PSI is based solely on IRI which is a measure of surface roughness and cannot be determined via a manual surface distress survey. The remaining indices were calculated using the data collected from Section 490, Special Provision manual surveys and compared to the values provided by the Pavement Management System. 


\begin{tabular}{|c|c|c|c|c|c|c|c|c|c|c|c|c|c|c|c|c|c|c|c|}
\hline BEG_MP & END_MP & IRI_MEAN & PSI & $\mathrm{SCl}$ & $\mathrm{ECl}$ & RDI & $\mathrm{CCl}$ & FALLIG_L & FALLIG_M & FALLIG_H & FLONG_L & FLONG_M & FLONG_H & FTRANS_L & FTRANS_M & FTRANS_H & FBLOCK_L & FBLOCK_M & FBLOCK_H \\
\hline 12.60 & 12.70 & 146.800 & 2.996 & 5.000 & 5.000 & 4.777 & 2.996 & 0 & 0 & 0 & 0 & 0 & 0 & 0 & 0 & 0 & 0 & 0 & 0 \\
\hline 13.20 & 13.30 & 53.800 & 4.012 & 5.000 & 5.000 & 4.874 & 4.012 & 0 & 0 & 0 & 0 & 0 & 0 & 0 & 0 & 0 & 0 & 0 & 0 \\
\hline 13.80 & 13.90 & 57.400 & 3.955 & 5.000 & 5.000 & 4.874 & 3.955 & 0 & 0 & 0 & 0 & 0 & 0 & 0 & 0 & 0 & 0 & 0 & 0 \\
\hline 14.40 & 14.50 & 65.200 & 3.833 & 5.000 & 5.000 & 4.741 & 3.833 & 0 & 0 & 0 & 0 & 0 & 0 & 0 & 0 & 0 & 0 & 0 & 0 \\
\hline 14.60 & 14.70 & 58.600 & 3.932 & 5.000 & 5.000 & 4.704 & 3.932 & 0 & 0 & 0 & 0 & 0 & 0 & 0 & 0 & 0 & 0 & 0 & 0 \\
\hline 15.20 & 15.30 & 102.300 & 3.327 & 5.000 & 5.000 & 4.777 & 3.327 & 0 & 0 & 0 & 0 & 0 & 0 & 0 & 0 & 0 & 0 & 0 & 0 \\
\hline 15.40 & 15.50 & 81.300 & 3.615 & 5.000 & 5.000 & 4.741 & 3.615 & 0 & 0 & 0 & 0 & 0 & 0 & 0 & 0 & 0 & 0 & 0 & 0 \\
\hline 16.00 & 16.10 & 59.900 & 3.914 & 5.000 & 5.000 & 4.665 & 3.914 & 0 & 0 & 0 & 0 & 0 & 0 & 0 & 0 & 0 & 0 & 0 & 0 \\
\hline 16.40 & 16.50 & 115.800 & 3.239 & 4.790 & 5.000 & 4.005 & 3.239 & 1 & 0 & 0 & 0 & 0 & 0 & 0 & 0 & 0 & 0 & 0 & 0 \\
\hline
\end{tabular}

Table 16: Summary of WVDOH PMS Distress Data for US 35 Northbound Slow Lane Asphalt Section

\begin{tabular}{|c|c|c|c|c|c|c|c|c|c|c|c|c|c|c|c|}
\hline BEG_MP & END_MP & IRI_MEAN & PSI & $\mathrm{JCl}$ & $\mathrm{CSI}$ & JFAULT_L & JFAULT_M & JFAULT_H & JNT_DISTT & BRSLBTR_L & BRSLBTR_M & BRSLBTR_H & BRSLBTRL_L & BRSLBTRL_M & BRSLBTRL_H \\
\hline 9.1 & 9.2 & 81.000 & 3.598 & 5.000 & 5.000 & 0 & 0 & 0 & 0 & 0 & 0 & 0 & 0 & 0 & 0 \\
\hline 9.4 & 9.5 & 69.900 & 3.756 & 5.000 & 5.000 & 0 & 0 & 0 & 0 & 0 & 0 & 0 & 0 & 0 & 0 \\
\hline 10.4 & 10.5 & 66.200 & 3.817 & 5.000 & 5.000 & 0 & 0 & 0 & 0 & 0 & 0 & 0 & 0 & 0 & 0 \\
\hline 10.8 & 10.9 & 62.600 & 3.872 & 5.000 & 5.000 & 0 & 0 & 0 & 0 & 0 & 0 & 0 & 0 & 0 & 0 \\
\hline 11.4 & 11.5 & 83.700 & 3.553 & 4.870 & 5.000 & 1 & 0 & 0 & 0 & 0 & 0 & 0 & 0 & 0 & 0 \\
\hline 11.7 & 11.8 & 63.900 & 3.851 & 5.000 & 5.000 & 0 & 0 & 0 & 0 & 0 & 0 & 0 & 0 & 0 & 0 \\
\hline 12.2 & 12.3 & 101.500 & 3.416 & 4.870 & 5.000 & 1 & 0 & 0 & 0 & 0 & 0 & 0 & 0 & 0 & 0 \\
\hline
\end{tabular}

Table 17: Summary of WVDOH PMS Distress Data for US 35 Northbound Slow Lane Concrete Section 


\begin{tabular}{|c|c|c|c|}
\hline Starting Mile Point & BEG_MP & miles & WVDOT Beginning Mile point \\
\hline Ending Mile Point & END_MP & miles & WVDOT Ending Mile point \\
\hline Roughness Average & IRI_MEAN & inches/mile & Average of Left and Right IRI (-1 - invalid) \\
\hline Present Serviceability Index & PSI & - & Present Serviceability Index $\left(\mathrm{PSI}=5^{*} \mathrm{E}^{\wedge}\left(-0.0041^{*} \mathrm{IRI}\right)\right) \quad(-1-$ invalid $)$ \\
\hline Joint Faulting Low & JFAULT_L & $\%$ & Joint Faulting - Low Severity (\% of slabs exhibiting faulting) $+/-0.1$ to 0.2 \\
\hline Joint Faulting Moderate & JFAULT_M & $\%$ & Joint Faulting - Moderate Severity (\% of slabs exhibiting faulting) $+/-0.3$ to 0.4 \\
\hline Joint Faulting High & JFAULT_H & $\%$ & Joint Faulting - High Severity (\% of slabs exhibiting faulting) $+/-0.41$ to 1.2 \\
\hline Alligator Cracking Low & FALLIG_L & & Alligator Cracking - Low Severity $\quad$ (Percentage of total wheelpah area) \\
\hline Alligator Cracking Moderate & FALLIG_M & & - Moderate Severity $\quad$ (Percentage of total wheelpah area) \\
\hline Alligator Cracking High & FALLIG_H & & (Percentage of total wheelpah area) \\
\hline Longitudinal Cracking Low & FLONG_L & & Longitudinal cracking - Low severity \\
\hline Longitudinal Cracking Moderate & FLONG_M & & Longitudinal cracking - Moderate severity $\quad$ (\% wheelpath crack affected area) \\
\hline L:ongitudinal Cracking High & FLONG_H & & Longitudinal cracking - High severity \\
\hline Transverse Cracking Low & FTRANS_L & & Transverse cracking - Low severity \\
\hline Transverse Cracking Moderate & FTRANS_M & & Transverse cracking $\quad$ - Moderate severity $\quad$ (\% lane crack affected area) \\
\hline Trans verse Cracking High & FTRANS_H & & Transverse cracking - High severity $\quad$ (\% lane crack affected area) \\
\hline Block Cracking Low & FBLOCK_L & & (Percentage of the total lane area) \\
\hline Block Cracking Moderate & FBLOCK_M & & - Moderate severity (Percentage of the total lane area) \\
\hline Block Cracking High & FBLOCK_H & & (Percentage of the total lane area) \\
\hline Joint Distress & JNT_DISTR & & (\% of slabs showing joint distress) \\
\hline Broken Slab Trans Low & BRSLBTR_L & & - Transverse only low severity $\quad$ (\% of slabs exhibiting condition) \\
\hline Broken Slab Trans Moderate & BRSLBTR_M & & - Transverse only moderate severity \\
\hline Broken Slab Trans High & BRSLBTR_H & & (\% of slabs exhibiting condition) \\
\hline Broken Slab Trans \& Long Low & BRSLBTRL_L & & - Transverse \& Longitudinal low severity \\
\hline Broken Slab Trans \& Long Moderate & BRSLBTRL_M & & - Transverse \& Longitudinal moderate severity (\% of slabs exhibiting condition) \\
\hline Broken Slab Trans \& Long High & BRSLBTRL_H & & - Transverse \& Longitudinal high severity \\
\hline Structural Cracking Index & $\mathrm{SCl}$ & & 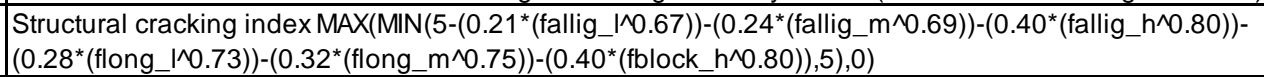 \\
\hline Environmental Cracking Index & $\mathrm{ECl}$ & & 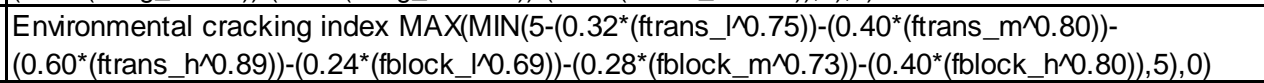 \\
\hline Rut Depth Index & RDI & & Rut depth index MAX(MIN(5-(6.65*(frut_mean^1.41)),5),0) \\
\hline Joint Condition Index & $\mathrm{JCl}$ & & 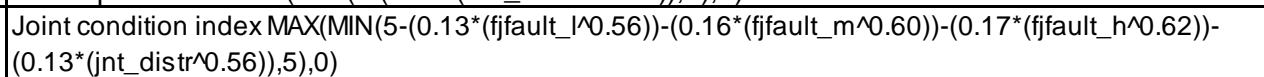 \\
\hline Concrete Slab Index & CSI & & 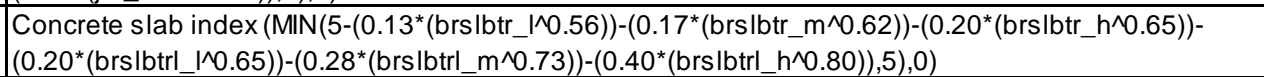 \\
\hline
\end{tabular}

Table 18: WVDOH PMS Legend for Distress Data as provided with Distress Summary 
Assumptions made during the comparison of ARAN and manual distress surveys on US 35 for the calculation of $\mathrm{CCl}$ and associated indices for asphalt section include:

- Data from Survey 1, 2, and 3 were used for comparison to WVDOH PMS data.

- Only data from the slow lane were used for comparison to WVDOH PMS data.

- The parameters for each severity of Alligator, Block, Transverse, and Longitudinal Cracking match identically between the two systems.

- The average of all the rut depth readings in BOTH wheel paths is RD.

- Only cracking within wheel paths is counted, i.e. joint cracking was counted in longitudinal cracking.

- The method for calculating percent of wheel path affected is the same as the method used to calculate percentage in Section 490, Special Provision.

Assumptions made during the comparison of ARAN and manual surveys on US 35 for the calculation of $\mathrm{CCl}$ and associated indices for concrete sections include:

- Data from Survey 1, 2, and 3 were used for comparison to WVDOH PMS data.

- Only data from the slow lane were used for comparison to WVDOH PMS data.

- The parameters for each severity of Faulting, Longitudinal Cracking, and Transverse Cracking match identically between the two systems.

- All transverse faults within the Section 490, Special Provision data were counted as + faults (there is no mention of direction of faults in surveys).

- General Joint Distress is the percentage of all transverse joints within the section that have any distresses (Faulting, Spalling, Cracking, etc. on transverse joints).

- Only Cracking within wheel paths is counted.

- The method for calculating percent of wheel path affected is the same as the method used to calculate percentage in Section 490, Special Provision.

Additional concerns regarding the comparison of WVDOH PMS data to PMS indices generated with the manual surface distress survey data include:

- Because the Section 490, Special Provision survey data does not include IRI data and there is no method of calculating IRI with the surveyed data, the Present Serviceability Index (PSI) cannot be determined.

- Because the PSI was the limiting factor in the determination of Composite Condition Index (CCI) in the WVDOH PMS data for both the HMA and PCC sections, comparing CCI values will be insignificant since PSI is not included in the Section 490, Special Provision data.

- In a few instances, if IRI within the PMS data were entered into the PSI equation, the resulting PSI slightly differed from the PSI provided by the PMS database. A summary of these differences 
is provided within Table 19. In this table, "PSI from equation" represents PSI when calculated using IRI and the PSI equation. "PSI given" represents the PSI directly from the WVDOH PMS database.

- Although the assumption was made to consider distress definitions and severity levels comparable between WVDOH PMS and Section 490, Special Provision, there were slight variations between the two in terms of distress and severity identification. A summary of the discrepancies is presented in Table 19.

- All other concerns regarding accuracy and consistency in using manually surveyed distress data to calculate PSR are still applicable.

\begin{tabular}{|c|c|c|c|}
\hline Mile Markers & $\begin{array}{c}\text { PSI from } \\
\text { equation }\end{array}$ & $\begin{array}{c}\text { PSI } \\
\text { Given }\end{array}$ \\
\hline HMA & $12.6-12.7$ & 2.74 & 3.00 \\
& $13.2-13.3$ & 4.01 & 4.01 \\
\hline & $13.8-13.9$ & 3.95 & 3.96 \\
\hline & $14.4-14.5$ & 3.83 & 3.83 \\
\hline & $14.6-14.7$ & 3.93 & 3.93 \\
\hline & $15.2-15.3$ & 3.29 & 3.33 \\
\hline & $15.4-15.5$ & 3.58 & 3.62 \\
\hline & $16.0-16.1$ & 3.91 & 3.91 \\
\hline & $16.4-16.5$ & 3.11 & 3.24 \\
\hline PCC & $9.1-9.2$ & 3.59 & 3.60 \\
\hline & $9.4-9.5$ & 3.75 & 3.76 \\
\hline & $10.4-10.5$ & 3.81 & 3.82 \\
\hline & $10.8-10.9$ & 3.87 & 3.87 \\
\hline & $11.4-11.5$ & 3.55 & 3.55 \\
\hline & $11.7-11.8$ & 3.85 & 3.85 \\
\hline & $12.2-12.3$ & 3.30 & 3.42 \\
\hline
\end{tabular}

Table 19: Variation between PSI calculated from IRI and PSI provided

In Table 20, parameters for distress identification for WVDOH PMS are abbreviated to PMS and for Section 490, Special Provision, the abbreviation S.P. is used. Indices pertaining to asphalt and concrete sections are in columns (a) and (b) respectively. Low, Medium, and High severities within distresses are labeled LS, MS, and HS respectively. Rows labeled "recording" indicated the method by which the distress is quantified. "\% wheelpath" means the percentage of wheelpath affected by the distress. Length is a linear measurement of the distress. Millimeter ranges indicate the range of crack widths which qualify as the given severity. "+ faults" means faults across a crack or joint where there is an increase in elevation from the leave to approach slab or panel. 
(a)

\begin{tabular}{|c|c|c|c|}
\hline HMA: & & & \\
\hline \multicolumn{4}{|c|}{ Structural Cracking Index (SCI) } \\
\hline & Allig. Cracks & PMS param. & SP param. \\
\hline & LS & $0-10 \mathrm{~mm}$ & \multirow{3}{*}{$\begin{array}{c}\text { Severity not } \\
\text { based on } \\
\text { crack width }\end{array}$} \\
\hline & MS & $10-18 \mathrm{~mm}$ & \\
\hline & HS & $>18 \mathrm{~mm}$ & \\
\hline & recording & $\%$ wheelpath & length \\
\hline & Long. Cracks & PMS param. & SP param. \\
\hline & LS & $0-10 \mathrm{~mm}$ & $0-6 \mathrm{~mm} \ldots$ \\
\hline & MS & $10-18 \mathrm{~mm}$ & $6-19 \mathrm{~mm} \ldots$ \\
\hline & HS & $>18 \mathrm{~mm}$ & $>19 \mathrm{~mm} . .$. \\
\hline & recording & $\%$ wheelpath & length \\
\hline & \multicolumn{3}{|c|}{$\begin{array}{l}\text { - PMS requires only distresses observed } \\
\text { withing the wheel paths which consist of 3- } \\
\text { foot wide areas starting } 2 \text { feet from either } \\
\text { edge of the lane. SP does not require } \\
\text { differentiation }\end{array}$} \\
\hline \multicolumn{4}{|c|}{ Environmental Cracking Index (ECI) } \\
\hline & Block Cracks & PMS param. & SP param. \\
\hline & LS & $0-10 \mathrm{~mm}$ & $0-6 \mathrm{~mm}$ \\
\hline & MS & $10-18 \mathrm{~mm}$ & $6-19 \mathrm{~mm} . .$. \\
\hline & HS & $>18 \mathrm{~mm}$ & $>19 \mathrm{~mm} . .$. \\
\hline & recording & $\%$ wheelpath & length \\
\hline & Trans. Cracks & PMS param. & SP param. \\
\hline & LS & $0-10 \mathrm{~mm}$ & $0-6 \mathrm{~mm} \ldots$ \\
\hline & MS & $10-18 \mathrm{~mm}$ & 6-19 mm... \\
\hline & HS & $>18 \mathrm{~mm}$ & $>19 \mathrm{~mm} \ldots$ \\
\hline & recording & $\%$ wheelpath & length \\
\hline \multicolumn{4}{|c|}{ Net Cracking Index ( $\mathrm{NCl})$} \\
\hline & \multicolumn{3}{|l|}{- still $\mathrm{SCl}+\mathrm{ECl}-5$} \\
\hline \multicolumn{4}{|c|}{ Rut Depth Index (RDI) } \\
\hline & Avg. Rut Depth & PMS param. & SP param. \\
\hline & recording & $\begin{array}{l}\text { continuous } \\
\text { average }\end{array}$ & \begin{tabular}{|c|} 
average of 5 \\
readings \\
taken at $100 \mathrm{ft}$ \\
intervals
\end{tabular} \\
\hline
\end{tabular}

(b)

PCC: Joint Conditioning Index (JCl)

\begin{tabular}{|c|c|c|}
\hline Faulting & PMS param. & SP param. \\
\hline LS & $0-6 \mathrm{~mm}$ & \multirow{3}{*}{$\begin{array}{c}\text { No } \\
\text { parameters } \\
\text { given }\end{array}$} \\
\hline MS & $6-12 \mathrm{~mm}$ & \\
\hline HS & $>12 \mathrm{~mm}$ & \\
\hline recording & only + faults & $+/$ - faults \\
\hline \multicolumn{3}{|c|}{ General Joint Distress } \\
\hline \multicolumn{3}{|c|}{ - not defined in PMS } \\
\hline \multicolumn{3}{|c|}{ - not included in SP } \\
\hline \multicolumn{3}{|l|}{ Slab Index } \\
\hline Trans. Cracks & PMS param. & SP param. \\
\hline LS & $0-12 \mathrm{~mm}$ & $0-3 \mathrm{~mm} . .$. \\
\hline MS & $12-25 \mathrm{~mm}$ & 3-13 $\mathrm{mm} . .$. \\
\hline $\mathrm{HS}$ & $>25 \mathrm{~mm}$ & $>13 \mathrm{~mm} \ldots$ \\
\hline Long. Cracks & PMS param. & SP param. \\
\hline LS & $0-12 \mathrm{~mm}$ & $0-3 \mathrm{~mm} . .$. \\
\hline MS & $12-25 \mathrm{~mm}$ & 3-6 mm... \\
\hline $\mathrm{HS}$ & $>25 \mathrm{~mm}$ & $>6 \mathrm{~mm} .$. \\
\hline
\end{tabular}

Table 20: Inconsistencies between WVDOH PMS and Section 490, Special Provision distress definition and severities

\subsection{Deflection Data Analysis}

For asphalt deflection tests, estimating a $\mathrm{SN}_{\text {eff }}$ was the primary method of comparing estimation methods and judging the structural quality of the pavement. Table 21 displays averaged $\mathrm{SN}_{\text {eff }}$ values calculated by all described methods and programs. Table 22 displays the identification of $\mathrm{SN}_{\text {eff }}$ values which were calculated using $M_{R^{*}}$ (highlighted values) as these estimated values consistently were higher than the WVDOH Design Directive's design structural number of approximately 6.7. 


\begin{tabular}{|c|c|c|c|c|c|}
\hline & $\begin{array}{l}S N_{\text {eff }} \text { as a function of } \\
E_{p} \text { as a function of } \\
M_{R} \text { from a } 3 \text { layer } \\
\text { MODULUS } 6.1 \\
\text { backcalculation }\end{array}$ & $\begin{array}{l}S N_{\text {eff }} \text { as a function of } \\
E_{p} \text { as a function of } \\
M_{R^{*}} \text { from a } 3 \text { layer } \\
\text { MODULUS } 6.1 \\
\text { backcalculation }\end{array}$ & $\begin{array}{l}\mathrm{SN}_{\text {eff }} \text { as a function of } \\
\mathrm{E}_{\mathrm{p}} \text { from a } 2 \text { layer } \\
\text { MODULUS } 6.1 \\
\text { backcalculation }\end{array}$ & $\begin{array}{l}\mathrm{SN}_{\text {eff }} \text { as a function of } \\
\mathrm{E}_{\mathrm{p}} \text { and } \mathrm{M}_{\mathrm{R}} \text { from a } 2 \\
\text { layer MODULUS } 6.1 \\
\text { backcalculation }\end{array}$ & $\begin{array}{l}S N_{\text {eff }} \text { as a function of } \\
E_{p} \text { and } M_{R^{*}} \text { from a } 2 \\
\text { layer MODULUS } 6.1 \\
\text { backcalculation }\end{array}$ \\
\hline Program & MODULUS 6.1 & MODULUS 6.1 & MODULUS 6.1 & MODULUS 6.1 & MODULUS 6.1 \\
\hline Backcalc using: & 3 Layer & 3 Layer & 2 Layer & 2 Layer & 2 Layer \\
\hline $\mathrm{E}_{\mathrm{p}}$ calculated with: & $M_{R}$ & $\mathrm{M}_{\mathrm{R}^{*}}$ & $\left(E_{p}\right.$ provided $)$ & $M_{R}$ & $\mathrm{M}_{\mathrm{R}^{*}}$ \\
\hline NB HMA Avg $\mathrm{SN}_{\mathrm{eff}}$ & 4.65 & 7.61 & 5.30 & 4.71 & 7.70 \\
\hline SB HMA Avg SN $\mathrm{Neff}$ & 4.19 & 6.52 & 4.76 & 4.24 & 6.61 \\
\hline All HMA Avg $\mathrm{SN}_{\mathrm{eff}}$ & 4.42 & 7.07 & 5.03 & 4.48 & 7.15 \\
\hline
\end{tabular}

\begin{tabular}{|c|c|c|c|c|c|}
\hline & $\begin{array}{l}\mathrm{SN}_{\text {eff }} \text { as provided by } \\
3 \text { layer MODTAG } \\
\text { backcalculation }\end{array}$ & $\begin{array}{l}\mathrm{SN}_{\text {eff }} \text { as a function of } \\
\mathrm{E}_{\mathrm{p}} \text { and } \mathrm{M}_{\mathrm{R}} \text { from a } 3 \\
\text { layer MODTAG } \\
\text { backcalculation }\end{array}$ & $\begin{array}{l}S N_{\text {eff }} \text { as a function of } \\
E_{p} \text { and } M_{R^{*}} \text { from a } 3 \\
\text { layer MODTAG } \\
\text { backcalculation }\end{array}$ & $\begin{array}{l}S N_{\text {eff }} \text { as a function of } \\
E_{p} \text { and } M_{R} \text { from a } 3 \\
\text { layer BACKFAA } \\
\text { backcalculation }\end{array}$ & $\begin{array}{l}\mathrm{SN}_{\text {eff }} \text { as a function of } \\
\mathrm{E}_{\mathrm{p}} \text { and } \mathrm{M}_{\mathrm{R}^{*}} \text { from a } 3 \\
\text { layer BACKFAA } \\
\text { backcalculation }\end{array}$ \\
\hline Program & MODTAG & MODTAG & MODTAG & BACKFAA & BACKFAA \\
\hline Backcalc using: & 3 Layer & 3 Layer & 3 Layer & 3 Layer & 3 Layer \\
\hline$E_{p}$ calculated with: & not calculated & $M_{R}$ & $\mathrm{M}_{\mathrm{R}^{*}}$ & $M_{R}$ & $\mathrm{M}_{\mathrm{R}^{*}}$ \\
\hline NB HMA Avg $\mathrm{SN}_{\text {eff }}$ & 4.31 & 4.90 & 7.88 & 5.25 & 8.94 \\
\hline SB HMA Avg $\mathrm{SN}_{\text {eff }}$ & 4.56 & 4.42 & 6.93 & 4.53 & 7.38 \\
\hline All HMA Avg SN $\mathrm{Sff}_{\text {ff }}$ & 4.44 & 4.66 & 7.41 & 4.89 & 8.16 \\
\hline
\end{tabular}

Table 21: Estimated $\mathrm{SN}_{\text {eff }}$ Summary

\begin{tabular}{|c|c|c|c|c|c|}
\hline & $\begin{array}{l}S N_{\text {eff }} \text { as a function of } \\
E_{p} \text { as a function of } \\
M_{R} \text { from a } 3 \text { layer } \\
\text { MODULUS } 6.1 \\
\text { backcalculation }\end{array}$ & $\begin{array}{l}\mathrm{SN}_{\text {eff }} \text { as a function of } \\
\mathrm{E}_{\mathrm{p}} \text { as a function of } \\
\mathrm{M}_{\mathrm{R}^{*}} \text { from a } 3 \text { layer } \\
\text { MODULUS } 6.1 \\
\text { backcalculation }\end{array}$ & $\begin{array}{l}S N_{\text {eff }} \text { as a function of } \\
E_{p} \text { from a } 2 \text { layer } \\
\text { MODULUS } 6.1 \\
\text { backcalculation }\end{array}$ & $\begin{array}{l}\mathrm{SN}_{\text {eff }} \text { as a function of } \\
\mathrm{E}_{\mathrm{p}} \text { and } \mathrm{M}_{\mathrm{R}} \text { from a } 2 \\
\text { layer MODULUS } 6.1 \\
\text { backcalculation }\end{array}$ & $\begin{array}{l}\mathrm{SN}_{\text {eff }} \text { as a function of } \\
\mathrm{E}_{\mathrm{p}} \text { and } \mathrm{M}_{\mathrm{R}^{*}} \text { from a } 2 \\
\text { layer MODULUS } 6.1 \\
\text { backcalculation }\end{array}$ \\
\hline Program & MODULUS 6.1 & MODULUS 6.1 & MODULUS 6.1 & MODULUS 6.1 & MODULUS 6.1 \\
\hline Backcalc using: & 3 Layer & 3 Layer & 2 Layer & 2 Layer & 2 Layer \\
\hline $\mathrm{E}_{\mathrm{p}}$ calculated with: & $M_{R}$ & $\mathrm{M}_{\mathrm{R}^{*}}$ & $\left(E_{p}\right.$ provided $)$ & $\mathrm{M}_{\mathrm{R}}$ & $\mathrm{M}_{\mathrm{R}^{*}}$ \\
\hline NB HMA Avg SN ${ }_{\text {eff }}$ & 4.65 & 7.61 & 5.30 & 4.71 & 7.70 \\
\hline SB HMA Avg SN ${ }_{\text {eff }}$ & 4.19 & 6.52 & 4.76 & 4.24 & 6.61 \\
\hline \multirow[t]{2}{*}{ All HMA Avg $\mathrm{SN}_{\mathrm{eff}}$} & 4.42 & 7.07 & 5.03 & 4.48 & 7.15 \\
\hline & $\begin{array}{l}\text { SN } \mathrm{eff}_{\text {as provided by }} \\
3 \text { layer MODTAG } \\
\text { backcalculation }\end{array}$ & $\begin{array}{l}\mathrm{SN}_{\text {eff }} \text { as a function of } \\
\mathrm{E}_{\mathrm{p}} \text { and } \mathrm{M}_{\mathrm{R}} \text { from a } 3 \\
\text { layer MODTAG } \\
\text { backcalculation }\end{array}$ & $\begin{array}{l}\mathrm{SN}_{\text {eff }} \text { as a function of } \\
\mathrm{E}_{\mathrm{p}} \text { and } \mathrm{M}_{\mathrm{R}^{*}} \text { from a } 3 \\
\text { layer MODTAG } \\
\text { backcalculation }\end{array}$ & $\begin{array}{l}\mathrm{SN}_{\text {eff }} \text { as a function of } \\
\mathrm{E}_{\mathrm{p}} \text { and } \mathrm{M}_{\mathrm{R}} \text { from a } 3 \\
\text { layer BACKFAA } \\
\text { backcalculation }\end{array}$ & $\begin{array}{l}\mathrm{SN}_{\text {eff }} \text { as a function of } \\
\mathrm{E}_{\mathrm{p}} \text { and } \mathrm{M}_{\mathrm{R}^{*}} \text { from a } 3 \\
\text { layer BACKFAA } \\
\text { backcalculation }\end{array}$ \\
\hline Program & MODTAG & MODTAG & MODTAG & BACKFAA & BACKFAA \\
\hline Backcalc using: & 3 Layer & 3 Layer & 3 Layer & 3 Layer & 3 Layer \\
\hline $\mathrm{E}_{\mathrm{p}}$ calculated with: & not calculated & $M_{R}$ & $\mathrm{M}_{\mathrm{R}^{*}}$ & $M_{R}$ & $M_{R^{*}}$ \\
\hline NB HMA Avg SN $\mathrm{eff}$ & 4.31 & 4.90 & 7.88 & 5.25 & 8.94 \\
\hline SB HMA Avg SN $\mathrm{eff}$ & 4.56 & 4.42 & 6.93 & 4.53 & 7.38 \\
\hline All HMA Avg SN $\mathrm{eff}_{\mathrm{e}}$ & 4.44 & 4.66 & 7.41 & 4.89 & 8.16 \\
\hline
\end{tabular}

Table 22: Estimated $S_{\text {eff }}$ Identification of Values Generated using $M_{R^{*}}$ 
Table 23 and Table 24 introduce the variable of pavements constructed on cuts and fills.

Because US 35 is constructed in a location which required cut and fill construction, the potential of varying bedrock depths and subgrade conditions was identified as a possible factor in the variation of deflection data.

\begin{tabular}{|c|c|c|c|c|c|c|c|c|}
\hline $\begin{array}{c}\text { Dir. } \\
\text { (North/ } \\
\text { South) }\end{array}$ & $\begin{array}{l}\text { Mile } \\
\text { Post } \\
(\mathrm{mi})\end{array}$ & $\begin{array}{c}\text { CUT/ } \\
\text { FILL }\end{array}$ & \begin{tabular}{|l|}
$\mathrm{SN}_{\text {eff }}$ as a \\
function of $E_{p}$ \\
as a function of \\
$\mathrm{M}_{\mathrm{R}}$ from a 3 \\
layer \\
MODULUS 6.1 \\
backcalculation \\
(Avg of all \\
drops)
\end{tabular} & $\begin{array}{l}\mathrm{SN}_{\text {eff }} \text { as a } \\
\text { function of } \mathrm{E}_{\mathrm{p}} \\
\text { from a } 2 \text { layer } \\
\text { MODULUS } 6.1 \\
\text { backcalculation } \\
\text { (Avg of all } \\
\text { drops) }\end{array}$ & $\begin{array}{l}S_{\text {eff }} \text { as a } \\
\text { function of } E_{p} \\
\text { and } M_{R} \text { from a } 2 \\
\text { layer } \\
\text { MODULUS } 6.1 \\
\text { backcalculation } \\
\text { (Avg of all } \\
\text { drops) }\end{array}$ & \begin{tabular}{|l|}
$\mathrm{SN}_{\text {eff }}$ as \\
provided by 3 \\
layer MODTAG \\
backcalculation \\
(Avg of all \\
drops)
\end{tabular} & $\begin{array}{l}\mathrm{SN}_{\text {eff }} \text { as a } \\
\text { function of } \mathrm{E}_{\mathrm{p}} \\
\text { and } \mathrm{M}_{\mathrm{R}} \text { from a } 3 \\
\text { layer MODTAG } \\
\text { backcalculation } \\
\text { (Avg of all } \\
\text { drops) }\end{array}$ & \begin{tabular}{|l|}
$\mathrm{SN}_{\text {eff }}$ as a \\
function of $E_{p}$ \\
and $M_{R}$ from a 3 \\
layer BACKFAA \\
backcalculation \\
(12th drop \\
only)
\end{tabular} \\
\hline
\end{tabular}

\begin{tabular}{|c|c|c|c|c|c|c|c|c|}
\hline$N$ & 16.2 & $\mathrm{~F}$ & 5.04 & 5.70 & 5.10 & 4.58 & 5.04 & 5.35 \\
\hline$N$ & 16.1 & $\mathrm{~F}$ & 4.55 & 5.11 & 4.61 & 4.17 & 4.80 & 5.10 \\
\hline$N$ & 15.8 & $\mathrm{~F}$ & 4.32 & 5.01 & 4.34 & 3.77 & 4.82 & 4.95 \\
\hline$N$ & 15.7 & $\mathrm{~F}$ & 5.17 & 6.05 & 5.31 & 4.67 & 5.40 & 5.71 \\
\hline$N$ & 15.2 & $\mathrm{~F}$ & 4.24 & 4.78 & 4.26 & 3.85 & 4.40 & 5.10 \\
\hline$N$ & 15.1 & C & 4.33 & 4.95 & 4.36 & 3.81 & 4.60 & 5.40 \\
\hline$N$ & 15.0 & C & 4.55 & 5.10 & 4.59 & 4.11 & 4.91 & 5.23 \\
\hline$N$ & 14.9 & $\mathrm{~F}$ & 5.12 & 5.88 & 5.28 & 5.18 & 5.61 & 6.13 \\
\hline$N$ & 14.4 & C & 5.06 & 5.78 & 5.15 & 4.87 & 5.46 & 5.86 \\
\hline $\mathrm{N}$ & 14.3 & C & 4.76 & 5.49 & 4.80 & 4.17 & 4.95 & 5.26 \\
\hline$N$ & 14.2 & C & 5.20 & 5.93 & 5.36 & 5.08 & 5.46 & 5.53 \\
\hline$N$ & 14.1 & $\mathrm{~F}$ & 4.24 & 4.80 & 4.27 & 3.87 & 4.56 & 4.63 \\
\hline$N$ & 13.9 & C & 4.34 & 4.94 & 4.36 & 3.93 & 4.56 & 4.65 \\
\hline$N$ & 13.8 & $\mathrm{~F}$ & 4.70 & 5.35 & 4.73 & 4.32 & 4.85 & 5.05 \\
\hline$N$ & 13.3 & $\mathrm{~F}$ & 4.28 & 4.89 & 4.31 & 4.03 & 4.34 & 5.09 \\
\hline$N$ & 13.2 & $\mathrm{~F}$ & 4.78 & 5.49 & 4.83 & 4.47 & 4.89 & 5.19 \\
\hline$N$ & 12.7 & $\mathrm{~F}$ & 4.68 & 5.25 & 4.74 & 4.59 & 5.02 & 5.14 \\
\hline $\mathrm{N}$ & 12.6 & C & 4.40 & 4.97 & 4.42 & 4.06 & 4.48 & 5.10 \\
\hline$S$ & 12.6 & C & 4.08 & 4.58 & 4.09 & 4.58 & 4.43 & 4.58 \\
\hline$S$ & 12.7 & C & 4.32 & 4.89 & 4.38 & 4.75 & 4.54 & 4.60 \\
\hline $\mathrm{S}$ & 13.2 & C & 4.83 & 5.53 & 4.84 & 5.53 & 4.90 & 4.93 \\
\hline $\mathrm{S}$ & 13.3 & $\mathrm{~F}$ & 3.97 & 4.46 & 3.98 & 4.21 & 4.31 & 4.40 \\
\hline$S$ & 13.7 & $\mathrm{~F}$ & 4.21 & 4.74 & 4.29 & 4.74 & 4.59 & 4.79 \\
\hline $\mathrm{s}$ & 13.8 & C & 4.08 & 4.59 & 4.17 & 4.42 & 4.23 & 4.49 \\
\hline $\mathrm{S}$ & 14.3 & C & 4.26 & 4.89 & 4.28 & 4.42 & 4.40 & 4.35 \\
\hline $\mathrm{S}$ & 14.4 & C & 3.66 & 4.12 & 3.71 & 3.84 & 3.88 & 3.84 \\
\hline$S$ & 14.5 & $\mathrm{~F}$ & 3.87 & 4.37 & 3.88 & 4.05 & 4.06 & 4.16 \\
\hline$S$ & 14.6 & C & 4.15 & 4.68 & 4.26 & 4.67 & 4.43 & 4.55 \\
\hline $\mathrm{s}$ & 15.1 & $\mathrm{~F}$ & 3.47 & 4.00 & 3.56 & 3.73 & 3.74 & 3.87 \\
\hline $\mathrm{S}$ & 15.2 & C & 3.89 & 4.29 & 3.92 & 3.80 & 3.92 & 3.93 \\
\hline S & 15.3 & C & 3.87 & 4.35 & 3.92 & 4.03 & 4.10 & 4.22 \\
\hline S & 15.4 & $\mathrm{~F}$ & 4.19 & 4.72 & 4.30 & 4.88 & 4.44 & 4.67 \\
\hline$S$ & 15.9 & C & 4.62 & 5.30 & 4.64 & 5.24 & 4.76 & 4.73 \\
\hline$S$ & 16.0 & $\mathrm{~F}$ & 4.77 & 5.60 & 4.79 & 5.11 & 4.83 & 5.06 \\
\hline S & 16.4 & C & 4.55 & 5.23 & 4.56 & 4.81 & 4.89 & 4.84 \\
\hline $\mathrm{S}$ & 16.5 & $\mathrm{~F}$ & 4.66 & 5.39 & 4.67 & 5.37 & 5.15 & 5.60 \\
\hline N Avg & & & 4.65 & 5.30 & 4.71 & 4.31 & 4.90 & 5.25 \\
\hline S Avg & & & 4.19 & 4.76 & 4.24 & 4.56 & 4.42 & 4.53 \\
\hline Avg & & & 4.42 & 5.03 & 4.48 & 4.44 & 4.66 & 4.89 \\
\hline
\end{tabular}

Table 23: Summary of Asphalt Section SNeff Values at Each Test Location with Addition of Cut/Fill Information 


\begin{tabular}{|c|c|c|c|c|c|c|c|c|}
\hline $\begin{array}{c}\text { Dir. } \\
\text { (North/ } \\
\text { South) }\end{array}$ & $\begin{array}{l}\text { Mile } \\
\text { Post } \\
\text { (mi) }\end{array}$ & $\begin{array}{c}\text { CUT/ } \\
\text { FILL }\end{array}$ & $\begin{array}{l}S N_{\text {eff }} \text { as a } \\
\text { function of } E_{p} \\
\text { as a function of } \\
M_{R} \text { from a } 3 \\
\text { layer } \\
\text { MODULUS } 6.1 \\
\text { backcalculation } \\
\text { (Avg of all } \\
\text { drops) }\end{array}$ & $\begin{array}{l}\mathrm{SN}_{\text {eff }} \text { as a } \\
\text { function of } E_{p} \\
\text { from a } 2 \text { layer } \\
\text { MODULUS } 6.1 \\
\text { backcalculation } \\
\text { (Avg of all } \\
\text { drops) }\end{array}$ & $\begin{array}{l}\mathrm{SN}_{\text {eff }} \text { as a } \\
\text { function of } \mathrm{E}_{\mathrm{p}} \\
\text { and } \mathrm{M}_{\mathrm{R}} \text { from a } 2 \\
\text { layer } \\
\text { MODULUS } 6.1 \\
\text { backcalculation } \\
\text { (Avg of all } \\
\text { drops) }\end{array}$ & $\begin{array}{l}\mathrm{SN}_{\text {eff }} \text { as } \\
\text { provided by } 3 \\
\text { layer MODTAG } \\
\text { backcalculation } \\
\text { (Avg of all } \\
\text { drops) }\end{array}$ & $\begin{array}{l}S_{\text {eff }} \text { as a } \\
\text { function of } E_{p} \\
\text { and } M_{R} \text { from a } 3 \\
\text { layer MODTAG } \\
\text { backcalculation } \\
\text { (Avg of all } \\
\text { drops) }\end{array}$ & $\begin{array}{l}\mathrm{SN}_{\text {eff }} \text { as a } \\
\text { function of } \mathrm{E}_{\mathrm{p}} \\
\text { and } \mathrm{M}_{\mathrm{R}} \text { from a } 3 \\
\text { layer BACKFAA } \\
\text { backcalculation } \\
\text { (12th drop } \\
\text { only) }\end{array}$ \\
\hline
\end{tabular}

\begin{tabular}{|c|c|c|c|c|c|c|c|c|}
\hline $\mathrm{N}$ & 15.1 & C & 4.33 & 4.95 & 4.36 & 3.81 & 4.60 & 5.40 \\
\hline $\mathrm{N}$ & 15.0 & C & 4.55 & 5.10 & 4.59 & 4.11 & 4.91 & 5.23 \\
\hline $\mathrm{N}$ & 14.4 & C & 5.06 & 5.78 & 5.15 & 4.87 & 5.46 & 5.86 \\
\hline $\mathrm{N}$ & 14.3 & C & 4.76 & 5.49 & 4.80 & 4.17 & 4.95 & 5.26 \\
\hline $\mathrm{N}$ & 14.2 & C & 5.20 & 5.93 & 5.36 & 5.08 & 5.46 & 5.53 \\
\hline $\mathrm{N}$ & 13.9 & C & 4.34 & 4.94 & 4.36 & 3.93 & 4.56 & 4.65 \\
\hline $\mathrm{N}$ & 12.6 & $C$ & 4.40 & 4.97 & 4.42 & 4.06 & 4.48 & 5.10 \\
\hline$S$ & 12.6 & C & 4.08 & 4.58 & 4.09 & 4.58 & 4.43 & 4.58 \\
\hline$S$ & 12.7 & C & 4.32 & 4.89 & 4.38 & 4.75 & 4.54 & 4.60 \\
\hline$S$ & 13.2 & C & 4.83 & 5.53 & 4.84 & 5.53 & 4.90 & 4.93 \\
\hline$S$ & 13.8 & C & 4.08 & 4.59 & 4.17 & 4.42 & 4.23 & 4.49 \\
\hline$S$ & 14.3 & C & 4.26 & 4.89 & 4.28 & 4.42 & 4.40 & 4.35 \\
\hline$S$ & 14.4 & C & 3.66 & 4.12 & 3.71 & 3.84 & 3.88 & 3.84 \\
\hline$S$ & 14.6 & C & 4.15 & 4.68 & 4.26 & 4.67 & 4.43 & 4.55 \\
\hline$S$ & 15.2 & C & 3.89 & 4.29 & 3.92 & 3.80 & 3.92 & 3.93 \\
\hline$S$ & 15.3 & C & 3.87 & 4.35 & 3.92 & 4.03 & 4.10 & 4.22 \\
\hline$S$ & 15.9 & C & 4.62 & 5.30 & 4.64 & 5.24 & 4.76 & 4.73 \\
\hline $\mathrm{S}$ & 16.4 & C & 4.55 & 5.23 & 4.56 & 4.81 & 4.89 & 4.84 \\
\hline $\mathrm{N}$ & 16.2 & $\mathrm{~F}$ & 5.04 & 5.70 & 5.10 & 4.58 & 5.04 & 5.35 \\
\hline $\mathrm{N}$ & 16.1 & $\mathrm{~F}$ & 4.55 & 5.11 & 4.61 & 4.17 & 4.80 & 5.10 \\
\hline $\mathrm{N}$ & 15.8 & $\mathrm{~F}$ & 4.32 & 5.01 & 4.34 & 3.77 & 4.82 & 4.95 \\
\hline $\mathrm{N}$ & 15.7 & $\mathrm{~F}$ & 5.17 & 6.05 & 5.31 & 4.67 & 5.40 & 5.71 \\
\hline $\mathrm{N}$ & 15.2 & $\mathrm{~F}$ & 4.24 & 4.78 & 4.26 & 3.85 & 4.40 & 5.10 \\
\hline $\mathrm{N}$ & 14.9 & $\mathrm{~F}$ & 5.12 & 5.88 & 5.28 & 5.18 & 5.61 & 6.13 \\
\hline $\mathrm{N}$ & 14.1 & $\mathrm{~F}$ & 4.24 & 4.80 & 4.27 & 3.87 & 4.56 & 4.63 \\
\hline $\mathrm{N}$ & 13.8 & $\mathrm{~F}$ & 4.70 & 5.35 & 4.73 & 4.32 & 4.85 & 5.05 \\
\hline $\mathrm{N}$ & 13.3 & $\mathrm{~F}$ & 4.28 & 4.89 & 4.31 & 4.03 & 4.34 & 5.09 \\
\hline $\mathrm{N}$ & 13.2 & $\mathrm{~F}$ & 4.78 & 5.49 & 4.83 & 4.47 & 4.89 & 5.19 \\
\hline $\mathrm{N}$ & 12.7 & $\mathrm{~F}$ & 4.68 & 5.25 & 4.74 & 4.59 & 5.02 & 5.14 \\
\hline$S$ & 13.3 & $\mathrm{~F}$ & 3.97 & 4.46 & 3.98 & 4.21 & 4.31 & 4.40 \\
\hline$S$ & 13.7 & $\mathrm{~F}$ & 4.21 & 4.74 & 4.29 & 4.74 & 4.59 & 4.79 \\
\hline$S$ & 14.5 & $\mathrm{~F}$ & 3.87 & 4.37 & 3.88 & 4.05 & 4.06 & 4.16 \\
\hline$S$ & 15.1 & $F$ & 3.47 & 4.00 & 3.56 & 3.73 & 3.74 & 3.87 \\
\hline$S$ & 15.4 & $\mathrm{~F}$ & 4.19 & 4.72 & 4.30 & 4.88 & 4.44 & 4.67 \\
\hline$S$ & 16.0 & $\mathrm{~F}$ & 4.77 & 5.60 & 4.79 & 5.11 & 4.83 & 5.06 \\
\hline $\mathrm{S}$ & 16.5 & $\mathrm{~F}$ & 4.66 & 5.39 & 4.67 & 5.37 & 5.15 & 5.60 \\
\hline \multicolumn{2}{|c|}{ Avg } & CUT & 4.39 & 4.98 & 4.43 & 4.45 & 4.61 & 4.78 \\
\hline \multicolumn{2}{|c|}{ Avg } & FILL & 4.46 & 5.09 & 4.52 & 4.42 & 4.71 & 5.00 \\
\hline
\end{tabular}

Table 24: Reorganization of Table 23 by Cuts and Fills 
Table 25 shows the BAKFAA backcalculated moduli values for the surface, base, and subgrade for all concrete section tests which were eligible for analysis. The subgrade modulus values will be compared to those backcalculated from asphalt section deflection data.

\begin{tabular}{|c|c|c|c|c|c|}
\hline $\begin{array}{c}\text { Dir. } \\
\text { (North/ } \\
\text { South) }\end{array}$ & $\begin{array}{c}\text { Mile Post } \\
(\mathrm{mi})\end{array}$ & $\begin{array}{c}\text { Mile Post } \\
(\mathrm{ft})\end{array}$ & $\begin{array}{c}\text { Surface } \\
\text { Modulus } \\
(\mathrm{ksi})\end{array}$ & $\begin{array}{c}\text { Base } \\
\text { Modulus } \\
(\mathrm{ksi})\end{array}$ & $\begin{array}{c}\text { Subgrade } \\
\text { Modulus } \\
(\mathrm{ksi})\end{array}$ \\
\hline
\end{tabular}

\begin{tabular}{|c|c|c|c|c|c|}
\hline $\mathrm{N}$ & 10.9 & 57705 & 9491 & 217 & 29 \\
\hline $\mathrm{N}$ & 10.9 & 57705 & 12339 & 255 & 26 \\
\hline $\mathrm{N}$ & 10.9 & 57705 & 12384 & 255 & 26 \\
\hline $\mathrm{N}$ & 10.8 & 57182 & 4853 & 234 & 28 \\
\hline $\mathrm{N}$ & 10.8 & 57182 & 3999 & 147 & 33 \\
\hline $\mathrm{N}$ & 10.5 & 55593 & 8741 & 130 & 13 \\
\hline $\mathrm{N}$ & 10.5 & 55593 & 7429 & 149 & 16 \\
\hline $\mathrm{N}$ & 10.4 & 55076 & 12458 & 258 & 26 \\
\hline $\mathrm{N}$ & 10.4 & 55076 & 12736 & 263 & 26 \\
\hline $\mathrm{N}$ & 10.4 & 55076 & 9256 & 293 & 32 \\
\hline $\mathrm{N}$ & 9.5 & 50382 & 12948 & 268 & 27 \\
\hline $\mathrm{N}$ & 9.5 & 50382 & 8791 & 276 & 33 \\
\hline $\mathrm{N}$ & 9.5 & 50382 & 8656 & 269 & 31 \\
\hline$S$ & 9.2 & 48576 & 22110 & 349 & 28 \\
\hline$S$ & 9.2 & 48576 & 17073 & 359 & 35 \\
\hline$S$ & 9.2 & 48576 & 28114 & 365 & 25 \\
\hline$S$ & 9.5 & 50149 & 16089 & 338 & 33 \\
\hline$S$ & 9.5 & 50149 & 15407 & 321 & 32 \\
\hline$S$ & 9.5 & 50149 & 15952 & 334 & 33 \\
\hline$S$ & 11.4 & 60076 & 7545 & 111 & 19 \\
\hline$S$ & 11.4 & 60076 & 7537 & 111 & 19 \\
\hline$S$ & 11.4 & 60076 & 7051 & 165 & 18 \\
\hline$S$ & 11.7 & 61644 & 7125 & 251 & 28 \\
\hline$S$ & 11.7 & 61644 & 7012 & 203 & 28 \\
\hline$S$ & 11.7 & 61644 & 7048 & 193 & 27 \\
\hline$S$ & 11.8 & 62172 & 8711 & 217 & 25 \\
\hline$S$ & 11.8 & 62172 & 11572 & 240 & 24 \\
\hline$S$ & 11.8 & 62172 & 11135 & 230 & 24 \\
\hline$S$ & 12.2 & 64506 & 4165 & 68 & 26 \\
\hline$S$ & 12.2 & 64506 & 8781 & 179 & 19 \\
\hline$S$ & 12.2 & 64506 & 3814 & 101 & 26 \\
\hline$S$ & 12.3 & 65039 & 10434 & 230 & 19 \\
\hline$S$ & 12.3 & 65039 & 8921 & 182 & 19 \\
\hline$S$ & 12.3 & 65039 & 11804 & 236 & 18 \\
\hline N Avg & & & 9545 & 232 & 26.6 \\
\hline S Avg & & & 11305 & 228 & 25.0 \\
\hline Avg & & & 10425 & 230 & 25.8 \\
\hline
\end{tabular}

Table 25: BAKFAA Moduli Values for Eligible Concrete Drops 


\section{Chapter 5. Results}

\subsection{Pavement Surface Rating}

\subsubsection{US 35 PSR Original 2012 Spec. Calculation}

Calculated PSR values for northbound asphalt and concrete sections following the initial analysis in July 2013 are summarized within Table 26 and Table 27 respectively. Calculated PSR values for southbound asphalt and concrete sections are summarized within Table 28 and Table 29 respectively. The 2012 Special Provision PSR calculation procedure was used for this analysis as it served as the starting point for this research.

\begin{tabular}{|l|ccc|ccc|}
\cline { 2 - 6 } \multicolumn{1}{c|}{} & $\begin{array}{c}\text { Slow Lane } \\
\text { Mile Marker }\end{array}$ & $\begin{array}{c}\text { Slow Lane } \\
\text { Survey 1 }\end{array}$ & $\begin{array}{c}\text { Slow Lane } \\
\text { Survey 2 }\end{array}$ & $\begin{array}{c}\text { Fast Lane } \\
\text { Survey } 3\end{array}$ & $\begin{array}{c}\text { Fast Lane } \\
\text { Survey 2 }\end{array}$ & $\begin{array}{c}\text { Fast Lane } \\
\text { Survey 3 }\end{array}$ \\
\hline $12.6-12.7$ & 86.46 & 86.46 & 69.75 & 91.37 & 91.37 & 91.37 \\
$13.2-13.3$ & 88.30 & 88.58 & 82.04 & 91.37 & 91.37 & 91.37 \\
$13.8-13.9$ & 90.99 & 90.99 & 88.97 & 91.37 & 91.37 & 91.37 \\
$14.4-14.5$ & 90.22 & 90.22 & 80.40 & 91.37 & 91.37 & 91.37 \\
$14.6-14.7$ & 91.37 & 91.37 & 91.37 & 91.37 & 91.37 & 91.37 \\
$15.2-15.3$ & 86.68 & 86.68 & 72.25 & 90.90 & 90.44 & 90.21 \\
$15.4-15.5$ & 89.56 & 87.57 & 78.69 & 89.75 & 89.29 & 89.06 \\
$16.0-16.1$ & 87.61 & 86.52 & 83.73 & 89.09 & 89.09 & 89.03 \\
$16.4-16.5$ & 88.61 & 88.61 & 86.90 & 91.37 & 91.37 & 91.37 \\
\hline Average & 88.87 & 88.55 & 81.57 & 90.88 & 90.78 & 90.72 \\
\hline
\end{tabular}

Table 26: Initial PSR Calculation for Northbound Asphalt Section

\begin{tabular}{|c|c|c|c|c|c|c|}
\hline Mile Marker & $\begin{array}{c}\text { Slow Lane } \\
\text { Survey } 1\end{array}$ & $\begin{array}{l}\text { Slow Lane } \\
\text { Survey } 2\end{array}$ & $\begin{array}{l}\text { Slow Lane } \\
\text { Survey } 3\end{array}$ & $\begin{array}{c}\text { Fast Lane } \\
\text { Survey } 1\end{array}$ & $\begin{array}{l}\text { Fast Lane } \\
\text { Survey } 2\end{array}$ & $\begin{array}{c}\text { Fast Lane } \\
\text { Survey } 3\end{array}$ \\
\hline $9.1-9.2$ & 85.35 & 79.01 & 78.30 & 99.97 & 99.97 & 98.06 \\
\hline $9.4-9.5$ & 99.97 & 66.68 & 64.99 & 99.97 & 59.39 & 59.39 \\
\hline $10.4-10.5$ & 90.78 & 76.32 & 68.41 & 98.06 & 89.62 & 69.75 \\
\hline $10.8-10.9$ & 95.57 & 65.40 & 57.88 & 98.69 & 60.94 & 62.13 \\
\hline $11.4-11.5$ & 99.97 & 98.06 & 92.55 & 98.04 & 94.33 & 89.03 \\
\hline $11.7-11.8$ & 98.06 & 90.20 & 92.55 & 96.19 & 82.44 & 79.32 \\
\hline $12.2-12.3$ & 93.15 & 91.37 & 91.37 & 94.96 & 93.15 & 89.62 \\
\hline Average & 94.69 & 81.01 & 78.01 & 97.98 & 82.83 & 78.19 \\
\hline
\end{tabular}

Table 27: Initial PSR Calculation for Northbound Concrete Section 


\begin{tabular}{|l|ccc|ccc|}
\cline { 2 - 7 } \multicolumn{1}{c|}{ Mile Marker } & $\begin{array}{c}\text { Slow Lane } \\
\text { Survey 1 }\end{array}$ & $\begin{array}{c}\text { Slow Lane } \\
\text { Survey 2 }\end{array}$ & $\begin{array}{c}\text { Slow Lane } \\
\text { Survey 3 }\end{array}$ & $\begin{array}{c}\text { Fast Lane } \\
\text { Survey 1 }\end{array}$ & $\begin{array}{c}\text { Fast Lane } \\
\text { Survey 2 }\end{array}$ & $\begin{array}{c}\text { Fast Lane } \\
\text { Survey 3 }\end{array}$ \\
\hline $12.7-12.6$ & 81.06 & 76.76 & 76.76 & 91.37 & 79.10 & 79.10 \\
$13.3-13.2$ & 87.17 & 86.55 & 73.37 & 91.37 & 91.37 & 91.37 \\
$13.9-13.8$ & 87.84 & 87.84 & 87.84 & 69.75 & 69.75 & 69.75 \\
$14.5-14.4$ & 90.58 & 90.58 & 87.97 & 91.37 & 91.37 & 91.37 \\
$14.7-14.6$ & 99.97 & 99.97 & 83.33 & 83.50 & 83.50 & 83.50 \\
$15.3-15.2$ & 76.04 & 73.28 & 69.75 & 84.84 & 84.84 & 84.84 \\
$15.5-15.4$ & 84.27 & 84.27 & 84.27 & 91.37 & 91.37 & 91.37 \\
$16.1-16.0$ & 89.98 & 88.56 & 85.53 & 91.37 & 91.21 & 91.21 \\
$16.5-16.4$ & 91.37 & 90.51 & 78.51 & 91.37 & 91.37 & 91.37 \\
\hline Average & 87.59 & 86.48 & 80.81 & 87.36 & 85.98 & 85.98 \\
\hline
\end{tabular}

Table 28: Initial PSR Calculation for Southbound Asphalt Section

\begin{tabular}{|l|ccc|ccc|}
\cline { 2 - 6 } \multicolumn{1}{c|}{ Mile Marker } & $\begin{array}{c}\text { Slow Lane } \\
\text { Survey 1 }\end{array}$ & $\begin{array}{c}\text { Slow Lane } \\
\text { Survey 2 }\end{array}$ & $\begin{array}{c}\text { Slow Lane } \\
\text { Survey 3 }\end{array}$ & $\begin{array}{c}\text { Fast Lane } \\
\text { Survey 1 }\end{array}$ & $\begin{array}{c}\text { Fast Lane } \\
\text { Survey 2 }\end{array}$ & $\begin{array}{c}\text { Fast Lane } \\
\text { Survey 3 }\end{array}$ \\
\cline { 1 - 6 } $9.3-9.2$ & 53.93 & 51.10 & 47.67 & 90.08 & 75.73 & 62.29 \\
$9.5-9.4$ & 62.29 & 53.59 & 51.56 & 59.55 & 55.69 & 55.69 \\
$10.5-10.4$ & 79.32 & 75.83 & 75.83 & 91.60 & 81.59 & 81.59 \\
$10.9-10.8$ & 77.70 & 77.30 & 75.83 & 86.67 & 81.59 & 77.01 \\
$11.5-11.4$ & 81.38 & 80.34 & 80.34 & 85.13 & 78.81 & 78.81 \\
$11.8-11.7$ & 81.41 & 76.45 & 75.67 & 92.25 & 88.64 & 83.34 \\
$12.35-12.25$ & 77.30 & 74.19 & 73.81 & 83.29 & 75.34 & 73.81 \\
\hline Average & 73.33 & 69.83 & 68.67 & 84.08 & 76.77 & 73.22 \\
\hline
\end{tabular}

Table 29: Initial PSR Calculation for Southbound Concrete Section 


\subsubsection{Comparison and Correlation of PSR and PCI}

The final version of the PSR calculation method was used to analyze the 77 asphalt and concrete surface distress scenarios. Figure 14 and Figure 15 present the comparisons. These plots are the end result of all included distresses and any changes to their definitions or weight factors. Both plots include a linear trendline, an equation for the trendline, an $\mathrm{R}^{2}$ value, and a standard error of the data. Figure 16 contains all 77 scenarios as well as both trendlines plotted together. Figure 17 is the same graph as Figure 16, this time with a single trendline for all 77 data points. Individual asphalt and concrete data points still are represented by separate markers, but the trendline was generated by the combination of all points.

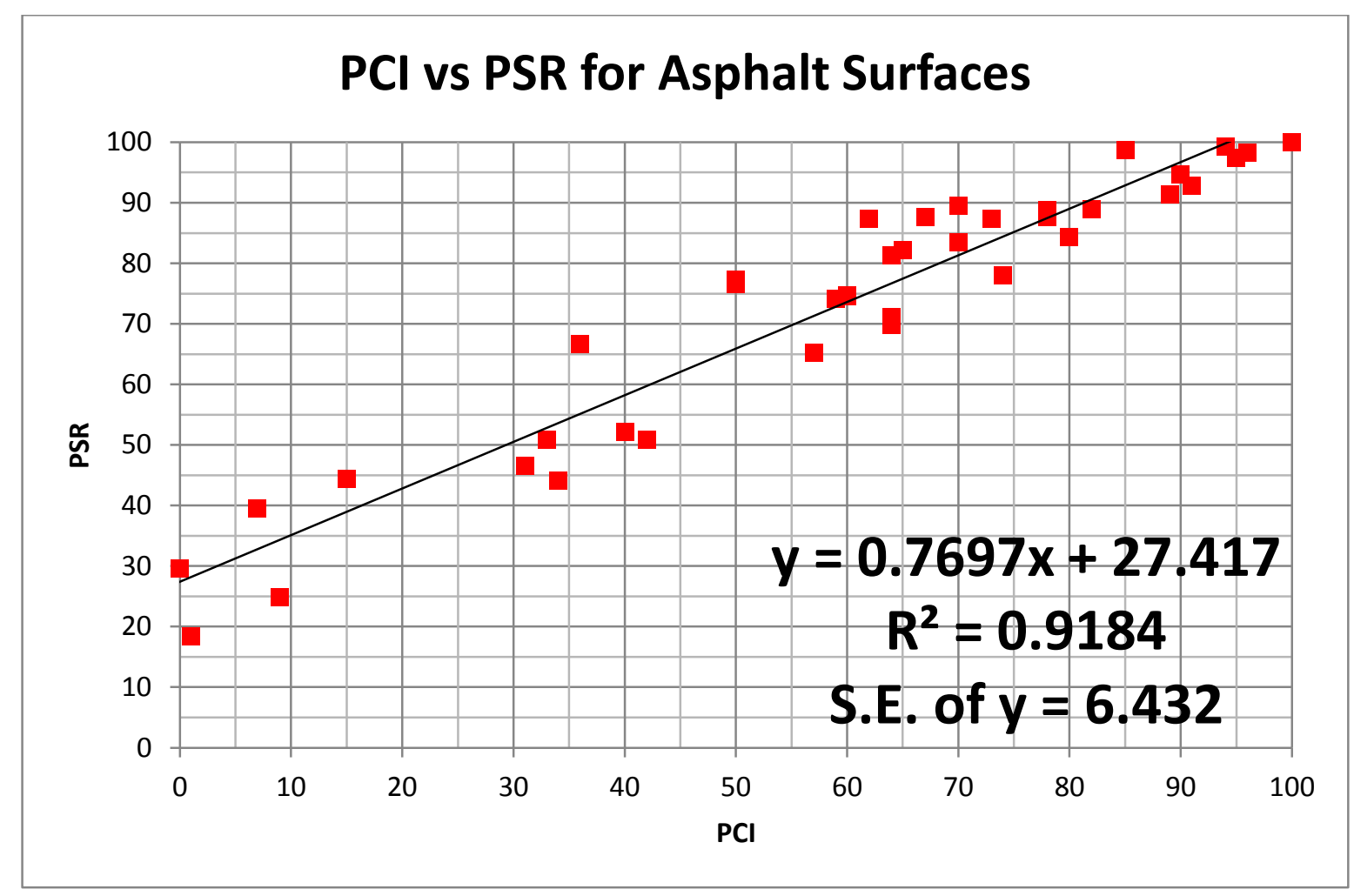

Figure 14: Final PCI vs. PSR Correlation for Asphalt Surfaces 


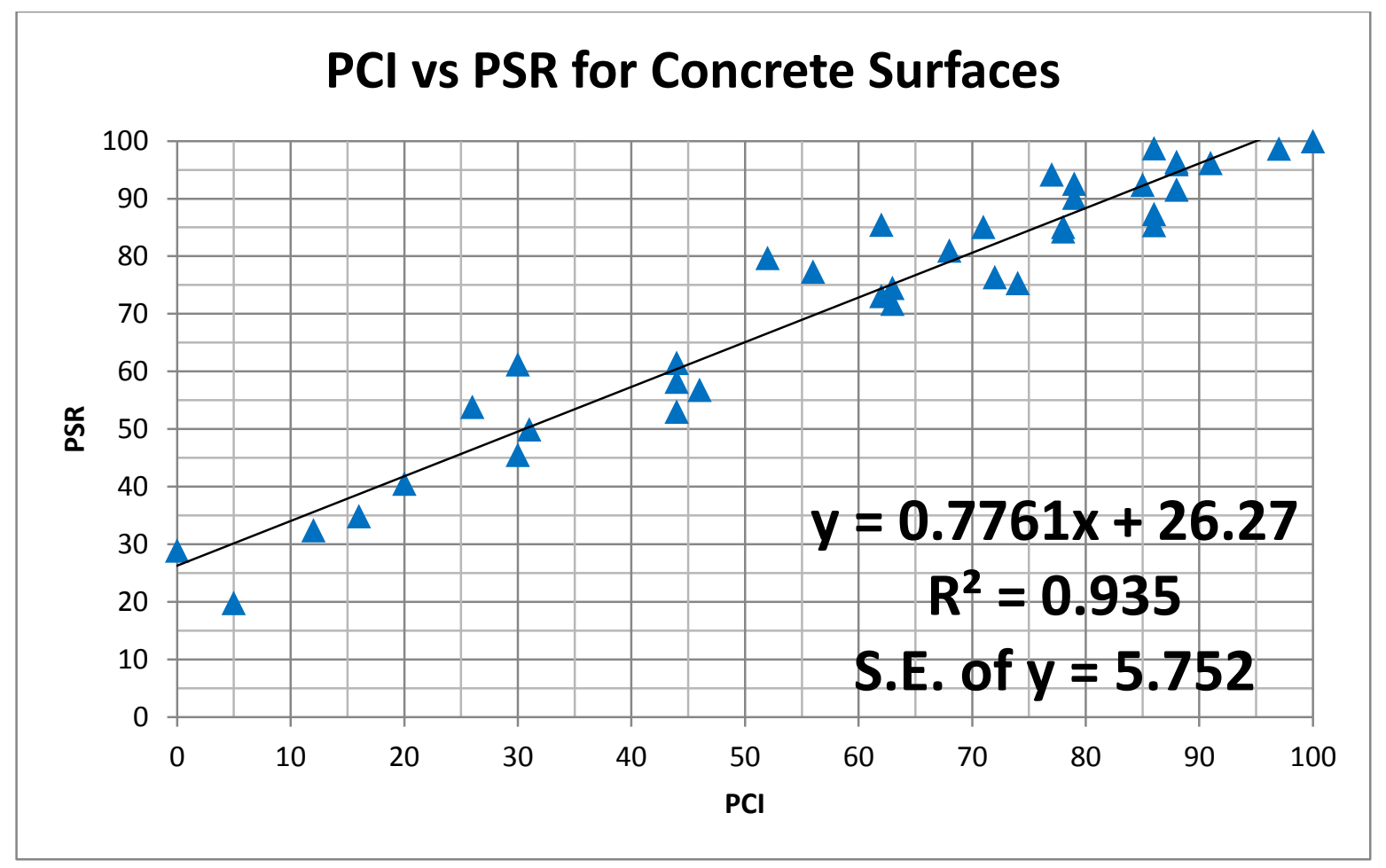

Figure 15: Final PCI vs. PSR Correlation for Concrete Surfaces

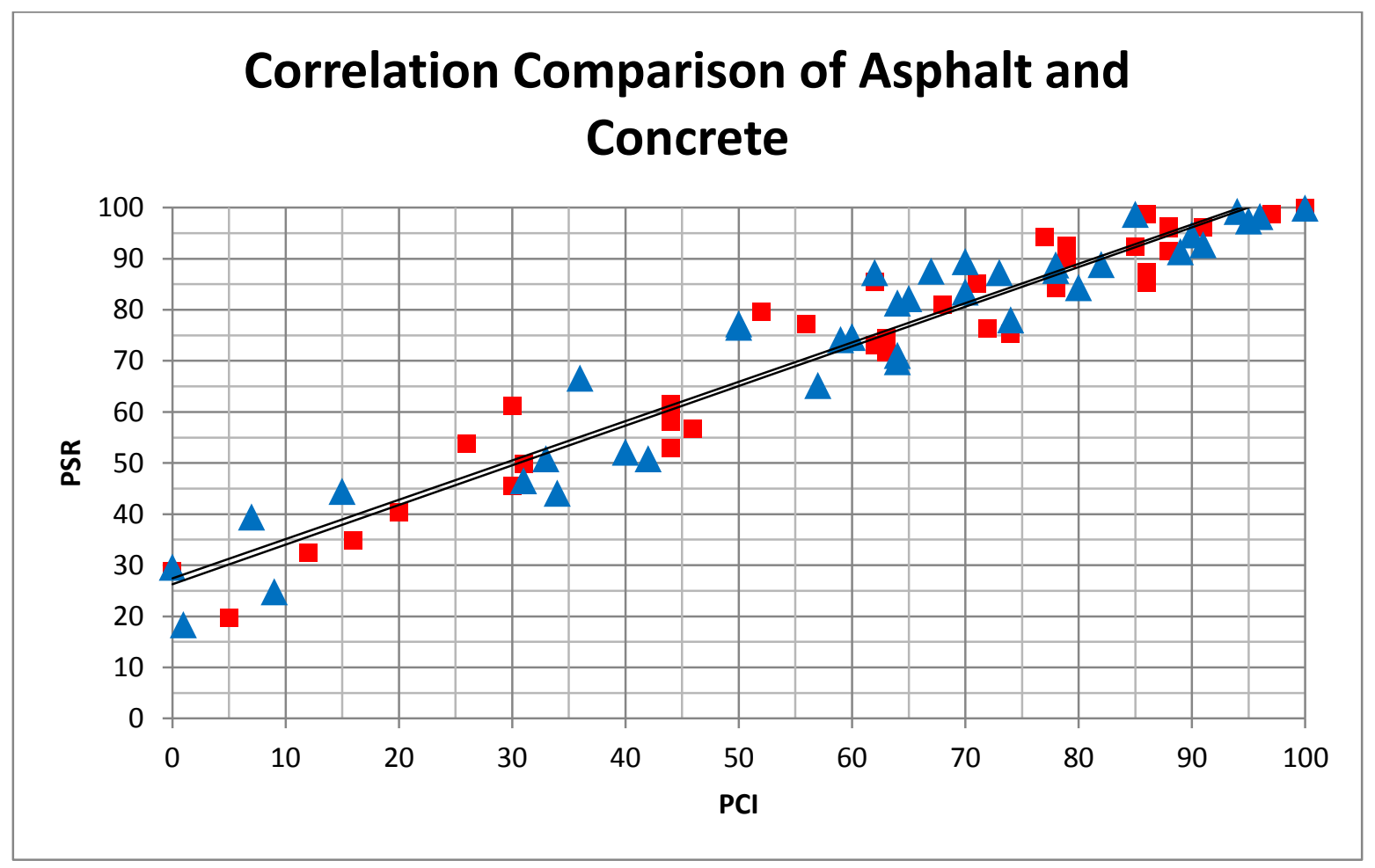

Figure 16: Combination of PCl vs. PSR Correlations to Compare Asphalt and Concrete 


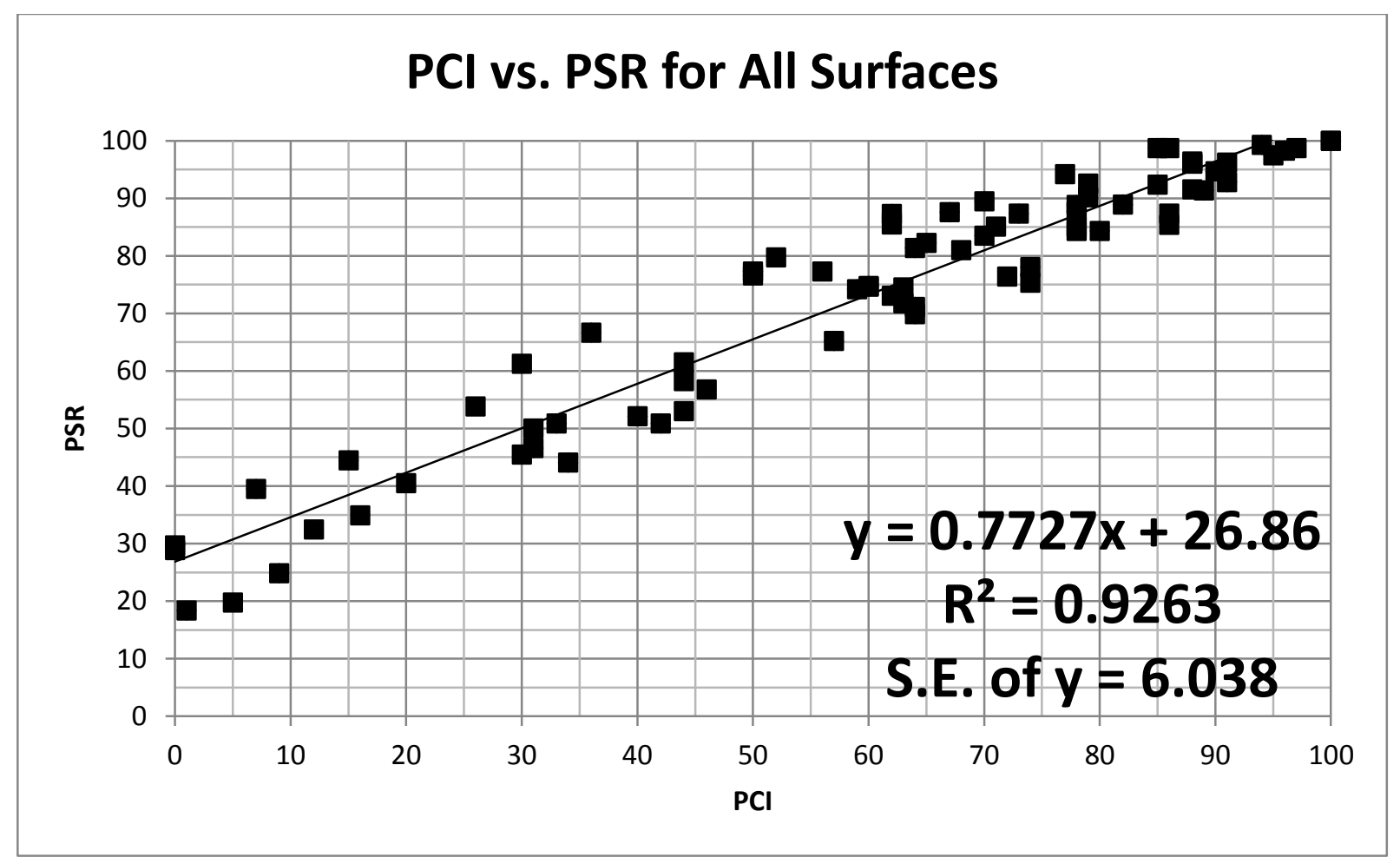

Figure 17: Combined PCI vs. PSR Correlations for all Pavement Surfaces

\subsubsection{Recommended PSR Procedure}

A distress identification manual was developed for determining PSR. Previously, Section 490, Special Provision included a few distress definitions and severity identifications, but generally deferred definition to the LTPP Distress Identification Manual. Inconsistencies in definitions and severities existed between the two documents. The new identification manual is exclusive to the calculation of the WVDOH PSR. It includes a distress definition, severity level identification scheme, and method for determining extent for every distress necessary to calculate PSR. The manual is titled "WVDOH Distress Identification Manual (for PSR calculation)" and is included as Appendix A.

Figure 18 is a blank asphalt surface test segment PSR calculation sheet. It contains an input for test section length, which should be 528 for any warranty jobs, but can be changed in the event that a segment is not $1 / 10$ mile so extents can be adjusted accordingly. Below that, a composite pavement identification input exists. A composite pavement is an asphalt overlay on an original concrete pavement. This has no effect on any calculation, but provides insight to anyone interpreting the distresses on diagrams or within the calculator. 
Figure 19 is a blank concrete surface test segment PSR calculation sheet. It too contains an input for test section length for the same reason as the asphalt PSR calculator. Additionally an input for number of transverse joints within the test segment is included. This is used in conjunction with the Normalized Weight Factor Column. Any distresses dependent on the presence of a joint are normalized to a 35 joint per $1 / 10$ miles basis. Previously, if a hypothetical concrete test section contained 80 foot slabs with six joints within a 1/10 mile section, one joint with low severity spalling would spike the extent calculation to $17 \%$. Although technically in this situation $17 \%$ of the joints in that test section were experiencing low severity spalling, this would affect the TWD and PSR the same way five low severity spalls would affect a 30 slab test section. In reality, five low spalls would be far worse than one spall. So now applicable distress weight factors are multiplied by a ratio of number of slabs divided by 35 , thus accounting for situations where there are non-uniform slab lengths, e.g. patched panels that create new joints within a section. This is actually an improvement on PCl's method which suggests only using test sections with 20 slabs to ensure the integrity of their deduct curves.

The weight factor is 0.0 for low severity transverse joint faults. This is because the definition for low severity faulting here is slightly more sensitive than that in the LTPP or PCI. The Special Provision warrant spec is to include a threshold for immediate corrective action for large amounts of faulting at any severity. For this reason, the low severity was left on the calculator spreadsheet to avoid changing the definition or removing a severity.

Compared to the 2012 PSR procedures, far more distresses on concrete segments are now measured by linear feet. This change accounts for non-uniform slab lengths. For most of these cases, the only difference is now instead of recording one slab affected by a distress, the length of the affected slab is recorded.

For both asphalt and concrete sections, the extent calculations are now based on the assumption that one transverse crack should affect a pavement the same amount that a distress at a joint of a concrete pavement would. Previously, extent was calculated by multiplying amount of transverse cracks by 2 to arrive at the extent percentage. This value is now 2.85 so that it too is normalized to a 35 joint per $1 / 10$ mile basis $(2.85 * 35=100)$. This means now a fault at a joint and a fault at a transverse crack in a concrete or asphalt surface pavement will have the same effect on PSR. 


\begin{tabular}{|c|c|c|c|c|c|c|c|}
\hline \multicolumn{8}{|c|}{ Asphalt Surface Segment PSR Calculator } \\
\hline & & & & \multirow{2}{*}{\multicolumn{3}{|c|}{$\begin{array}{c}\text { Test Section Length }(\mathrm{ft})= \\
\text { Composite Pavement? }\end{array}$}} & 528 \\
\hline & & & & & & & $\mathrm{Y} / \mathrm{N}$ \\
\hline & Distress & Severity & $\begin{array}{l}\text { Weight } \\
\text { Factor }\end{array}$ & $\begin{array}{c}\text { Amount } \\
\text { (\#) }\end{array}$ & $\begin{array}{l}\text { Length } \\
(\mathrm{ft})\end{array}$ & $\begin{array}{c}\text { Extent } \\
(\%)\end{array}$ & $\begin{array}{c}\text { Weighted } \\
\text { Distress }\end{array}$ \\
\hline \multirow{3}{*}{$\# 1$} & \multirow{3}{*}{ Transverse Cracks } & Low & 0.020 & & - & $0 \%$ & 0.00 \\
\hline & & Medium & 0.110 & & - & $0 \%$ & 0.00 \\
\hline & & High & 0.200 & & - & $0 \%$ & 0.00 \\
\hline \multirow{3}{*}{ \#2 } & \multirow{3}{*}{ Edge Cracking } & Low & 0.015 & - & & $0 \%$ & 0.00 \\
\hline & & Medium & 0.025 & - & & $0 \%$ & 0.00 \\
\hline & & High & 0.080 & - & & $0 \%$ & 0.00 \\
\hline \multirow{3}{*}{ \#3 } & \multirow{3}{*}{ Alligator Cracking } & Low & 0.140 & - & & $0 \%$ & 0.00 \\
\hline & & Medium & 0.240 & - & & $0 \%$ & 0.00 \\
\hline & & High & 0.340 & - & & $0 \%$ & 0.00 \\
\hline \multirow{3}{*}{ \#4 } & \multirow{3}{*}{ Block Cracking } & Low & 0.055 & - & & $0 \%$ & 0.00 \\
\hline & & Medium & 0.105 & - & & $0 \%$ & 0.00 \\
\hline & & High & 0.165 & - & & $0 \%$ & 0.00 \\
\hline \multirow{3}{*}{ \#5 } & \multirow{3}{*}{ Longitudinal Cracking } & Low & 0.020 & - & & $0 \%$ & 0.00 \\
\hline & & Medium & 0.030 & - & & $0 \%$ & 0.00 \\
\hline & & High & 0.060 & - & & $0 \%$ & 0.00 \\
\hline \multirow{3}{*}{ \#6 } & \multirow{3}{*}{$\begin{array}{l}\text { Longitudinal Joint } \\
\text { Deterioration }\end{array}$} & Low & 0.025 & - & & $0 \%$ & 0.00 \\
\hline & & Medium & 0.045 & - & & $0 \%$ & 0.00 \\
\hline & & High & 0.080 & - & & $0 \%$ & 0.00 \\
\hline \multirow{3}{*}{$\# 7$} & \multirow{3}{*}{$\begin{array}{c}\text { Lane Edge Cracking/ } \\
\text { Deterioration }\end{array}$} & Low & 0.010 & - & & $0 \%$ & 0.00 \\
\hline & & Medium & 0.020 & - & & $0 \%$ & 0.00 \\
\hline & & High & 0.040 & - & & $0 \%$ & 0.00 \\
\hline \#8 & Raveling/Weathering & - & 0.040 & - & & $0 \%$ & 0.00 \\
\hline$\# 9$ & Patching & - & 0.030 & - & & $0 \%$ & 0.00 \\
\hline \multirow{2}{*}{$\# 10$} & \multirow{2}{*}{ Rutting } & Low & 0.065 & - & & $0 \%$ & 0.00 \\
\hline & & High & 0.150 & - & & $0 \%$ & 0.00 \\
\hline & & & & \multicolumn{3}{|c|}{ Segment TWD = } & 0.00 \\
\hline & & & & \multicolumn{3}{|c|}{ Segment PSR = } & 100.0 \\
\hline
\end{tabular}

Figure 18: Blank Asphalt Surface Segment PSR Calculator 


\begin{tabular}{|c|c|c|c|c|c|c|c|c|}
\hline \multicolumn{9}{|c|}{ Concrete Surface Segment PSR Calculator } \\
\hline & & & & & \multicolumn{3}{|c|}{$\begin{array}{c}\text { Test Section Length }(\mathrm{ft})= \\
\text { Joints in Segment }(\#)=\end{array}$} & $\begin{array}{c}528 \\
35\end{array}$ \\
\hline & Distress & Severity & $\begin{array}{l}\text { Weight } \\
\text { Factor }\end{array}$ & $\begin{array}{l}\text { Amount/ } \\
\text { Joints (\#) }\end{array}$ & $\begin{array}{l}\text { Length } \\
(\mathrm{ft})\end{array}$ & $\begin{array}{c}\text { Extent } \\
(\%)\end{array}$ & $\begin{array}{c}\text { Normalized } \\
\text { WF }\end{array}$ & $\begin{array}{c}\text { Weighted } \\
\text { Distress }\end{array}$ \\
\hline \multirow{3}{*}{ \#11 } & \multirow{3}{*}{ Transverse Cracks } & Low & 0.040 & & - & $0 \%$ & 0.040 & 0.00 \\
\hline & & Medium & 0.100 & & - & $0 \%$ & 0.100 & 0.00 \\
\hline & & High & 0.210 & & - & $0 \%$ & 0.210 & 0.00 \\
\hline \multirow{3}{*}{ \#12 } & \multirow{3}{*}{$\begin{array}{c}\text { Transverse Joints } \\
\text { Spalls }\end{array}$} & Low & 0.020 & & - & $0 \%$ & 0.020 & 0.00 \\
\hline & & Medium & 0.075 & & - & $0 \%$ & 0.075 & 0.00 \\
\hline & & High & 0.150 & & - & $0 \%$ & 0.150 & 0.00 \\
\hline \multirow{3}{*}{ \#13 } & \multirow{3}{*}{$\begin{array}{l}\text { Transverse Joint } \\
\text { Faults }\end{array}$} & Low & 0.000 & & - & $0 \%$ & 0.000 & 0.00 \\
\hline & & Medium & 0.110 & & - & $0 \%$ & 0.110 & 0.00 \\
\hline & & High & 0.210 & & - & $0 \%$ & 0.210 & 0.00 \\
\hline \#14 & Blowups & - & 0.250 & & - & $0 \%$ & 0.250 & 0.00 \\
\hline \multirow{3}{*}{ \#15 } & \multirow{3}{*}{$\begin{array}{l}\text { Longitudinal } \\
\text { Cracking }\end{array}$} & Low & 0.030 & - & & $0 \%$ & 0.030 & 0.00 \\
\hline & & Medium & 0.090 & - & & $0 \%$ & 0.090 & 0.00 \\
\hline & & High & 0.190 & - & & $0 \%$ & 0.190 & 0.00 \\
\hline \multirow{3}{*}{ \#16 } & \multirow{3}{*}{$\begin{array}{l}\text { Longitudinal Joint } \\
\text { Spalling }\end{array}$} & Low & 0.020 & - & & $0 \%$ & 0.020 & 0.00 \\
\hline & & Medium & 0.060 & - & & $0 \%$ & 0.060 & 0.00 \\
\hline & & High & 0.150 & - & & $0 \%$ & 0.150 & 0.00 \\
\hline \multirow{3}{*}{ \#17 } & \multirow{3}{*}{ Lane Edge Spalling } & Low & 0.010 & - & & $0 \%$ & 0.010 & 0.00 \\
\hline & & Medium & 0.050 & - & & $0 \%$ & 0.050 & 0.00 \\
\hline & & High & 0.150 & - & & $0 \%$ & 0.150 & 0.00 \\
\hline$\# 18$ & D Cracking & - & 0.100 & - & & $0 \%$ & 0.100 & 0.00 \\
\hline \#19 & Scaling & - & 0.020 & - & & $0 \%$ & 0.020 & 0.00 \\
\hline$\# 20$ & Patching & - & 0.030 & - & & $0 \%$ & 0.030 & 0.00 \\
\hline$\# 21$ & Popouts & - & 0.035 & - & & $0 \%$ & 0.035 & 0.00 \\
\hline & & & & & \multicolumn{3}{|c|}{ Segment TWD = } & 0.00 \\
\hline & & & & & \multicolumn{3}{|c|}{ Segment PSR = } & 100.0 \\
\hline
\end{tabular}

Figure 19: Blank Concrete Surface Segment PSR Calculator

\subsubsection{US 35 PSR with Recommended Method}

Table 30 and Table 31 contain a comprehensive summary of all final calculated PSR values for each of the five surveys at every test section observed. Figure 20 through Figure 35 graphically describe the data within these tables by analyzing the five surveys by lane, direction, and pavement type. For each combination of those three variables there is a figure which analyzes Pavement Age vs. PSR and a 
figure which analyzes Mile Marker vs. PSR. Pavement Age vs. PSR plots the observed PSR values for all applicable test sections over the life of the pavement in months. Mile Marker vs. PSR plots observed PSR for each of the five surveys and is grouped by test section mile markers.

\begin{tabular}{|c|c|c|c|c|c|c|c|}
\hline & & Age (Months) & 19 & 24 & 30 & 35 & 43 \\
\hline & & Mile Marker & Survey 1 & Survey 2 & Survey 3 & Survey 4 & Survey 5 \\
\hline \multirow{14}{*}{$\begin{array}{c}\text { Concrete } \\
\text { NB }\end{array}$} & \multirow{7}{*}{$\begin{array}{l}\text { Slow } \\
\text { Lane }\end{array}$} & $9.1-9.2 \mathrm{SL}$ & 97.94 & 95.74 & 95.74 & 95.47 & 95.23 \\
\hline & & $9.4-9.5 \mathrm{SL}$ & 99.97 & 97.68 & 97.68 & 97.18 & 96.44 \\
\hline & & 10.4 - $10.5 \mathrm{SL}$ & 99.34 & 98.48 & 97.95 & 97.04 & 94.22 \\
\hline & & $10.8-10.9 \mathrm{SL}$ & 99.96 & 98.14 & 97.13 & 96.88 & 95.65 \\
\hline & & $11.4-11.5 \mathrm{SL}$ & 99.97 & 99.71 & 99.46 & 97.37 & 97.37 \\
\hline & & $11.7-11.8 \mathrm{SL}$ & 99.97 & 99.94 & 99.94 & 99.67 & 99.67 \\
\hline & & $12.2-12.3 \mathrm{SL}$ & 99.33 & 99.33 & 99.32 & 99.01 & 98.73 \\
\hline & \multirow{7}{*}{$\begin{array}{l}\text { Fast } \\
\text { Lane }\end{array}$} & $9.1-9.2 \mathrm{FL}$ & 99.97 & 99.97 & 99.97 & 99.46 & 99.46 \\
\hline & & $9.4-9.5 \mathrm{FL}$ & 99.97 & 97.18 & 97.18 & 97.18 & 96.68 \\
\hline & & 10.4 - $10.5 \mathrm{FL}$ & 99.71 & 98.95 & 95.94 & 95.20 & 93.25 \\
\hline & & 10.8 - 10.9 FL & 99.97 & 95.20 & 95.20 & 94.72 & 94.42 \\
\hline & & $11.4-11.5 \mathrm{FL}$ & 99.70 & 99.19 & 98.68 & 96.92 & 96.92 \\
\hline & & $11.7-11.8 \mathrm{FL}$ & 99.71 & 98.95 & 98.44 & 97.18 & 97.18 \\
\hline & & $12.2-12.3 \mathrm{FL}$ & 99.01 & 99.00 & 98.49 & 97.24 & 97.24 \\
\hline \multirow{23}{*}{$\begin{array}{c}\text { Concrete } \\
\text { SB }\end{array}$} & \multirow{7}{*}{$\begin{array}{l}\text { Slow } \\
\text { Lane }\end{array}$} & $9.3-9.2 \mathrm{SL}$ & 94.23 & 94.23 & 93.75 & 93.75 & 93.27 \\
\hline & & $9.5-9.4 \mathrm{SL}$ & 96.18 & 94.94 & 94.46 & 91.82 & 91.59 \\
\hline & & 10.5 - 10.4 SL & 99.71 & 99.71 & 99.71 & 97.68 & 97.68 \\
\hline & & $10.9-10.8 \mathrm{SL}$ & 99.44 & 99.44 & 98.93 & 95.92 & 95.92 \\
\hline & & $11.6-11.5 \mathrm{SL}$ & 99.87 & 99.87 & 99.87 & 97.82 & 97.82 \\
\hline & & $11.8-11.7 \mathrm{SL}$ & 99.61 & 99.37 & 99.37 & 99.33 & 99.33 \\
\hline & & $12.35-12.25 \mathrm{SL}$ & 99.66 & 99.65 & 99.65 & 99.65 & 99.65 \\
\hline & \multirow{7}{*}{$\begin{array}{l}\text { Fast } \\
\text { Lane }\end{array}$} & $9.3-9.2 \mathrm{FL}$ & 99.20 & 98.19 & 96.19 & 94.96 & 94.47 \\
\hline & & $9.5-9.4 \mathrm{FL}$ & 97.17 & 97.17 & 97.17 & 95.19 & 93.95 \\
\hline & & 10.5 - 10.4 FL & 99.97 & 99.97 & 99.97 & 97.43 & 96.68 \\
\hline & & $10.9-10.8 \mathrm{FL}$ & 99.71 & 99.46 & 98.69 & 97.94 & 97.94 \\
\hline & & 11.6 - 11.5 FL & 99.97 & 99.97 & 99.97 & 99.97 & 99.97 \\
\hline & & 11.8 - 11.7 FL & 99.65 & 99.39 & 99.39 & 99.39 & 99.39 \\
\hline & & $12.35-12.25 \mathrm{FL}$ & 99.97 & 99.97 & 99.97 & 99.71 & 99.71 \\
\hline & & PCC NB SL Avg & 99.50 & 98.43 & 98.17 & 97.52 & 96.76 \\
\hline & & PCC NB FL Avg & 99.72 & 98.35 & 97.70 & 96.84 & 96.45 \\
\hline & & PCC SB SL Avg & 98.39 & 98.17 & 97.96 & 96.57 & 96.47 \\
\hline & & PCC SB FL Avg & 99.38 & 99.16 & 98.76 & 97.80 & 97.44 \\
\hline & & PCC NB Avg & 99.61 & 98.39 & 97.94 & 97.18 & 96.60 \\
\hline & & PCC SB Avg & 98.88 & 98.67 & 98.36 & 97.18 & 96.96 \\
\hline & & PCC SL Avg & 98.94 & 98.30 & 98.07 & 97.04 & 96.61 \\
\hline & & PCC FL Avg & 99.55 & 98.75 & 98.23 & 97.32 & 96.95 \\
\hline & & PCC Avg & 99.25 & 98.53 & 98.15 & 97.18 & 96.78 \\
\hline
\end{tabular}

Table 30: Summary of US 35 Concrete Section PSR 


\begin{tabular}{|c|c|c|c|c|c|c|c|}
\hline & & Age (Months) & 19 & 24 & 30 & 35 & 43 \\
\hline & & Mile Marker & Survey 1 & Survey 2 & Survey 3 & Survey 4 & Survey 5 \\
\hline \multirow{18}{*}{$\begin{array}{c}\text { Asphalt } \\
\text { NB }\end{array}$} & \multirow{9}{*}{$\begin{array}{l}\text { Slow } \\
\text { Lane }\end{array}$} & $12.6-12.7 \mathrm{SL}$ & 97.70 & 97.70 & 89.33 & 89.31 & 85.38 \\
\hline & & $13.2-13.3 \mathrm{SL}$ & 97.86 & 98.05 & 93.46 & 91.41 & 91.41 \\
\hline & & $13.8-13.9 \mathrm{SL}$ & 99.80 & 99.73 & 98.80 & 97.88 & 89.33 \\
\hline & & $14.4-14.5 \mathrm{SL}$ & 98.81 & 98.81 & 96.00 & 96.00 & 95.25 \\
\hline & & $14.6-14.7 \mathrm{SL}$ & 99.97 & 99.97 & 99.97 & 98.36 & 92.23 \\
\hline & & $15.2-15.3 \mathrm{SL}$ & 98.51 & 98.30 & 91.53 & 86.24 & 86.24 \\
\hline & & $15.4-15.5 \mathrm{SL}$ & 98.93 & 98.35 & 95.18 & 90.92 & 74.75 \\
\hline & & $16.0-16.1 \mathrm{SL}$ & 98.89 & 97.86 & 95.86 & 95.50 & 83.66 \\
\hline & & $16.4-16.5 \mathrm{SL}$ & 98.70 & 98.70 & 97.90 & 96.62 & 84.42 \\
\hline & \multirow{9}{*}{$\begin{array}{l}\text { Fast } \\
\text { Lane }\end{array}$} & $12.6-12.7 \mathrm{FL}$ & 99.97 & 99.97 & 99.97 & 99.97 & 91.37 \\
\hline & & $13.2-13.3 \mathrm{FL}$ & 99.97 & 99.97 & 99.97 & 99.97 & 92.33 \\
\hline & & $13.8-13.9 \mathrm{FL}$ & 99.97 & 99.97 & 99.97 & 99.97 & 96.80 \\
\hline & & $14.4-14.5 \mathrm{FL}$ & 99.97 & 99.97 & 99.97 & 99.97 & 99.97 \\
\hline & & $14.6-14.7 \mathrm{FL}$ & 99.97 & 99.97 & 99.97 & 99.97 & 99.97 \\
\hline & & $15.2-15.3 \mathrm{FL}$ & 99.97 & 99.97 & 99.97 & 99.97 & 88.58 \\
\hline & & $15.4-15.5 \mathrm{FL}$ & 99.97 & 99.97 & 99.97 & 99.97 & 89.38 \\
\hline & & $16.0-16.1 \mathrm{FL}$ & 99.97 & 99.97 & 99.97 & 99.97 & 88.82 \\
\hline & & $16.4-16.5 \mathrm{FL}$ & 99.97 & 99.97 & 99.97 & 99.97 & 99.97 \\
\hline \multirow{27}{*}{$\begin{array}{l}\text { Asphalt } \\
\text { SB }\end{array}$} & \multirow{9}{*}{$\begin{array}{l}\text { Slow } \\
\text { Lane }\end{array}$} & $12.7-12.6 \mathrm{SL}$ & 98.00 & 98.00 & 98.00 & 93.59 & 88.42 \\
\hline & & $13.3-13.2 \mathrm{SL}$ & 98.03 & 97.74 & 95.08 & 93.20 & 81.38 \\
\hline & & $13.9-13.8 \mathrm{SL}$ & 99.29 & 99.29 & 98.34 & 92.37 & 90.19 \\
\hline & & $14.5-14.4 \mathrm{SL}$ & 99.61 & 99.61 & 99.13 & 92.78 & 89.51 \\
\hline & & $14.7-14.6 \mathrm{SL}$ & 99.97 & 99.97 & 98.08 & 94.91 & 94.91 \\
\hline & & $15.3-15.2 \mathrm{SL}$ & 98.42 & 97.48 & 93.98 & 88.70 & 85.58 \\
\hline & & $15.5-15.4 \mathrm{SL}$ & 98.63 & 98.63 & 98.63 & 96.36 & 85.80 \\
\hline & & $16.1-16.0 \mathrm{SL}$ & 99.33 & 98.47 & 97.05 & 97.05 & 97.05 \\
\hline & & $16.5-16.4 \mathrm{SL}$ & 99.97 & 99.27 & 93.68 & 93.68 & 89.47 \\
\hline & \multirow{9}{*}{$\begin{array}{l}\text { Fast } \\
\text { Lane }\end{array}$} & $12.7-12.6 \mathrm{FL}$ & 99.97 & 97.60 & 97.60 & 97.60 & 97.60 \\
\hline & & 13.3-13.2 FL & 99.97 & 99.97 & 99.97 & 99.97 & 87.35 \\
\hline & & $13.9-13.8 \mathrm{FL}$ & 95.57 & 95.57 & 95.57 & 95.57 & 83.50 \\
\hline & & $14.5-14.4 \mathrm{FL}$ & 99.97 & 99.97 & 99.97 & 99.55 & 87.06 \\
\hline & & $14.7-14.6 \mathrm{FL}$ & 99.97 & 99.97 & 99.97 & 99.97 & 99.97 \\
\hline & & $15.3-15.2 \mathrm{FL}$ & 98.74 & 98.74 & 98.74 & 98.74 & 90.18 \\
\hline & & $15.5-15.4 \mathrm{FL}$ & 99.97 & 99.97 & 99.97 & 99.97 & 95.33 \\
\hline & & $16.1-16.0 \mathrm{FL}$ & 99.97 & 99.97 & 99.97 & 99.97 & 99.97 \\
\hline & & $16.5-16.4 \mathrm{FL}$ & 99.97 & 99.97 & 99.97 & 99.97 & 99.97 \\
\hline & & HMA NB SL Avg & 98.80 & 98.61 & 95.34 & 93.58 & 86.96 \\
\hline & & HMA NB FL Avg & 99.97 & 99.97 & 99.97 & 99.97 & 94.13 \\
\hline & & HMA SB SL Avg & 99.03 & 98.72 & 96.89 & 93.63 & 89.15 \\
\hline & & HMA SB FL Avg & 99.35 & 99.08 & 99.08 & 99.03 & 93.44 \\
\hline & & HMA NB Avg & 99.38 & 99.29 & 97.65 & 96.78 & 90.55 \\
\hline & & HMA SB Avg & 99.19 & 98.90 & 97.98 & 96.33 & 91.29 \\
\hline & & HMA SL Avg & 98.91 & 98.66 & 96.11 & 93.61 & 88.06 \\
\hline & & HMA FL Avg & 99.66 & 99.53 & 99.53 & 99.50 & 93.78 \\
\hline & & HMA Avg & 99.29 & 99.09 & 97.82 & 96.55 & 90.92 \\
\hline
\end{tabular}

Table 31: Summary of US 35 Asphalt Section PSR 


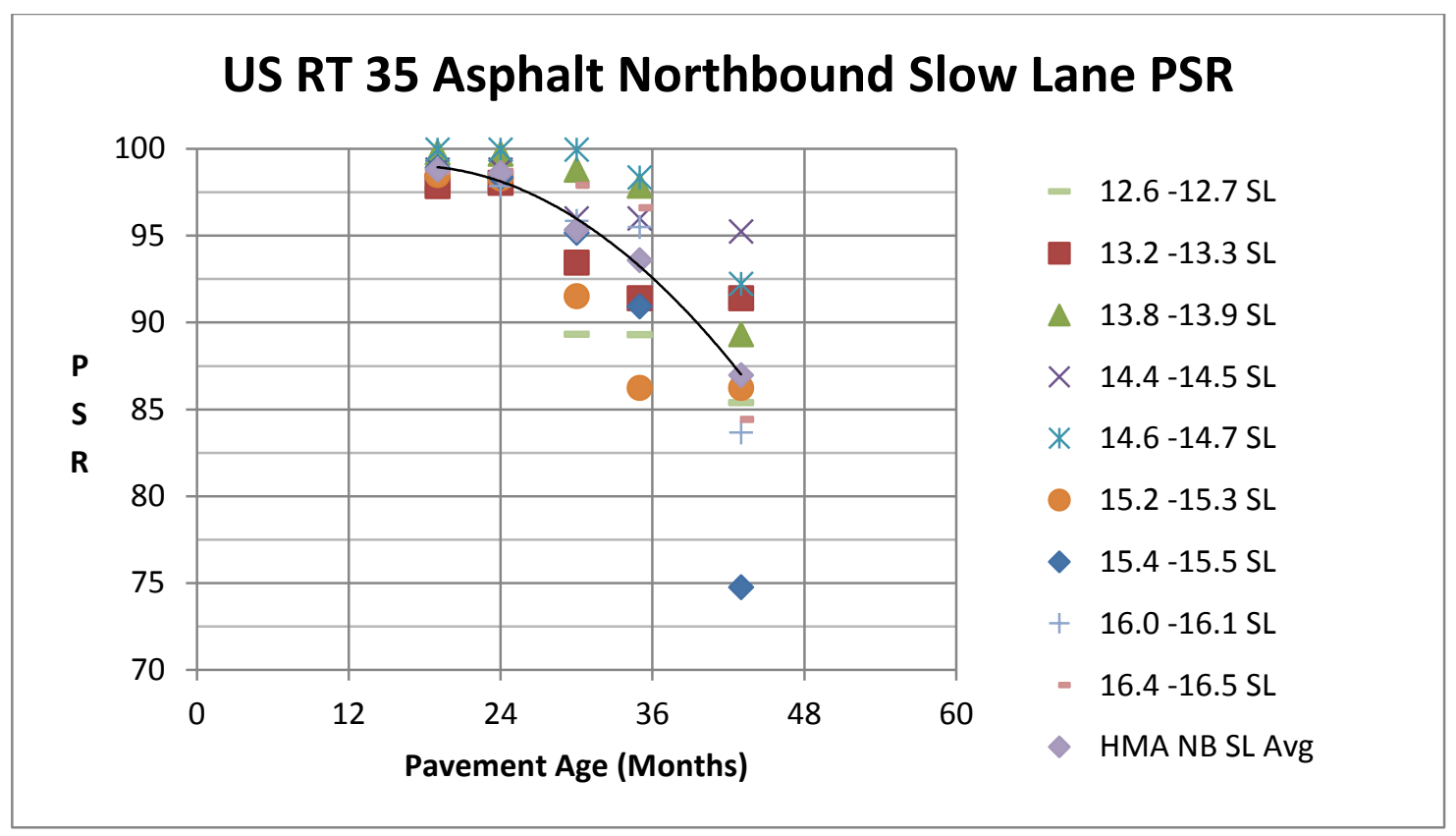

Figure 20: US 35 Asphalt Northbound Slow Lane Pavement Age vs. PSR

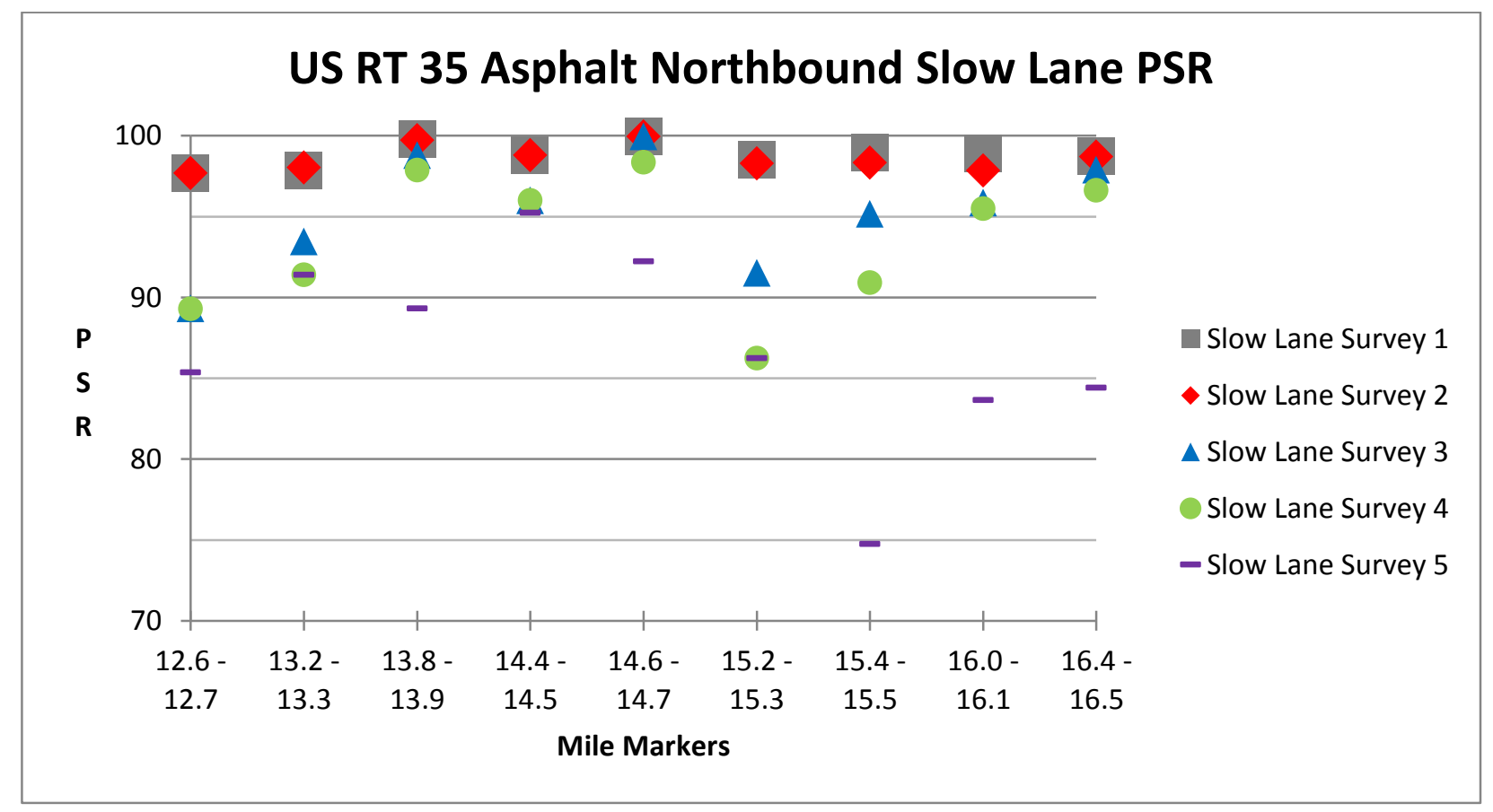

Figure 21: US 35 Asphalt Northbound Slow Lane Mile Marker vs. PSR 


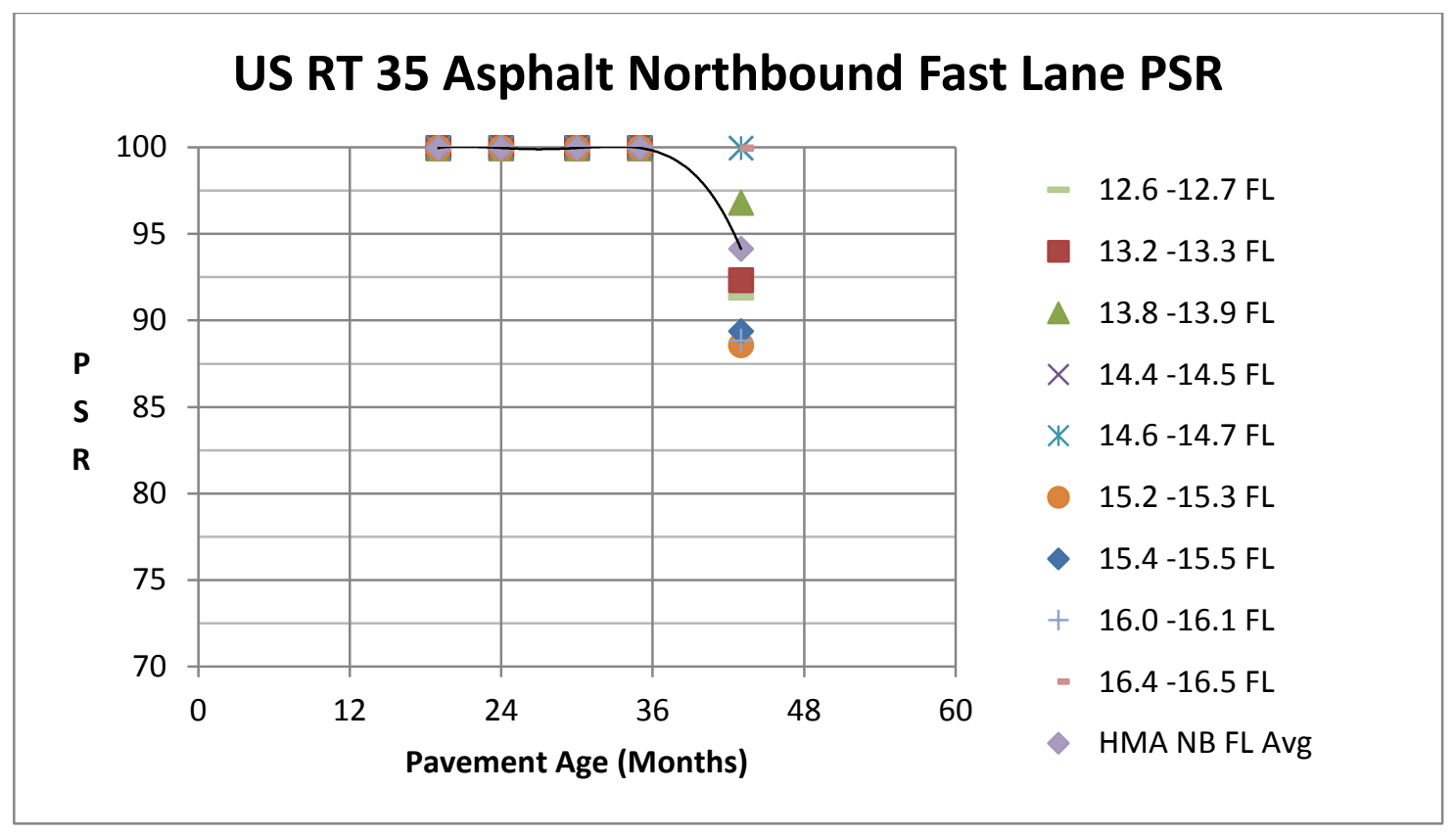

Figure 22: US 35 Asphalt Northbound Fast Lane Pavement Age vs. PSR

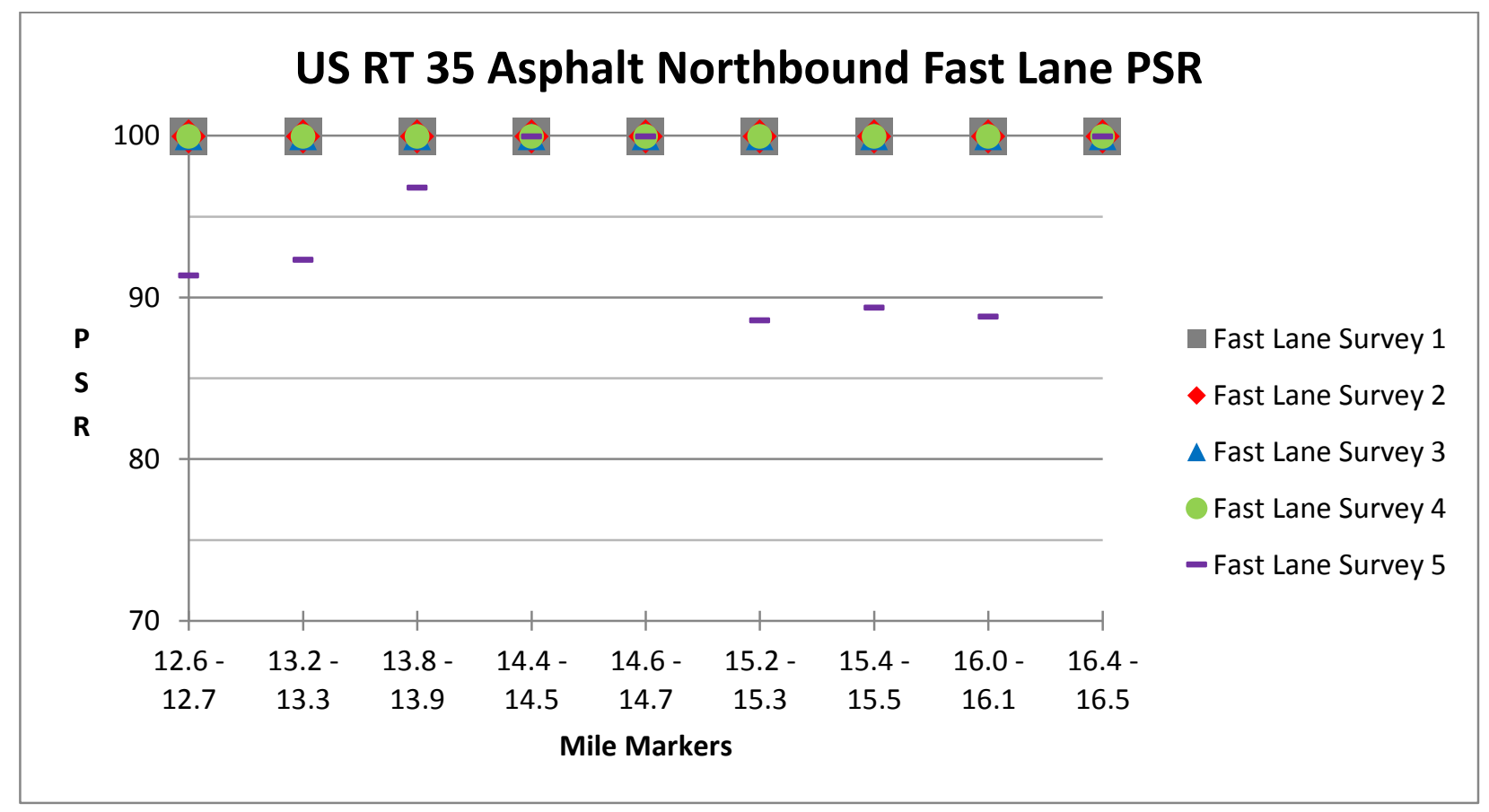

Figure 23: US 35 Asphalt Northbound Fast Lane Mile Marker vs. PSR 


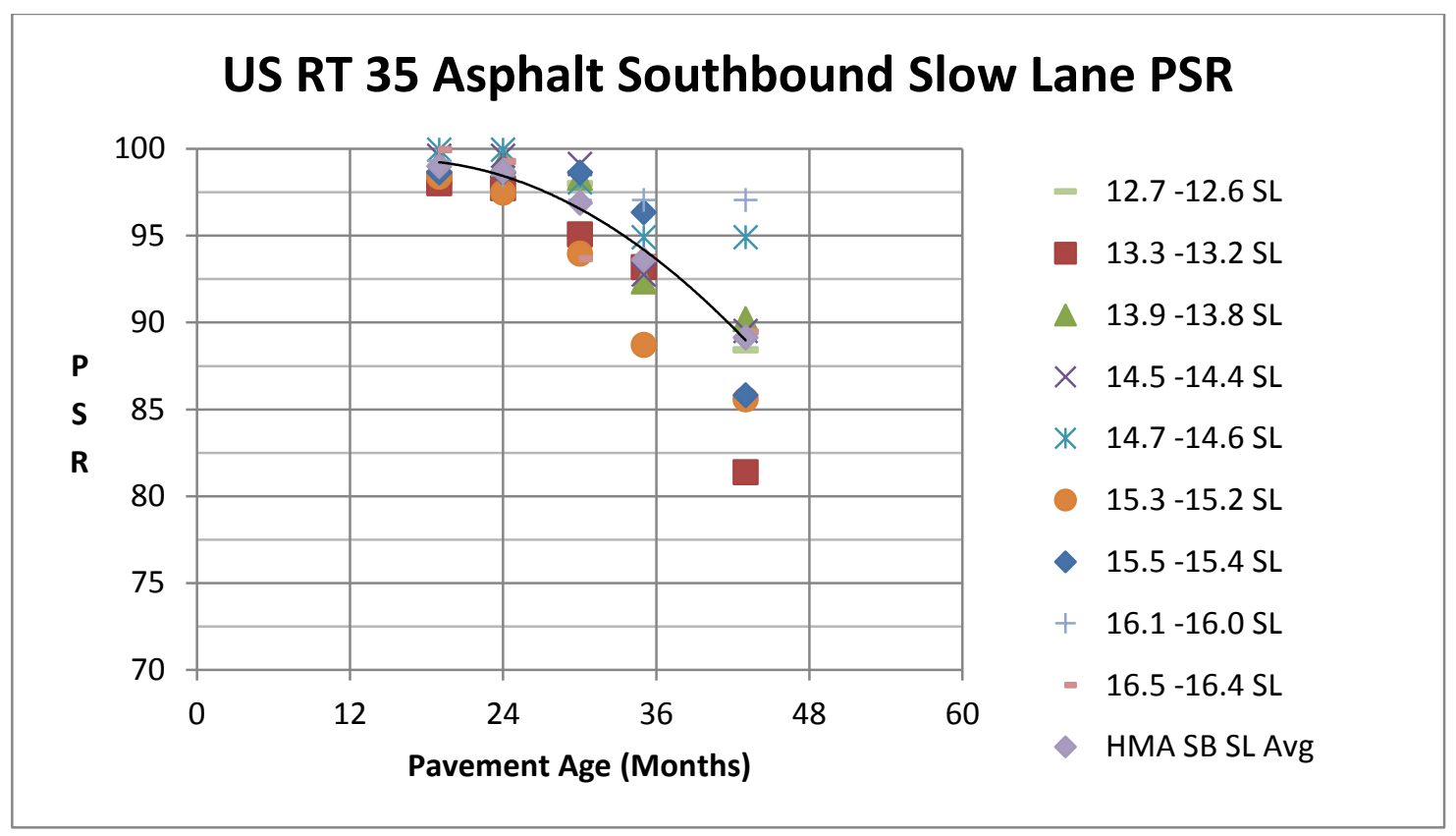

Figure 24: US 35 Asphalt Southbound Slow Lane Pavement Age vs. PSR

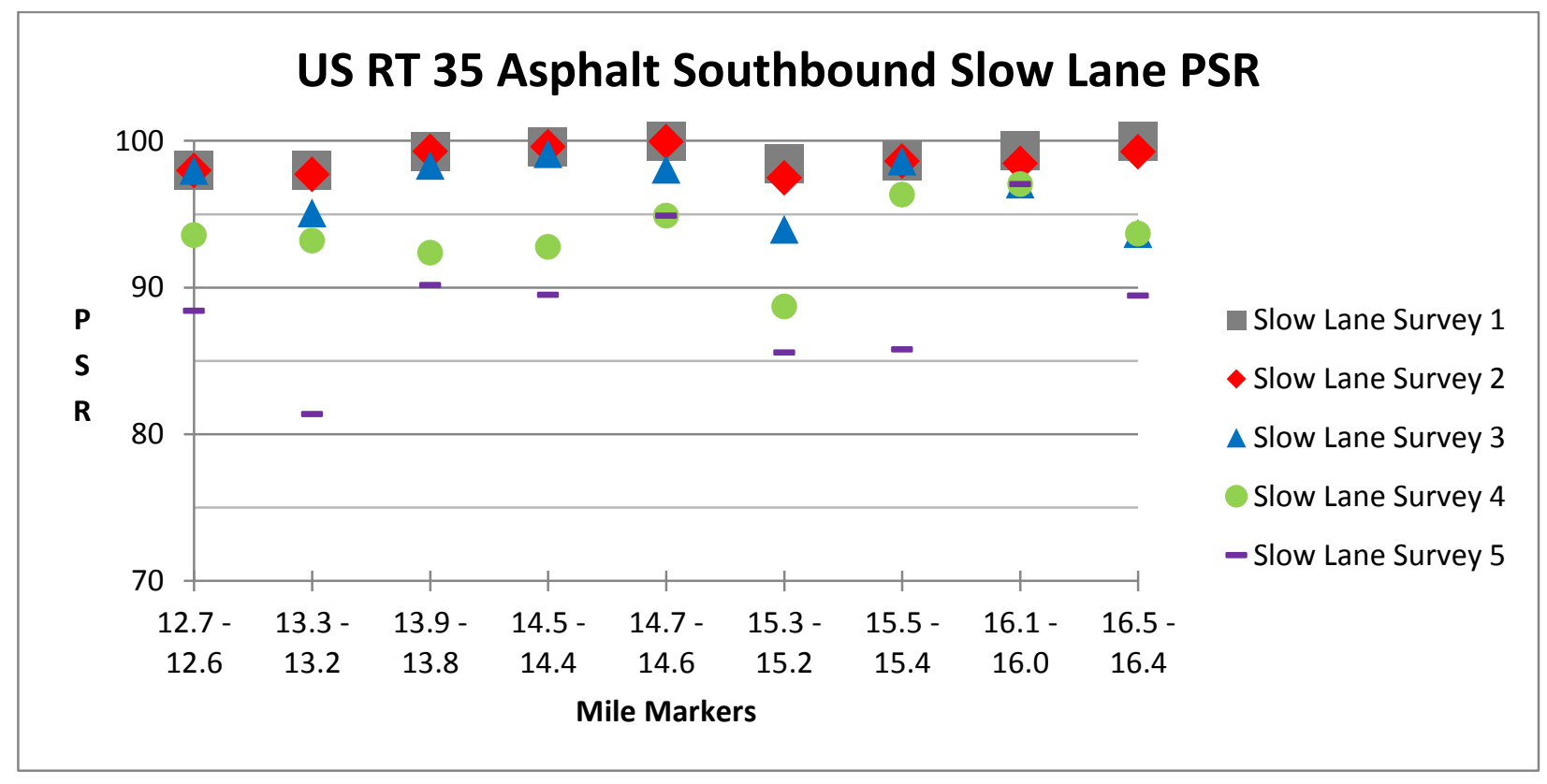

Figure 25: US 35 Asphalt Southbound Slow Lane Mile Marker vs. PSR 


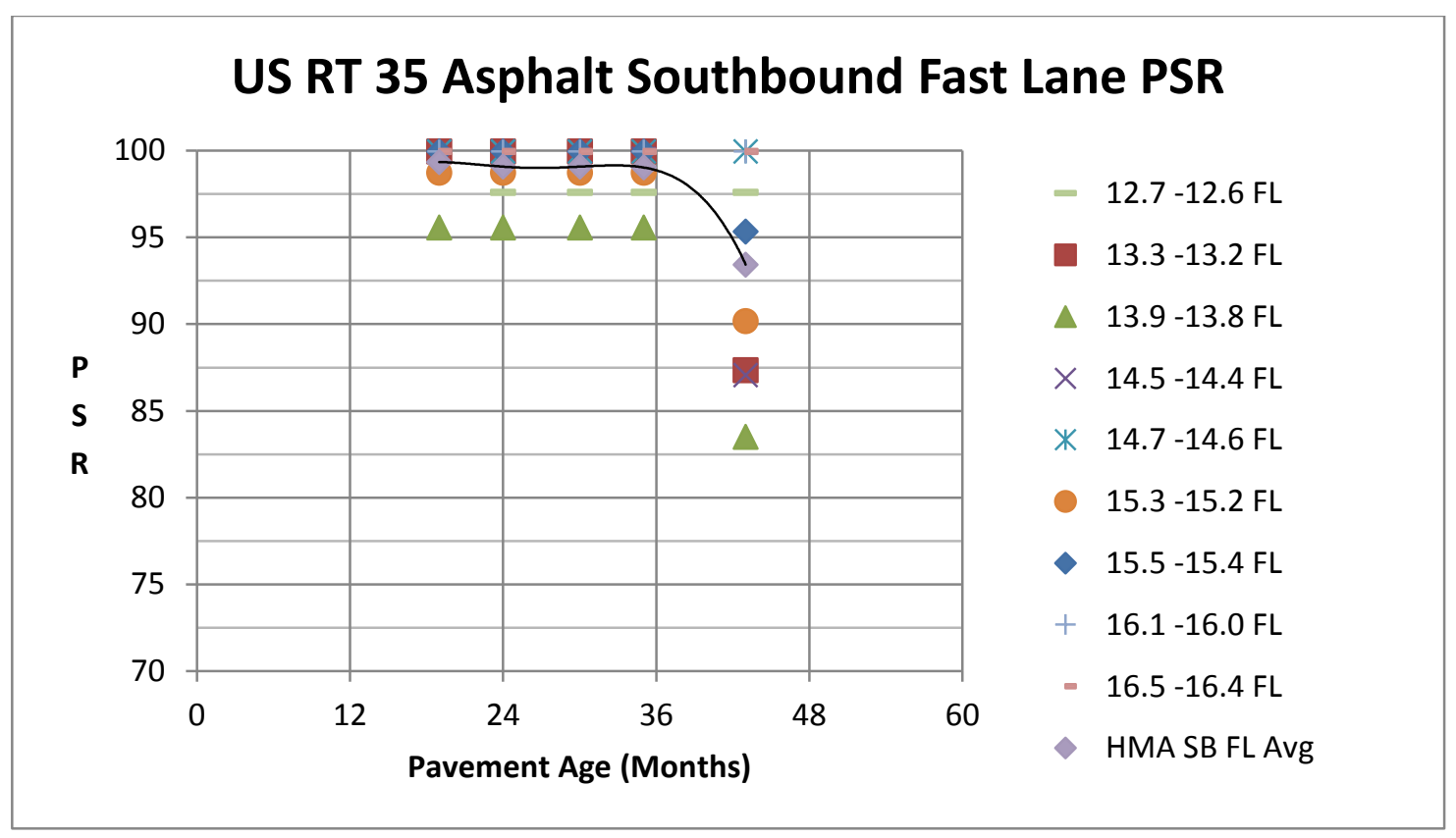

Figure 26: US 35 Asphalt Southbound Fast Lane Pavement Age vs. PSR

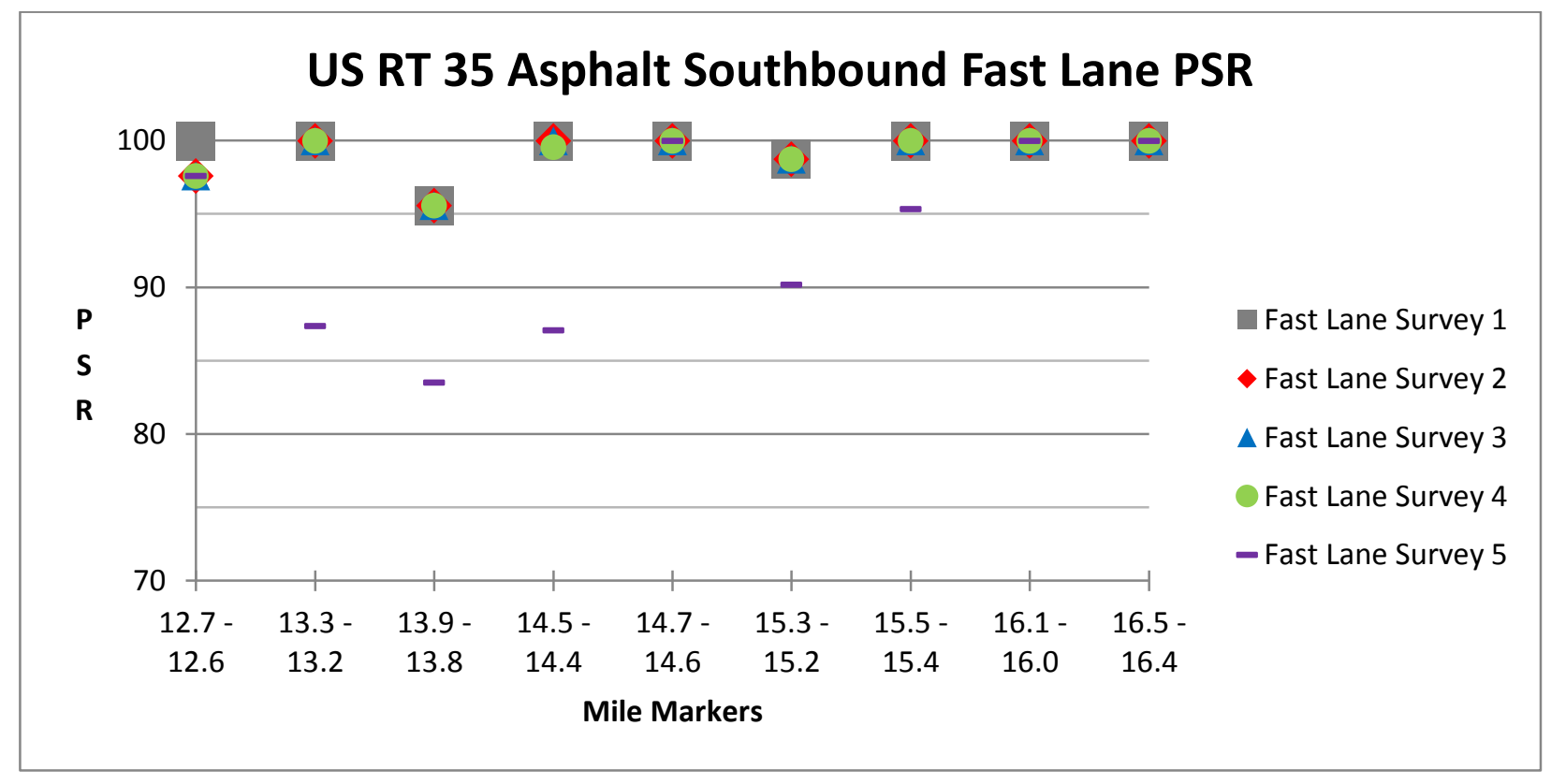

Figure 27: US 35 Asphalt Southbound Fast Lane Mile Marker vs. PSR 


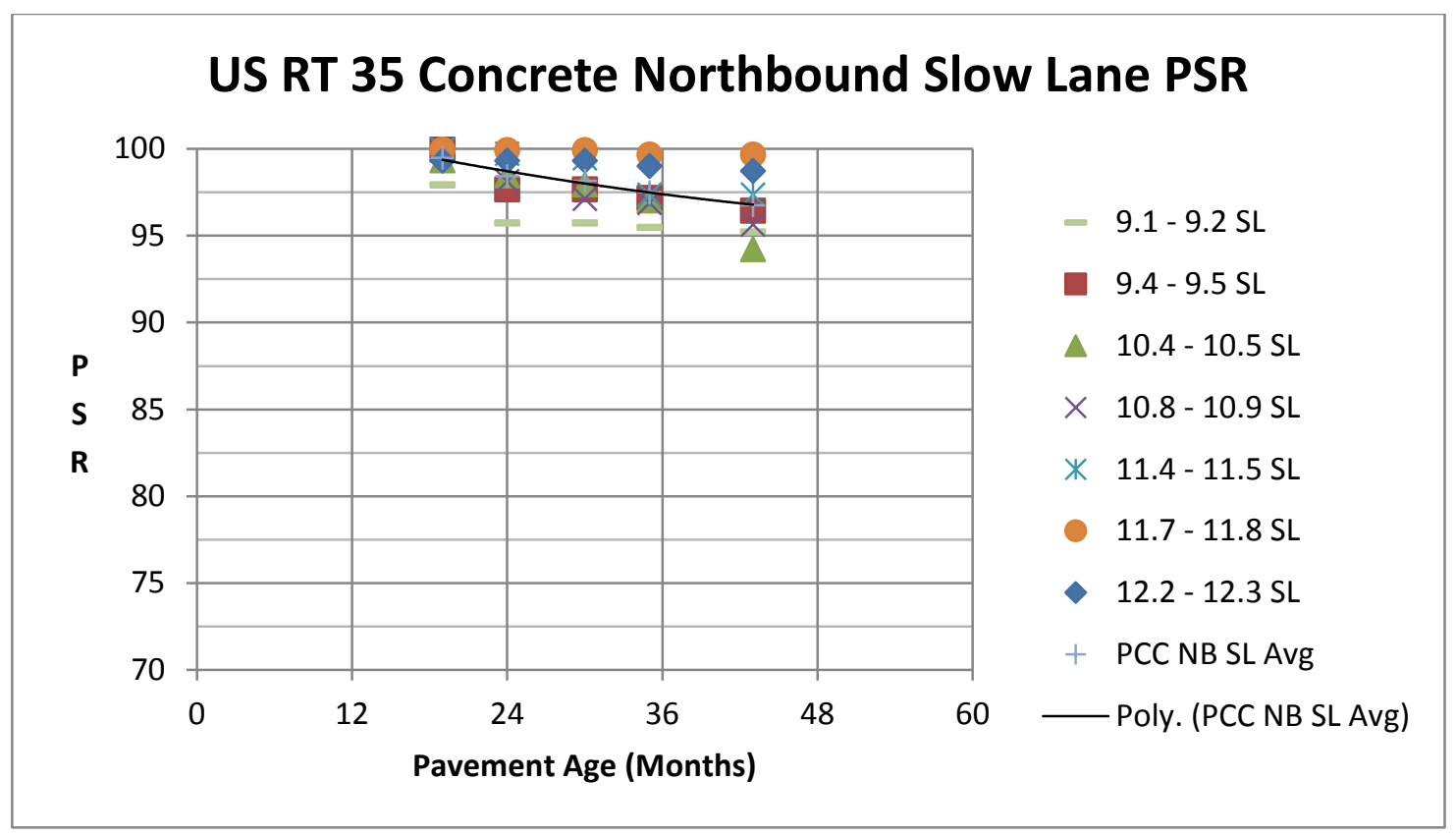

Figure 28: US 35 Concrete Northbound Slow Lane Pavement Age vs. PSR

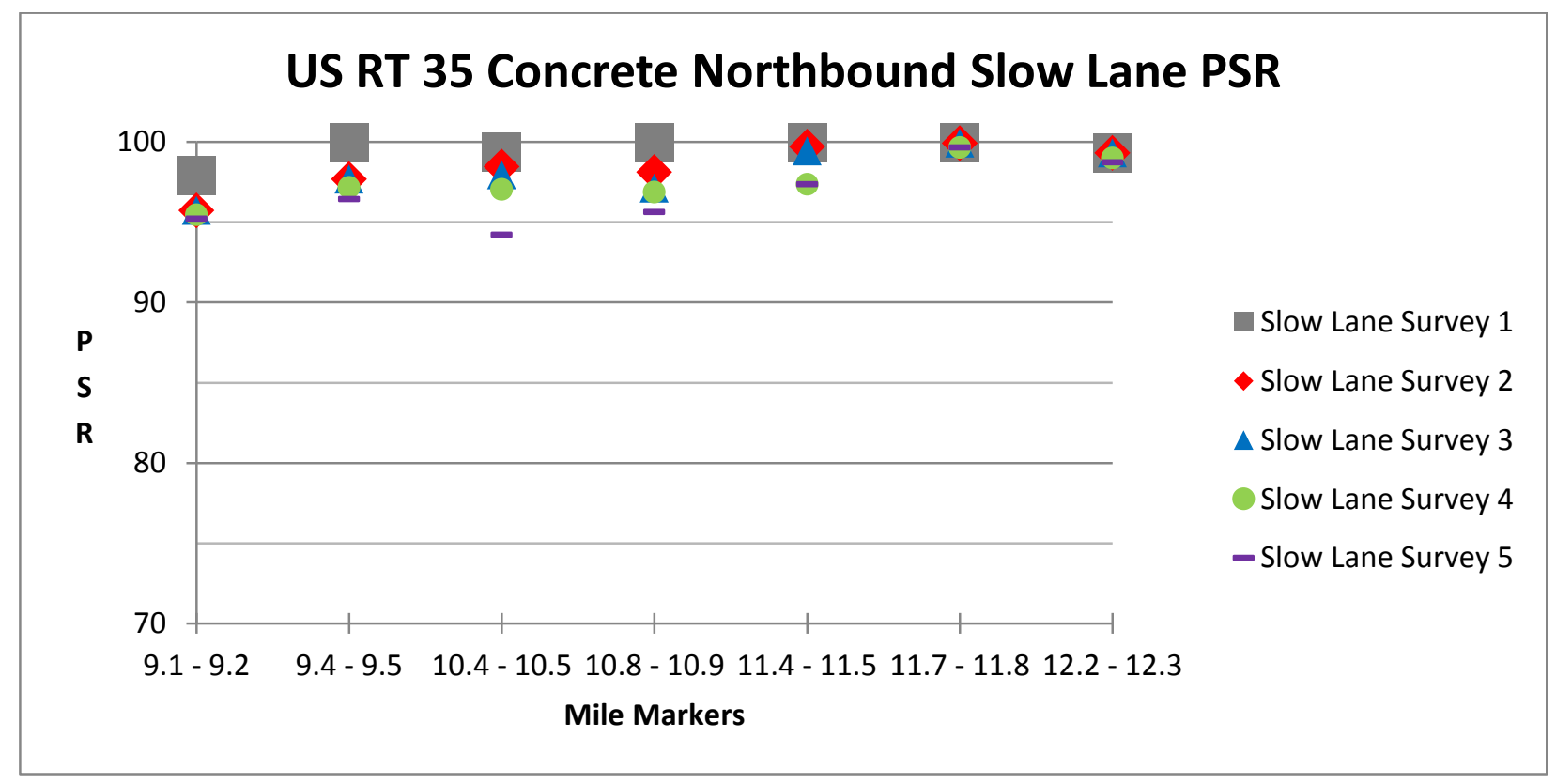

Figure 29: US 35 Concrete Northbound Slow Lane Mile Markers vs. PSR 


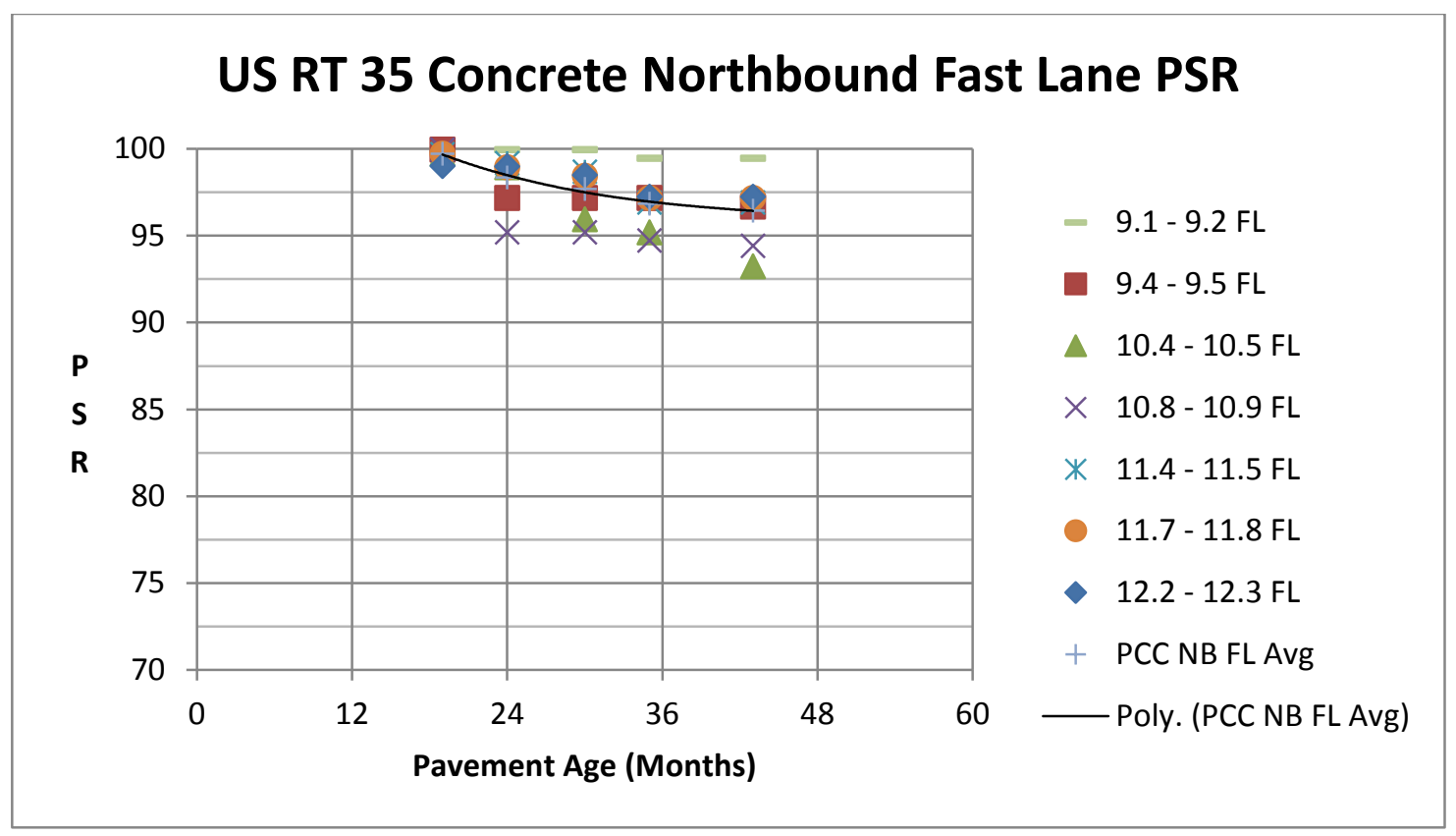

Figure 30: US 35 Concrete Northbound Fast Lane Pavement Age vs. PSR

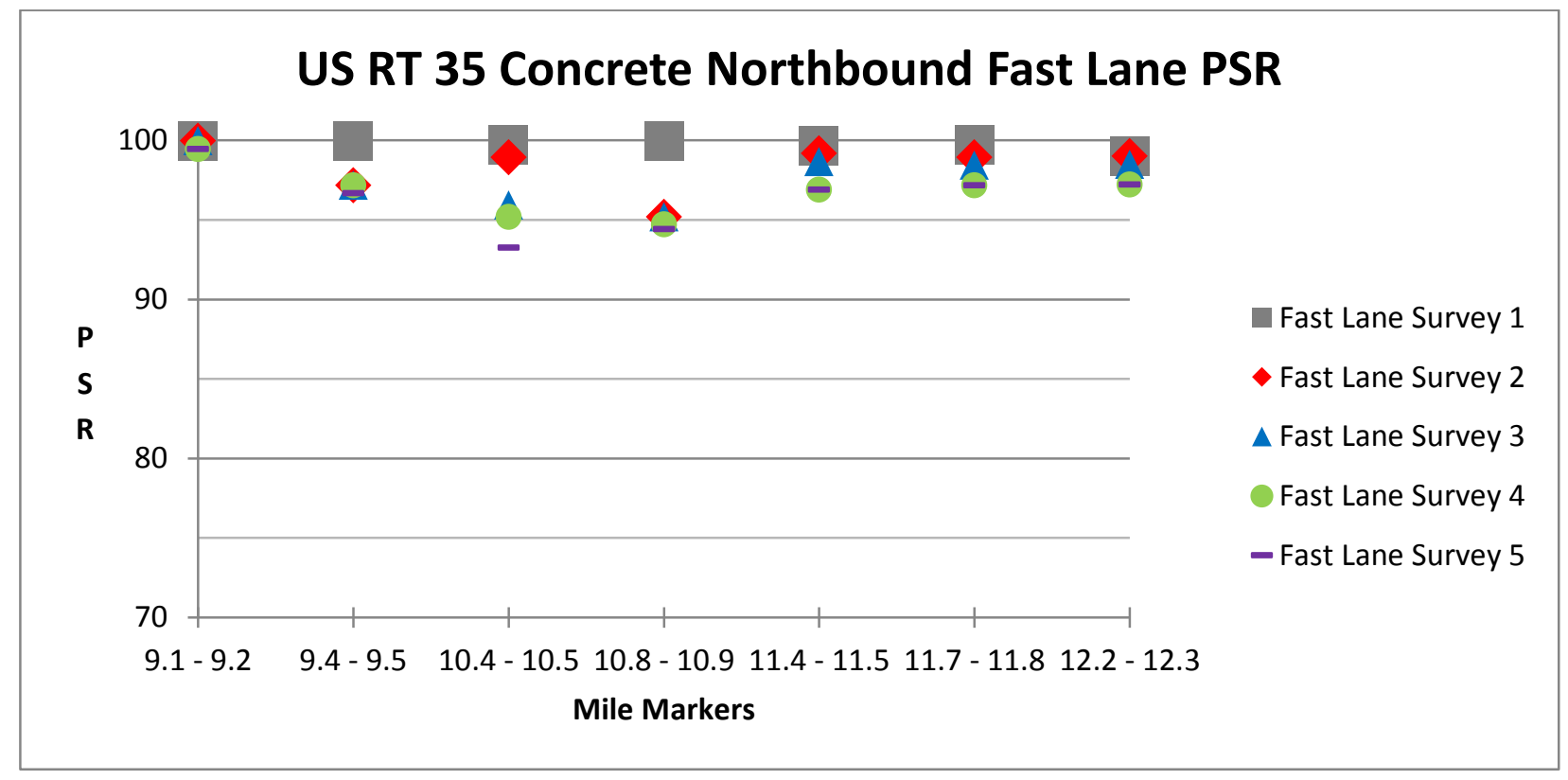

Figure 31: US 35 Concrete Northbound Fast Lane Mile Marker vs. PSR 


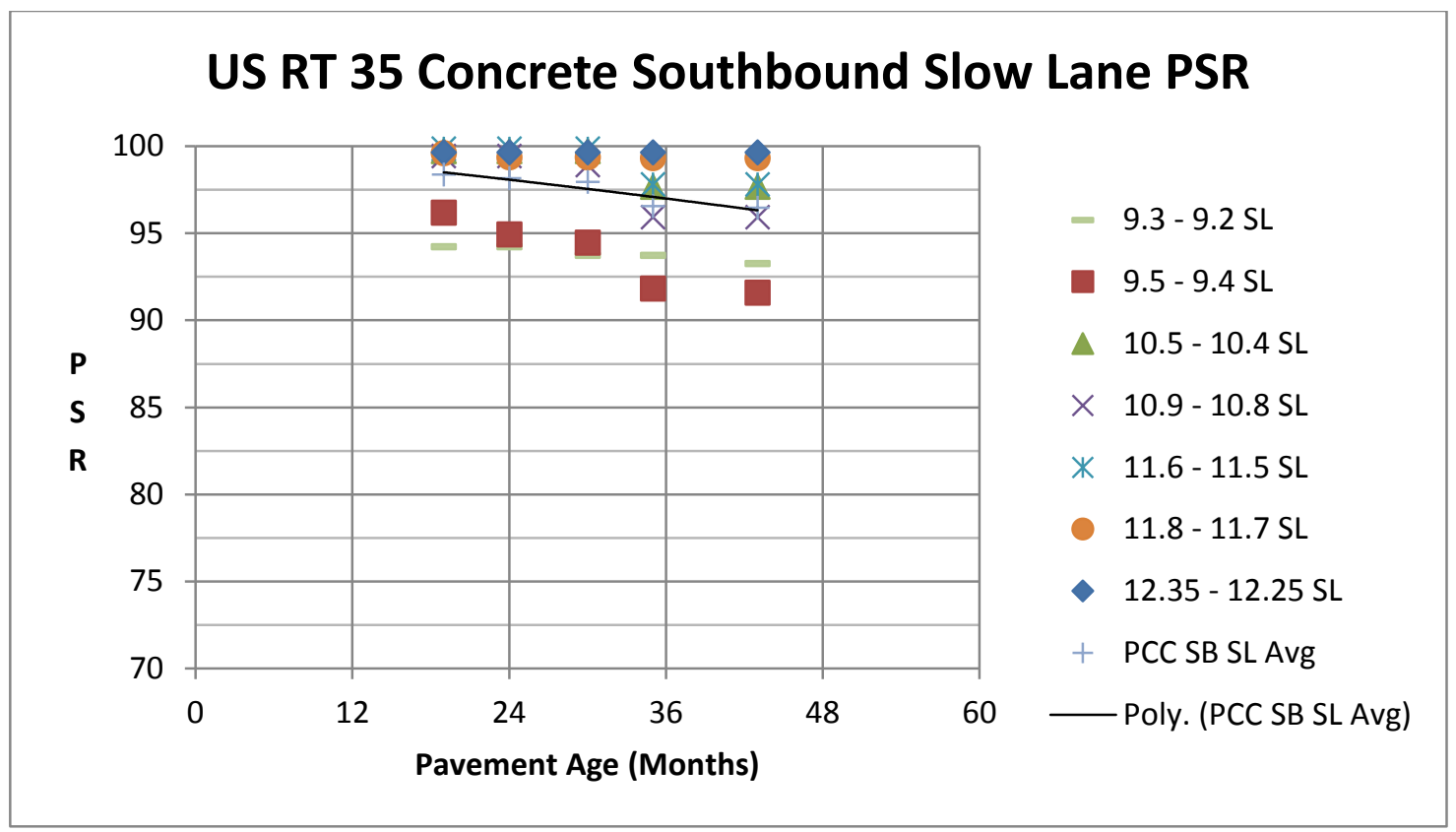

Figure 32: US 35 Concrete Southbound Slow Lane Pavement Age vs. PSR

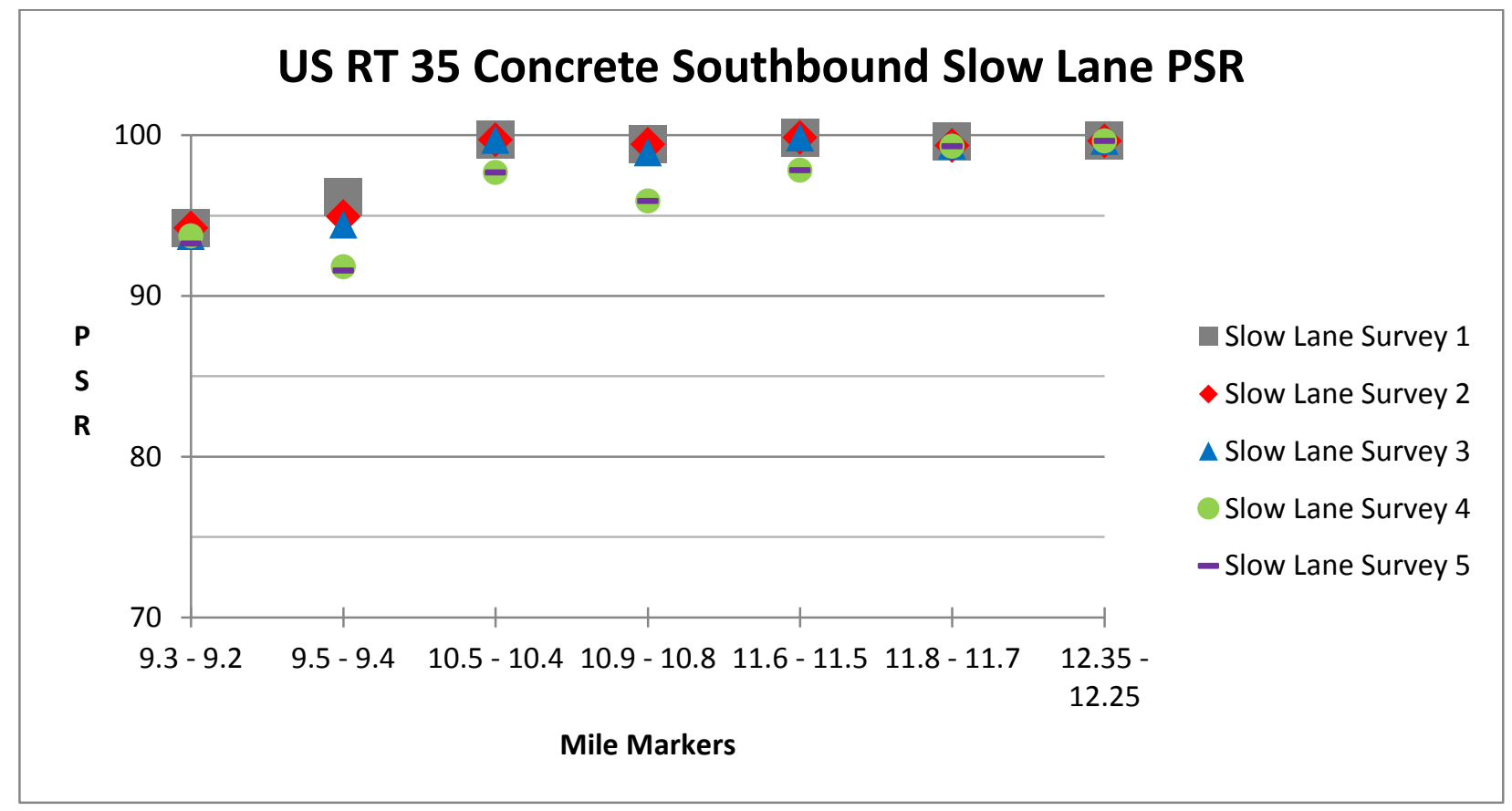

Figure 33: US 35 Concrete Southbound Slow Lane Mile Marker vs. PSR 


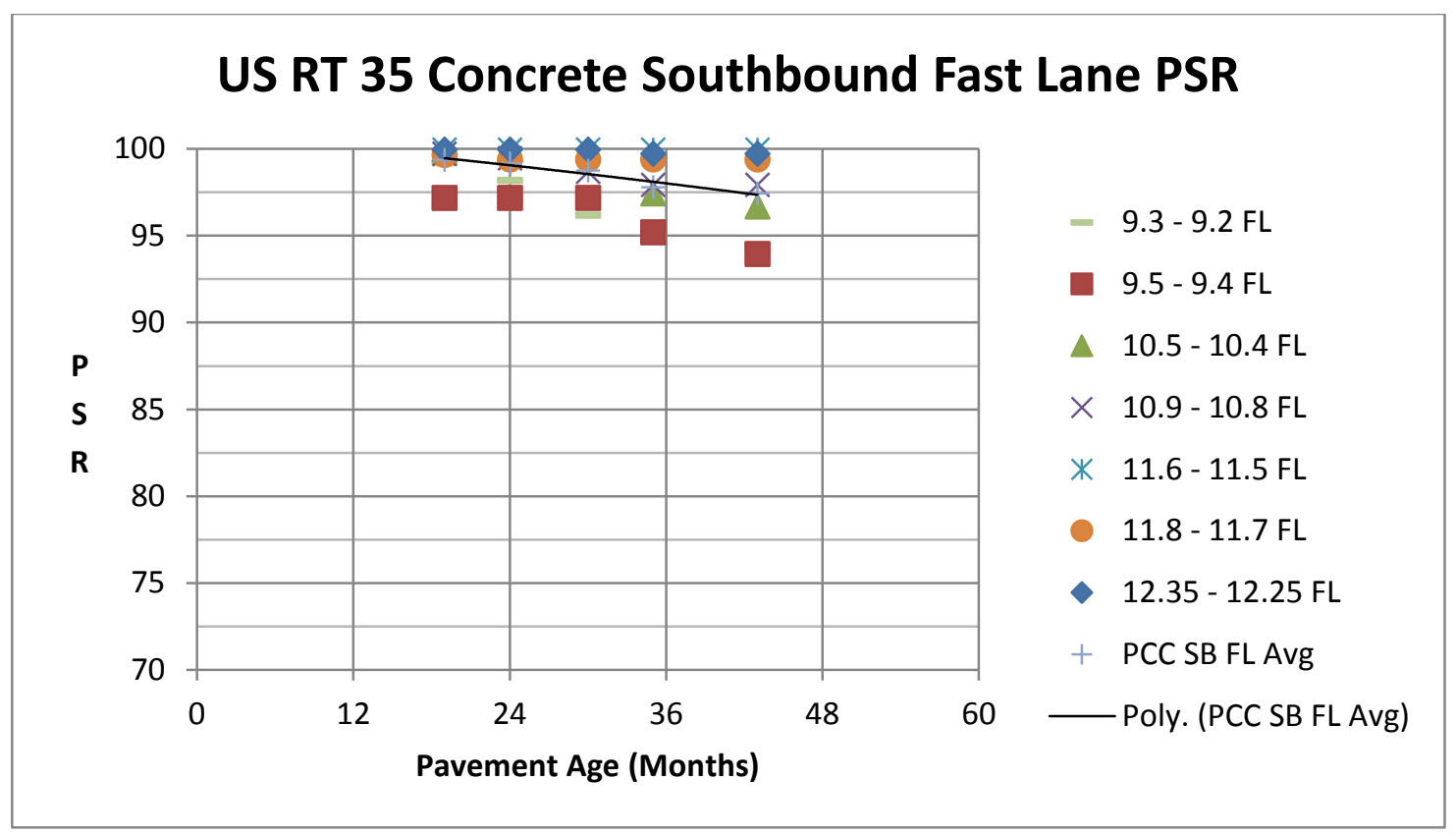

Figure 34: US 35 Concrete Southbound Fast Lane Pavement Age vs. PSR

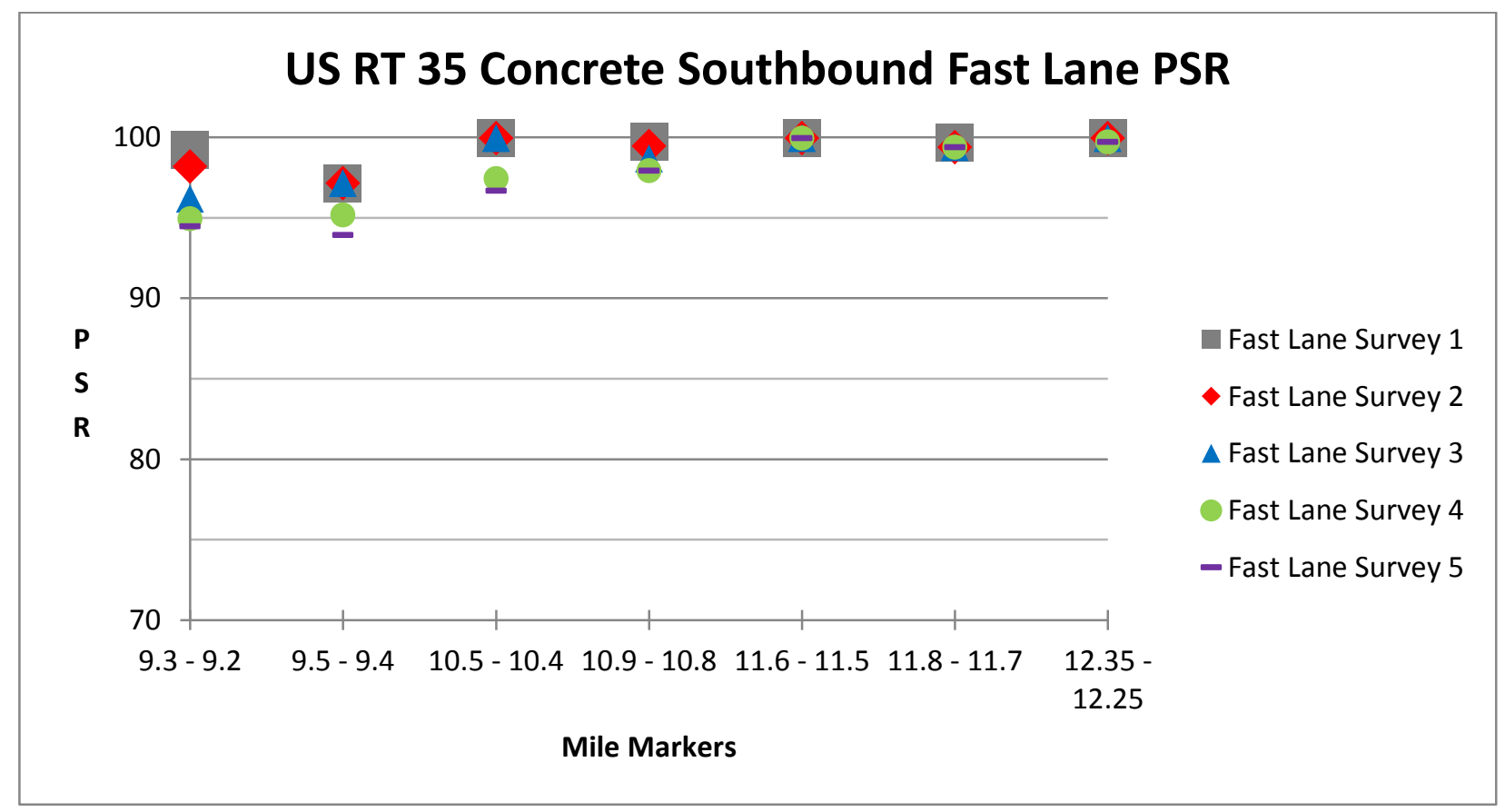

Figure 35: US 35 Concrete Southbound Fast Lane Mile Marker vs. PSR

\subsubsection{US 35 PSR Final Calculation vs. Original 2012 Spec. Calculation}

A comparison summary of PSR from survey 5 as calculated using the initial PSR calculation method and the final method is contained within Table 32 through Table 35 and Figure 36 through 
Figure 43. "2012 PSR" refers to PSR calculated using the April 2012 version of "West Virginia Department of Transportation Division of Highways Special Provision, Section 490: Nine Year Pavement Performance Criteria". "2015 PSR" refers to the final PSR calculation model described in the next section.

\begin{tabular}{|c|c|c|c|c|}
\cline { 2 - 5 } \multicolumn{1}{c|}{} & \multicolumn{2}{c|}{ Slow Lane } & \multicolumn{2}{c|}{ Fast Lane } \\
\hline Mile Post & $\begin{array}{c}2012 \\
\text { Spec. PSR }\end{array}$ & $\begin{array}{c}2015 \text { Spec. } \\
\text { PSR }\end{array}$ & $\begin{array}{c}2012 \\
\text { Spec. PSR }\end{array}$ & $\begin{array}{c}2015 \text { Spec. } \\
\text { PSR }\end{array}$ \\
\hline Average & 67.3 & 87.0 & 87.4 & 94.1 \\
\hline $12.6-12.7$ & 69.7 & 85.4 & 83.5 & 91.4 \\
\hline $13.2-13.3$ & 79.2 & 91.4 & 90.9 & 92.3 \\
\hline $13.8-13.9$ & 69.7 & 89.3 & 90.4 & 96.8 \\
\hline $14.4-14.5$ & 69.7 & 95.3 & 91.4 & 100.0 \\
\hline $14.6-14.7$ & 69.7 & 92.2 & 91.4 & 100.0 \\
\hline $15.2-15.3$ & 69.7 & 86.2 & 83.2 & 88.6 \\
\hline $15.4-15.5$ & 48.9 & 74.7 & 84.6 & 89.4 \\
\hline $16.0-16.1$ & 59.0 & 83.7 & 80.0 & 88.8 \\
\hline $16.4-16.5$ & 69.7 & 84.4 & 91.4 & 100.0 \\
\hline
\end{tabular}

Table 32: Asphalt Northbound Survey 5 - 2012 Spec. vs 2015 Spec.

\begin{tabular}{|c|c|c|c|c|}
\cline { 2 - 5 } \multicolumn{1}{c|}{} & \multicolumn{2}{c|}{ Slow Lane } & \multicolumn{2}{c|}{ Fast Lane } \\
\hline Mile Post & $\begin{array}{c}2012 \\
\text { Spec. PSR }\end{array}$ & $\begin{array}{c}2015 \text { Spec. } \\
\text { PSR }\end{array}$ & $\begin{array}{c}2012 \\
\text { Spec. PSR }\end{array}$ & $\begin{array}{c}2015 \text { Spec. } \\
\text { PSR }\end{array}$ \\
\hline Average & 69.78 & 89.1 & 78.04 & 93.4 \\
\hline $12.7-12.6$ & 69.75 & 88.4 & 78.43 & 97.6 \\
\hline $13.3-13.2$ & 55.77 & 81.4 & 45.95 & 87.3 \\
\hline $13.9-13.8$ & 69.75 & 90.2 & 69.75 & 83.5 \\
\hline $14.5-14.4$ & 69.99 & 89.5 & 82.09 & 87.1 \\
\hline $14.7-14.6$ & 69.95 & 94.9 & 76.19 & 100.0 \\
\hline $15.3-15.2$ & 67.18 & 85.6 & 78.98 & 90.2 \\
\hline $15.5-15.4$ & 69.75 & 85.8 & 88.68 & 95.3 \\
\hline $16.1-16.0$ & 85.53 & 97.1 & 90.90 & 100.0 \\
\hline $16.5-16.4$ & 70.32 & 89.5 & 91.37 & 100.0 \\
\hline
\end{tabular}

Table 33: Asphalt Southbound Survey 5 - 2012 Spec. vs 2015 Spec. 


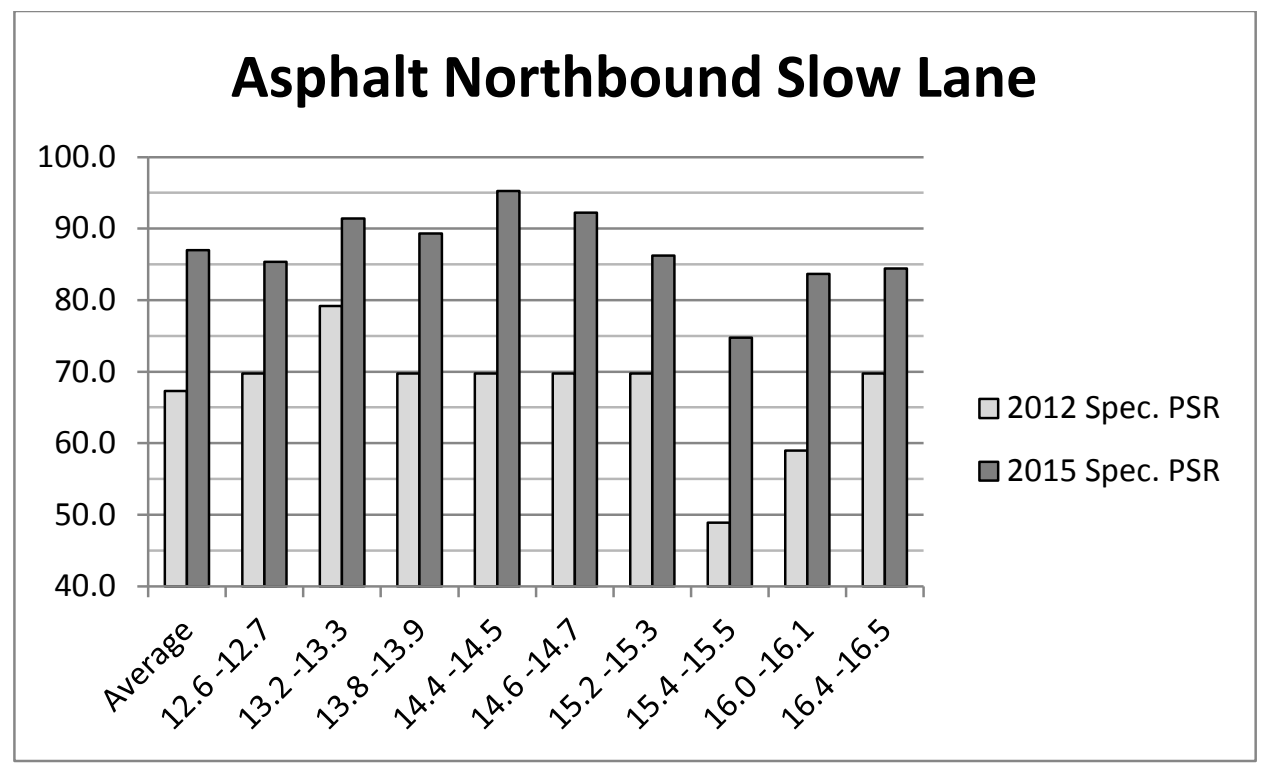

Figure 36: Asphalt Northbound Slow Lane Survey 5 - 2012 Spec. vs 2015 Spec.

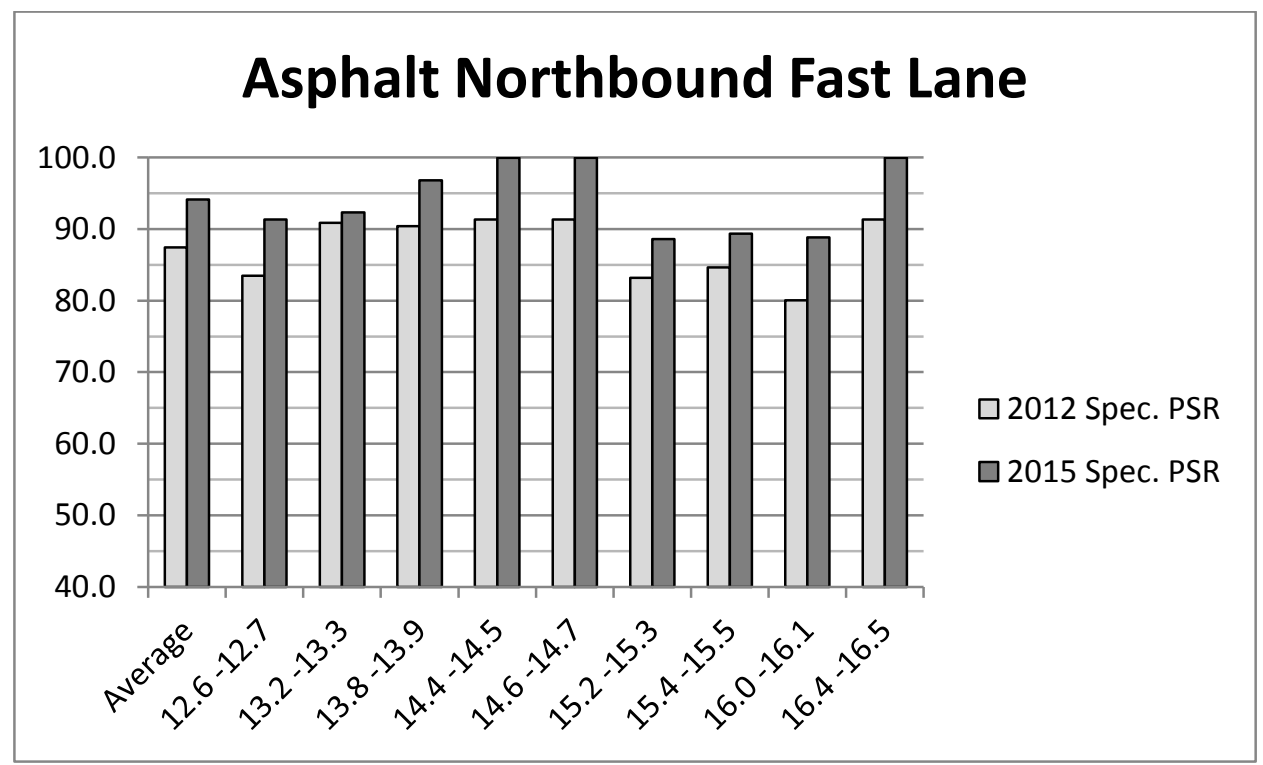

Figure 37: Asphalt Northbound Fast Lane Survey 5 - 2012 Spec. vs 2015 Spec. 


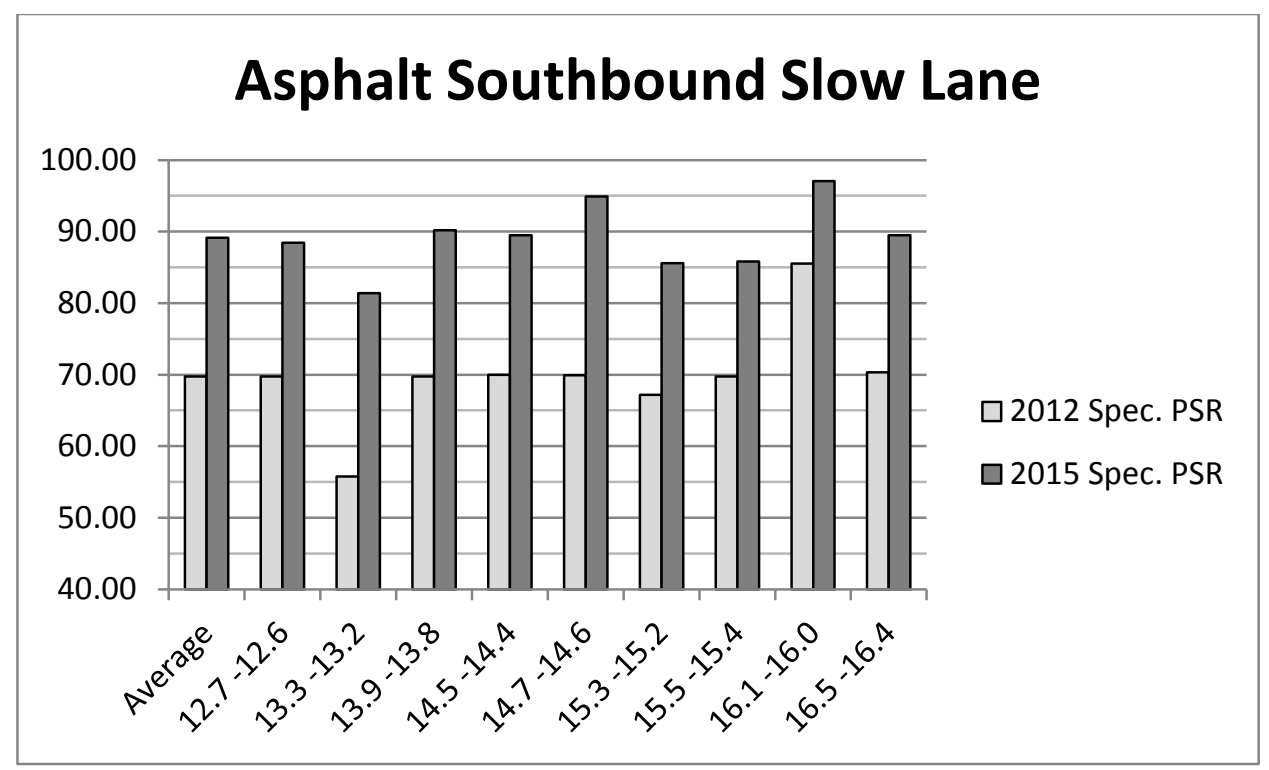

Figure 38: Asphalt Southbound Slow Lane Survey 5 - 2012 Spec. vs 2015 Spec.

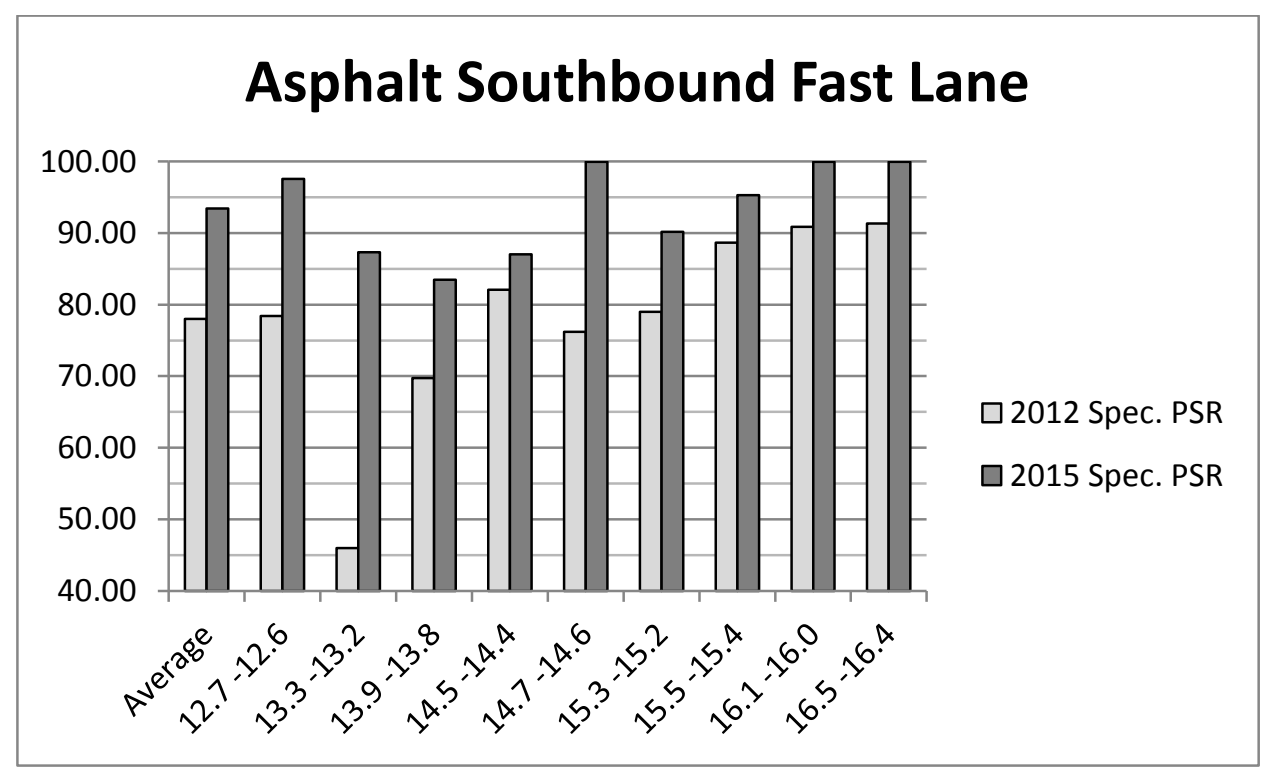

Figure 39: Asphalt Southbound Fast Lane Survey 5 - 2012 Spec. vs 2015 Spec. 


\begin{tabular}{|c|c|c|c|c|}
\cline { 2 - 5 } \multicolumn{1}{c|}{} & \multicolumn{2}{c|}{ Slow Lane } & \multicolumn{2}{c|}{ Fast Lane } \\
\hline Mile Post & $\begin{array}{c}2012 \text { Spec. } \\
\text { PSR }\end{array}$ & $\begin{array}{c}2015 \text { Spec. } \\
\text { PSR }\end{array}$ & $\begin{array}{c}2012 \text { Spec. } \\
\text { PSR }\end{array}$ & $\begin{array}{c}2015 \text { Spec. } \\
\text { PSR }\end{array}$ \\
\hline Average & 79.23 & 96.76 & 84.50 & 96.45 \\
\hline $9.1-9.2$ & 79.32 & 95.23 & 94.96 & 99.46 \\
\hline $9.4-9.5$ & 75.53 & 96.44 & 80.14 & 96.68 \\
\hline $10.4-10.5$ & 65.74 & 94.22 & 77.10 & 93.25 \\
\hline $10.8-10.9$ & 64.74 & 95.65 & 75.34 & 94.42 \\
\hline $11.4-11.5$ & 84.37 & 97.37 & 86.33 & 96.92 \\
\hline $11.7-11.8$ & 96.19 & 99.67 & 87.91 & 97.18 \\
\hline $12.2-12.3$ & 88.70 & 98.73 & 89.74 & 97.24 \\
\hline
\end{tabular}

Table 34: Concrete Northbound Survey 5 - 2012 Spec. vs 2015 Spec.

\begin{tabular}{|c|c|c|c|c|}
\cline { 2 - 5 } \multicolumn{1}{c|}{} & \multicolumn{2}{c|}{ Slow Lane } & \multicolumn{2}{c|}{ Fast Lane } \\
\hline Mile Post & $\begin{array}{c}2012 \text { Spec. } \\
\text { PSR }\end{array}$ & $\begin{array}{c}2015 \text { Spec. } \\
\text { PSR }\end{array}$ & $\begin{array}{c}2012 \text { Spec. } \\
\text { PSR }\end{array}$ & $\begin{array}{c}2015 \text { Spec. } \\
\text { PSR }\end{array}$ \\
\hline Average & 82.67 & 96.47 & 89.85 & 97.44 \\
\hline $9.3-9.2$ & 71.20 & 93.27 & 83.72 & 94.47 \\
\hline $9.5-9.4$ & 75.73 & 91.59 & 77.90 & 93.95 \\
\hline $10.5-10.4$ & 70.47 & 97.68 & 87.91 & 96.68 \\
\hline $10.9-10.8$ & 85.02 & 95.92 & 91.84 & 97.94 \\
\hline $11.5-11.4$ & 89.91 & 97.82 & 97.68 & 99.97 \\
\hline $11.8-11.7$ & 92.71 & 99.33 & 96.62 & 99.39 \\
\hline $12.35-12.25$ & 93.63 & 99.65 & 93.27 & 99.71 \\
\hline
\end{tabular}

Table 35: Concrete Southbound - Survey 5 - 2012 Spec. vs 2015 Spec.

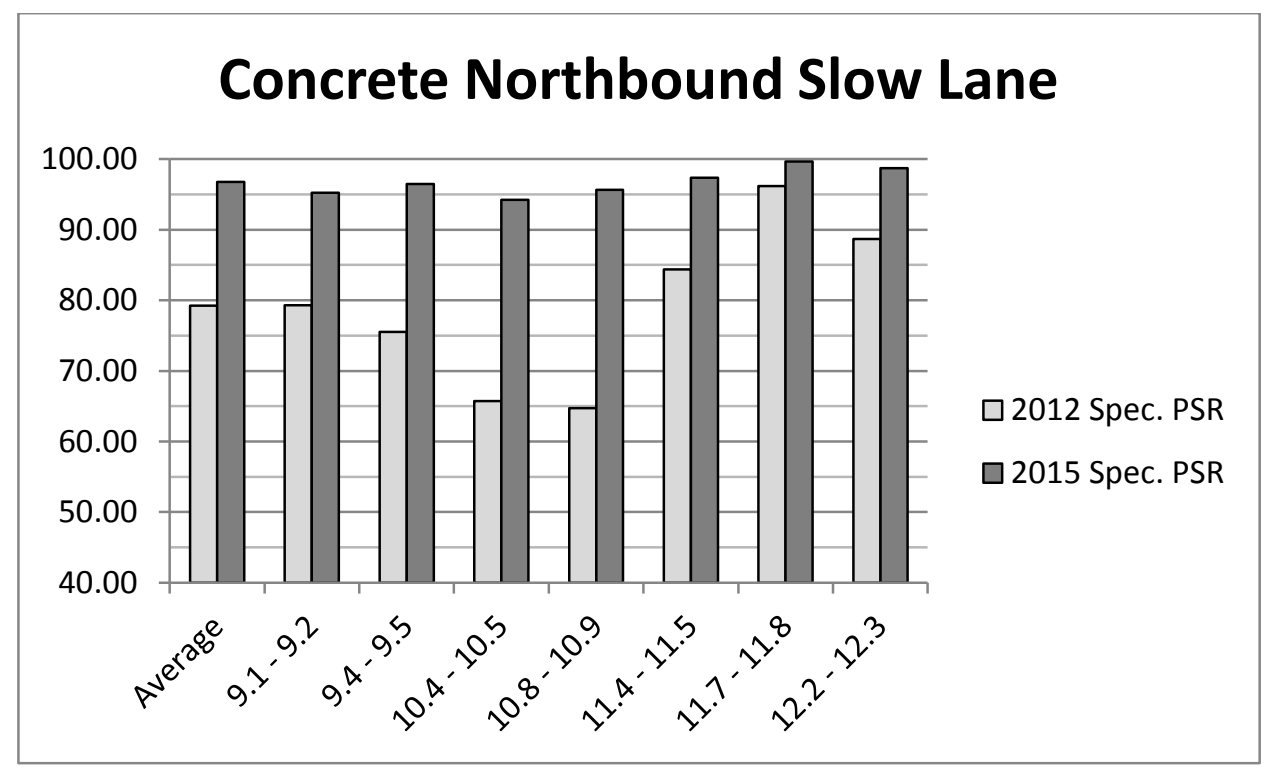

Figure 40: Concrete Northbound Slow Lane Survey 5 - 2012 Spec. vs 2015 Spec. 


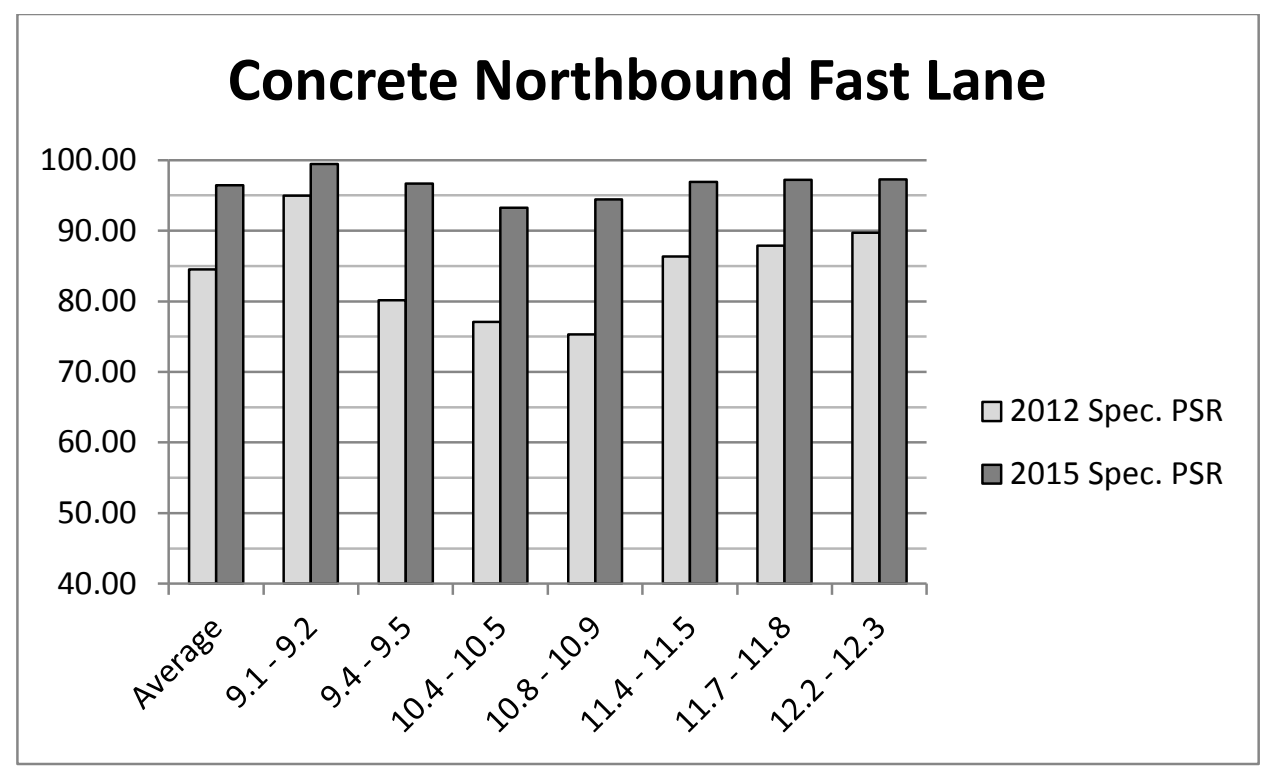

Figure 41: Concrete Northbound Fast Lane Survey 5 - 2012 Spec. vs 2015 Spec.

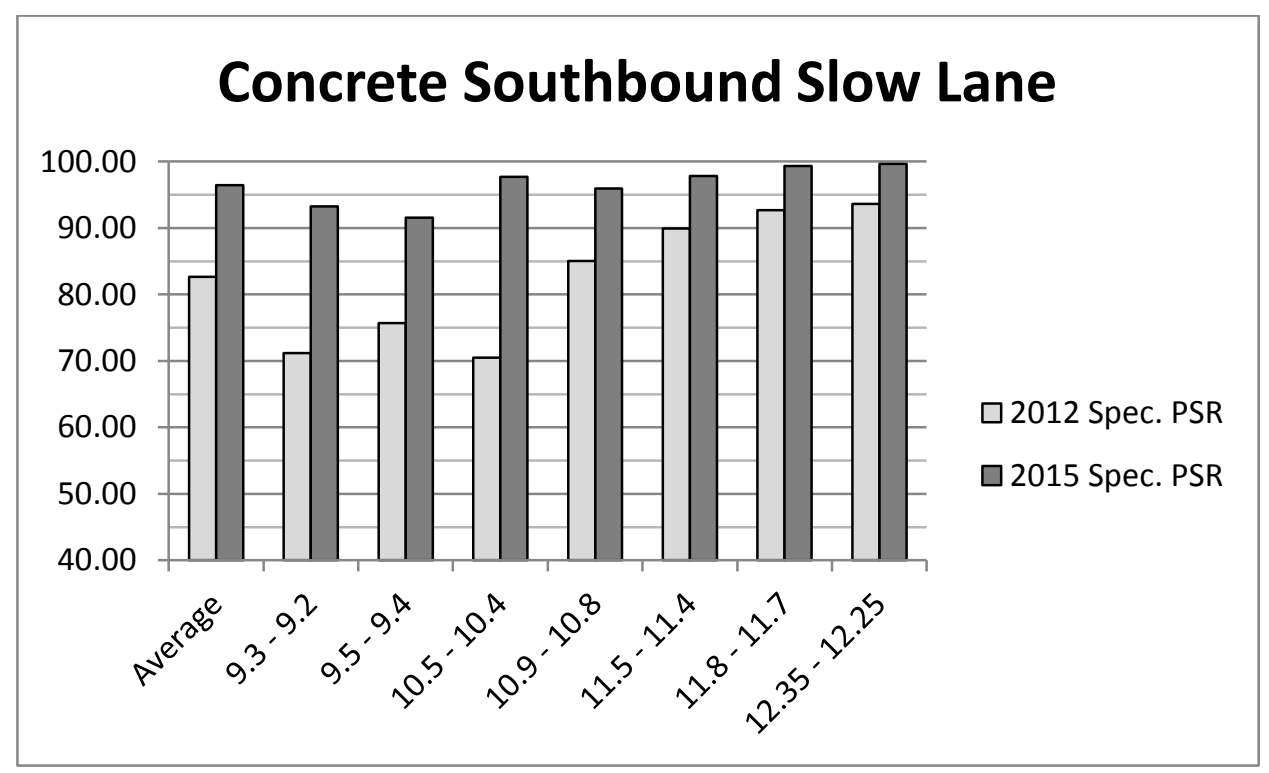

Figure 42: Concrete Southbound Slow Lane Survey 5 - 2012 Spec. vs 2015 Spec. 


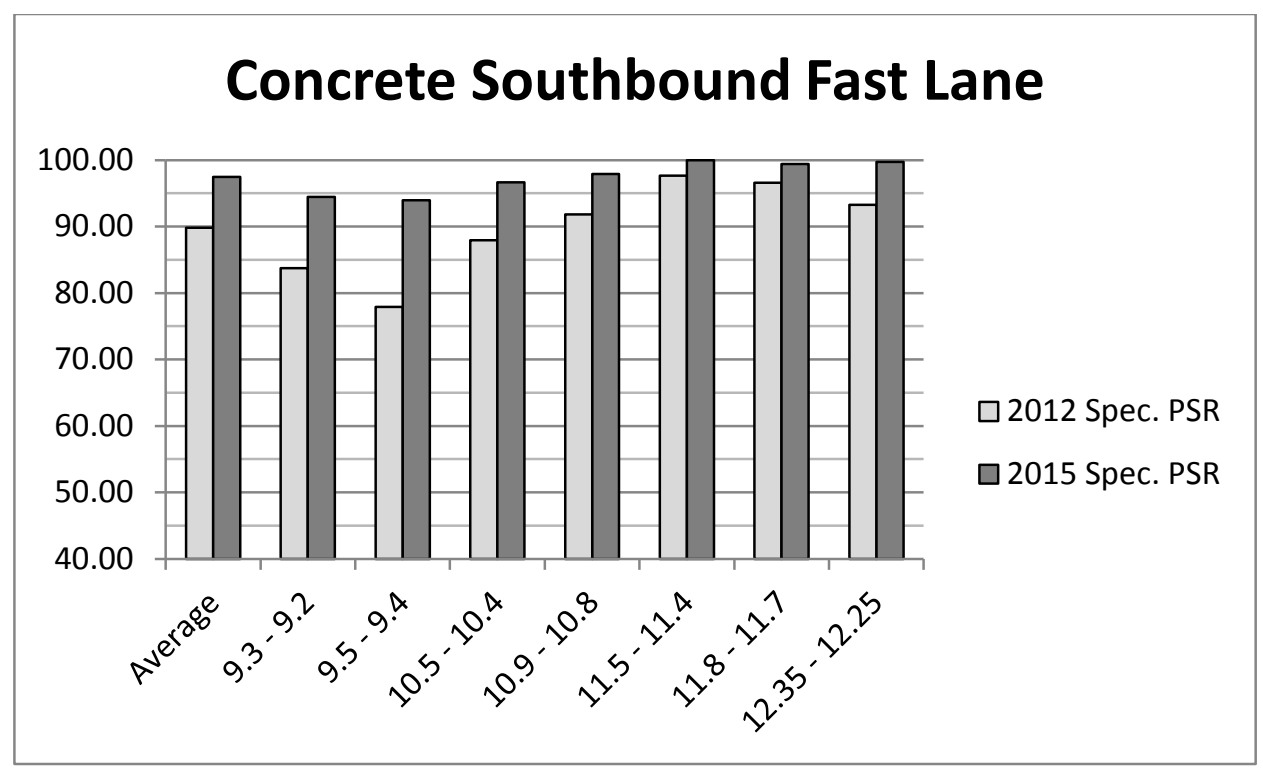

Figure 43: Concrete Southbound Fast Lane Survey 5 - 2012 Spec. vs. 2015 Spec.

\subsection{Comparison of ARAN and PSR Survey Surface Distress Data}

The comparisons between indices provided and indices calculated for the first three surveys are shown in Figure 44 through Figure 49. Within the graph legends, "S.P." stands for Special Provision which simply identifies the indices generated with the distress data manually collected for the calculation of Section 490, Special Provision PSR. "PMS data" is the index data provided by the previously mentioned ARAN, and dTIMS software. The "PMS data" were collected shortly after the second survey was completed.

$\mathrm{SCl}, \mathrm{ECl}, \mathrm{NCl}$, and $\mathrm{RDI}$ comparisons were made for the northbound slow lane asphalt surface sections and $\mathrm{JCl}$ and CSI for northbound slow lane asphalt surface sections within US 35 . The horizontal axis of each figure corresponds to the applicable test section stations for which comparisons could be made. A single index value is associated with each test section. The data points have been connected simply to make the identification of points easier as indices in many test sections overlap. It should not be assumed that index values between test sections are reflective of these lines. 


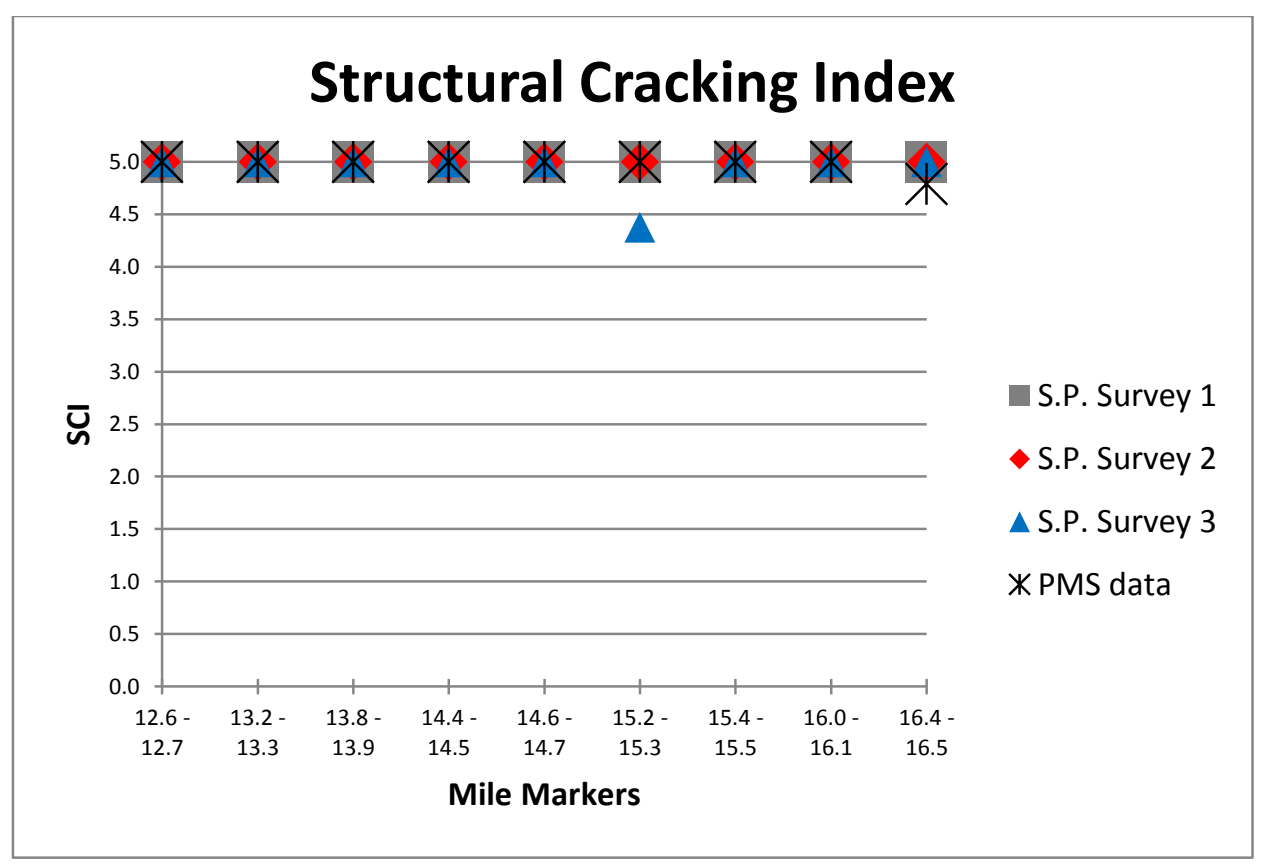

Figure 44: Structural Cracking Index - Manual Survey Data vs. PMS Data Comparison

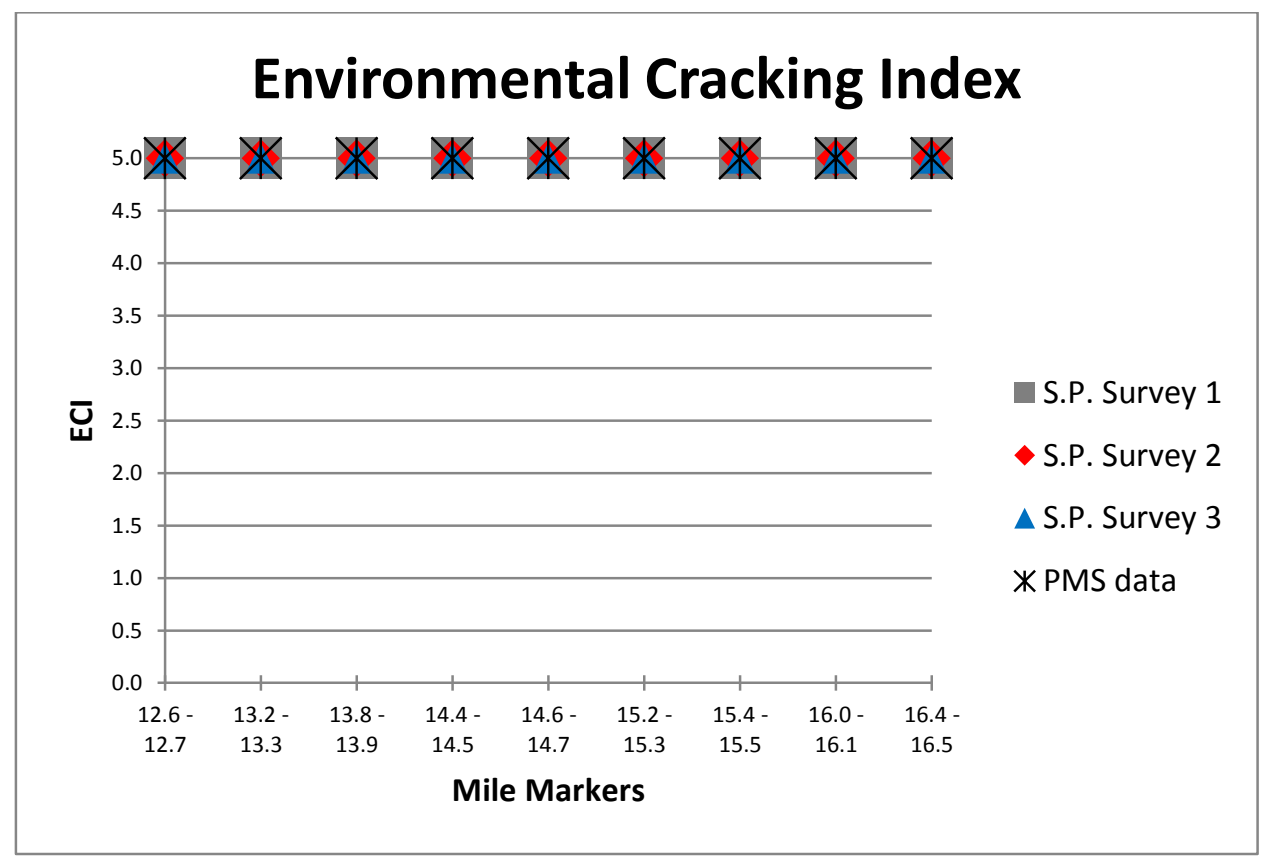

Figure 45: Environmental Cracking Index - Manual Survey Data vs. PMS Data Comparison 


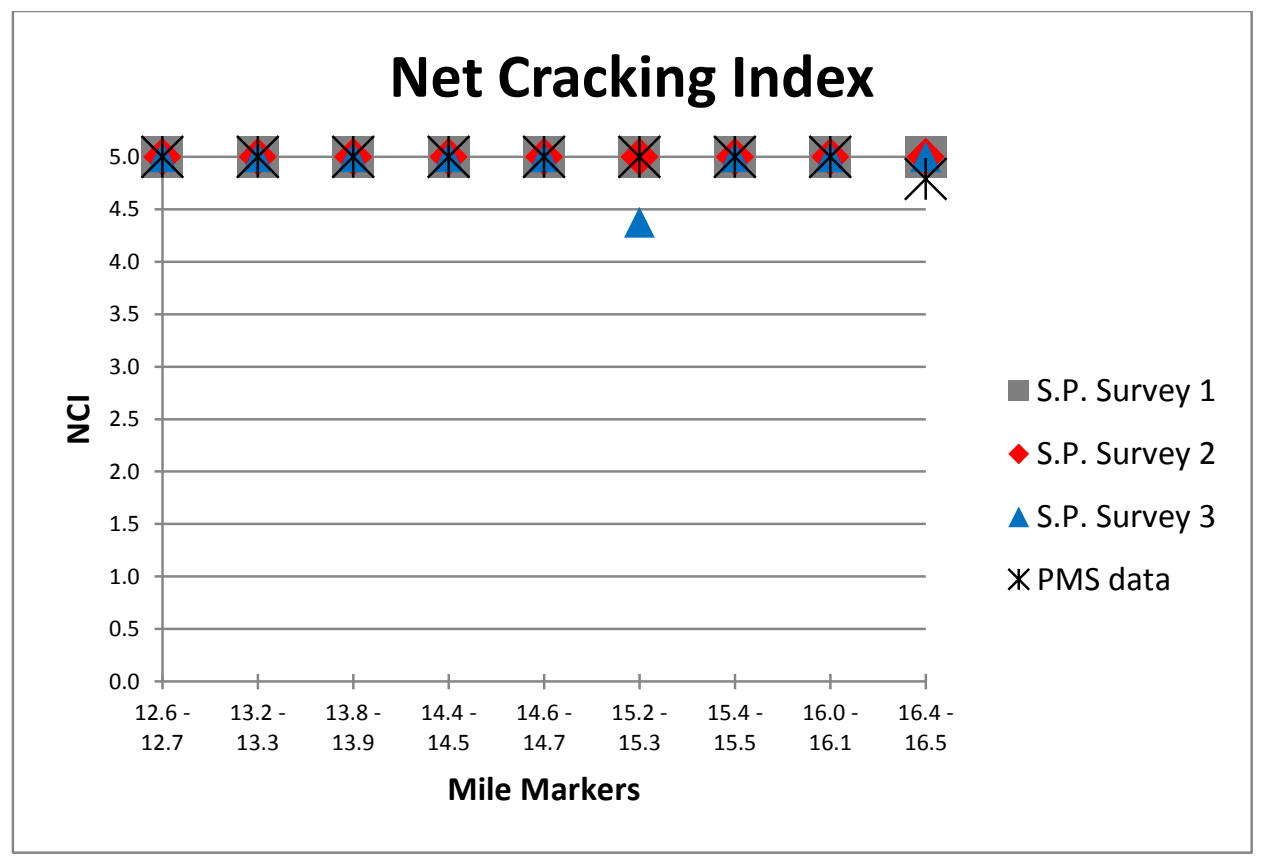

Figure 46: Net Cracking Index - Manual Survey Data vs. PMS Data Comparison

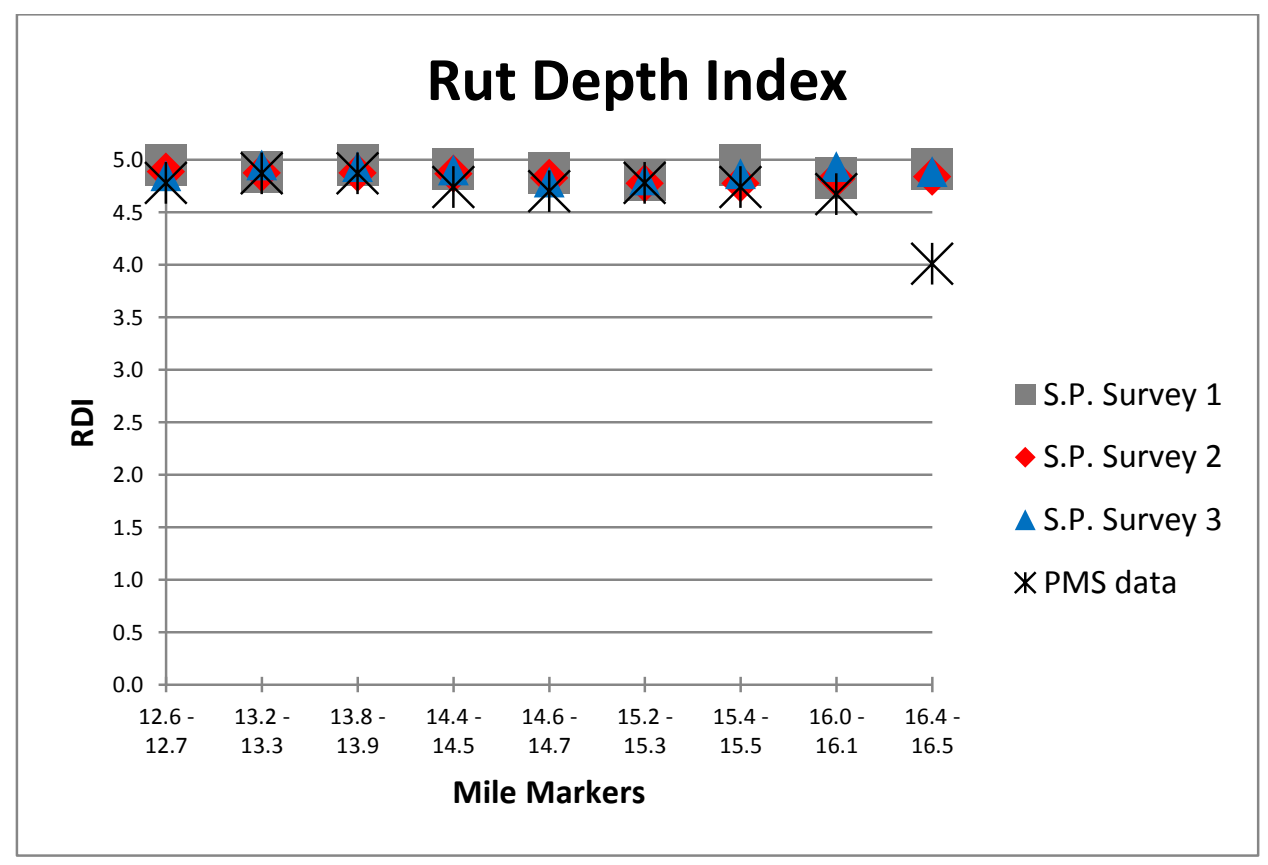

Figure 47: Rut Depth Index - Manual Survey Data vs. PMS Data Comparison 


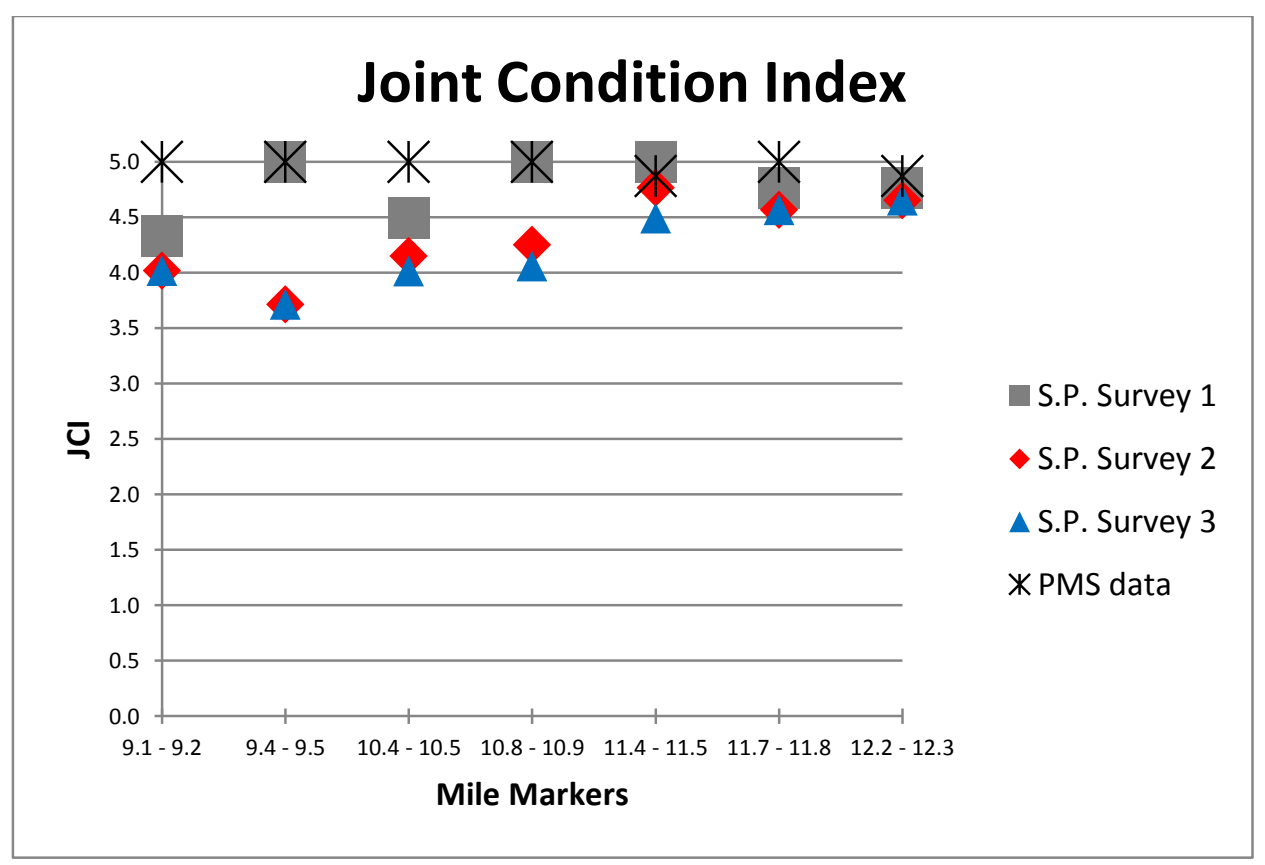

Figure 48: Joint Condition Index - Manual Survey Data vs. PMS Data Comparison

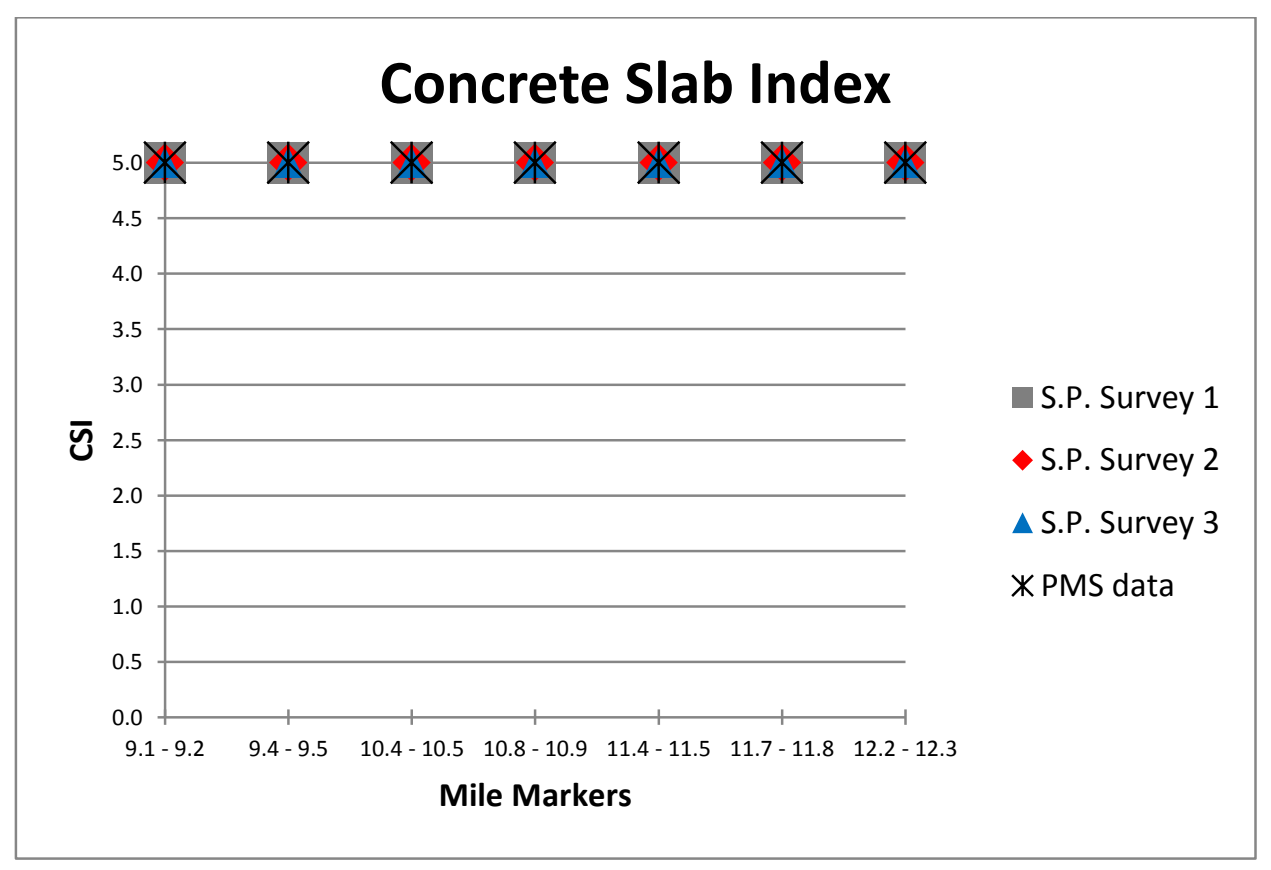

Figure 49: Concrete Slab Index - Manual Survey Data vs. PMS Data Comparison 


\subsection{Deflection Data Results and Summary}

As was stated within Chapter 3 and Chapter $4, \mathrm{SN}_{\text {eff }}$ was used for comparing analysis methods for asphalt pavements. After techniques were removed in which $\mathrm{M}_{\mathrm{R}^{*}}$ was used in calculations, the resulting final averaged asphalt $\mathrm{SN}_{\text {eff }}$ values are shown in Table 36 and Figure 50.

\begin{tabular}{|c|c|c|c|c|c|c|}
\hline & $\begin{array}{l}S N_{\text {eff }} \text { as a function of } \\
E_{p} \text { as a function of } \\
M_{R} \text { from a } 3 \text { layer } \\
\text { MODULUS } 6.1 \\
\text { backcalculation }\end{array}$ & $\begin{array}{l}\mathrm{SN}_{\text {eff }} \text { as a function of } \\
\mathrm{E}_{\mathrm{p}} \text { from a } 2 \text { layer } \\
\text { MODULUS } 6.1 \\
\text { backcalculation }\end{array}$ & $\begin{array}{l}S N_{\text {eff }} \text { as a function of } \\
E_{p} \text { and } M_{R} \text { from a } 2 \\
\text { layer MODULUS } 6.1 \\
\text { backcalculation }\end{array}$ & $\begin{array}{l}\mathrm{SN}_{\text {eff }} \text { as provided by } \\
3 \text { layer MODTAG } \\
\text { backcalculation }\end{array}$ & $\begin{array}{l}\mathrm{SN}_{\text {eff }} \text { as a function of } \\
\mathrm{E}_{\mathrm{p}} \text { and } \mathrm{M}_{\mathrm{R}} \text { from a } 3 \\
\text { layer MODTAG } \\
\text { backcalculation }\end{array}$ & $\begin{array}{l}S N_{\text {eff }} \text { as a function of } \\
E_{p} \text { and } M_{R} \text { from a } 3 \\
\text { layer BACKFAA } \\
\text { backcalculation }\end{array}$ \\
\hline Program & MODULUS 6.1 & MODULUS 6.1 & MODULUS 6.1 & MODTAG & MODTAG & BACKFAA \\
\hline Backcalc using: & 3 Layer & 2 Layer & 2 Layer & 3 Layer & 3 Layer & 3 Layer \\
\hline $\mathrm{E}_{\mathrm{p}}$ calculated with: & $M_{R}$ & ( $E_{p}$ provided) & $M_{R}$ & not calculated & $M_{R}$ & $M_{R}$ \\
\hline NB HMA Avg $\mathrm{SN}_{\text {eff }}$ & 4.65 & 5.30 & 4.71 & 4.31 & 4.90 & 5.25 \\
\hline SB HMA Avg $\mathrm{SN}_{\mathrm{eff}}$ & 4.19 & 4.76 & 4.24 & 4.56 & 4.42 & 4.53 \\
\hline All HMA Avg $\mathrm{SN}_{\mathrm{eff}}$ & 4.42 & 5.03 & 4.48 & 4.44 & 4.66 & 4.89 \\
\hline
\end{tabular}

Table 36: Final Summary of Estimated Asphalt $\mathrm{SN}_{\mathrm{eff}}$

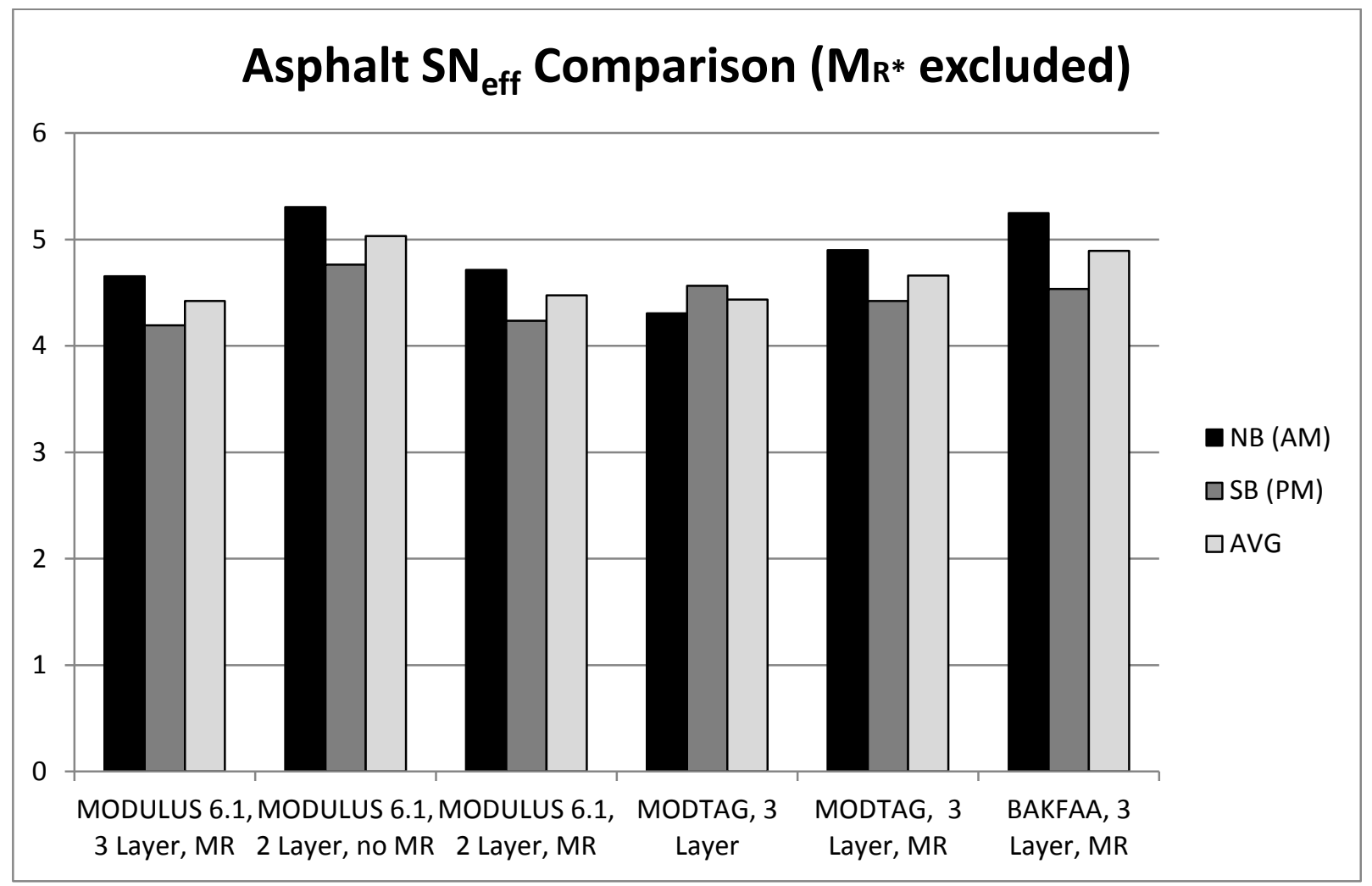

Figure 50: Asphalt $\mathrm{SN}_{\mathrm{eff}}$ Comparison 
One additional result which was observed pertained to the effect of temperature and temperature correction (or lack thereof). The BAKFAA backcalculation program included no input or correction for temperature so any effect of temperature on backcalculation should be most noticeable in this program. Figure 51 and Figure 52 show the resulting temperature vs. moduli graphs for surface and subgrade layers.

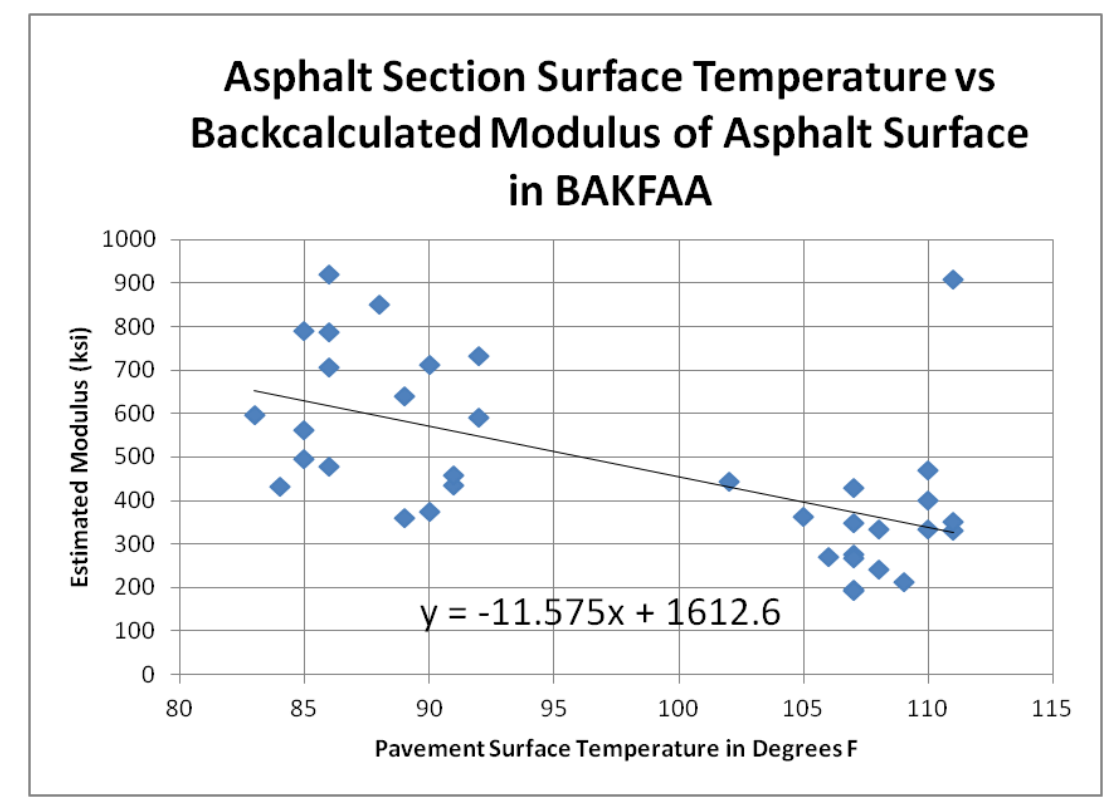

Figure 51: BAKFAA Temperature vs. Surface Modulus

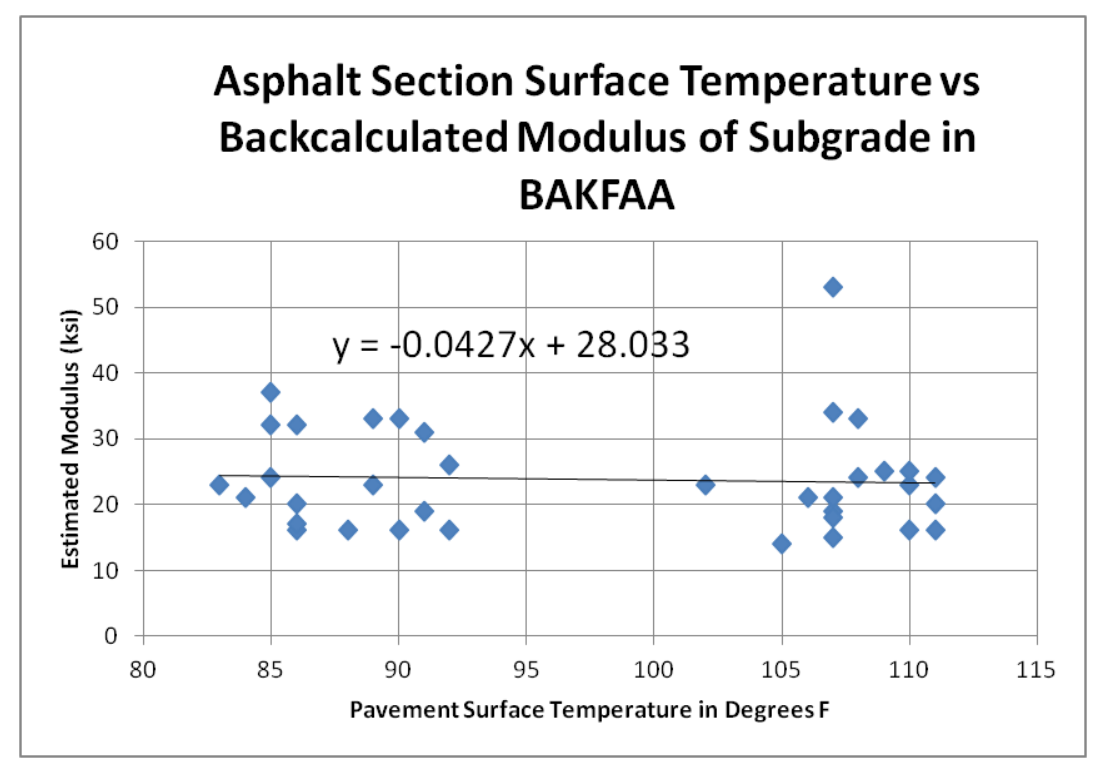

Figure 52: BAKFAA Temperature vs. Subgrade Modulus 
Table 37 shows all average subgrade moduli values backcalculated from asphalt and concrete deflection data. All three programs provided subgrade moduli values for the asphalt section tests. Due to the screening process and the limited number of eligible concrete tests only BAKFAA was able to perform backcalculations on the 34 concrete drops. Concrete section subgrade moduli backcalculated with BAKFAA were higher than asphalt section subgrade moduli from BAKFAA. Within the asphalt section, MODULUS 6.1 and MODTAG backcalculations produced subgrade moduli greater than BAKFAA in either section. All backcalculation programs determined that, on average, northbound subgrade moduli were greater than southbound subgrade moduli.

\begin{tabular}{|r|c|c|c|c|}
\cline { 2 - 5 } & \multicolumn{3}{|c|}{ Asphalt } & Concrete \\
\cline { 2 - 5 } North AVG & MODULUS 6.1 & MODTAG & BAKFAA & BAKFAA \\
\cline { 2 - 5 } South AVG & 33.2 & 32.5 & 24.2 & 26.6 \\
AVG & 30.7 & 25.7 & 23.6 & 25.0 \\
\cline { 2 - 5 } & 32.0 & 29.1 & 23.9 & 25.8 \\
\hline
\end{tabular}

Table 37: Asphalt and Concrete Average Subgrade Modulus Values 


\section{Chapter 6. Conclusions and Recommendations}

\subsection{Conclusions}

\subsubsection{Pavement Surface Rating}

The initial and intermediate PSR calculations for US 35 are now obsolete and are only included to mark the progression of changes made. No conclusions about pavement performance or condition should be made based on 2012 PSR methodology.

Testing the accuracy of the surveyors was not within the scope of this research. It was the decision of WVDOH authorities to use the same survey form each time. Distresses recorded on previous surveys likely influenced subsequent survey recordings, but the integrity of the system was maintained. On a few occasions, distresses were noted and then either disappeared or decreased in extent or severity on a subsequent survey. This could be due to inaccurate judgments or measurements on either survey, erroneous documentation, or could be completely legitimate as some distresses such as cracks could increase or decrease in width with seasonal variability among other factors. In any case, analysis of distress was performed under the assumption that all distress surveys were accurate at the time of observance.

Figure 14, Figure 15, and Figure 17 indicate that there is a strong correlation between 2015 PSR and $\mathrm{PCl}$. The comparison of trend lines and data points in Figure 16 verifies that the 2015 PSR method produces a distress based index that has a common scale for asphalt and concrete pavements. The linear relationship identified in Figure 17 can be used to adjust the thresholds for qualitative descriptors that are shown on Figure 7 as shown in Table 38 and Figure 53.

\begin{tabular}{|l|c|c|}
\hline Qualitative Descriptor & PCI & PSR \\
\hline Good & $85-100$ & $92-100$ \\
\hline Satisfactory & $70-85$ & $80-92$ \\
\hline Fair & $55-70$ & $68-80$ \\
\hline Poor & $40-55$ & $56-68$ \\
\hline Very Poor & $25-40$ & $44-56$ \\
\hline Serious & $10-25$ & $34-44$ \\
\hline Failed & $0-10$ & $0-34$ \\
\hline
\end{tabular}

Table 38: Comparison of PCI and 2015 PSR Ranges for Qualitative Descriptors 


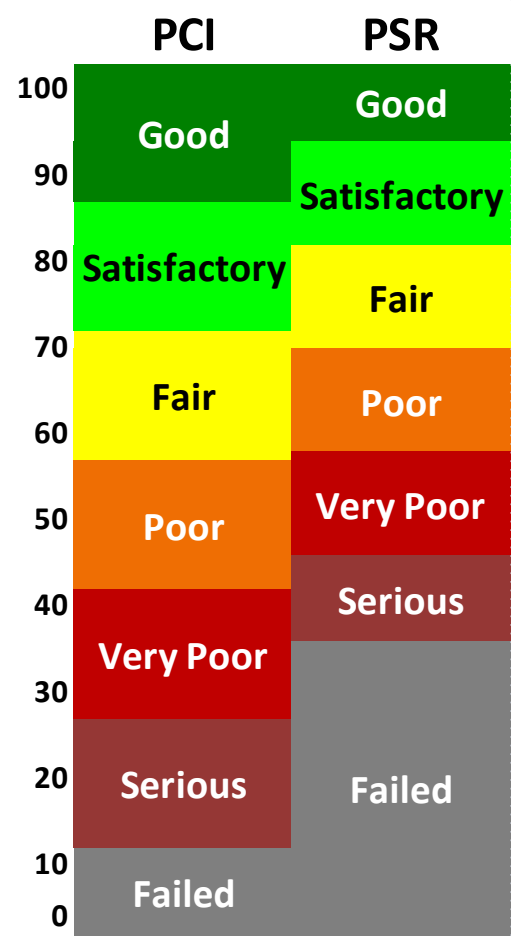

Figure 53: Graphical Comparison of PCl and 2015 PSR Ranges for Qualitative Descriptors

After the implementation of the proposed method for calculating PSR, a comparison of pavement types within US 35 was finally made. PSR was the only method of comparison used. Figure 54 shows the average asphalt and concrete PSR calculated for all test sections within US 35 . This includes fast and slow lanes in northbound and southbound directions. The data shows that the condition of the asphalt and concrete was reasonably similar until the fifth survey where asphalt displayed a noticeably larger decrease in PSR than concrete. In the fifth survey, the average asphalt PSR is approximately 91 which is below the proposed Good/Satisfactory threshold discussed in the previous section. This threshold and the range for "Good" and "Satisfactory" are included in Figure 54 through Figure 58. 


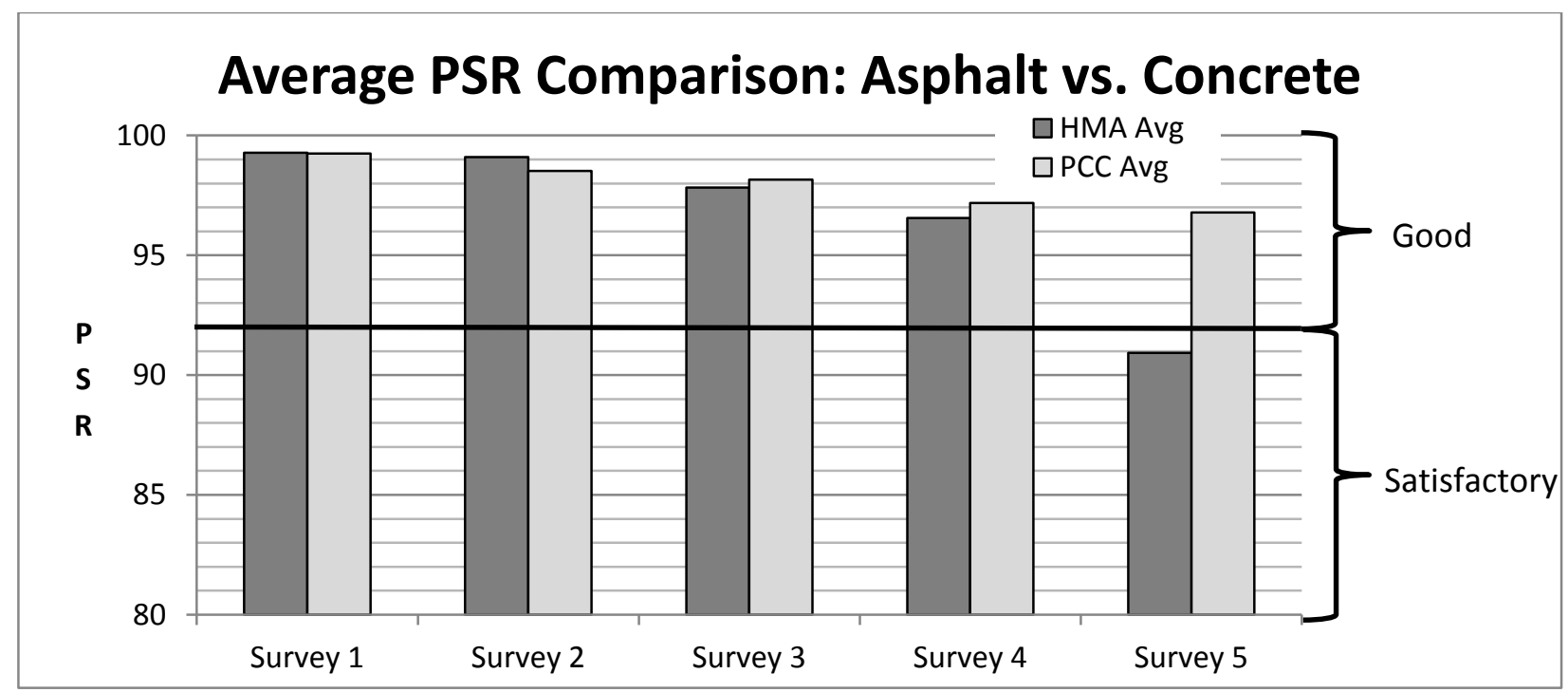

Figure 54: Average PSR Comparison: Asphalt vs. Concrete

Figure 55 and Figure 56 show fast lane and slow lane average PSR within the respective pavement type separately. For both pavement surface types, the slow lane was found to have a lower average PSR than the adjacent fast lane after each survey. However, there were instances of individual one-tenth mile test section fast lane average PSR values were found to be lower than the corresponding slow lane average PSR. The fact that slow lane PSR is on average consistently lower is to be expected due to the tendency of heavier and slower traffic in the lane. Interestingly, the difference between concrete fast and slow lane average PSR is much less drastic through all five surveys than in asphalt. The change in asphalt fast lane average PSR is unnoticeable through four surveys and displays a significant decrease at survey five.

Figure 57 and Figure 58 show asphalt and concrete average PSR as observed within the fast lane and slow lane separately. Interestingly, the fast lane asphalt average PSR is consistently higher than that of concrete for the first four surveys but falls below the fast lane average concrete PSR at survey five. Within both fast and slow lanes, concrete average PSR maintains relatively consistent decreases where asphalt average PSR drops most significantly (by numerical amount and percent change) between survey four and five. In Figure 54, we noted that the asphalt average PSR fell in the "Satisfactory" range. However, here, we notice that on average the fast lane asphalt PSR is within the "Good" range and the average slow lane average PSR is within the "Satisfactory" range. 


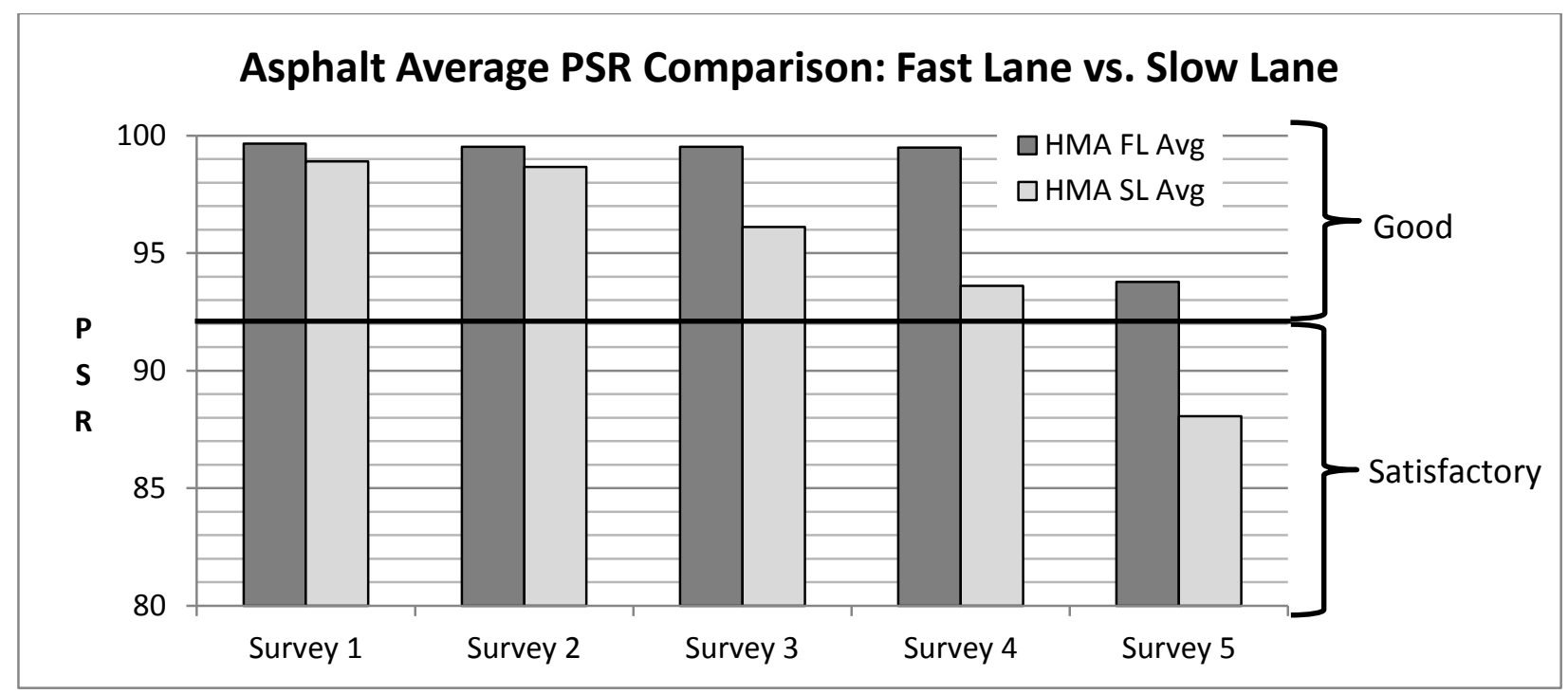

Figure 55: Asphalt Average PSR Comparison: Fast Lane vs. Slow Lane

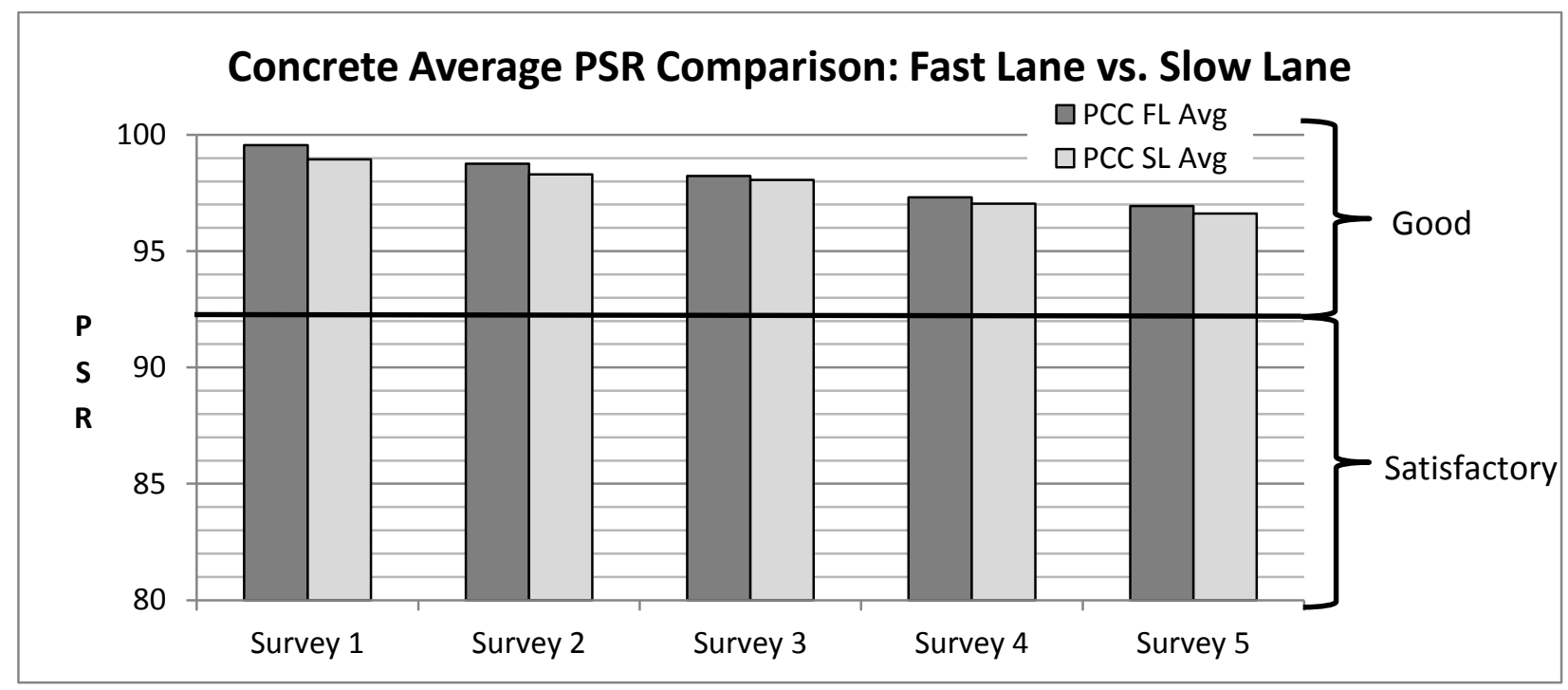

Figure 56: Concrete Average PSR Comparison: Fast Lane vs. Slow Lane 


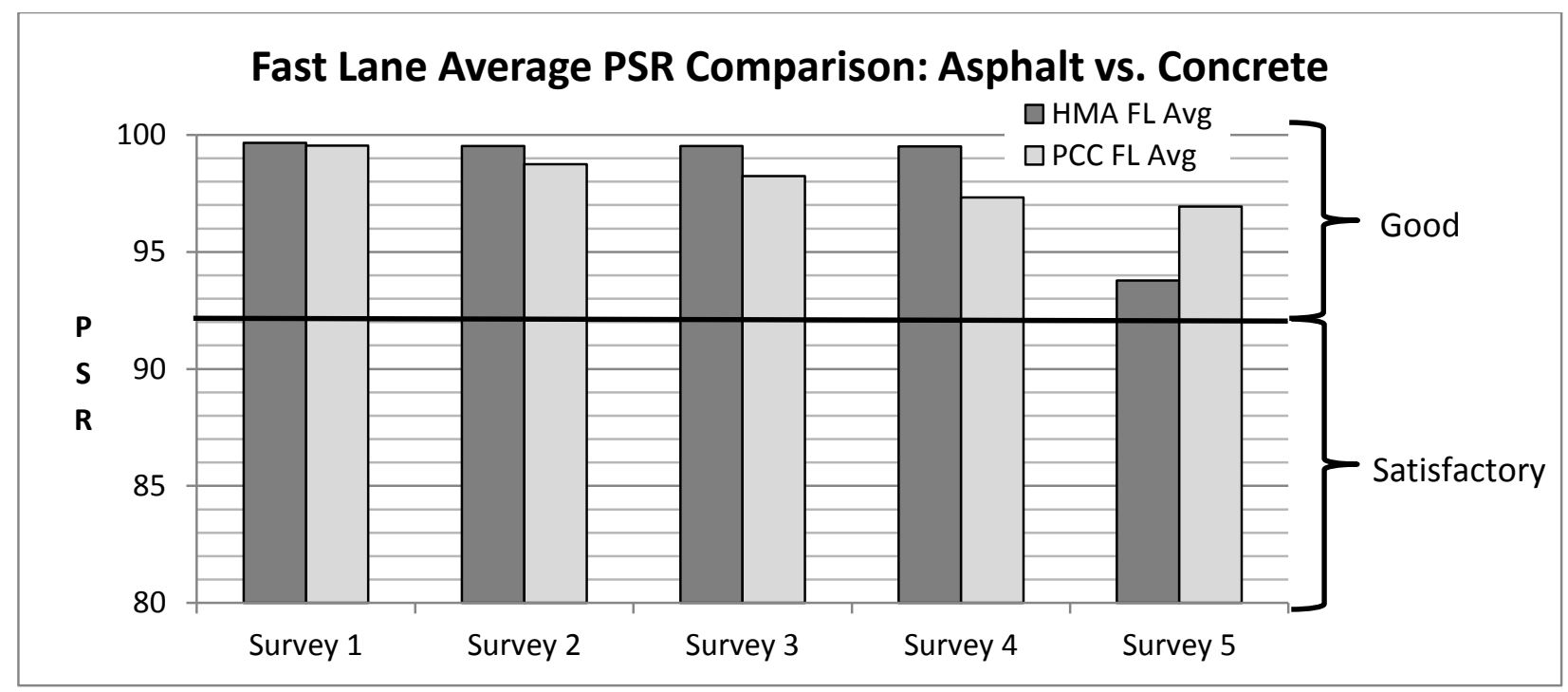

Figure 57: Fast Lane Average PSR Comparison: Asphalt vs. Concrete

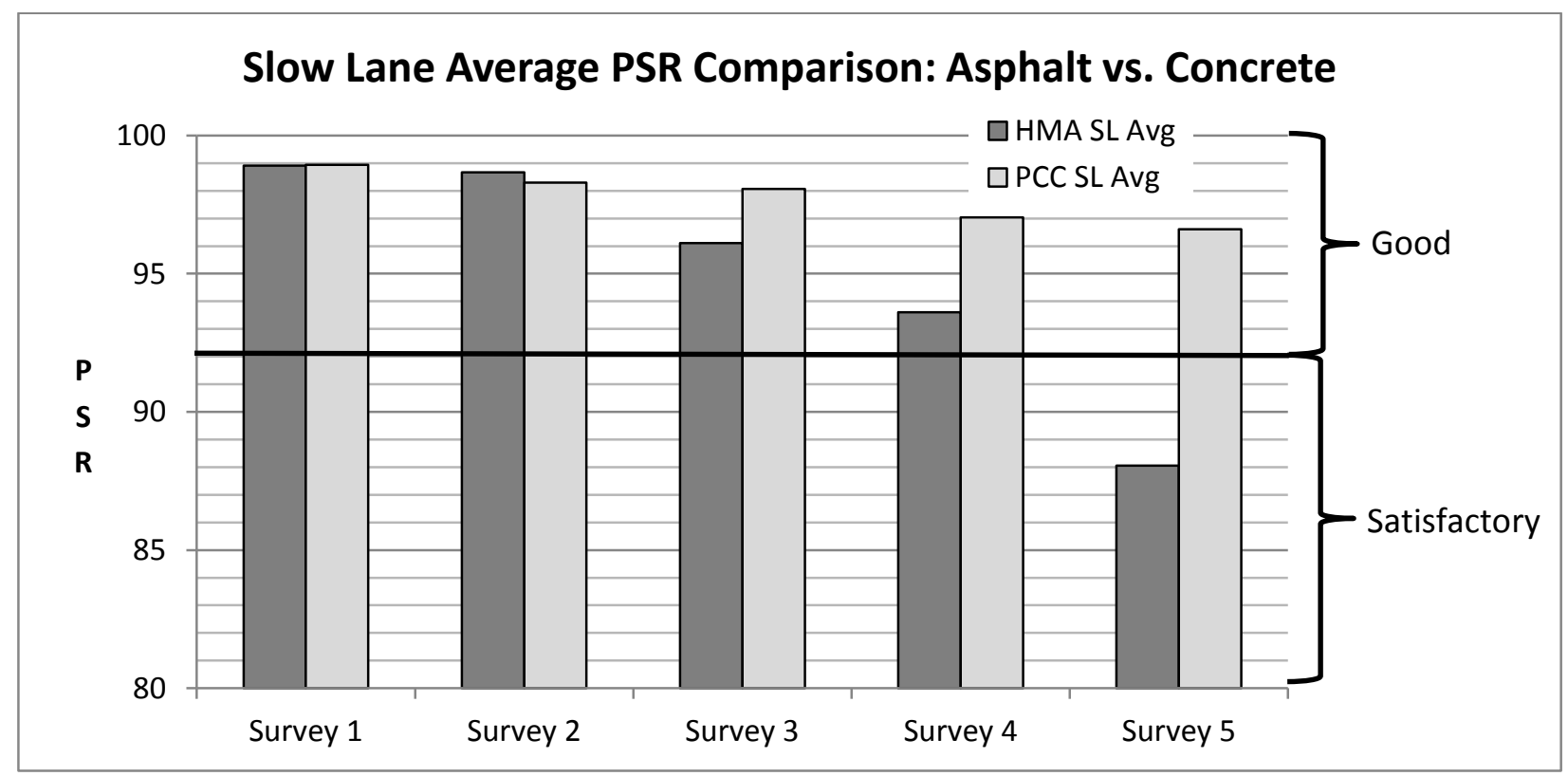

Figure 58: Slow Lane Average PSR Comparison: Asphalt vs. Concrete

\subsubsection{Comparison of ARAN and PSR Survey Surface Distress Data}

The comparison of the PMS data to data surveyed manually was inconclusive. Because PMS data were generally used for network level analysis, it may have been too ambitious to apply it in this manner. The distress data provided by the PMS is obviously more simplified than the data collected with the intent of calculating PSR. The majority of sections are reported to have no distresses besides 
roughness and minor rutting. In the case of $\mathrm{CSI}, \mathrm{ECl}$, and to some extent $\mathrm{SCl}$, both data sets agree that there are no distresses within the section. In many instances where distress is observed in manual surveys, there are still none observed within the PMS data. Overall, there may not be enough distress on the road to really have a chance of making any comparison.

\subsubsection{FWD Data Analysis}

A large majority of data from the concrete section of this project was not useful because the importance of drop location on a slab was not fully understood. Although the primary use of an FWD on Jointed Plain Concrete Pavement, JPCP, is Joint Load Transfer, JLT; structural properties of pavement layers can be determined if proper drop locations are used. For the purpose of this project, it would have been necessary to perform all drops in the centers of each slab.

Within the relevant tests in the asphalt section, some evidence is available that FWD testing could provide information on pavement structural properties. The sets of $\mathrm{SN}_{\text {eff }}$ values generated with $M_{R}$ were relatively consistent. The sets of $S N_{\text {eff }}$ values generated with $M_{R^{*}}$ were also relatively consistent, however, the two sets differed significantly from each other. For this reason, it is impossible to call either set inaccurate. The set generated with $M_{R}$ is more reasonable when considering the initial SN computed using the WVDOH Design Directive. Further investigation would be required to determine whether these computed $\mathrm{M}_{\mathrm{R}}$ values are comparable to those which are generated through laboratory tests.

\subsection{Recommendations}

\subsubsection{Pavement Surface Rating}

If in the future, a more traditional and consistent range for PSR pavement condition qualitative descriptors is desired, a simple adjustment to the PSR equation could be made.

The consistency and accuracy of the distress observations made by surveyors across time should be evaluated. This will allow evaluation of the variance in PSR as a function of the quality of the surveys. The variability in PSR results should be considered when determining changes in PSR are due to changes in pavement distress or are the result of variance in the distress survey.

The procedure outlined within the "Final PSR Calculation Procedure" section of Chapter 5 should be adopted by the WVDOH and used within the newest version of the "Section 490: Nine Year Pavement 
Performance Criteria." This includes the addition of the "WVDOH Distress Identification Manual (for PSR calculation)" within Appendix A.

\subsubsection{FWD Data and Analysis}

In order to place more emphasis on FWD deflection data and the information provided by the analysis of such data, there should be more practice and organization in collecting data. The reasons for the challenges with the use of the backcalculation programs should be evaluated. 


\section{References}

AASHTO 1993. AASHTO Guide for Design of Pavement Structures 1993. Washington, DC: American Association of State Highway and Transportation Officials, 1993.

Alavi, S., J.F. LeCates, and M.P. Tavares. Falling Weight Deflectometer Usage. Rep. no. NCHRP Synthesis 381. Washington, DC: TRB, 2008.

AINouri, I. Effect of Temperature on the Elastic Response of Asphalt Treated Base Material. Rep. no. R816. Lansing, MI, 1972.

ASTM D6433-11. Standard Practice for Roads and Parking Lots Pavement Condition Index Surveys. Vol. ASTM Standard D6433-11. West Conshohocken, PA, 2014.

Clark, T. Conversation about Application of FWD Data. Telephone interview. Nov. 2014.

Cornell University. Digital image. Cornell Local Roads Program. Cornell University, 2005

Fugro Roadware Inc. Fugro Roadware Inc. Mississauga, ON, CA: Fugro Roadware, 2012.

Gucunski, N., N. Vitillo, and S. Zaghloul. Development of FWD Procedures Manual. Rep. no. FHWA-NJ2009-005. Piscataway, NJ: NJDOT, 2009.

Killingsworh, B., and H. Von Quintus, Backcalculation of Layer Moduli of LTPP General Pavement Study (GPS) Sites. Rep. no. FHWA-RD-97-086. McLean, VA, 1997.

Jackson, N. Development of Revised Pavement Condition Indices for Portland Cement Concrete Pavement for the WSDOT Pavement Management System. Rep. no. WA-RD 682.3. Seattle, WA, 2008.

Lukanen, E.O., R. Stubstab, and R. Briggs. Temperature Predictions and Adjustment Factors for Asphalt Pavements. Rep. no. FHWA-RD-98-085. Mclean, VA: Federal Highway Administration, 2000.

Lytton, R. L., and F. P. Germann. "General Procedure for Backcalculating Layer Moduli." Ed. A. J. Bush and G. Y. Baladi. Nondestructive Testing of Pavements and Backcalculation of Moduli. By J. Uzan. Philadelphia, PA: American Society for Testing and Materials, 1989.

MnDOT, Office of Materials and Road Research Pavement Management Unit. Mn/DOT Distress Identification Manual. Maplewood, MN, 2003.

Norouzi, M. Performance Evaluation of Flexible Pavements in Alberta Using Falling Weight Deflectometer Data. Thesis. University of Alberta, 2013. Ottowa, Canada: Library and Archives Canada, 2013.

Pologruto, M. Procedure for Use of Falling Weight Deflectometer to Determine AASHTO Layer Coefficients. Rep. Vol. 1764. Washington, DC: Transportation Research Board, 2007. 
PCS/Law Engineering. Layer Moduli Backcalculation procedure: Software Selection. Rep. no. SHRP-P-651. Washington, DC: Transportation Research Board, 1993.

Sami, N. Composite Condition Index for West Virginia DOT: Final CCI Model. IMS Infrastructure Management Services, 1997.

Schmalzer, P. N. Long-Term Pavement Performance Program Manual for Falling Weight Deflectometer Measurements. Rep. no. FHWA-HRT-06-132. Vol. Version 4.1. McLean, VA: FHWA, 2006.

Shahin, M. Y. Pavement Management for Airports, Roads, and Parking Lots. 2nd ed. Boston, MA: Springer, 2005.

Section 490 Nine Year Pavement Performance Criteria, West Virginia Department of Transportation Division of Highways Special Provision. April 19, 2012.

Shoukry, S., and G. William. Performance Evaluation of Backcalculation Algorithms Through ThreeDimensional Finite-Element Modeling of Pavement Structures. Rep. no. 99-1277. Washington, DC: Transportation Research Board, 1999.

William, G., and S. Shoukry. Mechanistic Evaluation of the Depth to Bedrock and Its Effect on the Response of 3D FE Pavement Models. Http://ascelibrary.org/doi/abs/10.1061/40509(286)5. 1999.

WVDOH. Design Directives. Charleston WV: WVDOT, 2006. 


\section{Appendix A. WVDOH Distress Identification Manual (for PSR calculation)}

\section{A.1 Asphalt Surfaces}

These distress definitions, severity levels, and calculations are specific to asphalt surfaced roads manually surveyed for the calculation of PSR on $1 / 10^{\text {th }}$ mile long test sections as part of WVDOH Special Provision Section 490: Nine Year Pavement Performance Criteria. This may also include composite pavements (concrete pavements with asphalt overlays).

\section{A.1.1 Transverse Cracks - \#1}

\section{Definition}

Transverse cracks are any cracks within the travel lane predominantly perpendicular to the pavement centerline. This may include reflection cracking in composite pavements. In the case of full width patching that produces transverse construction joints, evaluate distresses in these joints as transverse cracks using the severity levels described in Longitudinal Joint Deterioration.

\section{Severity Level Identification}

Rate entire crack at highest level present for $10 \%$ or more of total crack length.

\section{Low}

- unsealed cracks $0-1 / 4$ inch wide, less than $1 / 4$ inch faulting, no blowups, no adjacent random cracking, and no loss of material

- sealed cracks with sealant material in good condition, no faulting, no blowups, no adjacent random cracking, and no loss of material

\section{Medium}

- any crack $1 / 4-3 / 4$ inch wide, less than $1 / 4$ inch faulting, no blowups, no adjacent random cracking, and no loss of material

- any crack less than $3 / 4$ inch wide with adjacent low severity cracking, less than $1 / 4$ inch faulting, no blowups, and no loss of material

\section{High}

- any crack greater than $3 / 4$ inch wide

- any crack with adjacent medium or high severity random cracking

- any crack with greater than $1 / 4$ inch faulting

- any crack with a noticeable blowup

- any crack with noticeable loss of material

\section{Extent Calculation}

Percentage is calculated by multiplying amount of cracks at each distress level by 2.86 . The sum of all three severities cannot exceed $100 \%$. If this happens, disregard cracks of the lowest severity present until the sum of percentages equals $100 \%$. 


\section{A.1.2 Edge Cracking - \#2}

\section{Definition}

Edge cracking refers to any crescent shaped or predominantly longitudinal cracks in the travel lane (including the paint stripe) that are within 2 feet of the pavements edge. This can occur only where there is an unpaved shoulder. Edge cracking may include longitudinal reflection cracking in composite pavements.

\section{Severity Level Identification}

Rate entire crack at highest level present for $10 \%$ or more of total crack length.

\section{Low}

- unsealed cracks $0-1 / 4$ inch wide and no loss of material

- sealed cracks with sealant material in good condition

\section{Medium}

- any crack $1 / 4-3 / 4$ inch wide and no loss of material

\section{High}

- any crack greater than $3 / 4$ inch wide

- any crack with noticeable loss of material or loose pieces of pavement

\section{Extent Calculation}

For each severity, the total length of edge cracking measured in linear feet will be divided by 528 and then multiplied by 100 to determine the percent of test section affected. In the unlikely event of a one lane road with unpaved shoulders on both sides of the travel lane surveyed, the calculation will remain the same however now there will be the potential for a maximum of $200 \%$

\section{A.1.3 Alligator Cracking - \#3}

\section{Definition}

Alligator cracking occurs only in areas subjected to repeated traffic loadings (wheelpaths). The exact location of the wheelpath is based on the inspector's judgment but will usually occur approximately 1.5 feet from paint stripes and extend about 2.5 feet from that point. Alligator cracks appear as interconnected cracks in early stages and may develop into many sided, sharp angled pieces, usually less than 1 foot in any dimension, characteristically with a chicken wire/alligator pattern. Meandering longitudinal cracking with adjacent random cracking can be considered alligator cracking if it is within the wheelpath. In this case it cannot also be counted as longitudinal cracking.

\section{Severity Level Identification}

Severity of alligator cracking can change between each linear foot of pavement. Rate cracks at highest level present for $10 \%$ or more within each linear foot where appropriate. If severity changes frequently, inspector's judgment should be applied. 


\section{Low}

- an area with only a few connecting cracks and no noticeable loss of material

\section{Medium}

- an area of interconnecting cracks that forms a complete pattern but no considerable loss of material

\section{High}

- an area of interconnecting cracks with noticeable loss of material or loose pieces

\section{Extent Calculation}

For each severity, the total length of alligator cracking measured in linear feet will be divided by 528 and then multiplied by 100 to determine the percent of test section affected. If alligator cracking is present in both wheelpaths, consider only the higher severity within each linear foot. For example, if a ten foot section of pavement exhibits low severity alligator cracking in the left wheelpath and high severity alligator cracking in the right wheelpath, only 10 feet of high severity alligator cracking should be recorded. If that same section exhibit low severity alligator cracking in both wheelpaths, only 10 feet of low severity alligator cracking should be recorded.

\section{A.1.4 Block Cracking - \#4}

\section{Definition}

Block cracking is a pattern of cracks that divides the pavement into approximately rectangular pieces greater than 1 foot and less than 10 feet in any dimension. This can occur anywhere within the lane. If a series of cracks is identified as block cracking, it should not also be counted as any other distress.

\section{Severity Level Identification}

Severity of block cracking can change between each linear foot of pavement. Rate cracks at highest level present for $10 \%$ or more of each section within the block cracking pattern. If severity changes frequently, inspector's judgment should be applied.

\section{Low}

- unsealed cracks $0-1 / 4$ inch wide, no adjacent random cracking, and no loss of material

- sealed cracks with sealant material in good condition

\section{Medium}

- any crack $1 / 4-3 / 4$ inch wide, no adjacent random cracking, and no loss of material

- any crack less than $3 / 4$ inch wide with adjacent low severity cracking, and no loss of material

\section{High}

- any crack greater than $3 / 4$ inch wide

- any crack with adjacent medium or high severity random cracking

- any crack with noticeable loss of material 


\section{Extent Calculation}

For each severity, the total length of block cracking measured in linear feet will be divided by 528 and then multiplied by 100 to determine the percent of test section affected.

\section{A.1.5 Longitudinal Cracking - \#5}

\section{Definition}

Longitudinal cracks are any cracks within the travel lane predominantly parallel with the pavement centerline. This may include reflection cracking in composite pavements. If a crack has been identified as longitudinal cracking, it should not be counted as any other distress.

\section{Severity Level Identification}

Severity of longitudinal cracking can change between each linear foot of pavement. Rate cracks at highest level present for $10 \%$ or more within each linear foot where appropriate. If severity changes frequently, inspector's judgment should be applied.

\section{Low}

- unsealed cracks $0-1 / 4$ inch wide, no adjacent random cracking, and no loss of material

- sealed cracks with sealant material in good condition, no adjacent random cracking, and no loss of material

\section{Medium}

- any crack $1 / 4-3 / 4$ inch wide, no adjacent random cracking, and no loss of material

- any crack less than $3 / 4$ inch wide with adjacent low severity cracking, and no loss of material

\section{High}

- any crack greater than $3 / 4$ inch wide

- any crack with adjacent medium or high severity random cracking

- any crack with noticeable loss of material

\section{Extent Calculation}

For each severity, the total length of longitudinal cracking measured in linear feet will be divided by 528 and then multiplied by 100 to determine the percent of test section affected. If multiple longitudinal cracks are present in any linear foot of the lane, record only the highest severity within each linear foot.

\section{A.1.6 Longitudinal Joint Deterioration - \#6}

\section{Definition}

Longitudinal joint deterioration refers to any distresses affecting the deterioration of longitudinal construction joints found entirely within travel lanes. Any distress occurring exclusively within six inches of a longitudinal joint should be counted only as joint deterioration. Any longitudinal joint located within (or 6 inches from) the paint line separating a lane from a shoulder should be evaluated with the lane edge cracking distress. Otherwise count 
deterioration in whichever lane the joint is located. If a longitudinal joint is found within the divider line between travel lanes, consider that joint a part of the slower (right) lane.

\section{Severity Level Identification}

Severity of longitudinal joint deterioration can change between each linear foot of pavement. Rate cracks at highest level present for $10 \%$ or more within each linear foot where appropriate. If severity changes frequently, inspector's judgment should be applied.

\section{Low}

- unsealed crack $0-1 / 4$ inch wide propagating from the joint into the pavement, no adjacent random cracking

- minor loss of material on either side of a construction joint and no adjacent random cracking within 6 inches of the joint

- any other low severity distress located within 6 inches of a joint

\section{Medium}

- a crack $1 / 4-3 / 4$ inch wide propagating from the joint into the pavement, no adjacent random cracking

- a crack less than $3 / 4$ inch wide propagating from the joint into the pavement, adjacent random cracking less than $1 / 4$ inch wide

- minor loss of material on either side of a construction joint and adjacent random cracking less than $1 / 4$ inch wide

- any other medium severity distress located within 6 inches of a joint

\section{High}

- a crack greater than $3 / 4$ inch wide propagating from the joint into the pavement

- a crack propagating from the joint into the pavement with adjacent random cracking greater than $1 / 4$ inch wide

- major loss of material on either side of a construction joint

- minor loss of material on either side of a construction joint and adjacent random cracking greater than $1 / 4$ inch wide

\section{Extent Calculation}

For each severity, the total length of longitudinal joint deterioration measured in linear feet will be divided by 528 and then multiplied by 100 to determine the percent of test section affected. In the unlikely event that multiple longitudinal joints are found within a single lane, percent affected could exceed $100 \%$.

\section{A.1.7 Lane Edge Cracking/Deterioration - \# 7}

\section{Definition}

Lane edge cracking/deterioration refers longitudinal joint deterioration specifically between a travel lane and a paved shoulder. A paved shoulder here is defined by at least two feet of pavement past the paint stripe. Lane edge cracking/deteriorations includes any distress which occurs or stretches into the paint stripe or first 6 inches of lane. All definitions of longitudinal 
joint deterioration apply no to this location. Additionally lane edge cracking will include cracking in the paint stripe which propagates into the pavement and is caused by the paint itself.

\section{Severity Level Identification}

Severity of lane edge cracking/deterioration can change between each linear foot of pavement. Rate cracks or deterioration at highest level present for $10 \%$ or more within each linear foot where appropriate. If severity changes frequently, inspector's judgment should be applied.

\section{Low}

- unsealed crack $0-1 / 4$ inch wide propagating from the paint into the pavement, no adjacent random cracking

- unsealed crack $0-1 / 4$ inch wide propagating from the joint into the pavement, no adjacent random cracking

- minor loss of material on either side of a construction joint and no adjacent random cracking within 6 inches of the joint

- any other low severity distress located within 6 inches of a joint

\section{Medium}

- a crack $1 / 4-3 / 4$ inch wide propagating from the paint into the pavement, no adjacent random cracking

- a crack less than $3 / 4$ inch wide propagating from the paint into the pavement, adjacent random cracking less than $1 / 4$ inch wide

- a crack $1 / 4-3 / 4$ inch wide propagating from the joint into the pavement, no adjacent random cracking

- a crack less than $3 / 4$ inch wide propagating from the joint into the pavement, adjacent random cracking less than $1 / 4$ inch wide

- minor loss of material on either side of a construction joint and adjacent random cracking less than $1 / 4$ inch wide

- $\quad$ any other medium severity distress located within 6 inches of a joint

\section{High}

- a crack greater than $3 / 4$ inch wide propagating from the paint into the pavement

- a crack propagating from the paint into the pavement with adjacent random cracking greater than $1 / 4$ inch wide

- a crack greater than $3 / 4$ inch wide propagating from the joint into the pavement

- a crack propagating from the joint into the pavement with adjacent random cracking greater than $1 / 4$ inch wide

- major loss of material on either side of a construction joint

- minor loss of material on either side of a construction joint and adjacent random cracking greater than $1 / 4$ inch wide

\section{Extent Calculation}

For each severity, the total length of lane edge cracking/deterioration measured in linear feet will be divided by 528 and then multiplied by 100 to determine the percent of test section affected. 


\section{A.1.8 Raveling/Weathering - \#8}

\section{Definition}

Raveling and weathering refer to the wearing of pavement surface characterized by the dislodging of aggregate particles and loss of asphalt binder. It may range from loss of fines and binder between coarse aggregate to very rough and pitted surface with obvious loose aggregate.

\section{Severity Level Identification}

No severity levels

\section{Extent Calculation}

Raveling/weathering is recorded in linear feet. If any of the pavement within a linear foot of the lane appears to be raveled/weathered then it should be recorded. The total length of raveling/weathering measured in linear feet will be divided by 528 and then multiplied by 100 to determine the percent of test section affected.

\section{A.1.9 Patching - \#9}

\section{Definition}

Patching refers to any portion of pavement surface that has been replaced or added to after the original construction. This should include full depth repairs. All other distresses found within the patch should still be recorded.

\section{Severity Level Identification}

No severity levels

\section{Extent Calculation}

Patching of any type, width, depth, etc. within the lane is recorded in linear feet. The total length of patching measured in linear feet will be divided by 528 and then multiplied by 100 to determine the percent of test section affected.

\section{A.1.10 Rutting - \#10}

\section{Definition}

Rutting refers to pavement surface depression due to repeated loading, within the wheelpath, and occurring in the longitudinal direction.

\section{Severity Level Identification}

Rutting severity is based on the average rut depth of 5 measurements in each wheelpath. These measurements should be taken approximately at the $64^{\text {th }}, 164^{\text {th }}, 264^{\text {th }}, 364^{\text {th }}$, and $464^{\text {th }}$ foot of the test section. These rut measurements will be taken using a 5 foot long straight edge, placed perpendicular to the pavement centerline. Rut depth is measured at the point of greatest distance from the bottom of the straightedge to the pavement surface. Measurements must be 
accurate to the closest $1 / 16^{\text {th }}$ inch. If the average rut depth for the five measurements in either wheelpath rut depth qualify for a severity, then that entire test section is recorded at that severity. Also if both wheelpaths qualify, the higher severity should be recorded.

\section{Low}

- average rut depth $1 / 4-1 / 2$ inch

\section{High}

- average rut depth greater than $1 / 2$ inch

\section{Extent Calculation}

If the average rut depth qualifies for a severity, then $100 \%$ of that section is recorded at that severity.

\section{A.2 Concrete Surfaces}

These distress definitions, severity levels, and calculations are specific to all concrete surfaced roads manually surveyed for the calculation of PSR on $1 / 10^{\text {th }}$ mile long test sections as part of WVDOT Division of Highways Special Provision Section 490: Nine Year Pavement Performance Criteria. This may include jointed reinforced concrete pavements, jointed plain concrete pavements, and continuously reinforced concrete pavements. Surveys on pavements with concrete surfaces require an input for number of joints within the $1 / 10^{\text {th }}$ mile segment as some extent calculations are dependent on this.

\section{A.2.1 Transverse Cracks - \#11}

\section{Definition}

Transverse cracks are cracks predominantly perpendicular to the pavement centerline and propagate through the width of the slab. This may count a linear crack that separates a corner of a slab. This is sometimes referred to as a corner break but should be recorded as a transverse crack if it is predominantly perpendicular to the pavement centerline.

\section{Severity Level Identification}

Rate entire crack at highest level present for $10 \%$ or more of total crack length.

\section{Low}

- unsealed cracks $0-1 / 8$ inch wide, no faulting, no spalling

- sealed cracks with sealant material in good condition, no faulting, no spalling

\section{Medium}

- $\quad$ any crack $1 / 8-1 / 4$ inch wide, no faulting, no spalling

- any crack less than $1 / 4$ inch wide with faulting up to $1 / 4$ inch

- any crack less than $1 / 4$ inch wide with spalling up to 3 inches wide

- any crack that would otherwise be considered Low Severity but is accompanied by additional Low Severity cracks that fragment the slab 


\section{High}

- any crack greater than $1 / 4$ inch wide

- any crack with greater than $1 / 4$ inch faulting

- any crack with greater than 3 inch wide spalling

- any crack that would otherwise be considered Medium Severity but is accompanied by additional Low or Medium Severity cracks that fragment the slab

\section{Extent Calculation}

Percentage is calculated by multiplying amount of cracks at each distress level by 2.86 . The sum of all three severities cannot exceed $100 \%$. If this happens, disregard cracks of the lowest severity present until the sum of percentages equals $100 \%$.

\section{A.2.2 Transverse Joint Spalls - \#12}

\section{Definition}

Transverse joint spalling refers to cracking, breaking, chipping, or fraying of slab edges at transverse joints. Spalling includes any cracking within 1 foot of the face of the joint. Spalling is counted on a per joint basis so it does not matter if the spalling occurs on the leave or approach slab. Spall width is based on the distance from the face of the joint to the end of displaced material on either side of the joint. If no material is displaced but cracking is still present, the spall width is zero but it is still considered spalled.

\section{Severity Level Identification}

Rate a joints spalling as the highest severity present for at least $10 \%$ of the joint length. Spalling is recorded on a per joint basis so the entire joint is considered spalled at that severity.

\section{Low}

- $\quad$ spall widths up to 2 inches

- joints with low severity cracking in crescent shaped patterns or parallel to the joint within 1 foot of the joint

\section{Medium}

- $\quad$ spall widths $2-4$ inches

\section{High}

- $\quad$ spall widths greater than 4 inches

\section{Extent Calculation}

Record the number of transverse joints with spalling at each severity level. Divide the amount of affected joints at each severity level by the total number of joints in the segment. Multiply by 100 to determine the percentage of segment affected. 


\section{A.2.3 Transverse Joint Faults - \#13}

\section{Definition}

Transverse joint faulting refers to the difference in elevation across a transverse joint. This can be an increase or decrease in elevation from the leave to the approach slab. It is recorded on a per joint basis.

\section{Severity Level Identification}

Rate a joints faulting as the highest severity present for at least $10 \%$ of the joint length. Faulting is recorded on a per joint basis so the entire joint is considered faulted at that severity.

\section{Low}

- measurable faulting to a maximum of $1 / 8$ inch

\section{Medium}

- faulting greater than $1 / 8^{\text {th }}$ inch but less than $1 / 2$ inch

\section{High}

- faulting $1 / 2$ inch or greater

\section{Extent Calculation}

Record the number of transverse joints with faulting at each severity level. Divide the amount of affected joints at each severity level by the total number of joints in the segment. Multiply by 100 to determine the percentage of segment affected.

\section{A.2.4 Blowups - \#14}

\section{Definition}

Blowups refer to localized upward movement of pavement surface at transverse joints, possible accompanied by shattering of concrete in the area on either side of the joint. Blowups can occur mid-slab at a transverse crack but these should will not be counted in this distress. If a joint is experiencing a blowup, there is no need to also record faulting or spalling at that joint

\section{Severity Level Identification}

No severity levels

\section{Extent Calculation}

Record the number of transverse joints with a blowup. Divide the amount of affected joints at by the total number of joints in the segment. Multiply by 100 to determine the percentage of segment affected. 


\section{A.2.5 Longitudinal Cracking - \#15}

\section{Definition}

Longitudinal cracks are cracks predominantly parallel to the pavement centerline and propagate through the length of the slab. This may count a linear crack that separates a corner of a slab. This is sometimes referred to as a corner break but should be recorded as a longitudinal crack if it is predominantly parallel to the pavement centerline.

\section{Severity Level Identification}

Rate the entire crack within a single slab at the highest level present for $10 \%$ or more of total crack length. Longitudinal cracks are recorded on a linear foot basis so if multiple longitudinal cracks are present on a single slab, record it as a single crack and increase the severity.

\section{Low}

- unsealed cracks $0-1 / 8$ inch wide, no faulting, no spalling

- sealed cracks with sealant material in good condition, no faulting, no spalling

\section{Medium}

- any crack $1 / 8-1 / 4$ inch wide, no faulting, no spalling

- any crack less than $1 / 4$ inch wide with faulting up to $1 / 4$ inch

- any crack less than $1 / 4$ inch wide with spalling up to 3 inches wide

- any crack that would otherwise be considered Low Severity but is accompanied by additional Low Severity cracks that fragment the slab

\section{High}

- any crack greater than $1 / 4$ inch wide

- any crack with greater than $1 / 4$ inch faulting

- any crack with greater than 3 inch wide spalling

- any crack that would otherwise be considered Medium Severity but is accompanied by additional Low or Medium Severity cracks that fragment the slab

\section{Extent Calculation}

For each severity, the total length of longitudinal cracking measured in linear feet will be divided by 528 and then multiplied by 100 to determine the percent of test section affected.

\section{A.2.6 Longitudinal Joint Spalling - \#16}

\section{Definition}

Longitudinal joint spalling refers to any cracking, breaking, chipping, or fraying of slab edges at longitudinal construction joints found entirely within travel lanes. Spalling includes any cracking within 1 foot of the face of the joint. Any longitudinal joint located within (or 1 foot from) the paint line separating a lane from a shoulder should be evaluated with the lane edge spalling distress. Otherwise count spalling in whichever lane the joint is located. If a longitudinal joint is found within the divider line between travel lanes, consider that joint a part of the slower (right) lane. Spalling is counted as a distress to the joint so it does not matter which side of the joint 
experiences spalling. Spall width is based on the distance from the face of the joint to the end of displaced material on either side of the joint. If no material is displaced but cracking is still present, the spall width is zero but it is still considered spalled.

\section{Severity Level Identification}

Severity of longitudinal joint spalling can change between each linear foot of pavement. Rate spalls at highest level present for $10 \%$ or more within each linear foot where appropriate. If severity changes frequently, inspector's judgment should be applied.

\section{Low}

- $\quad$ spall widths up to 2 inches

- joints with low severity cracking in crescent shaped patterns or parallel to the joint within 1 foot of the joint

\section{Medium}

- $\quad$ spall widths $2-4$ inches

\section{High}

- $\quad$ spall widths greater than 4 inches

\section{Extent Calculation}

For each severity, the total length of longitudinal joint spalling measured in linear feet will be divided by 528 and then multiplied by 100 to determine the percent of test section affected. If multiple longitudinal joints are found within a single lane, percent affected could exceed $100 \%$. This is relevant when slab widths are smaller than lane widths.

\section{A.2.7 Lane Edge Spalling - \#17}

\section{Definition}

Lane edge spalling refers longitudinal joint spalling specifically between a travel lane and a shoulder. This includes both paved and unpaved shoulders. Spall width is based on the distance from the face of the joint to the end of displaced material on either side of the joint. If no material is displaced but cracking is still present, the spall width is zero but it is still considered spalled. In the case of unpaved shoulders, where there is technically no joint, spalling still can be experienced on the edge of the slab.

\section{Severity Level Identification}

Severity of lane edge spalling can change between each linear foot of pavement. Rate spalls at highest level present for $10 \%$ or more within each linear foot where appropriate. If severity changes frequently, inspector's judgment should be applied.

\section{Low}

- spall widths up to 3 inches

- joints with low severity cracking in crescent shaped patterns or parallel to the joint within 1 foot of the joint 


\section{Medium}

- spall widths 3-6 inches

High

- $\quad$ spall widths greater than 6 inches

\section{Extent Calculation}

For each severity, the total length of lane edge spalling measured in linear feet will be divided by 528 and then multiplied by 100 to determine the percent of test section affected. In the unlikely event of a one lane road with shoulders (paved or unpaved) on either side, lane edge spalling could be present on both sides, therefore, percent affected could exceed $100 \%$.

\section{A.2.8 D Cracking - \#18}

\section{Definition}

D cracking, sometimes known as durability cracking, refers to closely spaced hairline cracking crescent shaped patterns that follow the edge of a slab near all joints. This is a very distinct distress that is usually caused by un-ideal properties of aggregate in concrete. It can increase in severity to entire slabs showing this patterned crack with significant loss of material across the surface. It is not to be confused with spalling which generally leads to loss of material specifically at the joint, or scaling which is usually a more random cracking pattern in the surface of the concrete.

\section{Severity Level Identification}

No severity levels

\section{Extent Calculation}

The total length of $D$ cracking measured in linear feet will be divided by 528 and then multiplied by 100 to determine the percent of test section affected.

\section{A.2.9 Scaling - \#19}

\section{Definition}

Scaling, also known as map cracking, refers to a series of interconnected cracks in the surface of concrete. This can range in severity from noticeable interconnected hairline cracks, to sections with significant loss of material across the surface. Generally, scaling will not result in displaced material deeper than $1 / 2$ inch from the original pavement surface.

\section{Severity Level Identification}

No severity levels

\section{Extent Calculation}

The total length of scaling measured in linear feet will be divided by 528 and then multiplied by 100 to determine the percent of test section affected. 


\section{A.2.10 Patching - \#20}

\section{Definition}

Patching refers to any portion of pavement surface that has been replaced or added to after the original construction. Full depth, full width repairs will not be considered patches. However, it should be noted that full depth repairs could change the amount of joints and slabs within test sections for future surveys. All other distresses found within the patch should still be recorded.

\section{Severity Level Identification}

No severity levels

\section{Extent Calculation}

Patching of any type, width, depth, etc. within the lane is recorded in linear feet. The total length of patching measured in linear feet will be divided by 528 and then multiplied by 100 to determine the percent of test section affected.

\section{A.2.11 Popouts - \#21}

\section{Definition}

Popouts are small pieces of pavement broken loose from the surface, normally ranging in diameter from $1-4$ inches and depth from $1 / 2-2$ inches.

\section{Severity Level Identification}

No severity levels

\section{Extent Calculation}

Sections of pavement with, on average, more than 3 popouts per square yard should be recorded as experiencing excessive popouts. This is not to say that one square yard with 3 popouts must be noted, but rather take note if significant sections of pavement or entire slabs appear to exceed this average popout density. This will require the inspector's judgment. Popouts are measured on a linear foot basis, so record the length of sections, or the length of slabs exhibiting excessive popouts. The total length in linear feet will be divided by 528 and then multiplied by 100 to determine the percent of test section affected. 


\section{Appendix B. Deduct Curve Comparison}

The comparison of deduct curves for each severity of every distress which led to the final weight factors within the respective PSR calculation model is included here. The final weight factor for each severity of every distress as well as the rationale for it is noted. Within each figure's legend, "ASTM Deduct Value" refers to the deduct values read directly from the curves within PCI's ASTM. "PSR Deduct Value" refers to the adaption of weight factors to deduct curve by applying the array of extents to the isolated distress and calculating TWD as in PSR's calculation model.

Deduct Curve Ratio (DCR) will be used exclusively here as a quick description of the theoretical difference between curves in PCl and PSR for asphalt surface distresses. It is defined as the ratio of PCI to PSR deduct value at any given extent percentage. This is necessitated by the difference in recording extent in either method. For instance, a 528 foot long longitudinal crack would be recorded as having an extent of $8.33 \%$ in PCl (for a 12 foot wide lane) and 100\% in PSR. In this case, a DCR of approximately 12:1 would be justifiable. Additionally this difference is the reason many PCI Deduct curves do not exist beyond $20-30 \%$. By definition, some distresses within $\mathrm{PCl}$ are impossible to exist on $100 \%$ of the pavement surface. PSR uses an extent calculation system which could be more closely defined as the percent of area exhibiting distress symptoms of all the area that could be affected by that distress. After comparing definitions of severity levels this was usually the first modification made to the PSR deduct curves. It is impossible to perfectly match curves based on DCR alone because often the curves will have a different curvature so this was used as a tool to establish an approximate theoretical match. This was not a factor within concrete surface distresses as those are calculated for $\mathrm{PCl}$ on a joint or slab basis.

After this, a reduction of PSR curves was usually made as PCl includes a built in correction within its procedure where PSR does not. This again was an approximation because the $\mathrm{PCl}$ deduct correction is dependent on the total amount of distress in a section. From experience, in normal situations where 2 or 3 different distresses are recorded, this correction may reduce the deduct value to about $70-80 \%$ of the original. As distresses mount, the reduction factor is increased to the point where the final deduct value could be $40 \%$ of the original value if 7 or 8 different distresses are recorded. For most distresses, the PSR deduct curve was reduced to about $60 \%$ of the curve set by DCR. Without a dynamic correction factor, PSR when compared to PCl could theoretically be underestimating pavement distress in situations where few are present and overestimate when many are present. For the purpose of maintaining simplicity this was deemed acceptable as a starting point for comparisons. 
In a few situations slight changes in distress definitions and naming necessitated the modifications of curves where intuition and experience would be required to establish reasoning. Also, in a few select cases senior engineer judgment and input was factored. A few requests were made based on the preferences of the DOH office to slightly increase or reduce weight factors to address their own concerns.

PSR final weight factors are listed from Low to High in the first bullet of each distress for as many weight factors defined for each distress. This is followed by brief descriptions to the reasoning for any or all modifications. Then last, the deduct comparison curves used are included.

\section{B.1 Deduct Comparison for Asphalt Surfaces}

\section{Transverse Cracks:}

- $\quad 0.020,0.110,0.200$

- Severity definitions are similar with the exception of crack width thresholds which are more lenient within the $\mathrm{PCl}$ definition, especially at High Severity.

- Initial DCR of approximately 3:1

- Additional decrease to Low Severity because perceived distress is minimal at this level.

- Inclusion of Faulting and especially the potential for Blowups within composite pavements resulted in the increase of Medium and High Severity curves.

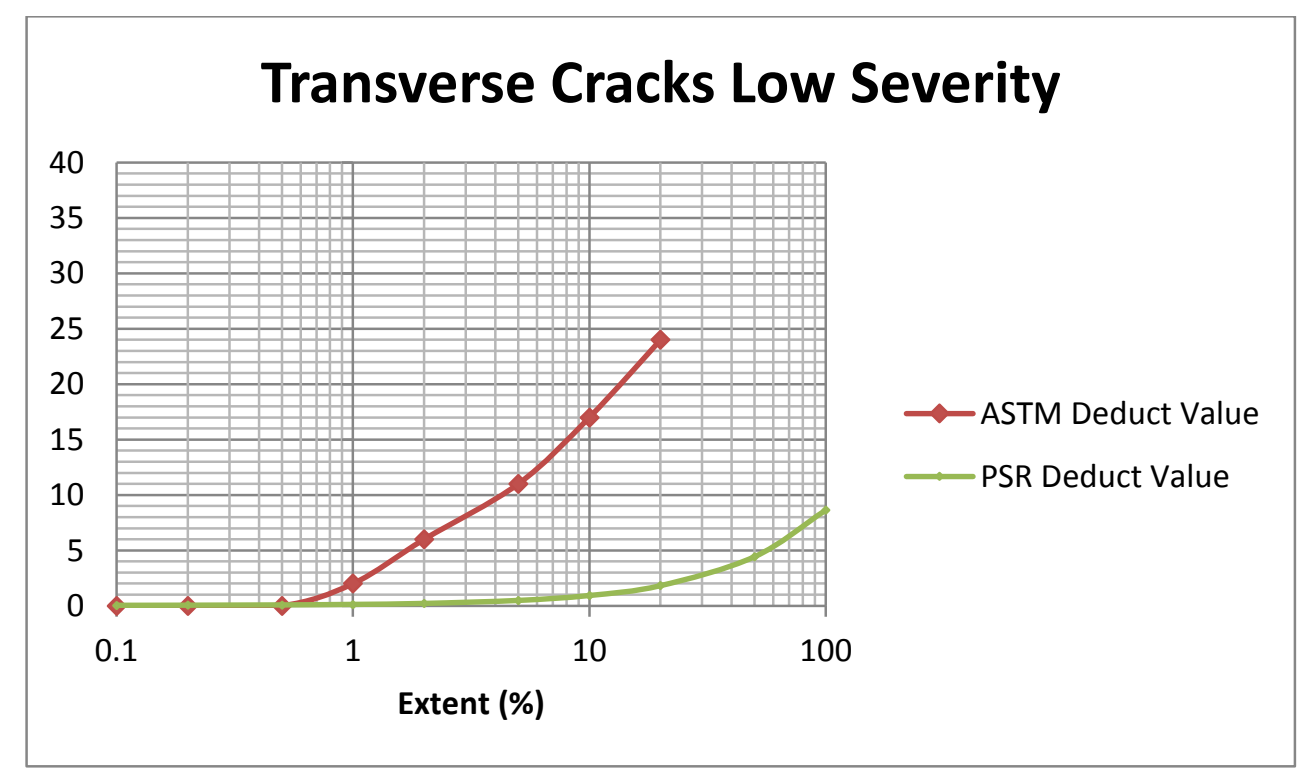

Figure 59:Transverse Cracks Low Severity Deduct Curve Comparison 


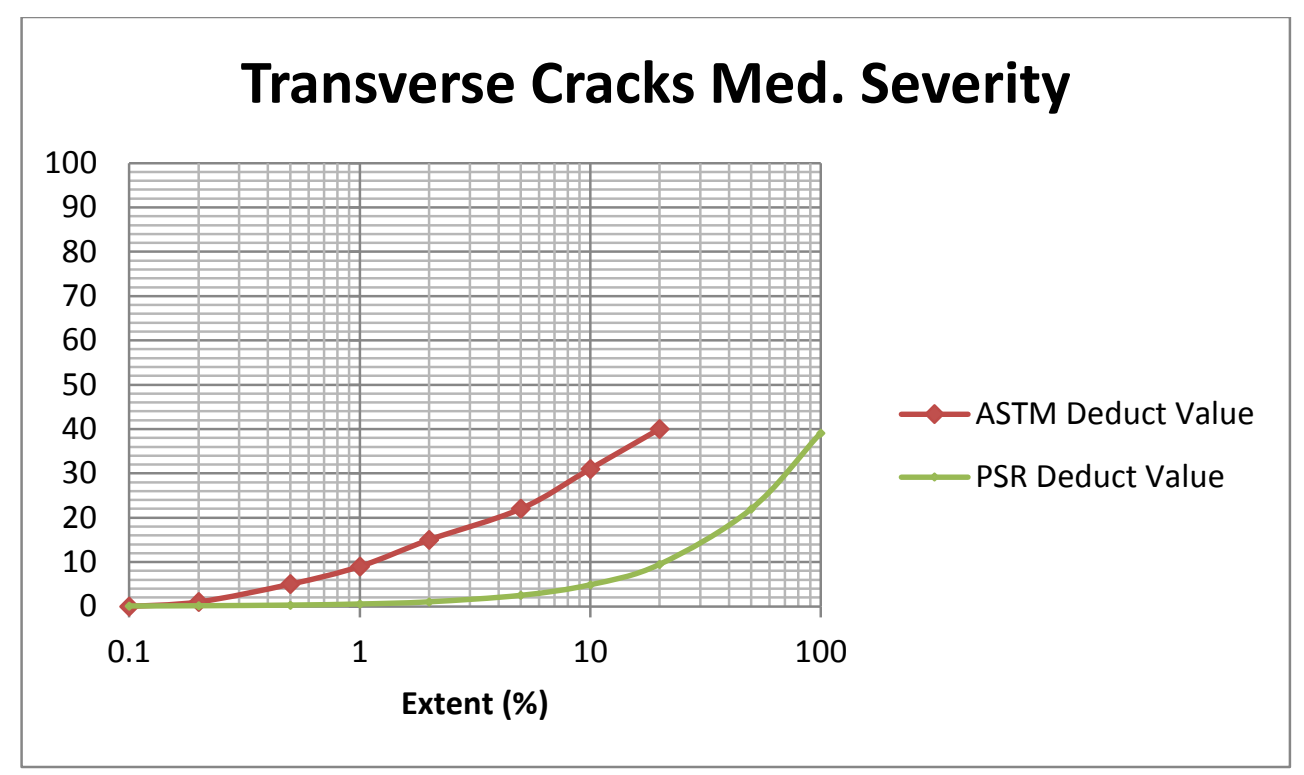

Figure 60: Transverse Cracks Medium Severity Deduct Curve Comparison

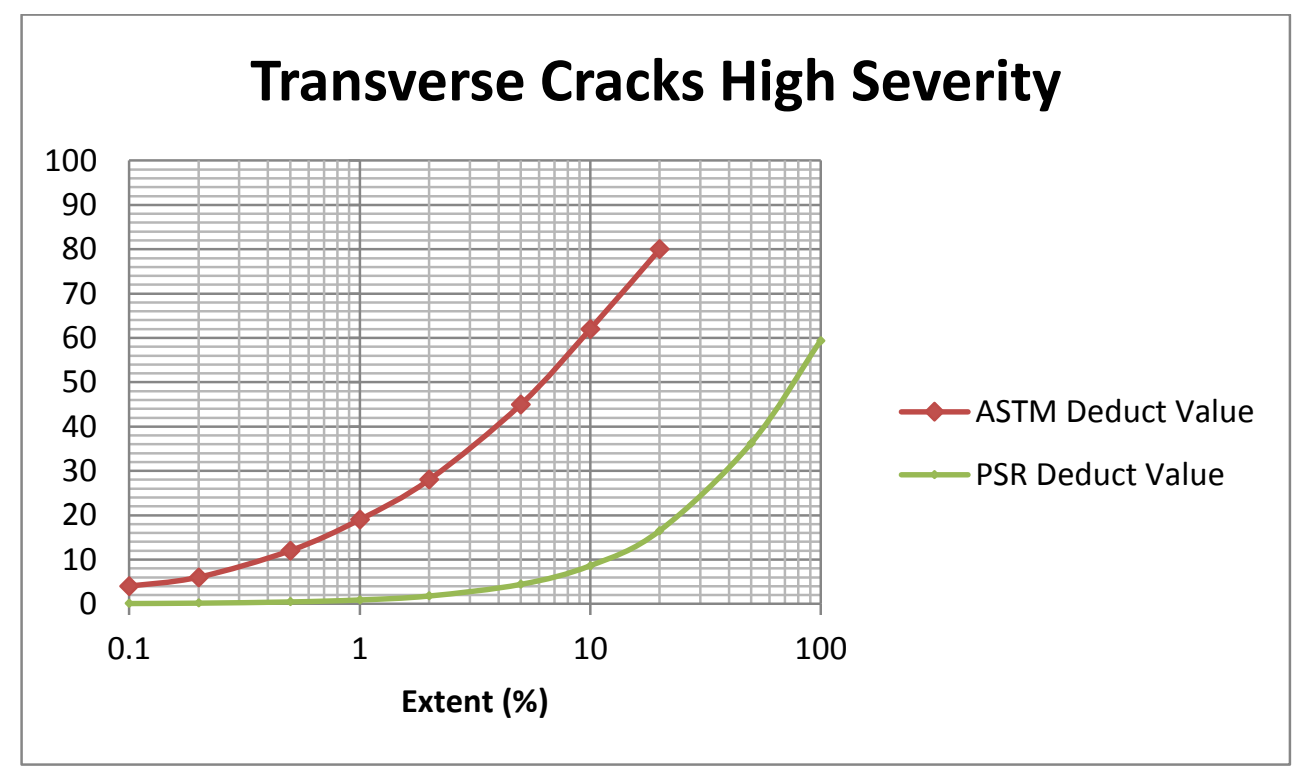

Figure 61: Transverse Cracks High Severity Deduct Curve Comparison

\section{Edge Cracking}

- $0.015,0.025,0.080$

- Severity definitions match closely.

- Initial DCR of 12:1 


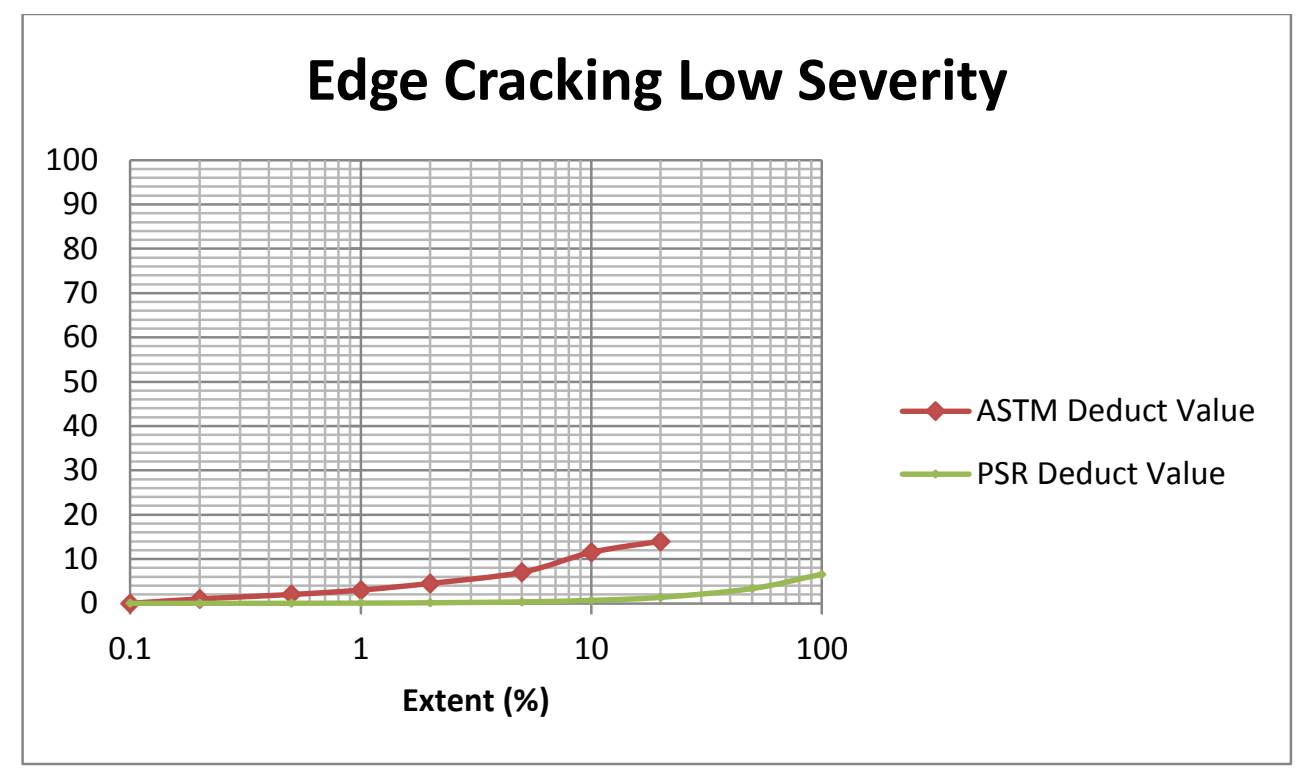

Figure 62: Edge Cracking Low Severity Deduct Curve Comparison

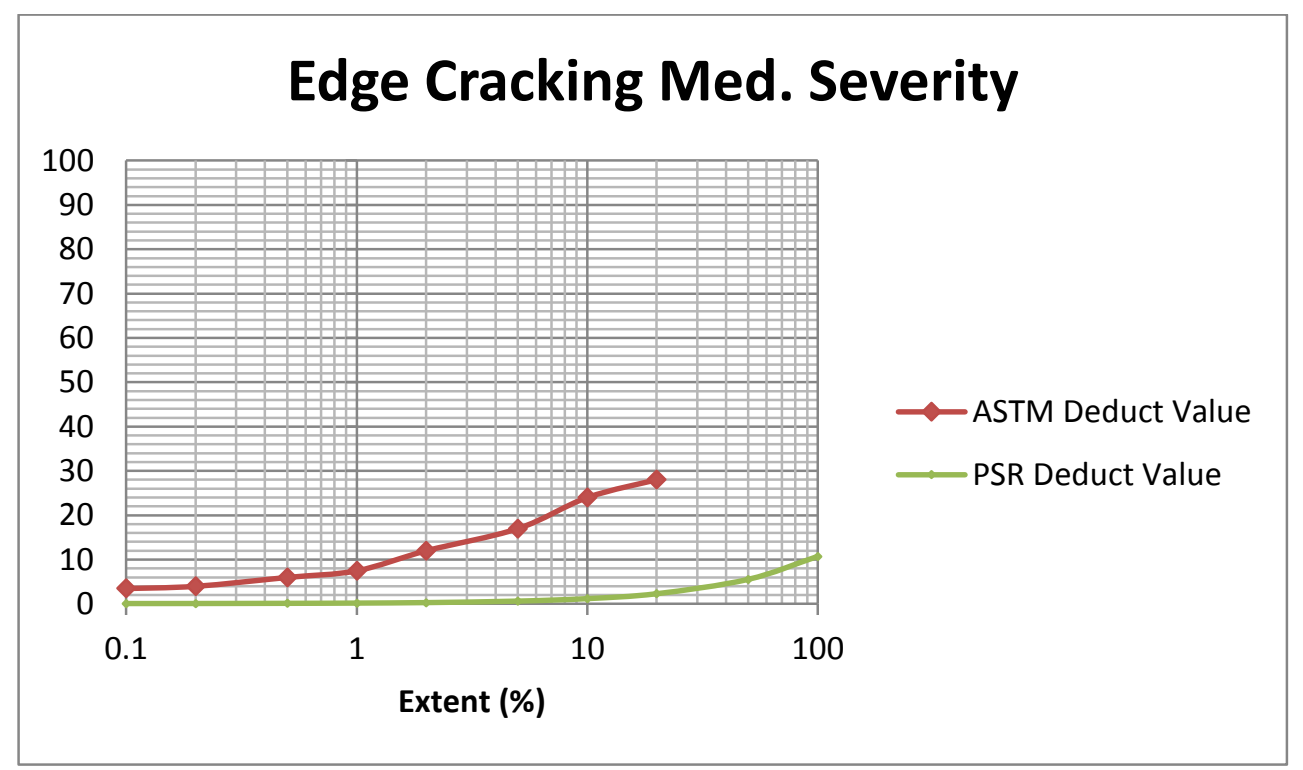

Figure 63: Edge Cracking Medium Severity Deduct Curve Comparison 


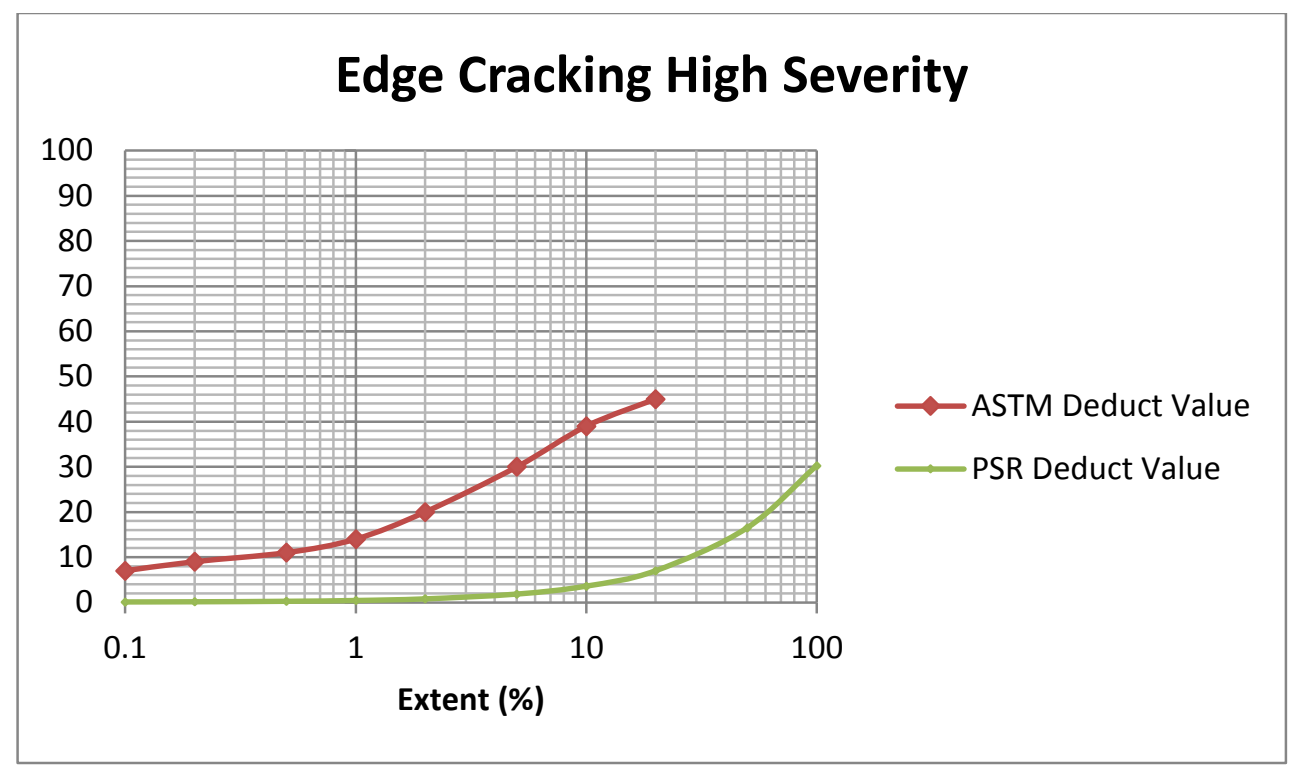

Figure 64: Edge Cracking High Severity Deduct Curve Comparison

\section{Alligator Cracking}

- $0.014,0.024,0.034$

- Severity definitions closely match.

- Initial DCR of approximately 2:1

- At high extents this ratio is surpasses and deemed acceptable due to the extreme nature of perceived distress associated with Alligator Cracking.

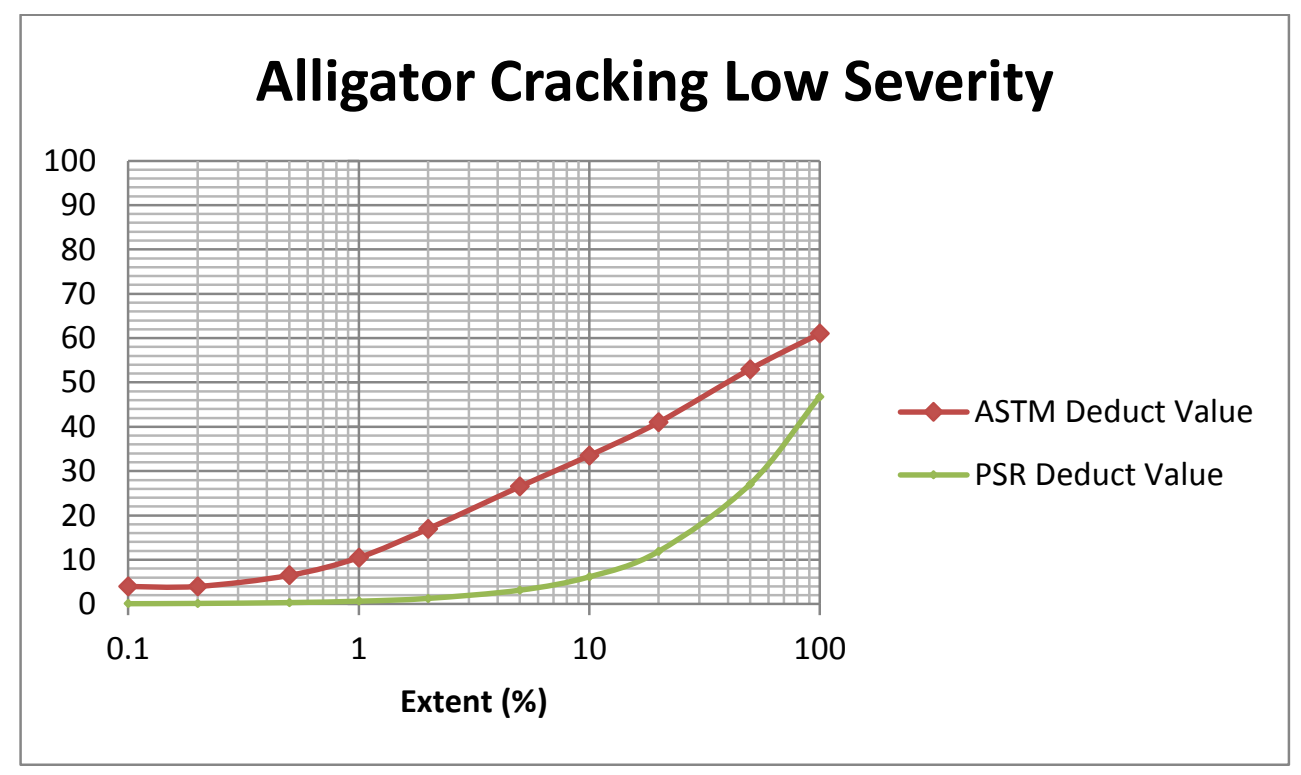

Figure 65: Alligator Cracking Low Severity Deduct Curve Comparison 


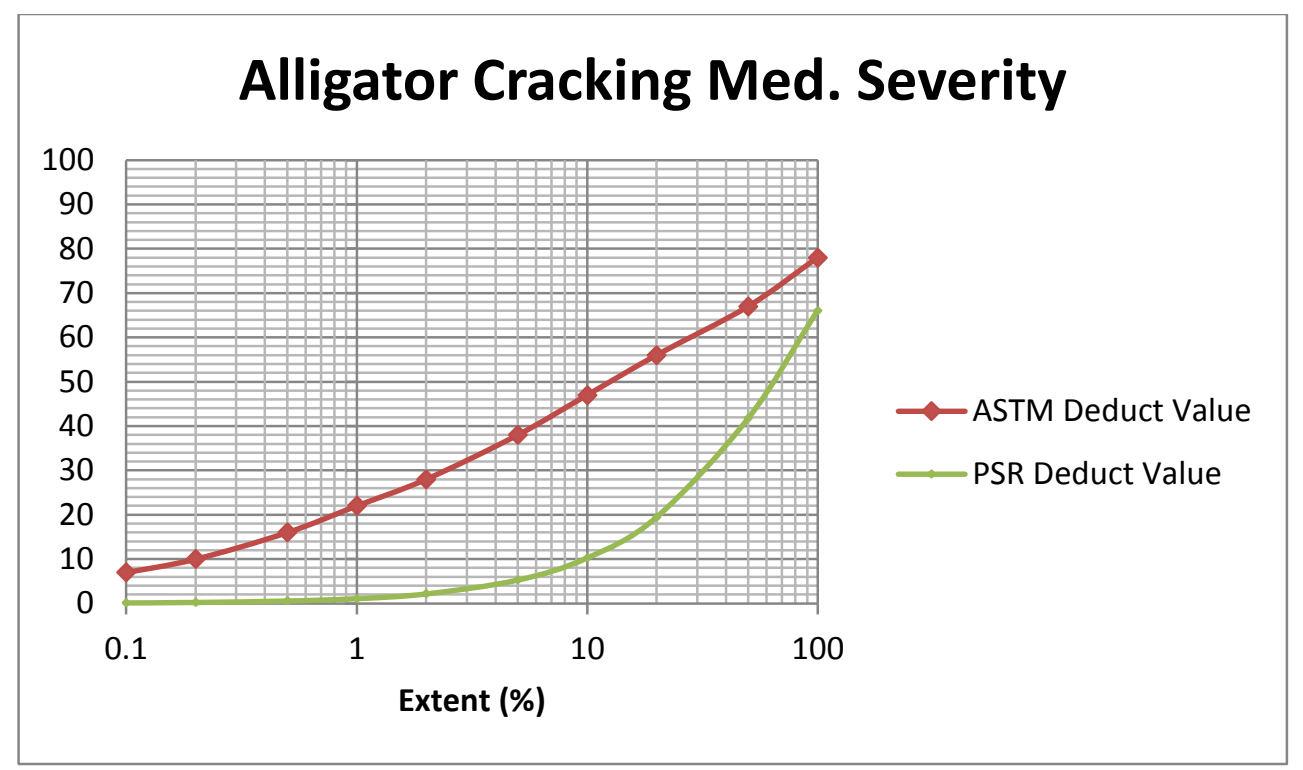

Figure 66: Alligator Cracking Medium Severity Deduct Curve Comparison

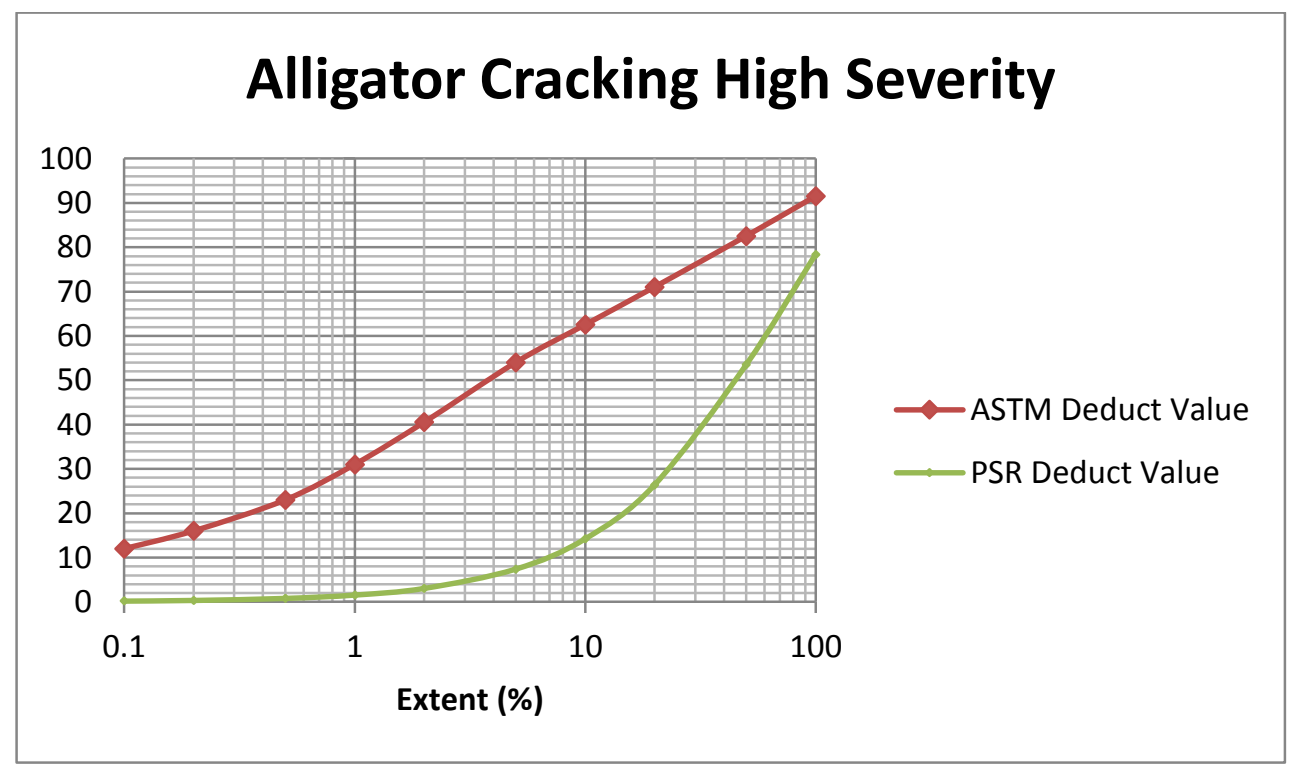

Figure 67: Alligator Cracking High Severity Deduct Curve Comparison

\section{Block Cracking}

- $0.055,0.105,0.165$

- Severity definitions are similar with the exception of crack width thresholds which are more lenient within the $\mathrm{PCl}$ definition, especially at High Severity. This necessitated a slight lowering of curves, especially in Medium and High Severity.

- Initial DCR of 1:1 
- A slight increase in all curves was also factored because several other distresses are included in Block Cracking and cannot be recorded if Block Cracking exists.

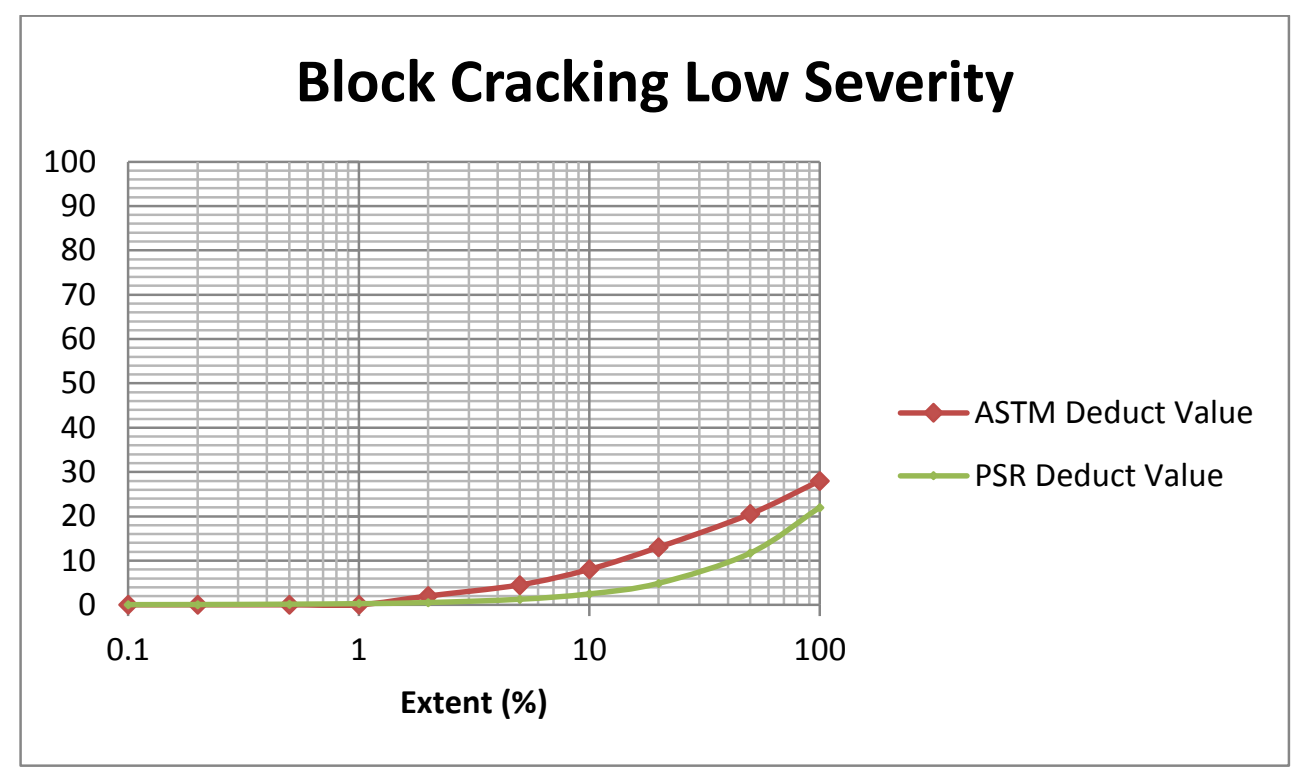

Figure 68: Block Cracking Low Severity Deduct Curve Comparison

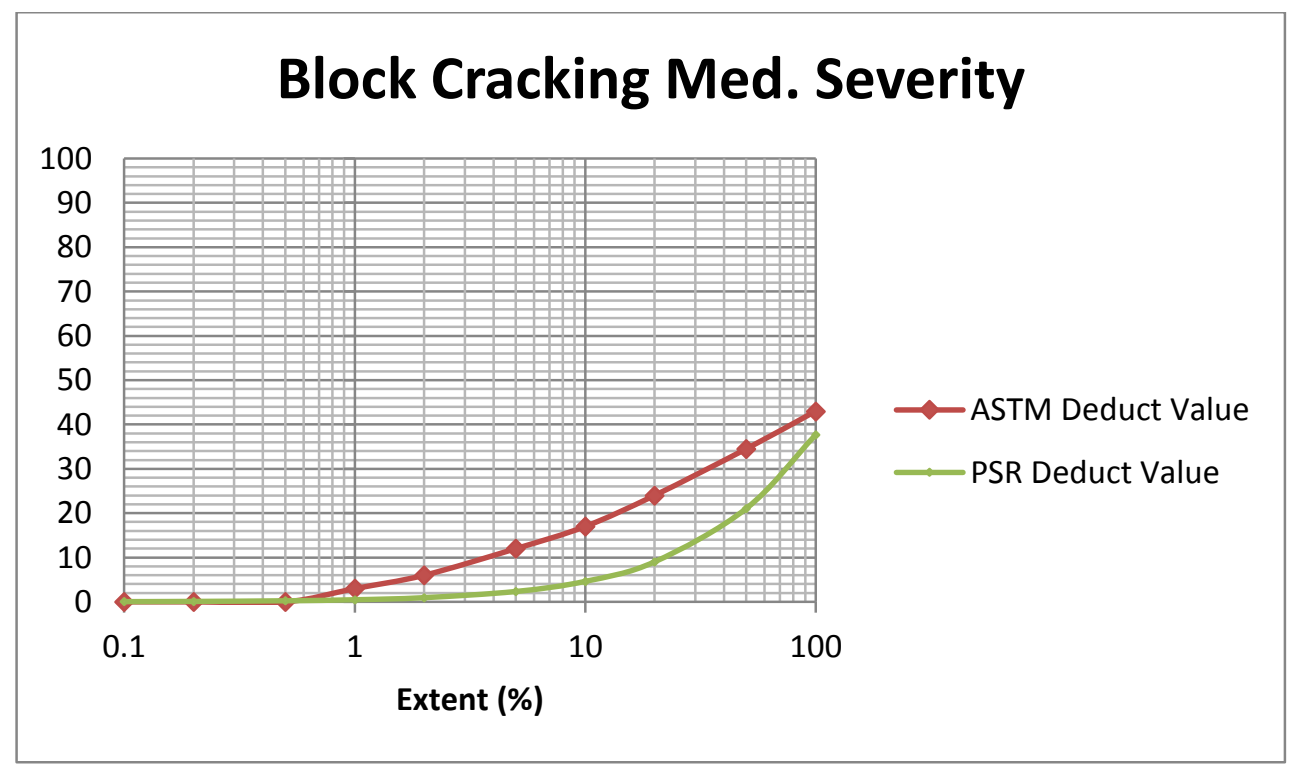

Figure 69: Block Cracking Medium Severity Deduct Curve Comparison 


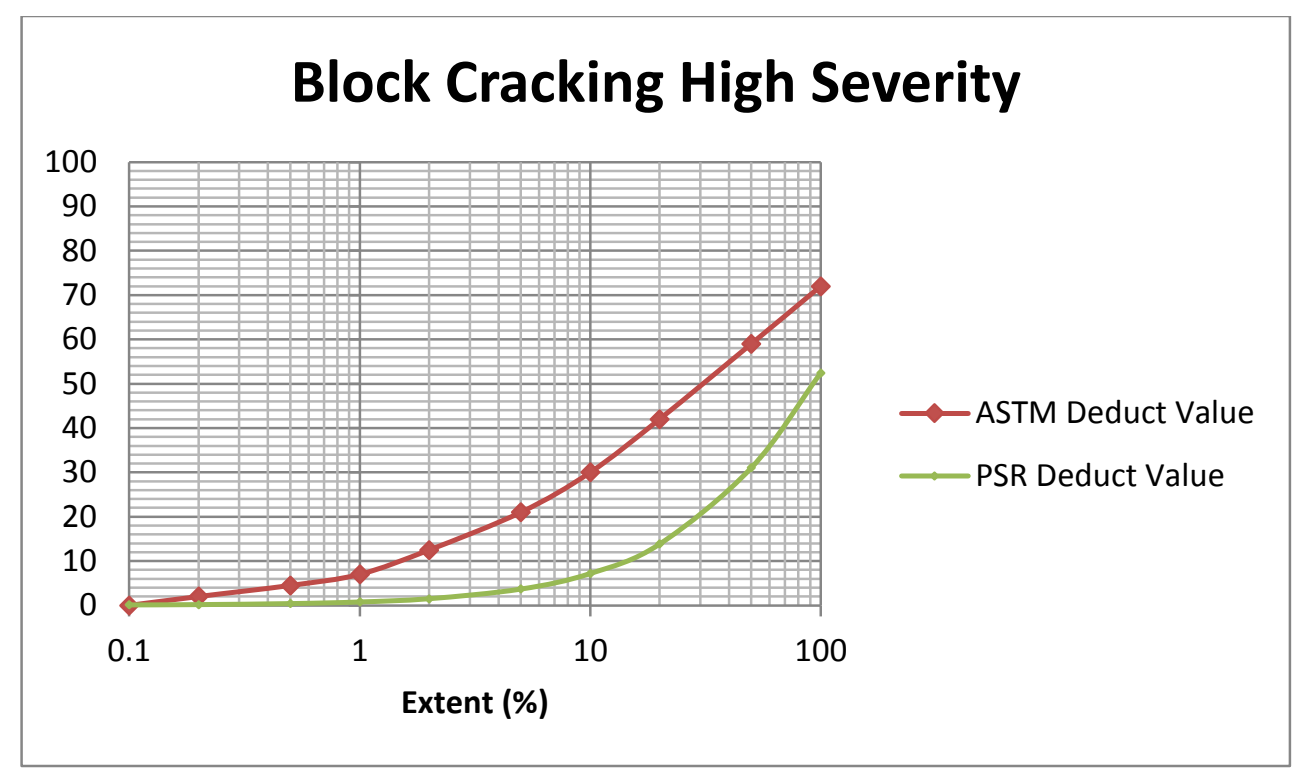

Figure 70: Block Cracking High Severity Deduct Curve Comparison

\section{Longitudinal Cracking}

- $\quad 0.020,0.030,0.060$

- Severity definitions are similar with the exception of crack width thresholds which are more lenient within the $\mathrm{PCl}$ definition, especially at High Severity. This necessitates a lowering of Medium and High Severity Curves

- Initial DCR of approximately 12:1

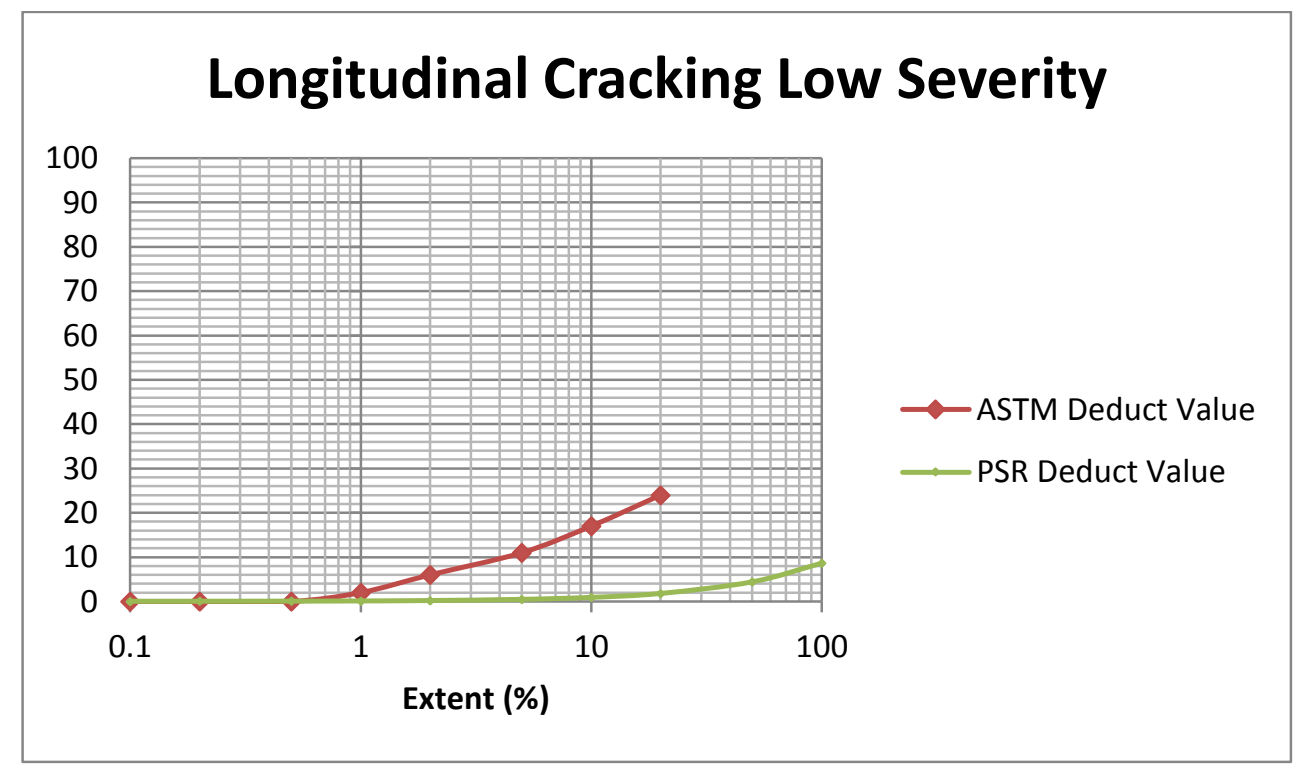

Figure 71: Longitudinal Cracking Low Severity Deduct Curve Comparison 


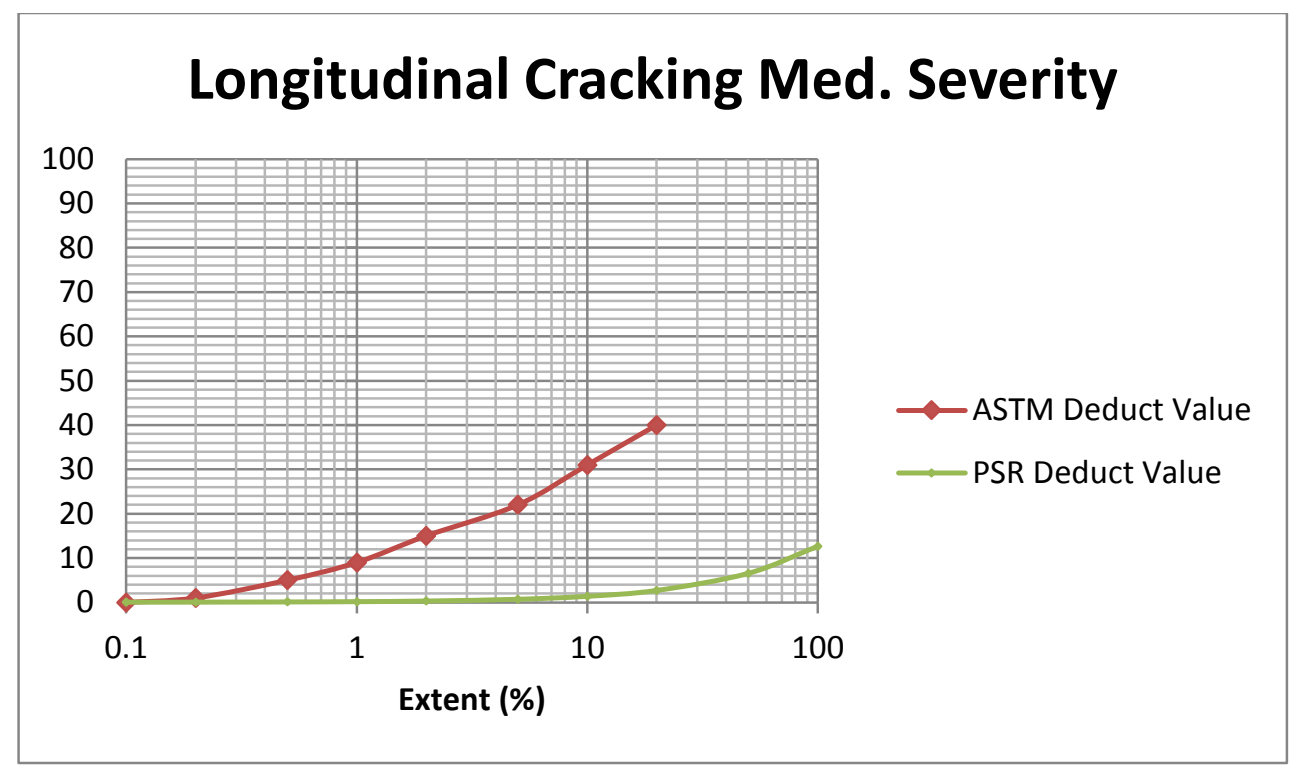

Figure 72: Longitudinal Cracking Medium Severity Deduct Curve Comparison

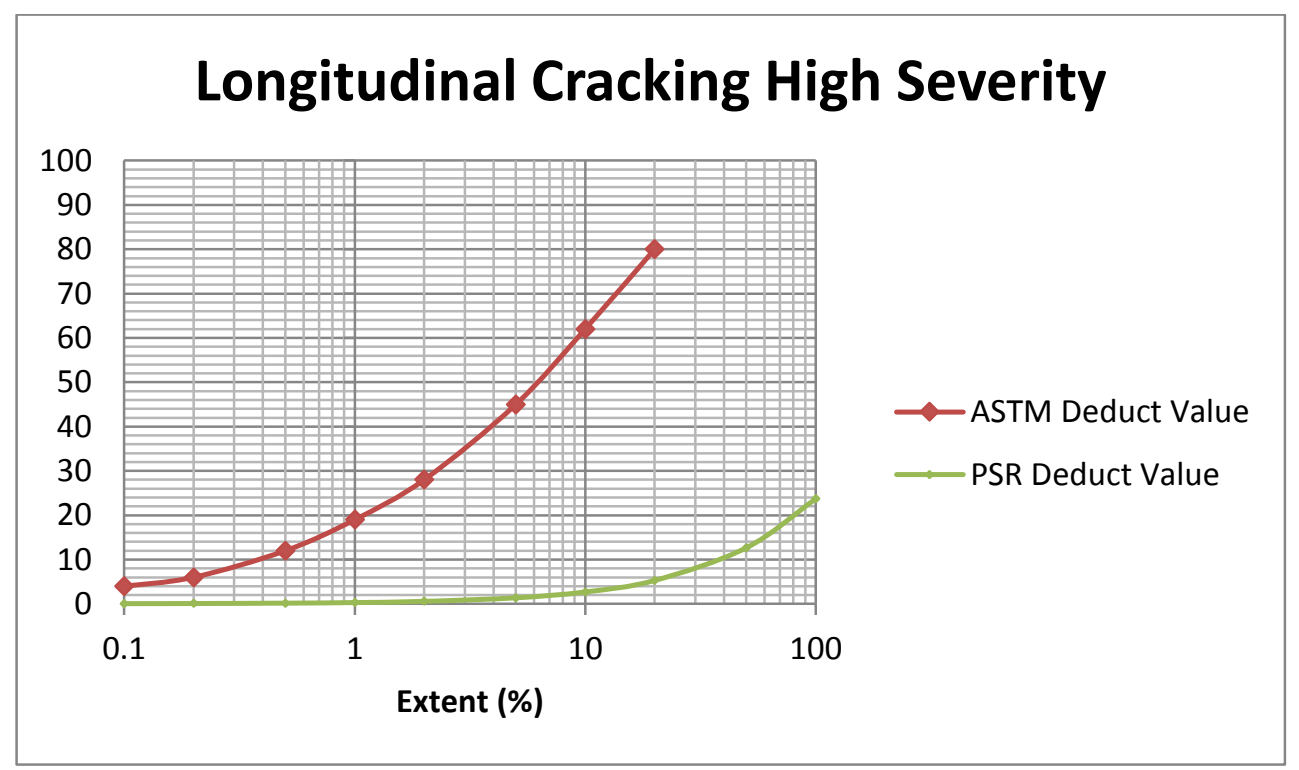

Figure 73: Longitudinal Cracking High Severity Deduct Curve Comparison

\section{Longitudinal Joint Deterioration}

- $0.025,0.045,0.080$

- For PCl, Longitudinal Joint Deterioration would be contained within the definitions of Longitudinal Cracking and Raveling to some degree.

- Weight factors are based on Longitudinal Cracking with a minor increase due to the nature of a paving joints, their susceptibility to deterioration, and potential affect on multiple lanes. 


\section{Lane Edge Cracking/Deterioration}

- $\quad 0.010,0.020,0.040$

- For PCl, Lane Edge Cracking/Deterioration would be contained within the definitions of Longitudinal Cracking and Raveling to some degree.

- Weight factors are based on Longitudinal Cracking with a minor decrease this time due to the fact that Lane Edges are driven across far less often than cracking present within or between travel lanes.

\section{Raveling/Weathering}

- 0.040

- $\quad \mathrm{PCl}$ includes Weathering at Low, Medium, and High Severities. PSR Raveling/Weathering matches the definition of Medium Severity Weathering most closely so this was the comparison made.

- Initial DCR of 1:1

- The curve was decreased because Weathering generally occurs in older pavements and is accompanied by other distresses.

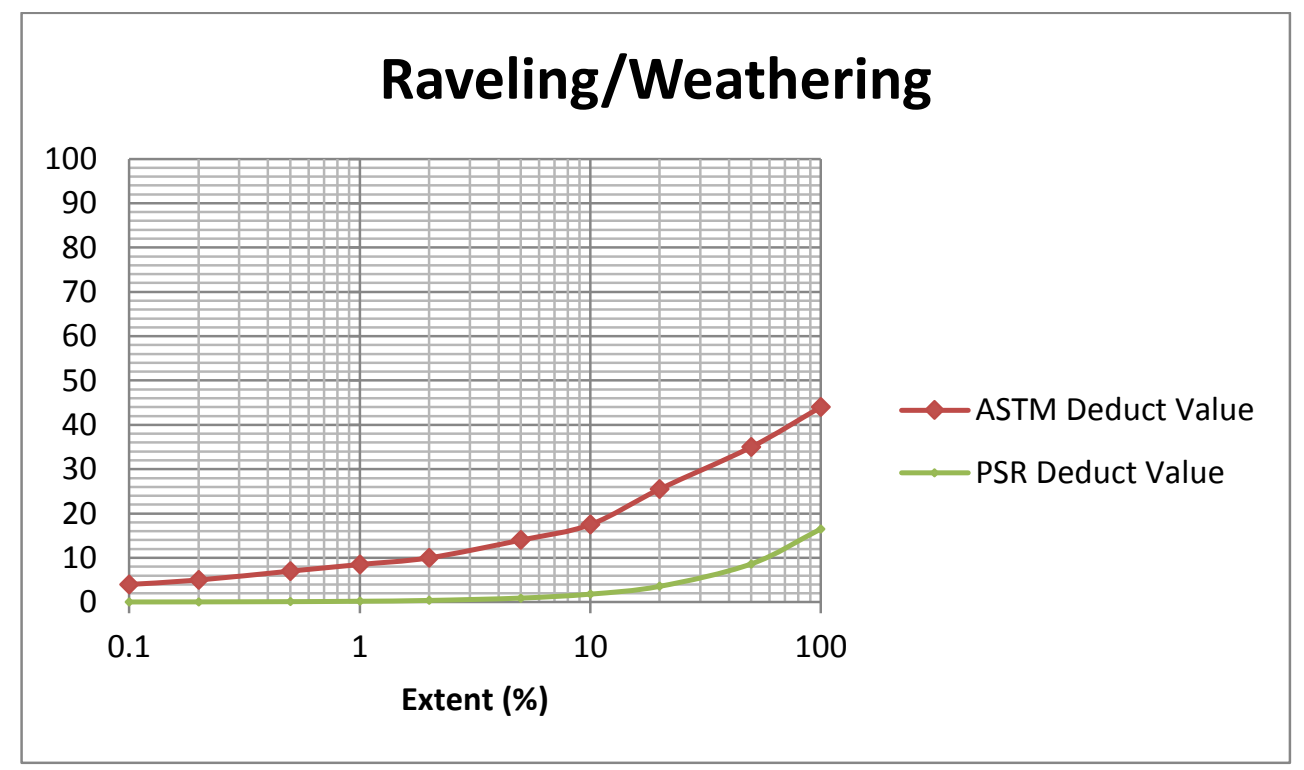

Figure 74: Raveling/Weathering Deduct Curve Comparison

\section{Patching}

- 0.030

- PSR definition matches PCI Low Severity Patching definition so it was used for comparison. PCI also includes Medium and High Severity Patching which are related to distress found within patching. PSR allows for the recording of distresses within patching so this would be redundant.

- Initial DCR of 1:1

- Curve was reduced because well maintained Patching should have a minimal effect on perceived distress. Additionally, distresses within Patches are already counted separately. 


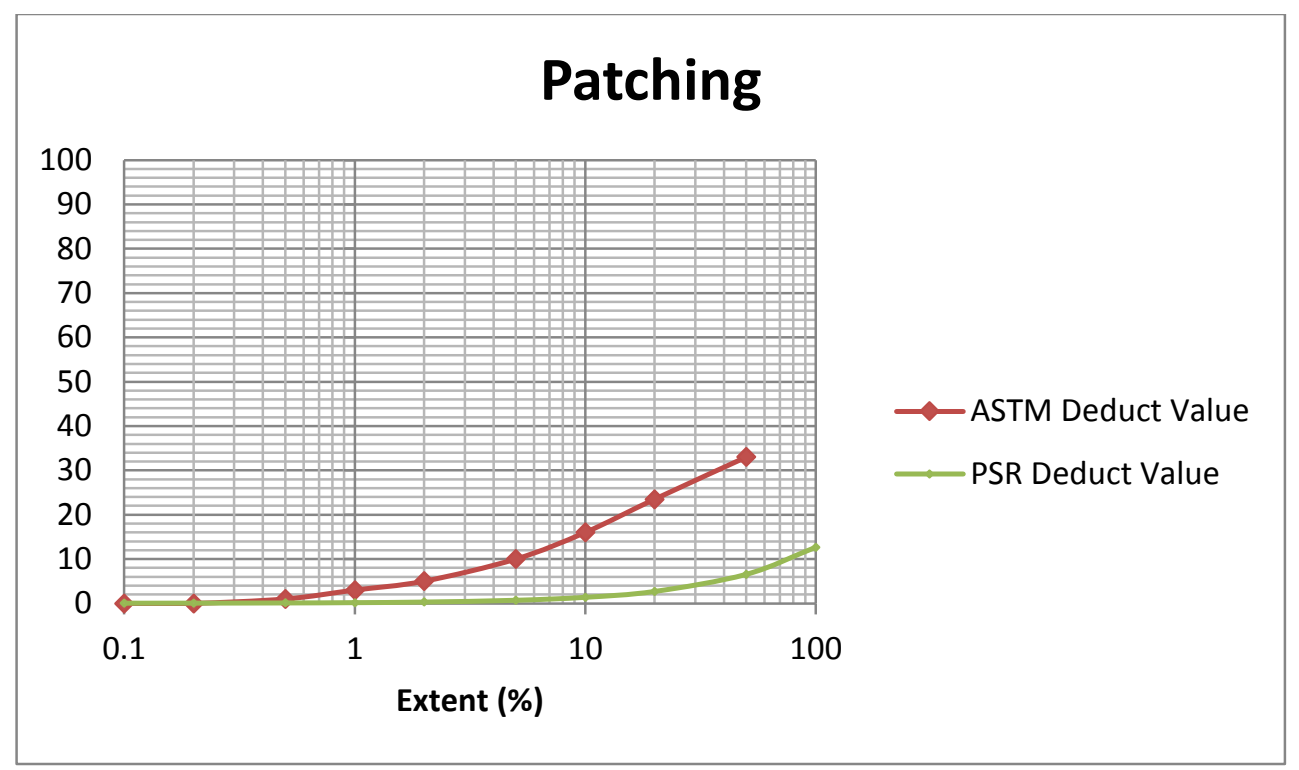

Figure 75: Patching Deduct Curve Comparison

\section{Rutting}

- $0.065,0.150$

- PCl includes Low, Medium, and High Severities. PSR Low and High Severity definitions closely match PCI Low and Medium Severities respectively so these were used for comparison.

- Rutting is either recorded as 0 or 100 percent in PSR so those points are the only that matter when matching curves.

- Initial DCR of 2:1

- At high extents of High Severity Rutting this ratio is surpassed and deemed acceptable due to the extreme nature of perceived distress associated with Rutting. 


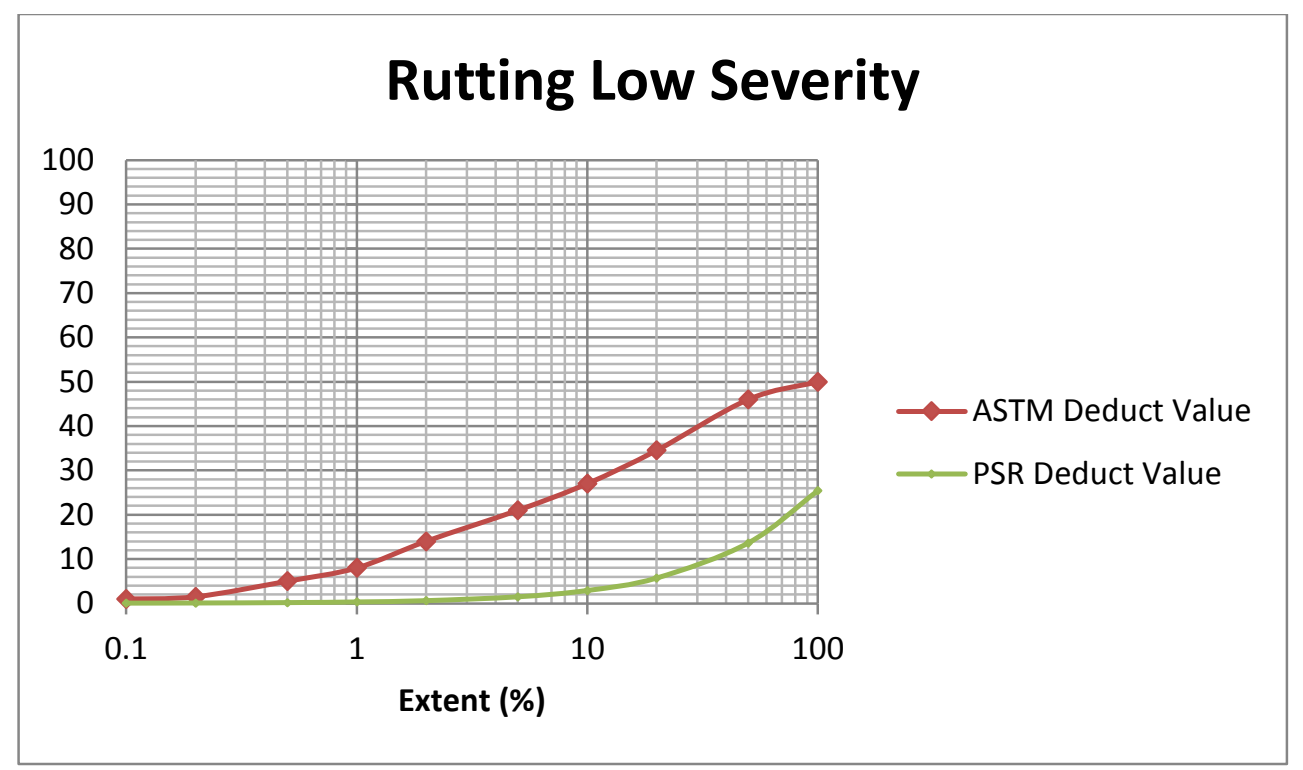

Figure 76: Rutting Low Severity Deduct Curve Comparison

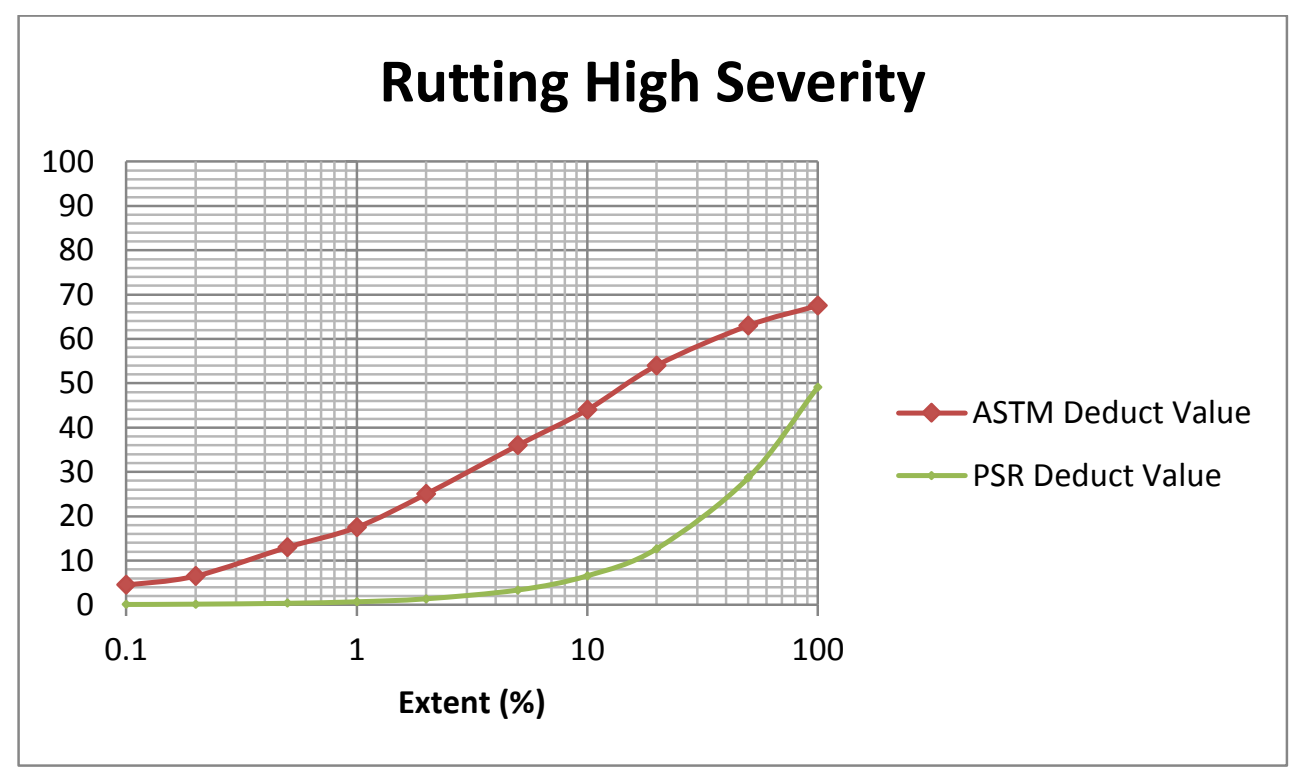

Figure 77: Rutting High Severity Deduct Curve Comparison

\section{B.2 Deduct Comparison for Concrete Surfaces}

\section{Transverse Cracks}

- $0.040,0.100,0.210$

- PSR Transverse Cracks were compared to PCI Linear Cracking. Definitions are generally similar in crack identification. 
- Large reduction was made because $\mathrm{PCl}$ combines Transverse and Longitudinal Cracking in Linear Cracking.

- $\mathrm{PCl}$ counts Linear Cracking extent on an affected slab basis which through experience severely overestimates distress associated with Transverse Cracks on relatively long slabs which necessitated the separation.

- Due to a change in PSR Transverse Crack extent calculation which was consistent with asphalt surface pavements, a significant increase was necessitated.

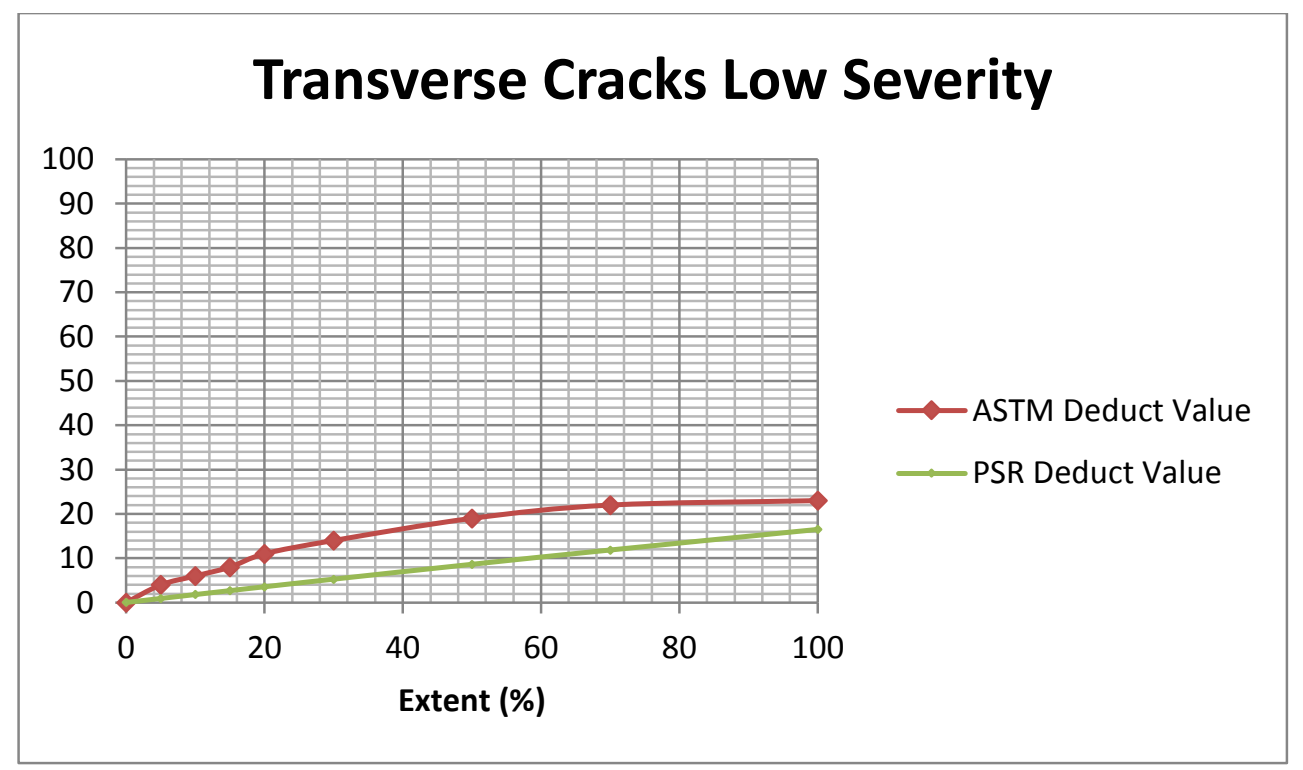

Figure 78: Transverse Cracks Low Severity Deduct Curve Comparison

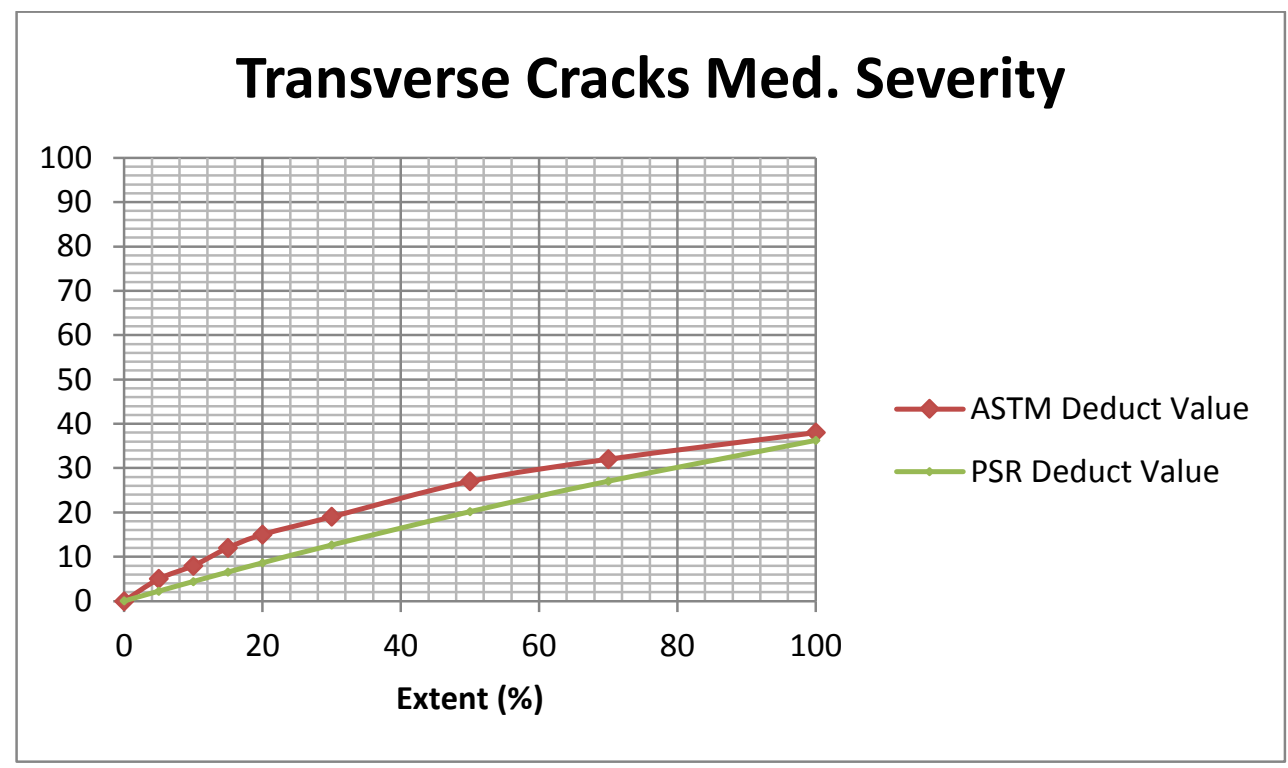

Figure 79: Transverse Cracks Medium Severity Deduct Curve Comparison 


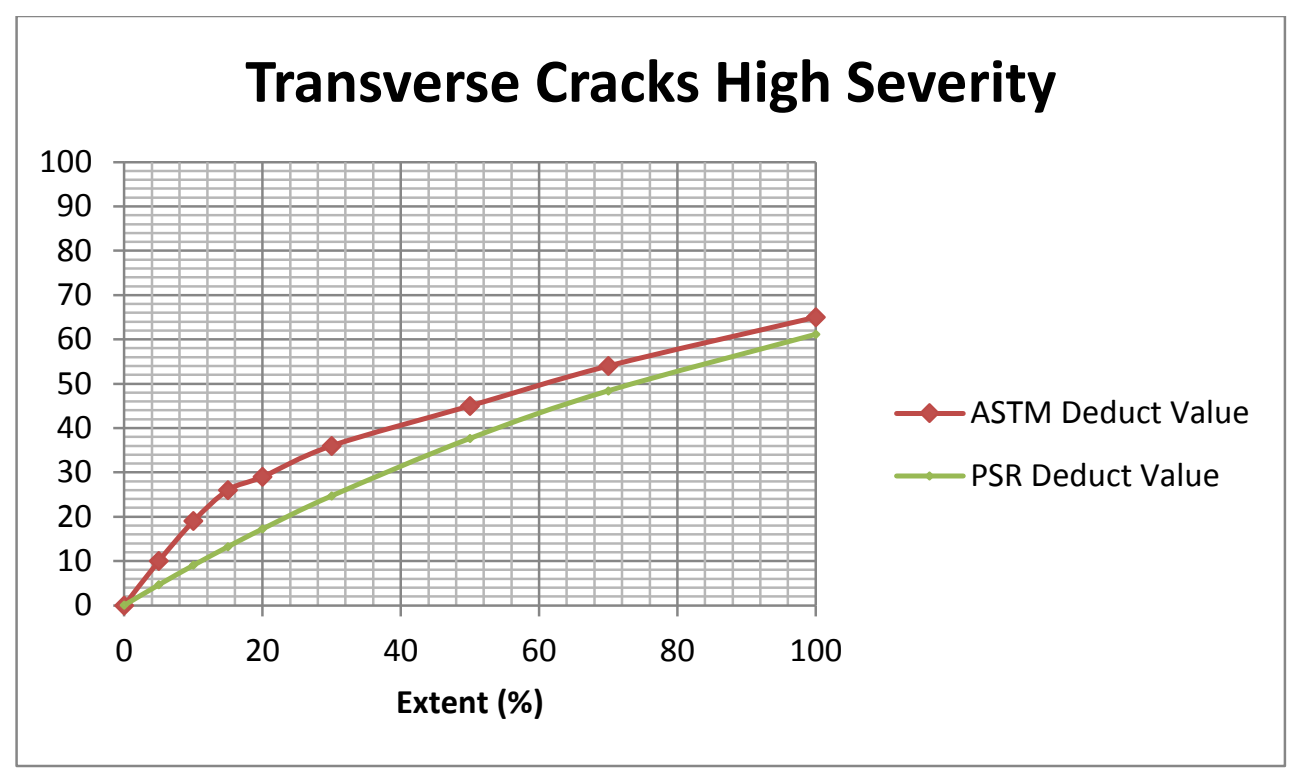

Figure 80: Transverse Cracks High Severity Deduct Curve Comparison

\section{Transverse Joint Spalls}

- $0.020,0.075,0.150$

- Transverse Joint Spalls closely match the definition for PCI Joint Spalls so these curves were used for comparison.

- Slight Reduction made to all Severity Transverse Joint Spalls because Transverse and Longitudinal Spalling are separated now. This was a small reduction because from experience most spalling will occur in transverse joints.

- At the request of WVDOH, Low and Medium Severity were increased to the initial curve location because from experience, small spalls in transverse joints deteriorate quickly toward High Severity. When compared to PCI this could mean that PSR will slightly overestimate distress associated with Low or Medium Transverse Joint Spalls but in this case it is necessary as an indicator of imminent future distress. 


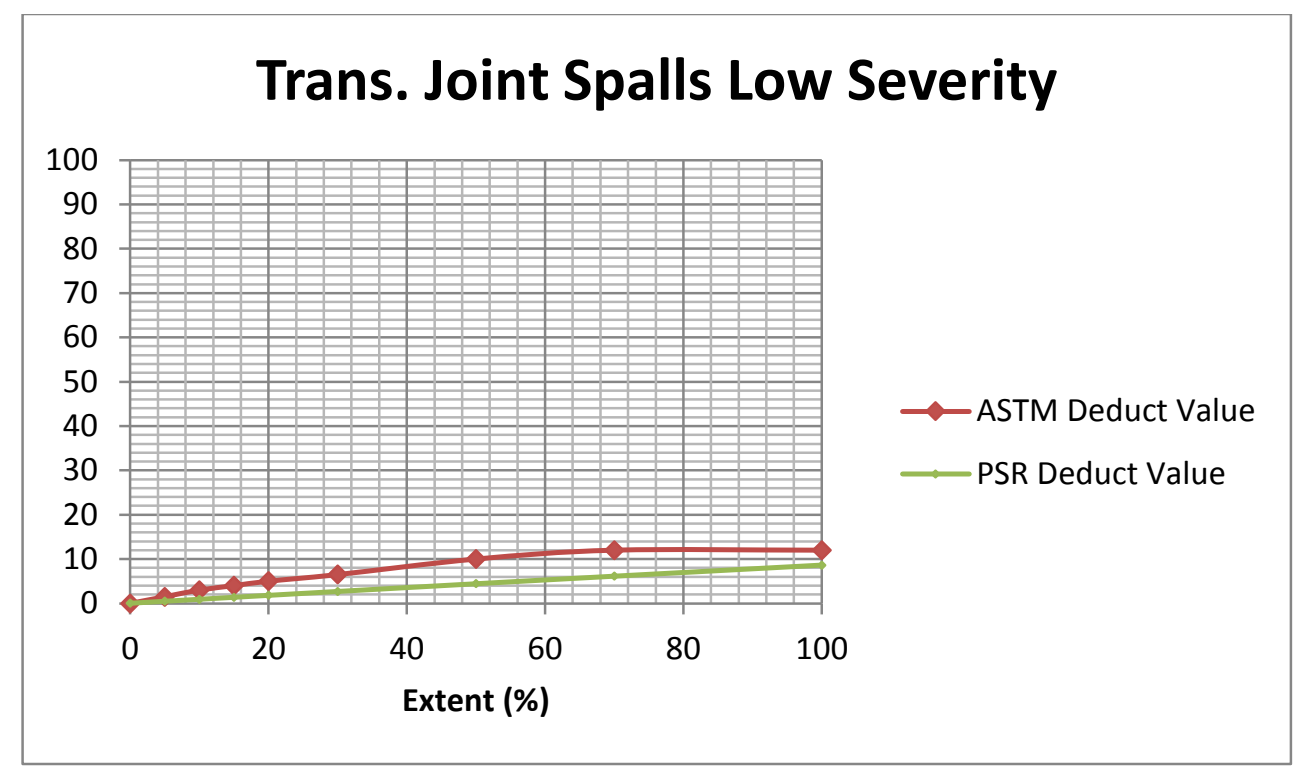

Figure 81: Transverse Joint Spalls Low Severity Deduct Curve Comparison

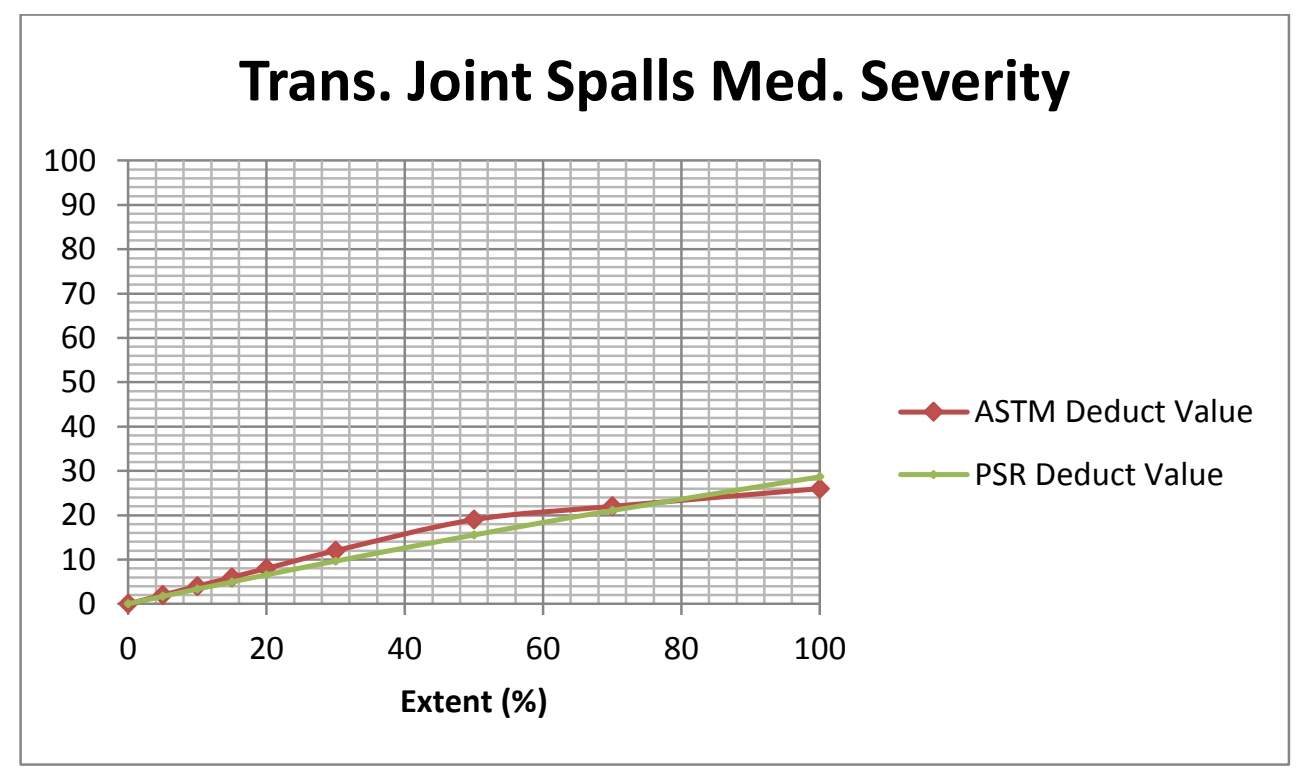

Figure 82: Transverse Joint Spalls Medium Severity Deduct Curve Comparison 


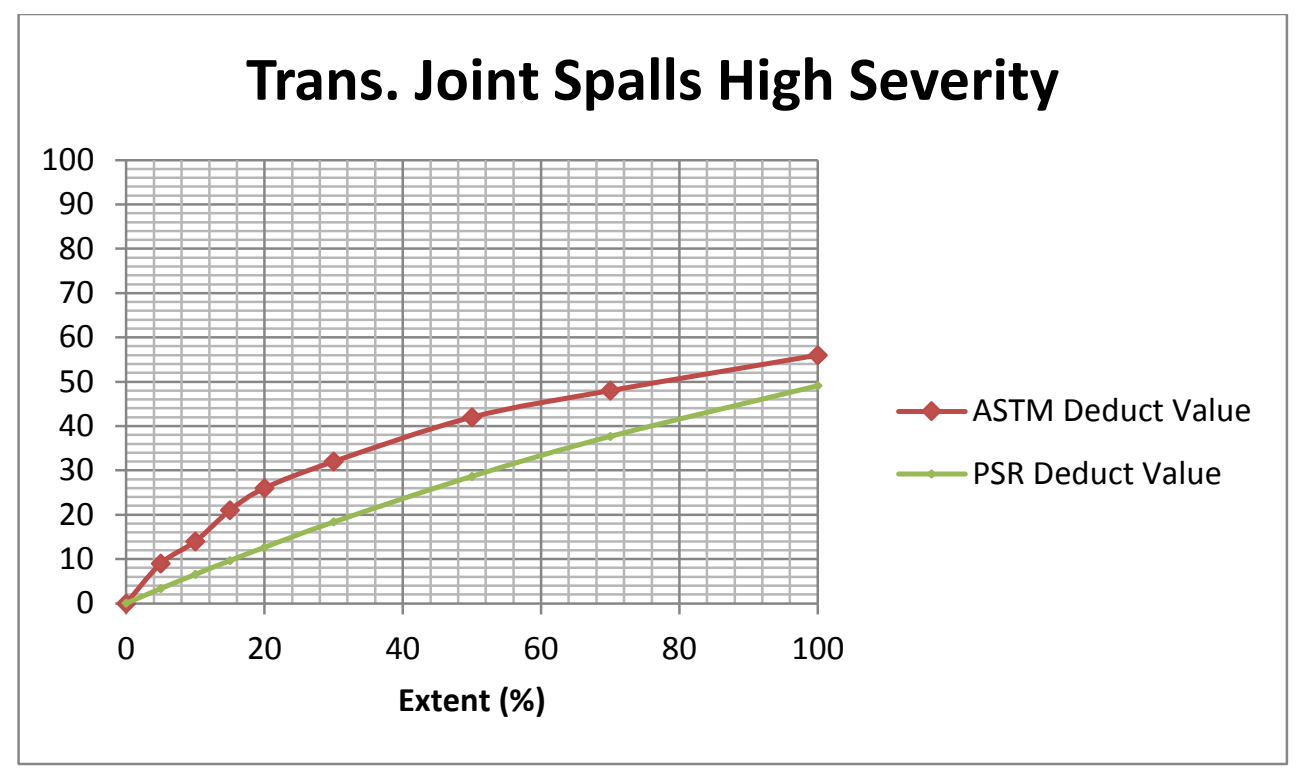

Figure 83: Transverse Joint Spalls High Severity Deduct Curve Comparison

\section{Transverse Joint Faults}

- $0.000,0.110,0.210$

- PSR Transverse Joint Faults generally matches the PCI Faulting definitions so this was the comparison made.

- The Low Severity Faulting weight factor was reduced to zero at the request of WVDOH because the minimum threshold for recording a Fault was so small that a severe overestimation of distress appeared to be made on test sections with large amounts of nearly insignificant faults present. These faults are still recorded for warranty purposes.

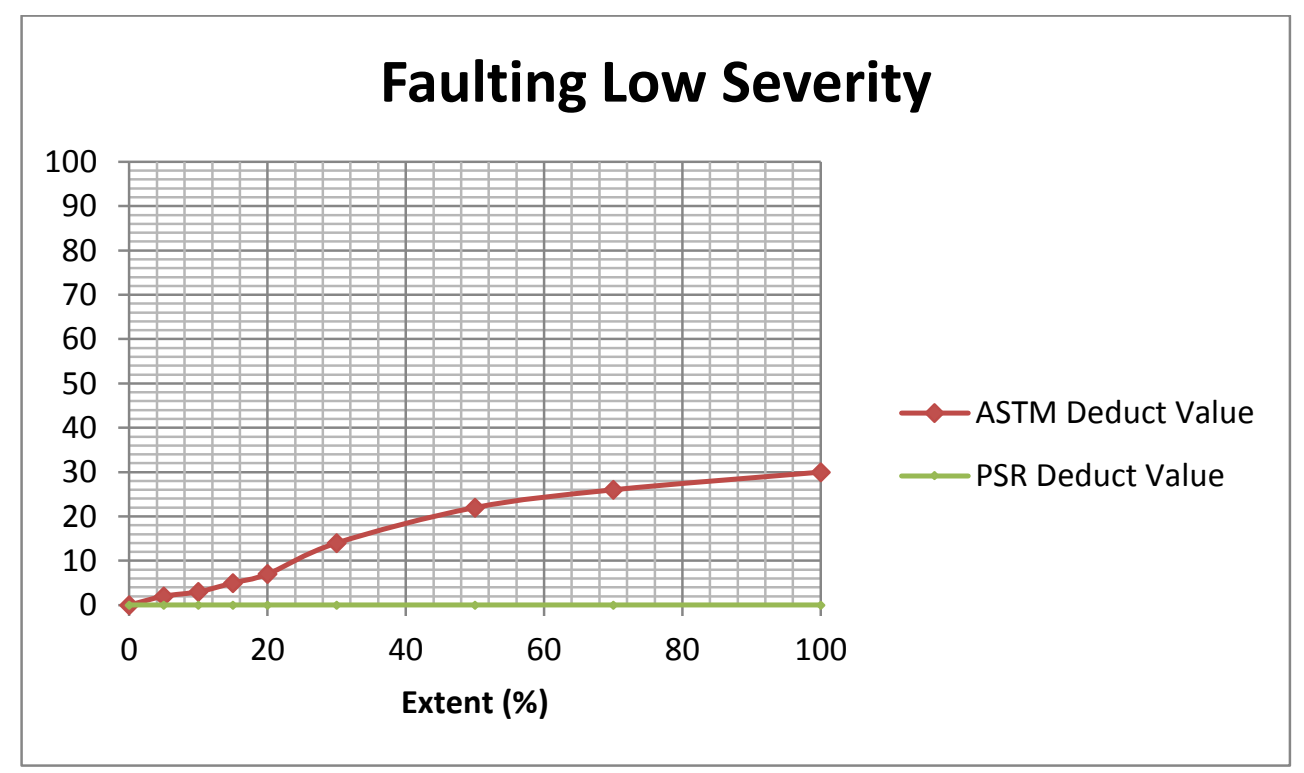

Figure 84: Faulting Low Severity Deduct Curve Comparison 


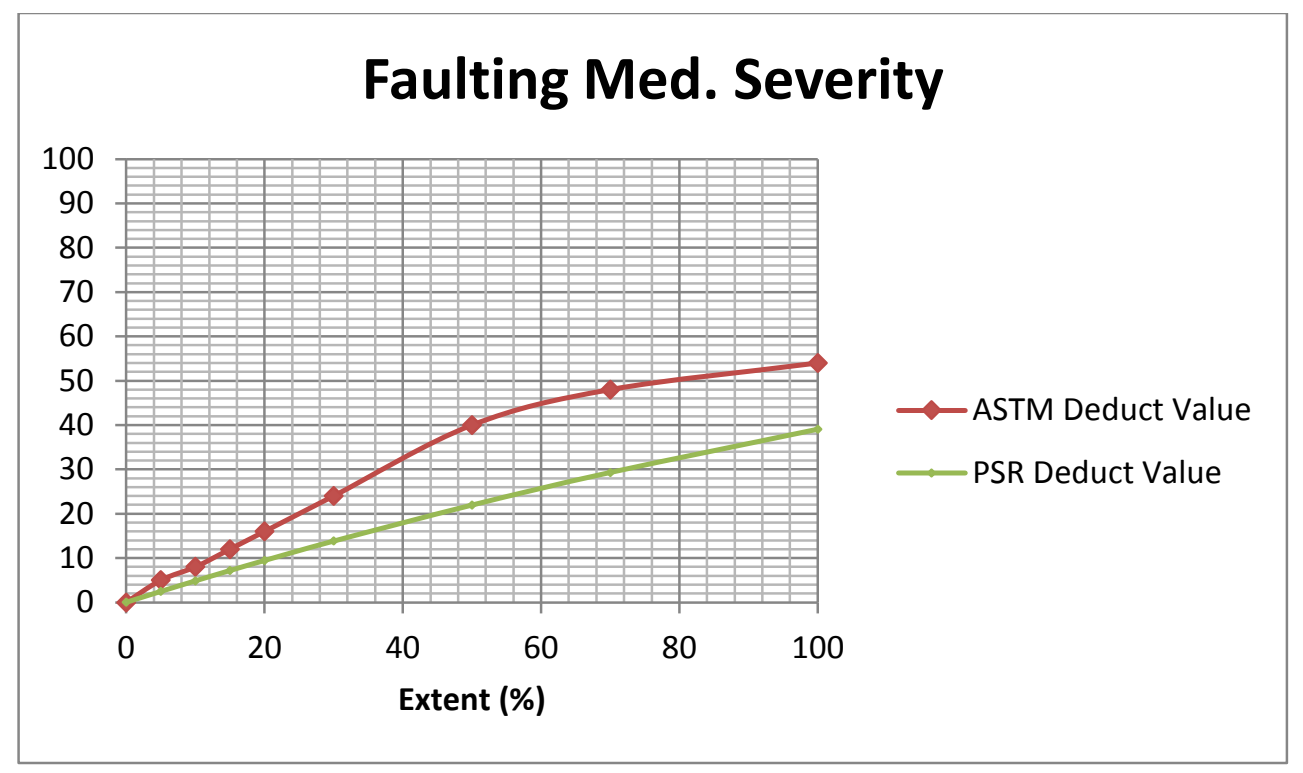

Figure 85: Faulting Medium Severity Deduct Curve Comparison

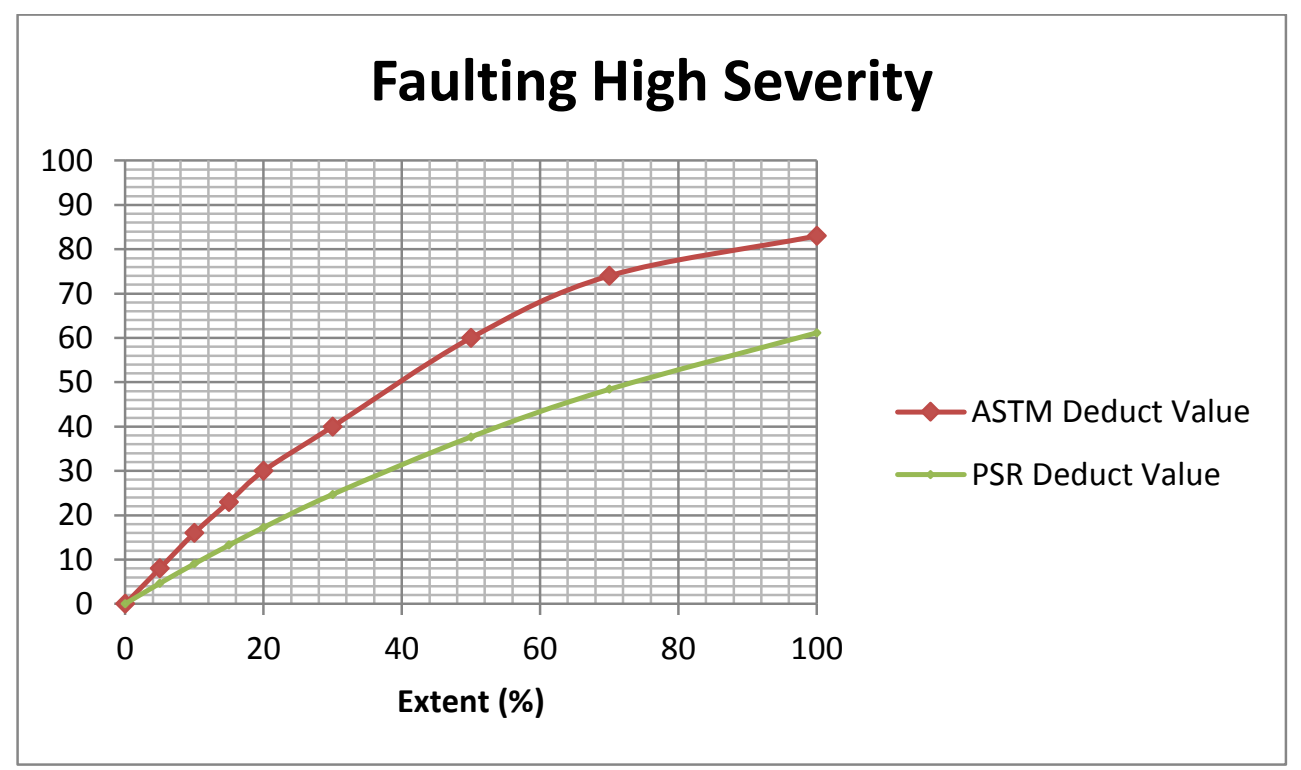

Figure 86: Faulting High Severity Deduct Curve Comparison

\section{Blowups}

- $\quad 0.250$

- PSR Blowups definition most closely matched PCI Medium Severity Blowups so this was the comparison made.

- Because Blowups recorded in PSR are counted at this weight factor, when compared to distress associated with $\mathrm{PCl}$ defined Blowups, PSR will overestimate small Blowups and underestimate large Blowups. To maintain simplicity, this was deemed acceptable for PCl defined Low Severity 
Blowups based on the extreme nature of perceived distress associated with Blowups. $\mathrm{PCl} \mathrm{High}$ Severity Blowups are so devastating that this difference was of little effect.

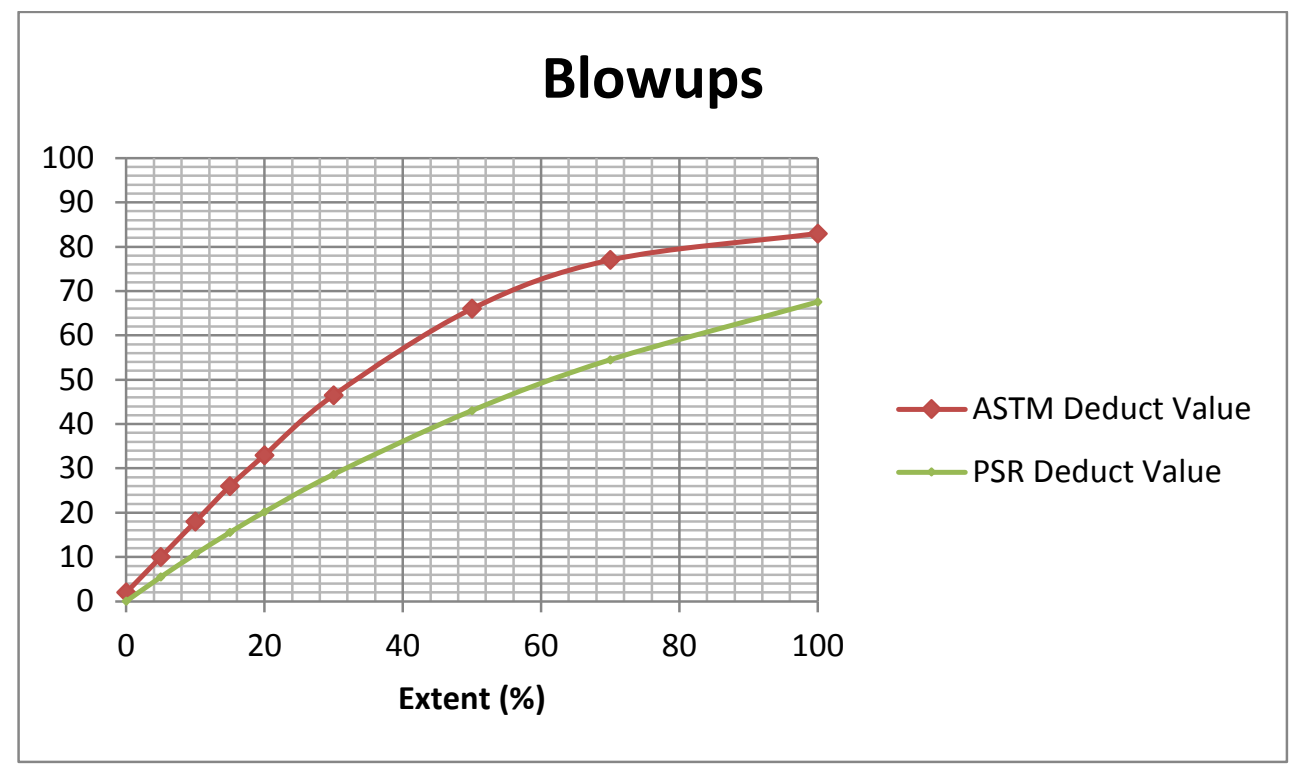

Figure 87: Blowups Deduct Curve Comparison

\section{Longitudinal Cracking}

- $\quad 0.030,0.090,0.190$

- PSR Longitudinal Cracking was compared to PCI Linear Cracking. Definitions are generally similar in crack identification.

- Large reduction was made because $\mathrm{PCl}$ combines Transverse and Longitudinal Cracking in Linear Cracking.

- $\quad \mathrm{PCl}$ counts Linear Cracking extent on an affected slab basis which through experience severely overestimates distress associated with Longitudinal Cracks which affect less than a full slab length on relatively long slabs which necessitated the separation.

- Due to a change in PSR Longitudinal Crack extent calculation which was consistent with asphalt surface pavements, a significant increase was necessitated. 


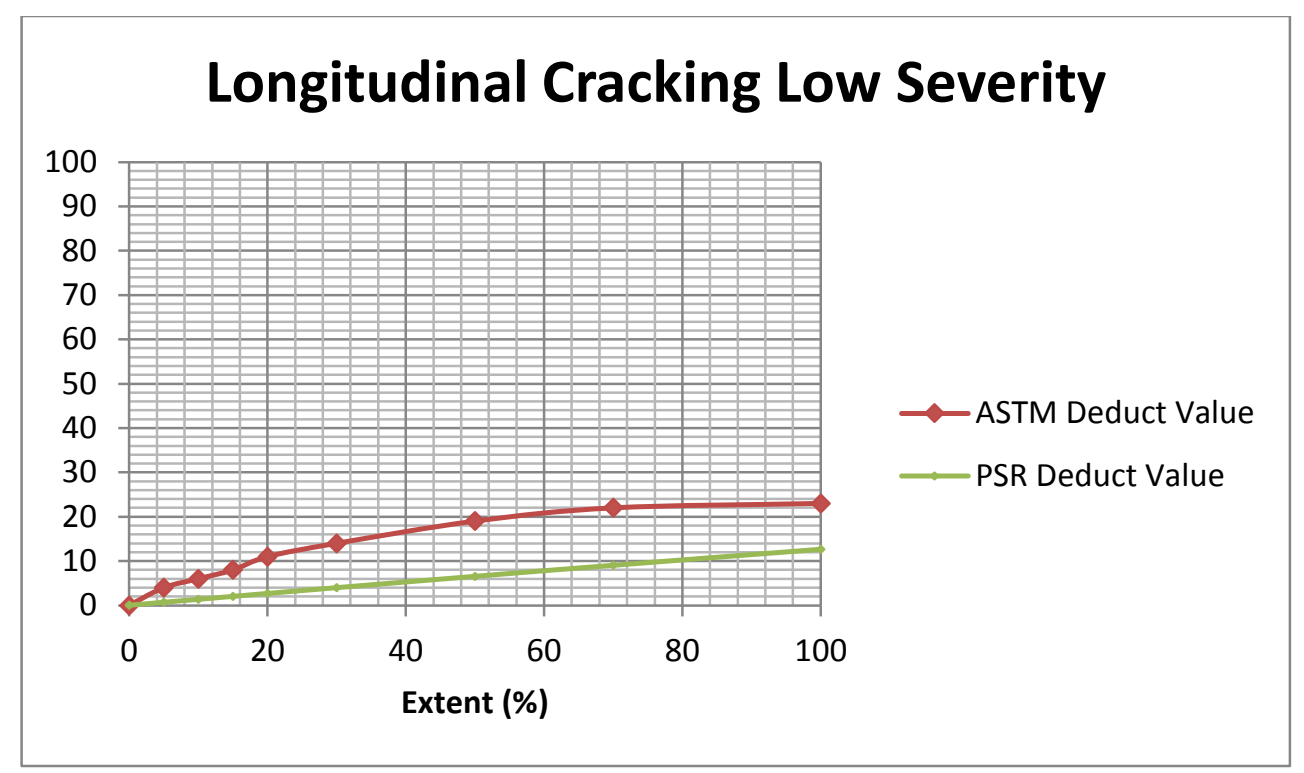

Figure 88: Longitudinal Cracking Low Severity Deduct Curve Comparison

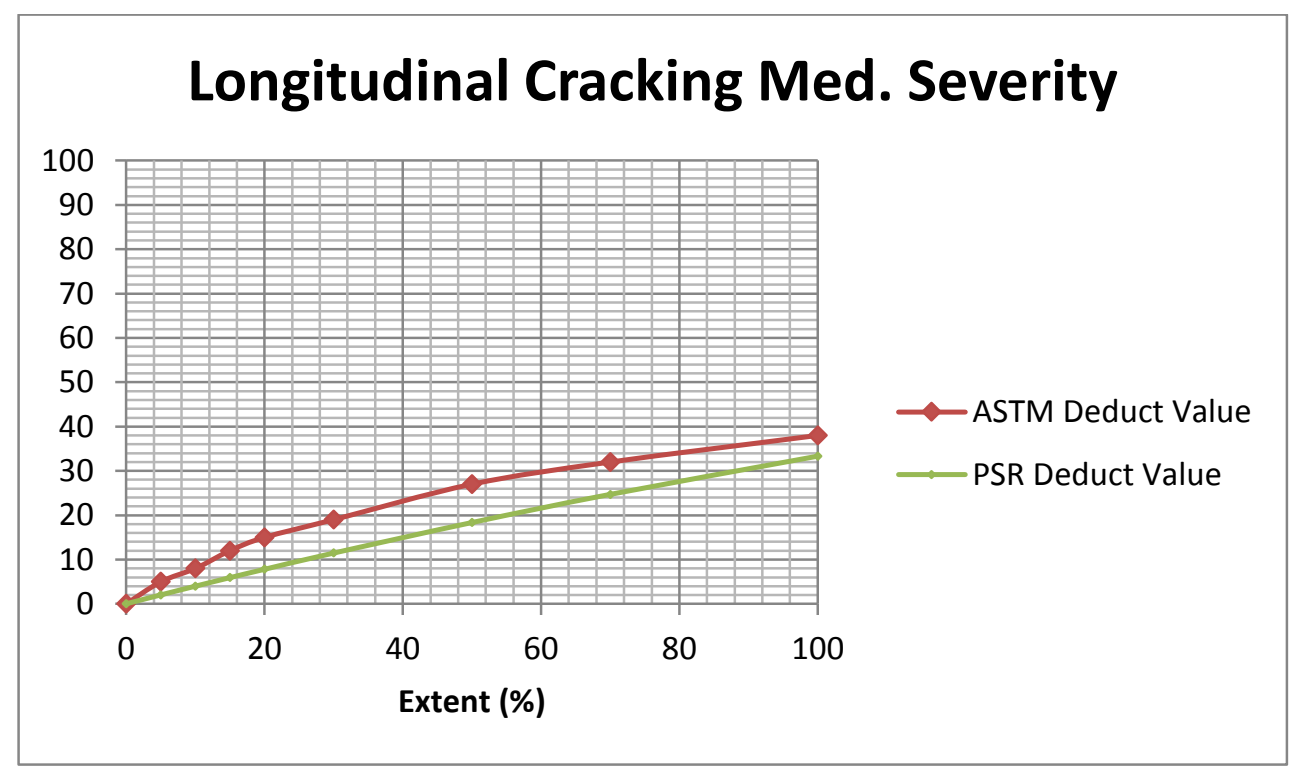

Figure 89: Longitudinal Cracking Medium Severity Deduct Curve Comparison 


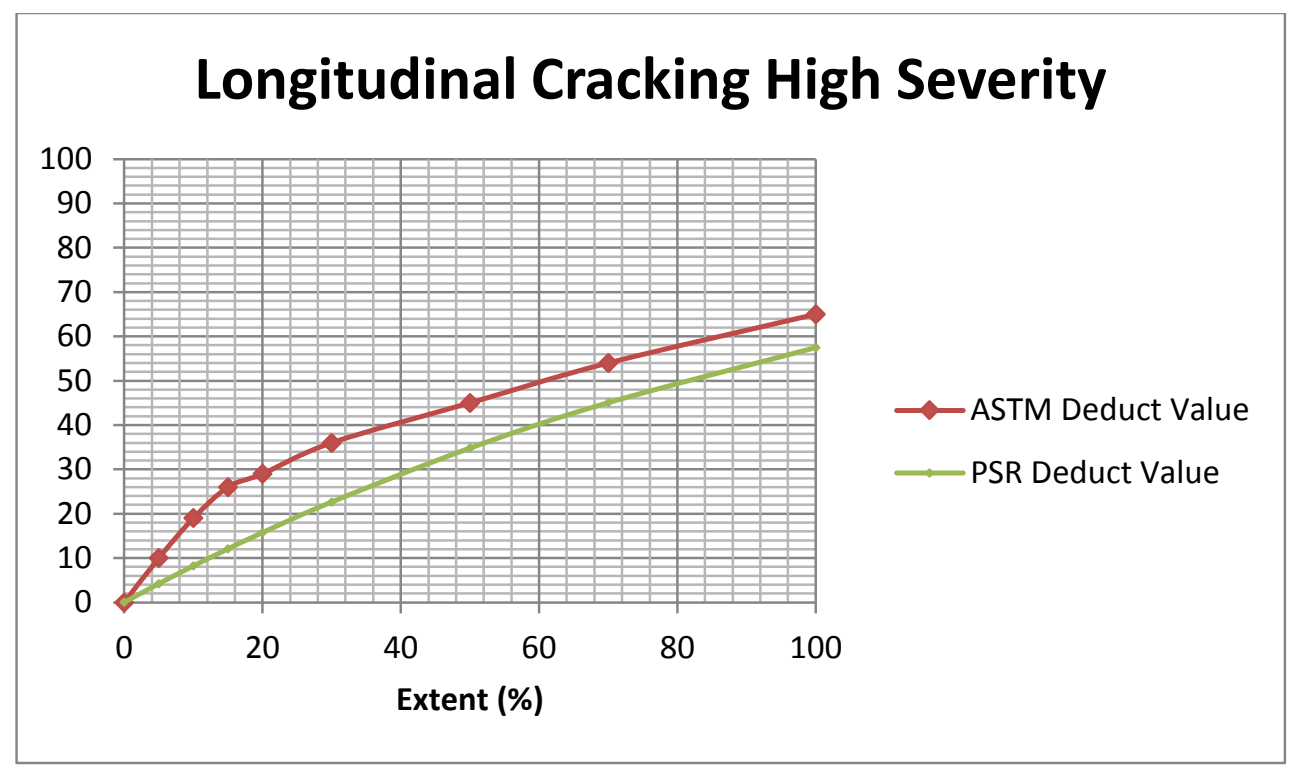

Figure 90: Longitudinal Cracking High Severity Deduct Curve Comparison

\section{Longitudinal Joint Spalling}

- $0.020,0.060,0.150$

- Longitudinal Joint Spalling is based on PCl Joint Spalling where severity definitions generally match.

- The graphs of the PCI Deduct Curves are from Transverse Joint Spalling Deduct Curve Comparisons.

- A small reduction to Medium Severity was made because joints between travel lanes are driven across far less than transverse joints.

- No reduction was made to Low Severity because the deduct value is so insignificant that a proportional reduction was not noticeable.

- No reduction was made to High Severity because of the potential for loss of material included within the definition which could affect perceived distress in the travel lane.

\section{Lane Edge Spalling}

- $0.010,0.050,0.150$

- Lane Edge Spalling is based on PCI Joint Spalling where severity definitions generally match.

- The graphs of the PCI Deduct Curves are from Transverse Joint Spalling Deduct Curve Comparisons.

- An additional reduction to Low and Medium Severity curves was made due to the fact that Lane Edges are driven across far less often than cracking present within or between travel lanes.

- This reduction was not made for High Severity because the definition includes the potential for loss of material into the lane that could affect perceived distress in the travel lane. 


\section{D-Cracking}

- 0.100

- PSR D-Cracking definition most closely matched PCI Medium Severity D-Cracking so this was the comparison made.

- Because D-Cracking recorded in PSR are counted at this weight factor, when compared to distress associated with PCl defined D-Cracking, PSR will overestimate Low D-Cracking and underestimate High D-Cracking. To maintain simplicity, this was deemed acceptable for PCI defined Low Severity D-Cracking based on the extreme nature of perceived distress associated with D-Cracking. PCl High Severity D-Cracking is devastating enough that this difference was of little effect.

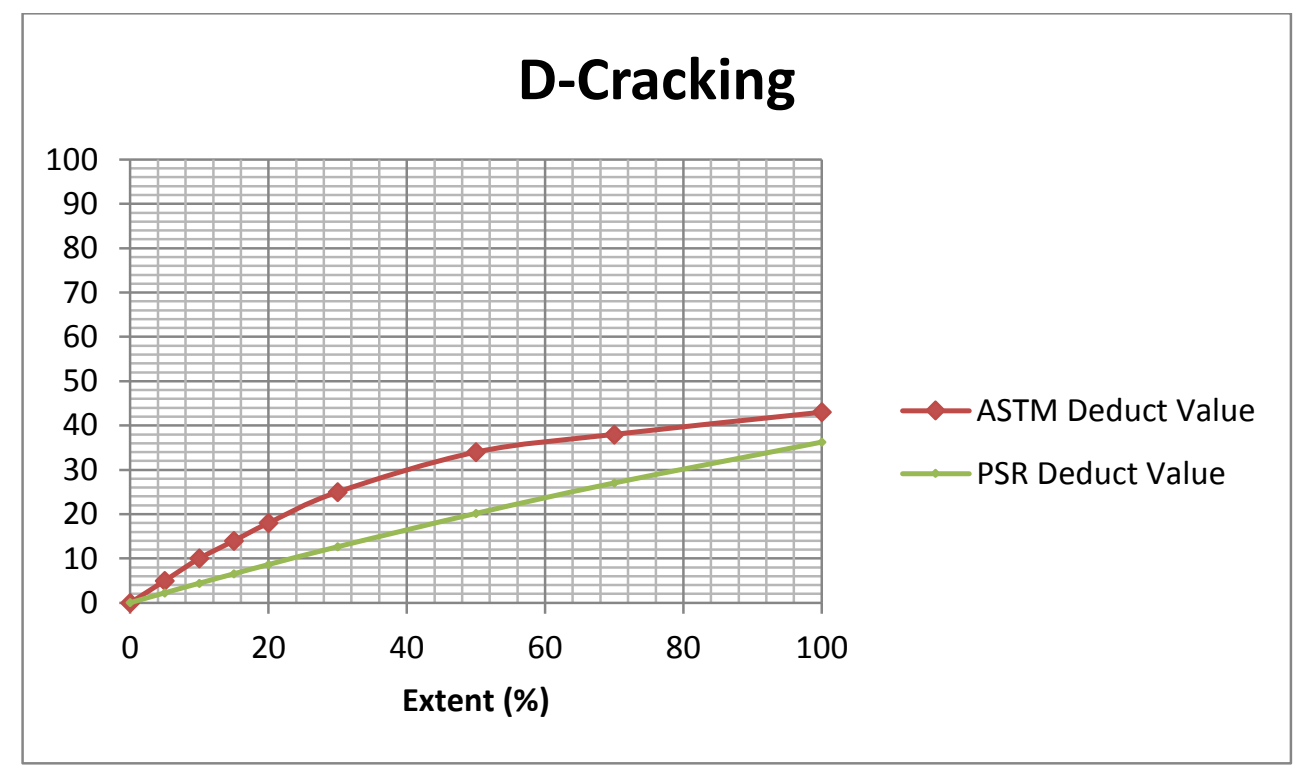

Figure 91: D-Cracking Deduct Curve Comparison

\section{Scaling}

- .020

- $\quad \mathrm{PCl}$ defines Low, Medium, and High Severity Scaling. PSR minimum definition of Scaling most closely matches PCI Low Severity Scaling so this was used for comparison.

- Little reduction was made to the curve to account for the potential of a surface with no other distresses where no correction factor would be used in $\mathrm{PCl}$.

- Without Medium and High Severity Scaling the potential for Scaling exceeding the Low Severity threshold necessitated no additional reduction of the curve. 


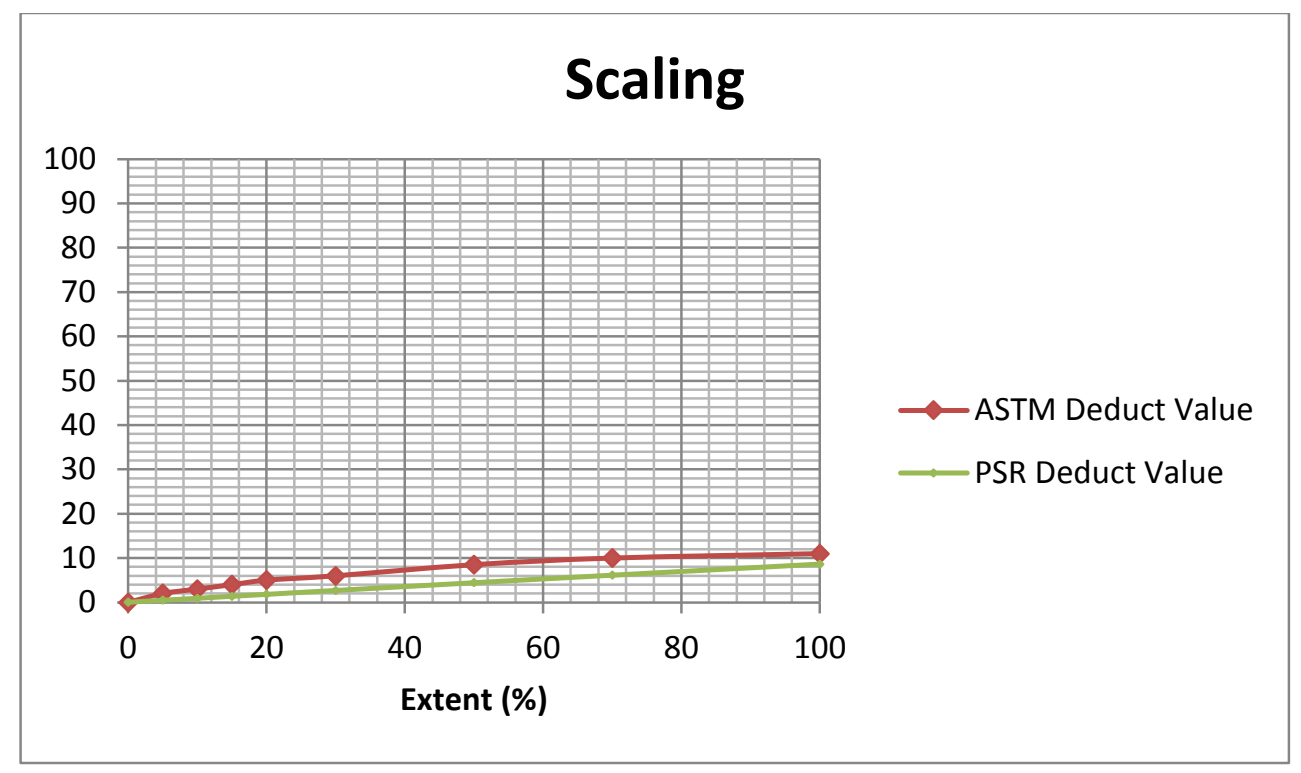

Figure 92: Scaling Deduct Curve Comparison

\section{Patching}

- 0.030

- $\quad$ PSR definition matches PCI Low Severity Patching definition so it was used for comparison. PCI also includes a High Severity Patching which is related to distress found within patching. PSR allows for the recording of distresses within patching so this would be redundant.

- Curve was reduced because well maintained Patching should have a minimal effect on perceived distress. Additionally, distresses within Patches are counted separately.

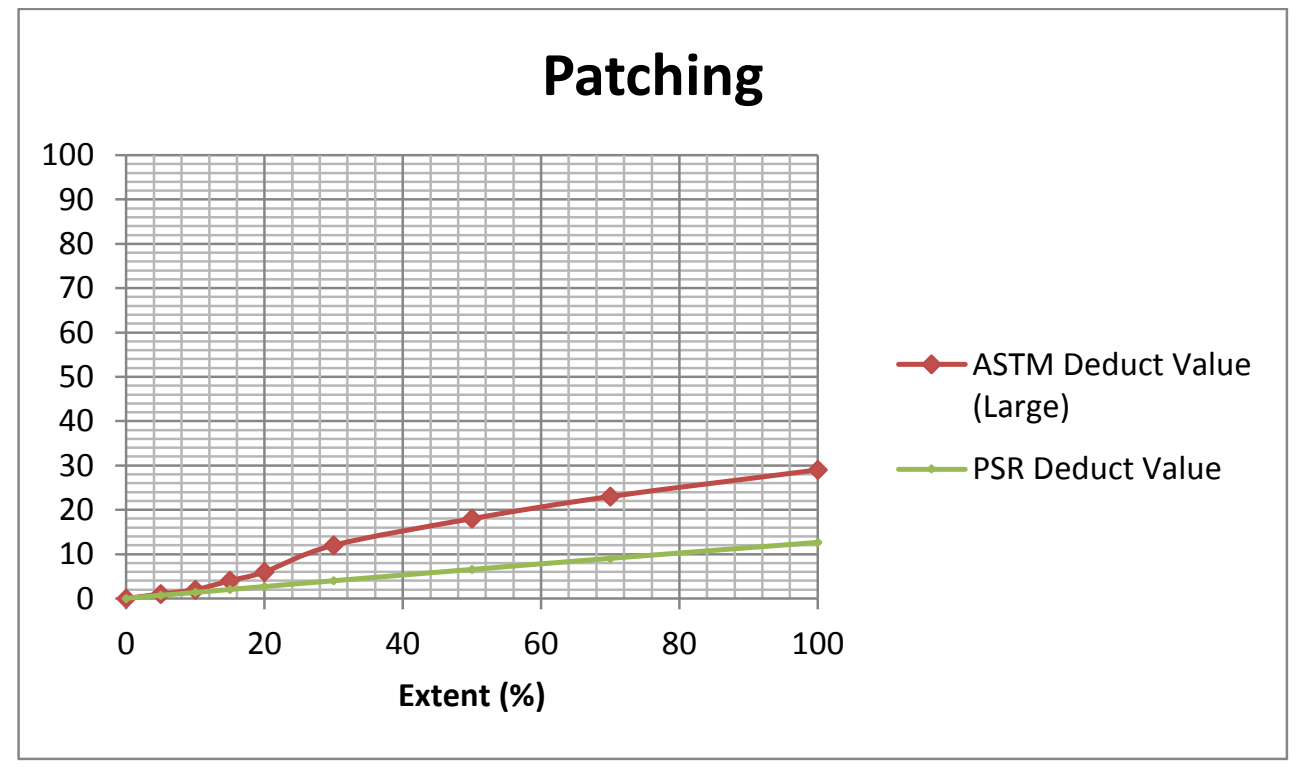

Figure 93: Patching Deduct Curve Comparison 


\section{Popouts}

- 0.035

- Definition matches.

- At the request of the WVDOH, no reduction was made to the curve. This is to account for the potential of a road with no distress other than excessive popouts which would require no correction factor in $\mathrm{PCl}$.

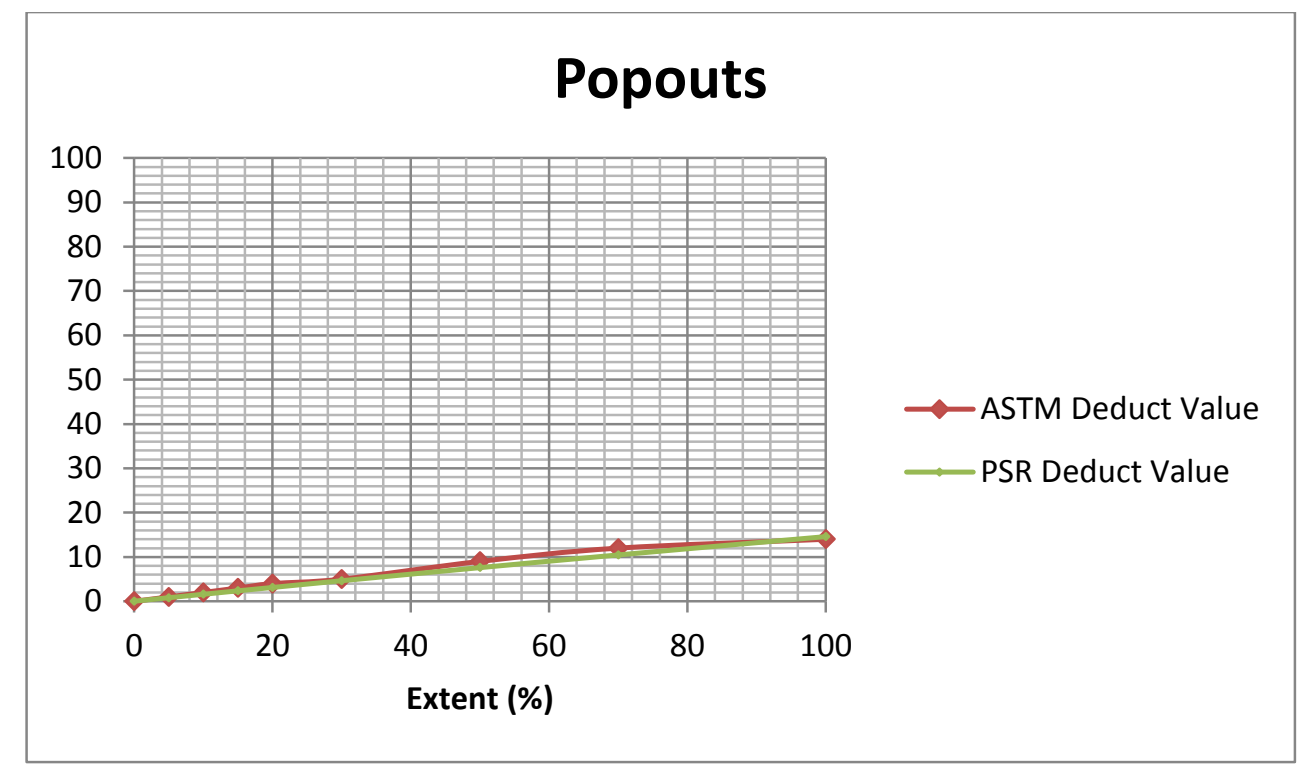

Figure 94: Popouts Deduct Curve Comparison 


\section{Appendix C. FWD text files}

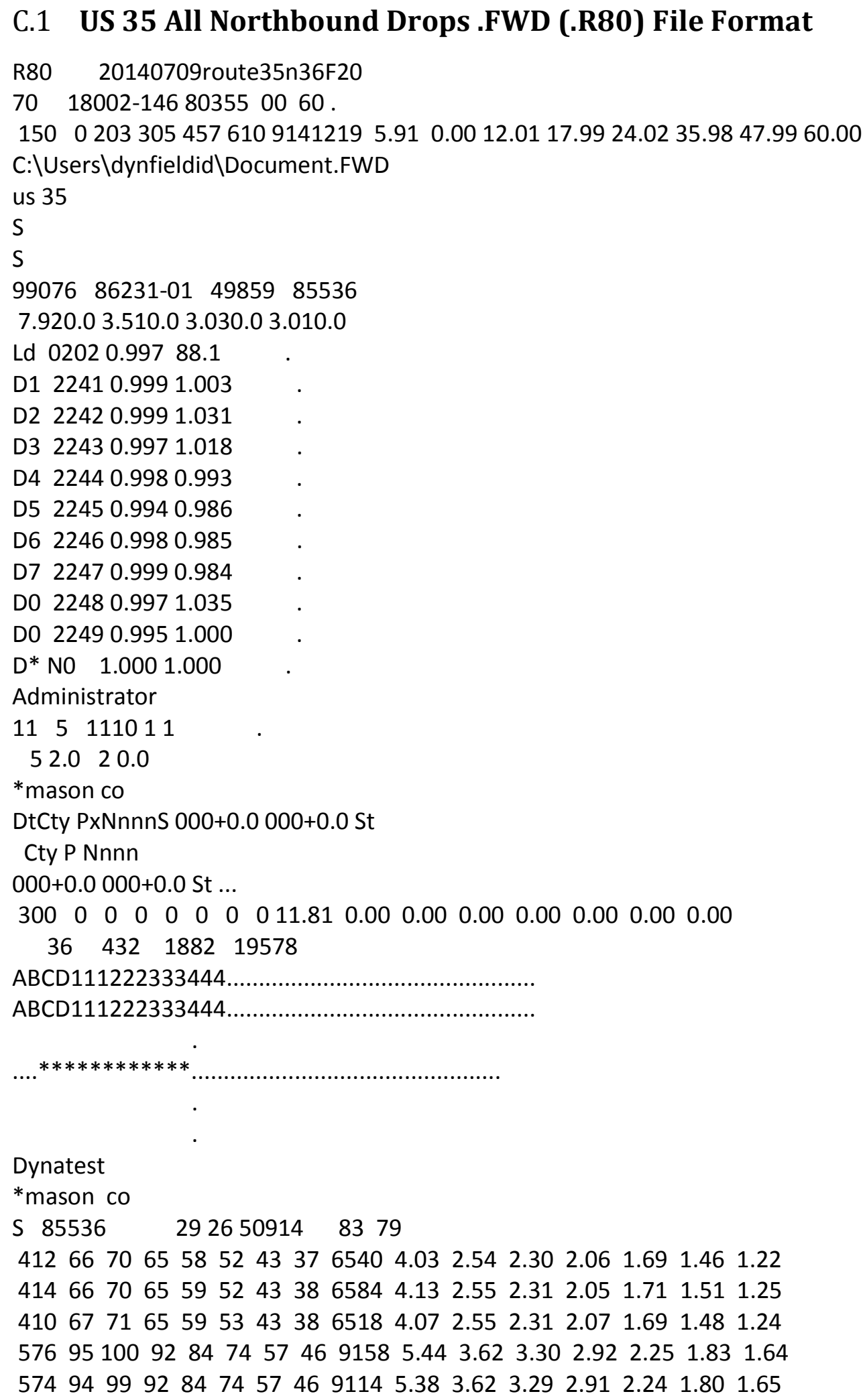




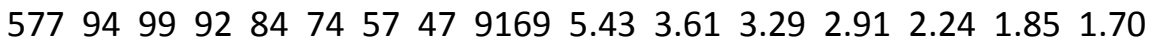
$7871301371281161038060125107.455 .03 \quad 4.564 .043 .152 .371 .83$ $7991311381281171048161126967.48 \quad 5.054 .594 .083 .18 \quad 2.401 .82$ $7921311371271161038060125767.43 \quad 5.004 .564 .06 \quad 3.152 .371 .80$ 1038174182169153137106811649710.006 .656 .045 .394 .193 .172 .31 103317418216815313610680164109.916 .636 .025 .374 .173 .162 .31 $97017418316915313710681154139.906 .656 .04 \quad 5.40 \quad 4.193 .17 \quad 2.33$ S $85013 \quad 292650916 \quad 8478$

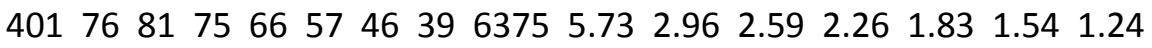
$\begin{array}{llllllllllllllll}402 & 75 & 81 & 74 & 65 & 57 & 45 & 39 & 6386 & 5.35 & 2.93 & 2.56 & 2.24 & 1.78 & 1.54 & 1.20\end{array}$

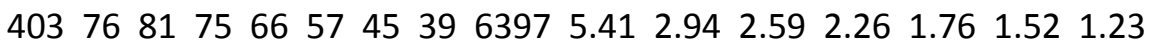

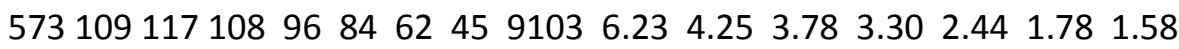

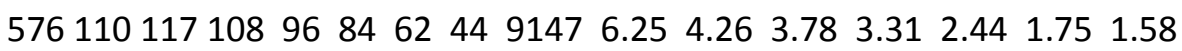

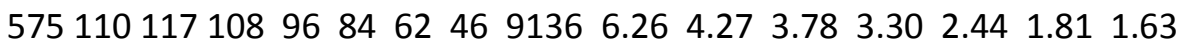
7761521621501341178763123248.625 .905 .264 .613 .432 .491 .82 7841521631501341178863124558.665 .915 .284 .613 .452 .491 .78

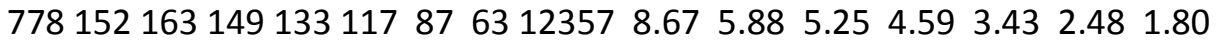
1036205216199178156117851645411.427 .856 .996 .144 .613 .352 .34 $997205216199178156117851584011.397 .856 .996 .14 \quad 4.613 .352 .34$ 983204217200178156118851562111.287 .877 .006 .154 .643 .362 .36 S $83450 \quad 292650920 \quad 8579$ $\begin{array}{llllllllllllllll}399 & 59 & 62 & 56 & 49 & 44 & 41 & 36 & 6343 & 7.87 & 2.20 & 1.93 & 1.75 & 1.63 & 1.43 & 1.05\end{array}$ $\begin{array}{llllllllllllllll}397 & 58 & 61 & 55 & 49 & 44 & 41 & 34 & 6310 & 7.63 & 2.18 & 1.92 & 1.73 & 1.62 & 1.33 & 1.08\end{array}$ $\begin{array}{llllllllllllllll}401 & 60 & 61 & 56 & 48 & 43 & 40 & 36 & 6365 & 8.47 & 2.19 & 1.88 & 1.70 & 1.59 & 1.42 & 1.02\end{array}$

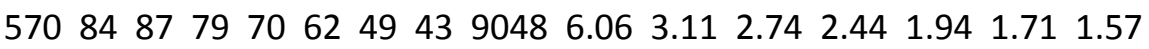
$\begin{array}{llllllllllllllll}573 & 85 & 87 & 79 & 70 & 62 & 49 & 45 & 9103 & 5.80 & 3.11 & 2.74 & 2.43 & 1.94 & 1.78 & 1.54\end{array}$ $\begin{array}{llllllllllllllll}572 & 84 & 87 & 79 & 69 & 61 & 47 & 43 & 9092 & 5.85 & 3.10 & 2.72 & 2.42 & 1.87 & 1.68 & 1.41\end{array}$

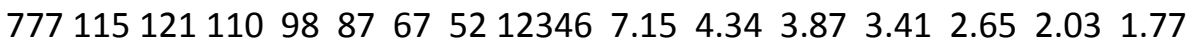

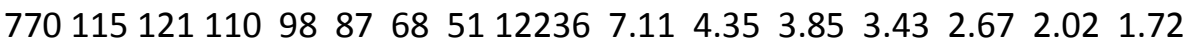
$78111512111098 \quad 876851124117.17 \quad 4.343 .87 \quad 3.442 .662 .02 \quad 1.73$ 102915316114713111691691634410.045 .785 .174 .583 .572 .721 .99 10181531601471311169069161699.915 .775 .154 .573 .562 .711 .99 $10061531611471311179169159829.835 .785 .174 .59 \quad 3.57 \quad 2.701 .99$ S $82928 \quad 292650922 \quad 8580$

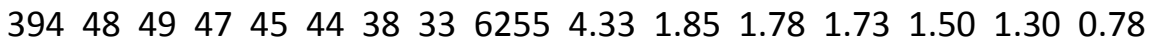
$\begin{array}{llllllllllllllll}399 & 48 & 49 & 47 & 45 & 43 & 38 & 33 & 6343 & 4.20 & 1.84 & 1.76 & 1.70 & 1.48 & 1.28 & 0.74\end{array}$ $\begin{array}{llllllllllllllll}399 & 50 & 49 & 47 & 45 & 44 & 39 & 33 & 6332 & 4.13 & 1.87 & 1.79 & 1.74 & 1.52 & 1.29 & 0.80\end{array}$

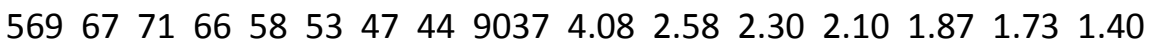
$\begin{array}{llllllllllllllll}570 & 68 & 71 & 66 & 58 & 53 & 47 & 44 & 9059 & 4.01 & 2.60 & 2.30 & 2.07 & 1.87 & 1.72 & 1.43\end{array}$ $\begin{array}{llllllllllllllll}571 & 68 & 71 & 66 & 58 & 52 & 47 & 43 & 9070 & 4.06 & 2.59 & 2.30 & 2.04 & 1.84 & 1.68 & 1.39\end{array}$

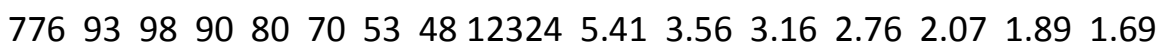

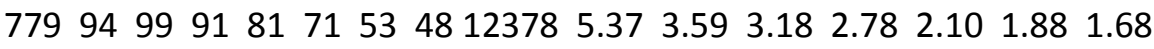

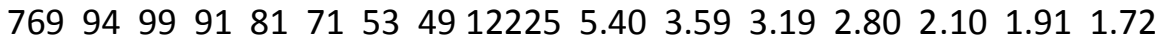
$987127133122108957254156877.42 \quad 4.804 .263 .742 .832 .131 .84$ $978126133122108957254155337.424 .794 .273 .742 .842 .12 \quad 1.83$ $1017126132121108957254161587.414 .784 .26 \quad 3.742 .832 .121 .83$ S $80325 \quad 302750927 \quad 8681$

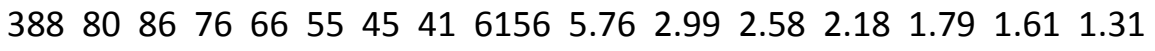


$\begin{array}{llllllllllllllll}390 & 79 & 85 & 75 & 65 & 55 & 44 & 39 & 6189 & 5.77 & 2.97 & 2.56 & 2.17 & 1.74 & 1.54 & 1.22\end{array}$

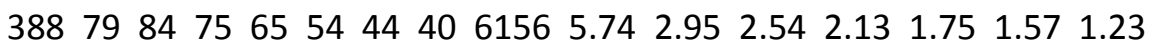

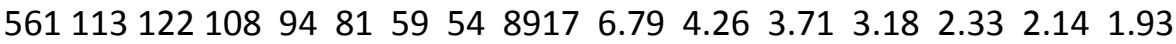

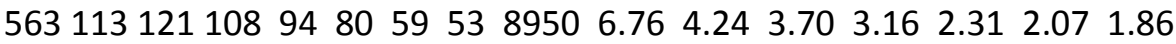

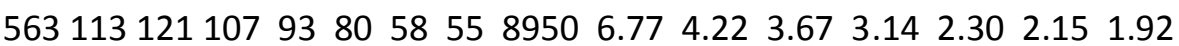
$7721561681501321148462122699.775 .925 .184 .473 .32 \quad 2.452 .32$

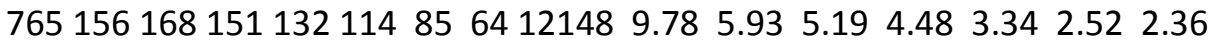
$7721561681501321148562122699.765 .925 .194 .493 .33 \quad 2.432 .30$ $960209224201177153114831524912.727 .926 .966 .03 \quad 4.503 .262 .66$ 984210225202177154115831563212.717 .946 .986 .064 .513 .262 .61 960210225202177154115831524912.697 .946 .986 .064 .523 .262 .62 S $79797 \quad 302750930 \quad 8681$

$\begin{array}{llllllllllllllll}377 & 61 & 68 & 58 & 50 & 46 & 42 & 36 & 5992 & 5.31 & 2.29 & 1.96 & 1.80 & 1.66 & 1.40 & 1.18\end{array}$

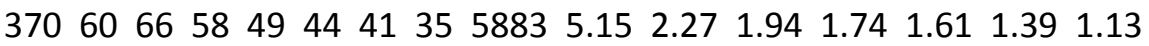

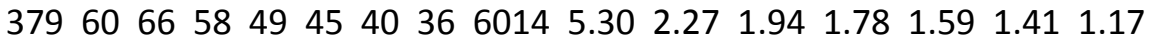
$\begin{array}{llllllllllllllll}550 & 88 & 97 & 85 & 74 & 64 & 56 & 50 & 8731 & 5.93 & 3.35 & 2.90 & 2.50 & 2.22 & 1.98 & 1.71\end{array}$ $\begin{array}{llllllllllllllll}550 & 87 & 97 & 86 & 74 & 64 & 57 & 51 & 8742 & 5.93 & 3.37 & 2.90 & 2.50 & 2.25 & 2.01 & 1.73\end{array}$ $\begin{array}{llllllllllllllll}552 & 87 & 96 & 84 & 73 & 63 & 55 & 49 & 8774 & 5.87 & 3.31 & 2.88 & 2.47 & 2.17 & 1.93 & 1.70\end{array}$

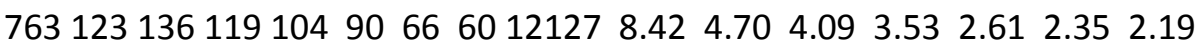

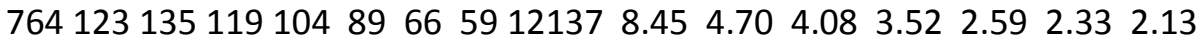

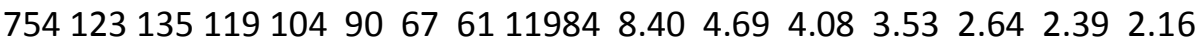
$95916718116114012190691523810.716 .345 .53 \quad 4.78 \quad 3.53 \quad 2.72 \quad 2.53$ $98916818216114012290681570910.736 .35 \quad 5.52 \quad 4.793 .552 .68 \quad 2.52$ $98716818216114112290681568710.696 .355 .54 \quad 4.80 \quad 3.562 .672 .56$ S $79263 \quad 302750933 \quad 8581$

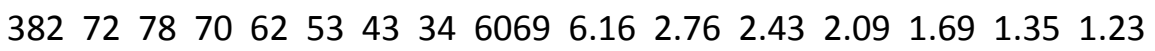
$\begin{array}{llllllllllllllll}383 & 72 & 77 & 69 & 61 & 53 & 43 & 36 & 6091 & 6.39 & 2.73 & 2.39 & 2.08 & 1.68 & 1.43 & 1.27\end{array}$

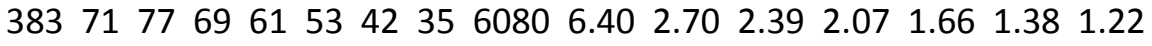

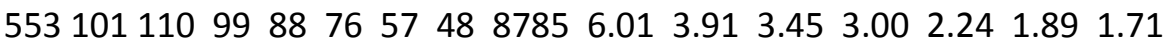

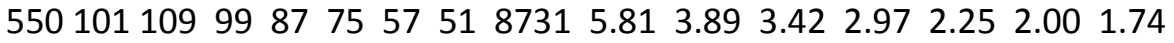

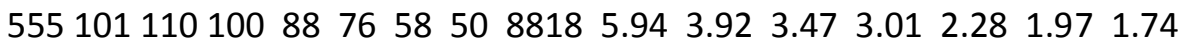
7601371491351191048063120727.815 .304 .694 .103 .142 .492 .09 $7541371501351201058062119847.655 .32 \quad 4.724 .123 .152 .462 .13$ 7591371491351191048062120617.805 .314 .694 .113 .142 .462 .11 $965181196177158138106821532510.106 .986 .215 .43 \quad 4.173 .212 .39$ $981182196177158138106811558810.336 .986 .215 .44 \quad 4.173 .192 .41$ $1000183197178158138106821588410.316 .996 .21 \quad 5.44 \quad 4.18 \quad 3.22 \quad 2.41$ S $78735 \quad 312750935 \quad 8881$

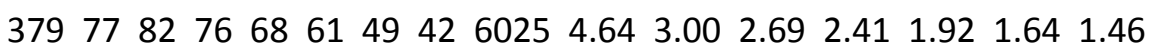

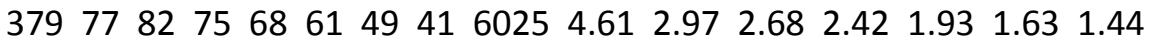

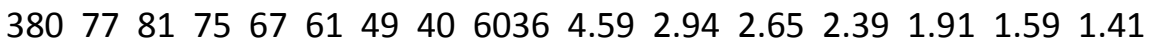

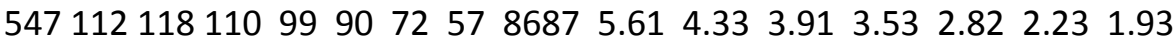

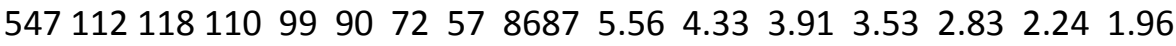

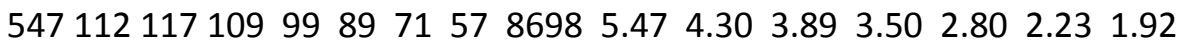
$75615516315313912510180120177.736 .025 .464 .93 \quad 3.973 .152 .46$ $74415516415313912510180118207.75 \quad 6.02 \quad 5.46 \quad 4.943 .98 \quad 3.152 .48$

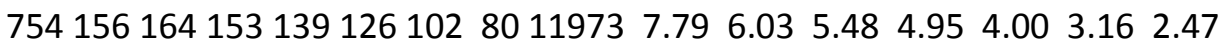
$9922082172031851671351071575210.208 .017 .286 .595 .32 \quad 4.213 .31$ 
$9702082182041851681351071541310.248 .037 .306 .605 .334 .23 \quad 3.32$ $9942082182041861681361081579610.258 .047 .326 .615 .35 \quad 4.243 .31$ S $76074 \quad 302750939 \quad 8681$

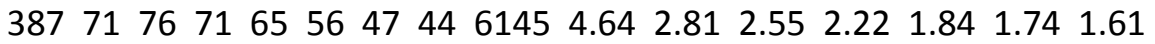
$\begin{array}{llllllllllllllll}393 & 72 & 76 & 72 & 65 & 57 & 46 & 46 & 6244 & 4.49 & 2.82 & 2.55 & 2.24 & 1.82 & 1.81 & 1.65\end{array}$

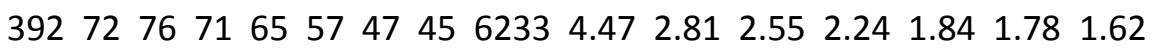

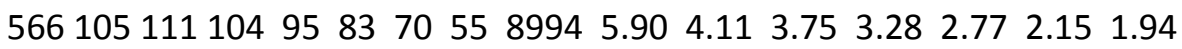

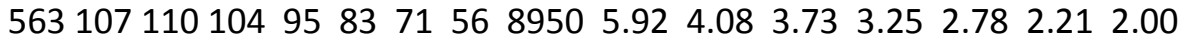

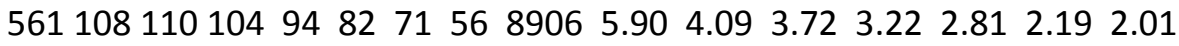
$7611481551461341179977120947.715 .765 .264 .593 .893 .02 \quad 2.31$ $7651471551461331169977121597.675 .735 .24 \quad 4.583 .893 .042 .34$ $7641481561461341189977121377.695 .755 .264 .653 .883 .02 \quad 2.30$ 9982002101971801611341041585110.577 .747 .106 .355 .294 .093 .13 $9812002101971801621341051558810.637 .747 .106 .365 .28 \quad 4.12 \quad 3.14$ 10052012101971811621341051597210.637 .747 .116 .395 .284 .123 .15 S $75552 \quad 302750941 \quad 8681$ $\begin{array}{llllllllllllllll}392 & 53 & 57 & 51 & 47 & 45 & 41 & 36 & 6233 & 4.45 & 2.02 & 1.84 & 1.79 & 1.62 & 1.40 & 1.11\end{array}$ $\begin{array}{llllllllllllllll}392 & 54 & 57 & 51 & 47 & 45 & 41 & 37 & 6233 & 4.19 & 2.01 & 1.85 & 1.79 & 1.61 & 1.44 & 1.15\end{array}$

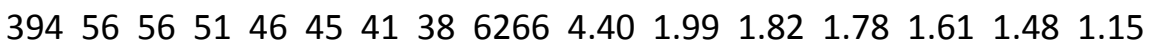
$\begin{array}{llllllllllllllll}565 & 81 & 80 & 72 & 62 & 55 & 51 & 49 & 8983 & 5.50 & 2.85 & 2.46 & 2.16 & 2.00 & 1.91 & 1.56\end{array}$

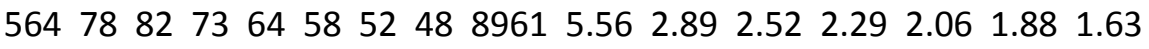

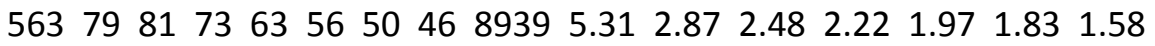

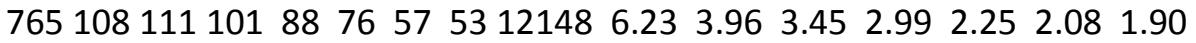

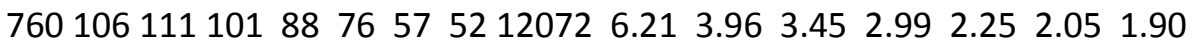

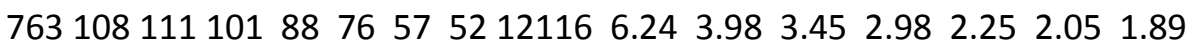
$9911441481341171017656157418.715 .294 .603 .993 .00 \quad 2.212 .10$ 9421441481341171017657149648.675 .284 .593 .983 .012 .242 .10 9801441481341171027657155778.705 .294 .604 .003 .012 .242 .12 S $75024 \quad 322750944 \quad 8981$

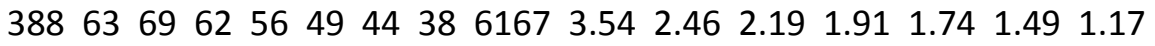
$\begin{array}{llllllllllllllll}392 & 63 & 69 & 63 & 56 & 49 & 44 & 39 & 6233 & 3.57 & 2.48 & 2.19 & 1.91 & 1.73 & 1.52 & 1.19\end{array}$ $\begin{array}{llllllllllllllll}393 & 63 & 69 & 63 & 56 & 49 & 44 & 38 & 6244 & 3.49 & 2.49 & 2.20 & 1.93 & 1.74 & 1.51 & 1.19\end{array}$

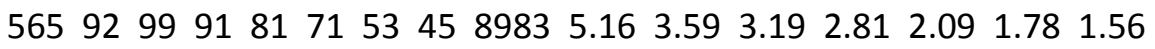
$\begin{array}{llllllllllllllll}565 & 92 & 99 & 91 & 81 & 71 & 53 & 45 & 8972 & 5.17 & 3.58 & 3.19 & 2.81 & 2.10 & 1.76 & 1.55\end{array}$

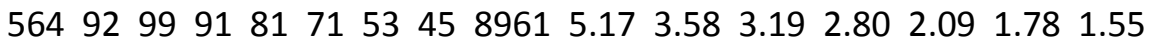

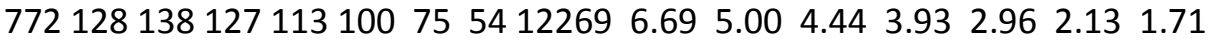
7721291381271131007554122696.655 .004 .443 .932 .962 .131 .68 7691291381271131007554122256.634 .994 .443 .932 .972 .131 .70 $99417318517115313510274157969.096 .726 .02 \quad 5.314 .03 \quad 2.912 .00$ $95817318517115313510274152279.096 .736 .015 .31 \quad 4.03 \quad 2.912 .00$ 93617418517115313510374148769.116 .746 .025 .314 .042 .912 .01 S $74496 \quad 322750946 \quad 8981$

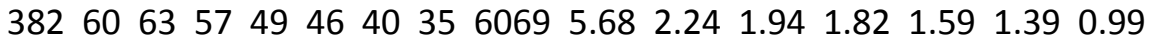
$\begin{array}{llllllllllllllll}388 & 62 & 65 & 58 & 50 & 47 & 41 & 36 & 6167 & 6.65 & 2.29 & 1.98 & 1.84 & 1.61 & 1.43 & 1.02\end{array}$ $\begin{array}{llllllllllllllll}388 & 62 & 65 & 58 & 50 & 47 & 41 & 36 & 6167 & 6.52 & 2.28 & 1.98 & 1.85 & 1.62 & 1.40 & 1.02\end{array}$

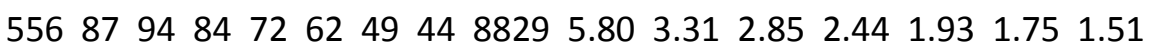

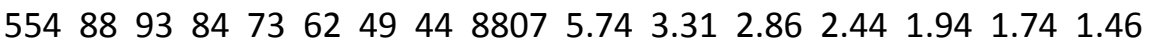
$\begin{array}{llllllllllllllll}558 & 88 & 94 & 85 & 73 & 62 & 50 & 43 & 8862 & 5.62 & 3.33 & 2.87 & 2.44 & 1.95 & 1.71 & 1.45\end{array}$ 
758122130118103876247120397.944 .644 .053 .432 .451 .871 .71

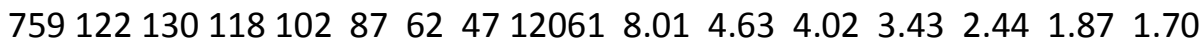

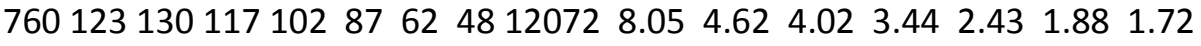
$98516617615913911885581564310.83 \quad 6.275 .494 .66 \quad 3.33 \quad 2.291 .81$ $98716617616013911885581567610.82 \quad 6.28 \quad 5.47 \quad 4.65 \quad 3.34 \quad 2.301 .83$ $100516717615913911885581597210.816 .275 .464 .66 \quad 3.342 .291 .81$ S $73392 \quad 322750953 \quad 9081$ $\begin{array}{llllllllllllllll}382 & 58 & 60 & 55 & 48 & 43 & 38 & 33 & 6069 & 4.54 & 2.15 & 1.89 & 1.71 & 1.48 & 1.30 & 0.83\end{array}$ $\begin{array}{llllllllllllllll}384 & 58 & 61 & 55 & 48 & 43 & 39 & 33 & 6102 & 4.43 & 2.17 & 1.90 & 1.71 & 1.55 & 1.28 & 0.81\end{array}$ $\begin{array}{lllllllllllllllll}383 & 57 & 60 & 55 & 48 & 44 & 39 & 33 & 6080 & 4.38 & 2.16 & 1.89 & 1.74 & 1.55 & 1.31 & 0.85\end{array}$ $\begin{array}{llllllllllllllll}551 & 83 & 88 & 80 & 70 & 60 & 43 & 43 & 8753 & 6.17 & 3.15 & 2.77 & 2.37 & 1.71 & 1.70 & 1.39\end{array}$ $\begin{array}{llllllllllllllll}550 & 83 & 88 & 80 & 71 & 60 & 44 & 43 & 8731 & 6.16 & 3.15 & 2.78 & 2.38 & 1.72 & 1.68 & 1.37\end{array}$

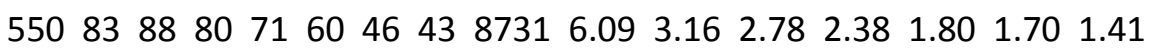
$75211712311399856148119517.93 \quad 4.44 \quad 3.91 \quad 3.362 .411 .881 .67$ $\begin{array}{llllllllllllll}749 & 11612311399 & 85 & 61 & 47 & 11907 & 7.94 & 4.43 & 3.90 & 3.35 & 2.42 & 1.85 & 1.67\end{array}$ $\begin{array}{lllllllllllll}749 & 117123113 & 99 & 85 & 61 & 4611896 & 8.02 & 4.43 & 3.90 & 3.35 & 2.40 & 1.81 & 1.61\end{array}$ $96015916815413511784581526010.626 .055 .33 \quad 4.593 .312 .271 .95$ $94715916815313511784581504010.56 \quad 6.045 .33 \quad 4.593 .31 \quad 2.271 .94$ $98515916815313511784581565410.526 .045 .32 \quad 4.59 \quad 3.30 \quad 2.27 \quad 1.91$ S $72832 \quad 332750956 \quad 9181$ $\begin{array}{llllllllllllllll}376 & 58 & 62 & 56 & 49 & 45 & 40 & 36 & 5981 & 4.26 & 2.21 & 1.94 & 1.76 & 1.57 & 1.40 & 0.97\end{array}$

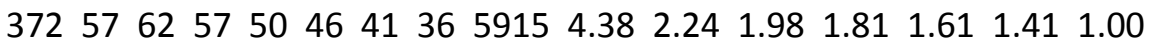
$\begin{array}{llllllllllllllll}381 & 59 & 62 & 56 & 50 & 45 & 39 & 34 & 6047 & 4.38 & 2.22 & 1.96 & 1.76 & 1.53 & 1.35 & 0.94\end{array}$ $\begin{array}{llllllllllllllll}546 & 84 & 89 & 82 & 72 & 62 & 49 & 45 & 8676 & 5.31 & 3.21 & 2.82 & 2.44 & 1.94 & 1.76 & 1.49\end{array}$ $\begin{array}{llllllllllllllll}550 & 83 & 89 & 82 & 72 & 62 & 49 & 45 & 8731 & 5.26 & 3.22 & 2.83 & 2.44 & 1.94 & 1.78 & 1.50\end{array}$ $\begin{array}{llllllllllllllll}548 & 84 & 89 & 82 & 72 & 63 & 50 & 46 & 8709 & 5.24 & 3.22 & 2.83 & 2.47 & 1.96 & 1.81 & 1.53\end{array}$

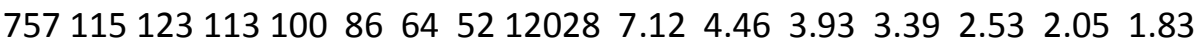
$755115123113100866552119957.084 .453 .943 .392 .542 .03 \quad 1.85$ $758115123113100866452120397.094 .463 .943 .38 \quad 2.532 .061 .84$ $9951541641501321158663158079.705 .915 .214 .53 \quad 3.372 .471 .99$ 9781551641501321158662155339.675 .915 .214 .543 .382 .461 .97 $10021541641501331158663159179.615 .925 .22 \quad 4.543 .382 .47 \quad 1.97$ S $70229 \quad 332751000 \quad 9281$

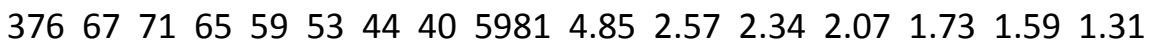

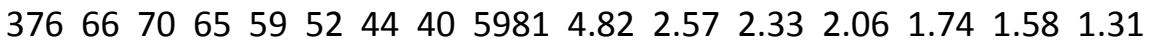
$\begin{array}{llllllllllllllll}376 & 66 & 70 & 65 & 59 & 53 & 42 & 40 & 5981 & 4.80 & 2.57 & 2.33 & 2.07 & 1.67 & 1.56 & 1.30\end{array}$

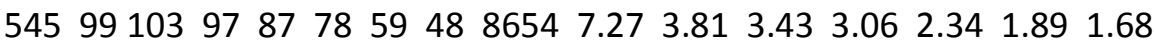
$\begin{array}{llllllllllllllll}545 & 99 & 104 & 97 & 87 & 78 & 60 & 48 & 8654 & 7.22 & 3.82 & 3.44 & 3.06 & 2.35 & 1.89 & 1.67\end{array}$

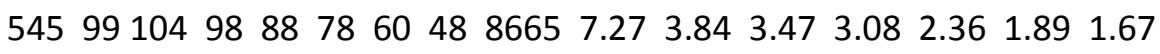
$7521381451361231108564119409.035 .344 .864 .33 \quad 3.342 .511 .98$ 7451381461361241108564118428.955 .364 .874 .343 .342 .512 .02 7481381451361241108564118868.935 .354 .874 .343 .342 .512 .01 907189197185168150117871441612.597 .296 .635 .914 .593 .442 .52 903189198185169150117881433912.467 .306 .645 .924 .603 .452 .53 $932189198185168150116871479912.347 .286 .635 .92 \quad 4.573 .442 .52$ S $69696 \quad 332751003 \quad 9281$

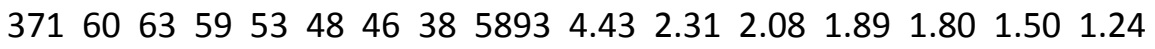
$\begin{array}{llllllllllllllll}373 & 60 & 63 & 59 & 53 & 47 & 44 & 37 & 5926 & 4.31 & 2.31 & 2.08 & 1.86 & 1.74 & 1.47 & 1.20\end{array}$ 


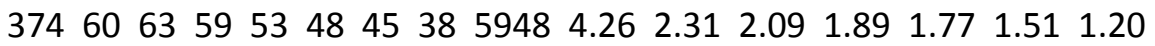
$\begin{array}{llllllllllllllll}549 & 87 & 91 & 85 & 77 & 69 & 57 & 50 & 8720 & 5.60 & 3.36 & 3.04 & 2.73 & 2.26 & 1.98 & 1.81\end{array}$ $\begin{array}{llllllllllllllll}547 & 86 & 91 & 85 & 77 & 69 & 57 & 50 & 8687 & 5.51 & 3.36 & 3.05 & 2.73 & 2.23 & 1.98 & 1.81\end{array}$ $\begin{array}{llllllllllllllll}548 & 87 & 92 & 86 & 78 & 70 & 59 & 53 & 8709 & 5.54 & 3.39 & 3.07 & 2.76 & 2.34 & 2.09 & 1.86\end{array}$

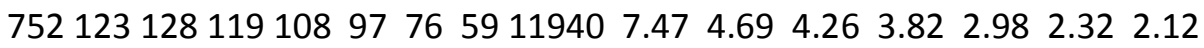

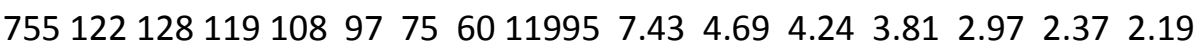
750122127119108977560119187.414 .694 .253 .802 .972 .352 .17 98416317116014513010176156329.966 .305 .705 .103 .973 .012 .50 94416317016014412910176149979.916 .285 .685 .093 .963 .002 .58 98716317016014512910176156879.906 .295 .695 .093 .963 .002 .50 S $67088 \quad 332851008 \quad 9182$

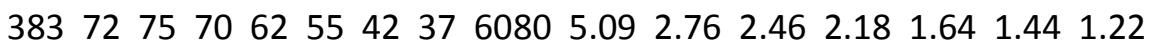
$\begin{array}{llllllllllllllll}384 & 73 & 75 & 70 & 63 & 55 & 43 & 37 & 6102 & 5.10 & 2.76 & 2.47 & 2.17 & 1.68 & 1.46 & 1.28\end{array}$

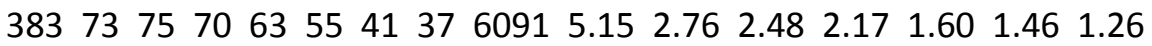
$\begin{array}{llllllllllllll}553103108 & 101 & 91 & 79 & 60 & 46 & 8785 & 5.50 & 3.97 & 3.57 & 3.11 & 2.35 & 1.80 & 1.59\end{array}$

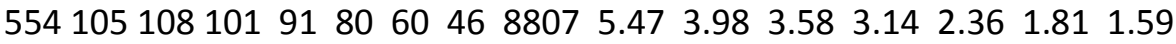

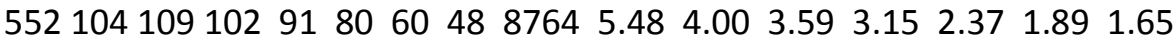

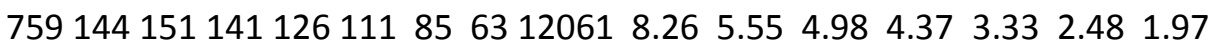
$7491431501401261108462118968.275 .514 .954 .35 \quad 3.30 \quad 2.461 .96$ 7511441501411261108463119298.295 .544 .974 .353 .312 .481 .93 $985193201188169148113841564310.617 .406 .675 .834 .43 \quad 3.312 .43$ $920193201188169148113851461310.597 .406 .675 .83 \quad 4.43 \quad 3.33 \quad 2.43$ $886192201188169148113841407610.587 .406 .66 \quad 5.844 .43 \quad 3.302 .44$ S $66560 \quad 322751011 \quad 9080$

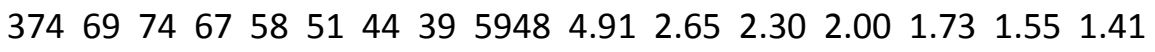

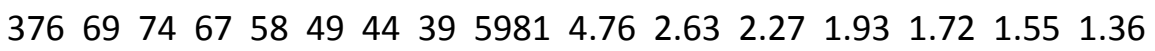

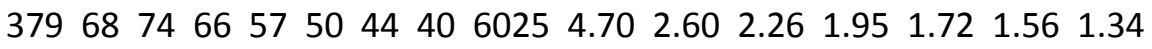

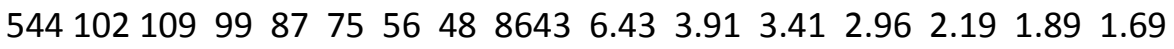

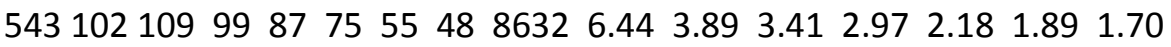
$\begin{array}{lllllllllllllll}545102 & 109 & 99 & 87 & 76 & 55 & 47 & 8654 & 6.43 & 3.89 & 3.41 & 3.00 & 2.18 & 1.87 & 1.74\end{array}$ 7541431551411251098259119738.815 .544 .914 .303 .212 .332 .03

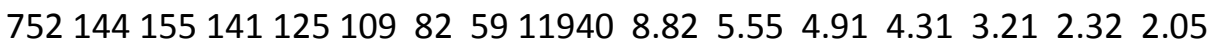
$7471441551411251108259118648.815 .564 .92 \quad 4.33 \quad 3.212 .332 .05$ $969198210191170150112821539112.157 .536 .695 .894 .42 \quad 3.232 .32$ $980198210192170151113821556612.127 .546 .715 .934 .43 \quad 3.242 .35$ $911198210191170150112821447112.047 .536 .695 .914 .42 \quad 3.232 .35$ S $65261 \quad 302651017 \quad 8779$

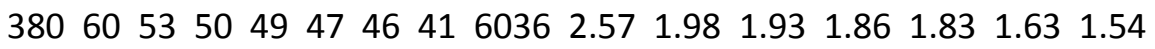

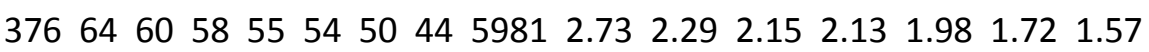
$\begin{array}{llllllllllllllll}378 & 60 & 52 & 50 & 48 & 46 & 44 & 42 & 6003 & 2.56 & 1.96 & 1.88 & 1.80 & 1.75 & 1.64 & 1.49\end{array}$ $\begin{array}{llllllllllllllll}548 & 86 & 75 & 71 & 66 & 60 & 58 & 56 & 8709 & 3.09 & 2.80 & 2.60 & 2.38 & 2.29 & 2.20 & 1.98\end{array}$

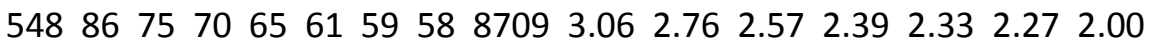
$\begin{array}{llllllllllllllll}545 & 86 & 74 & 69 & 65 & 61 & 56 & 56 & 8665 & 3.13 & 2.73 & 2.54 & 2.42 & 2.21 & 2.20 & 1.92\end{array}$

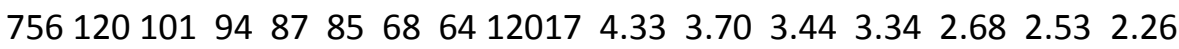

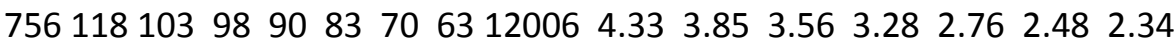

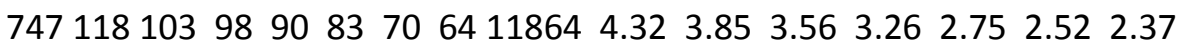
9781611371281191139279155445.825 .024 .684 .443 .633 .102 .60 9491611381301211119378150845.825 .134 .764 .373 .683 .062 .65 
9661611381301211129378153475.835 .124 .754 .393 .673 .072 .65 S $64749 \quad 322751022 \quad 8980$

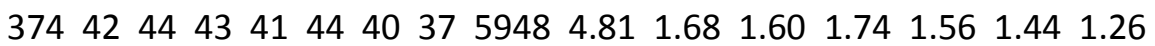
$\begin{array}{llllllllllllllll}376 & 43 & 44 & 43 & 41 & 44 & 40 & 36 & 5981 & 5.20 & 1.68 & 1.63 & 1.72 & 1.56 & 1.43 & 1.21\end{array}$

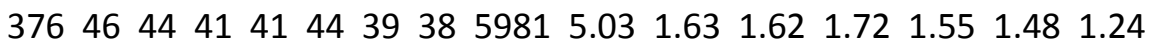
$\begin{array}{llllllllllllllll}543 & 59 & 56 & 55 & 54 & 54 & 51 & 48 & 8621 & 2.18 & 2.17 & 2.12 & 2.14 & 2.02 & 1.88 & 1.74\end{array}$ $\begin{array}{llllllllllllllll}552 & 59 & 58 & 57 & 56 & 54 & 52 & 47 & 8774 & 2.25 & 2.23 & 2.19 & 2.11 & 2.04 & 1.85 & 1.71\end{array}$ $\begin{array}{llllllllllllllll}550 & 59 & 57 & 56 & 54 & 55 & 52 & 49 & 8731 & 2.28 & 2.20 & 2.13 & 2.17 & 2.03 & 1.91 & 1.74\end{array}$

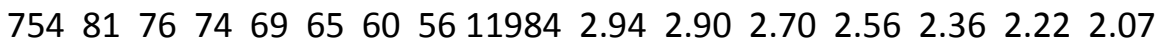
$\begin{array}{llllllllllllllll}751 & 81 & 76 & 74 & 69 & 65 & 60 & 56 & 11929 & 2.89 & 2.90 & 2.71 & 2.54 & 2.36 & 2.20 & 2.05\end{array}$ $\begin{array}{lllllllllllllllll}752 & 81 & 76 & 74 & 69 & 65 & 59 & 56 & 11940 & 2.81 & 2.90 & 2.72 & 2.54 & 2.34 & 2.22 & 2.08\end{array}$

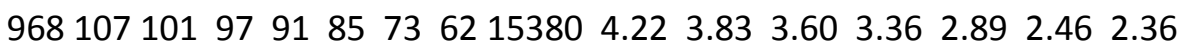

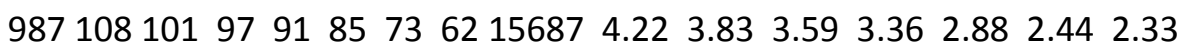

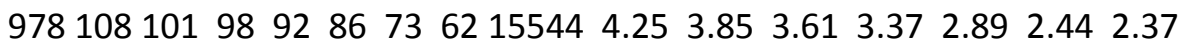
S $62336 \quad 322751029 \quad 9081$ $\begin{array}{llllllllllllllll}368 & 43 & 41 & 43 & 41 & 34 & 31 & 20 & 5839 & 5.52 & 1.71 & 1.61 & 1.32 & 1.22 & 0.80 & 0.97\end{array}$ $\begin{array}{llllllllllllllll}371 & 40 & 40 & 42 & 41 & 33 & 31 & 21 & 5893 & 5.93 & 1.65 & 1.62 & 1.31 & 1.22 & 0.81 & 0.92\end{array}$ $\begin{array}{llllllllllllllll}368 & 40 & 40 & 40 & 40 & 32 & 30 & 20 & 5839 & 6.48 & 1.59 & 1.58 & 1.26 & 1.17 & 0.78 & 0.88\end{array}$ $\begin{array}{llllllllllllllll}539 & 51 & 48 & 49 & 50 & 47 & 44 & 38 & 8555 & 2.06 & 1.91 & 1.96 & 1.84 & 1.74 & 1.51 & 1.32\end{array}$ $\begin{array}{llllllllllllllll}540 & 51 & 48 & 48 & 50 & 46 & 44 & 38 & 8577 & 1.95 & 1.89 & 1.96 & 1.82 & 1.72 & 1.49 & 1.45\end{array}$ $\begin{array}{llllllllllllllll}544 & 52 & 49 & 49 & 51 & 45 & 45 & 38 & 8643 & 1.90 & 1.92 & 2.01 & 1.78 & 1.77 & 1.49 & 1.33\end{array}$ $\begin{array}{llllllllllllllll}740 & 53 & 54 & 51 & 55 & 52 & 52 & 48 & 11754 & 2.73 & 2.02 & 2.15 & 2.06 & 2.03 & 1.89 & 1.51\end{array}$ $\begin{array}{llllllllllllllll}742 & 52 & 53 & 51 & 53 & 52 & 50 & 47 & 11787 & 1.73 & 2.02 & 2.07 & 2.04 & 1.98 & 1.84 & 1.53\end{array}$ $\begin{array}{llllllllllllllll}750 & 52 & 52 & 51 & 53 & 53 & 49 & 47 & 11918 & 1.67 & 2.02 & 2.09 & 2.07 & 1.93 & 1.85 & 1.75\end{array}$

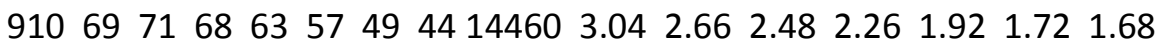

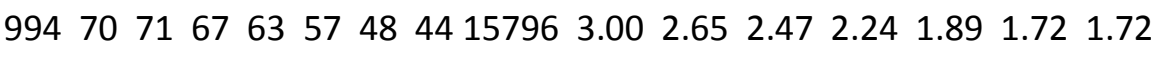

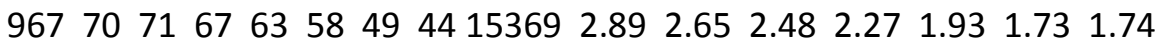
S $61818 \quad 332851033 \quad 9182$ $\begin{array}{llllllllllllllll}368 & 46 & 42 & 45 & 41 & 41 & 39 & 30 & 5839 & 5.92 & 1.78 & 1.63 & 1.63 & 1.52 & 1.18 & 0.90\end{array}$ $\begin{array}{llllllllllllllll}367 & 47 & 40 & 43 & 41 & 40 & 37 & 28 & 5828 & 7.06 & 1.69 & 1.63 & 1.58 & 1.47 & 1.10 & 0.77\end{array}$

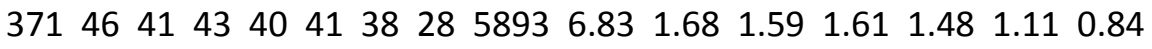
$\begin{array}{llllllllllllllll}533 & 55 & 50 & 49 & 48 & 52 & 50 & 41 & 8468 & 2.38 & 1.92 & 1.90 & 2.06 & 1.96 & 1.63 & 1.40\end{array}$ $\begin{array}{llllllllllllllll}533 & 55 & 50 & 50 & 48 & 52 & 51 & 40 & 8468 & 2.37 & 1.95 & 1.89 & 2.03 & 2.00 & 1.57 & 1.46\end{array}$

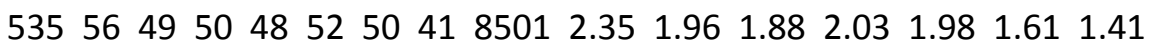
$\begin{array}{llllllllllllllll}740 & 59 & 59 & 56 & 54 & 54 & 54 & 48 & 11754 & 1.87 & 2.20 & 2.11 & 2.13 & 2.11 & 1.89 & 1.72\end{array}$ $\begin{array}{llllllllllllllll}729 & 58 & 58 & 55 & 52 & 54 & 52 & 45 & 11579 & 1.89 & 2.17 & 2.06 & 2.11 & 2.03 & 1.79 & 1.84\end{array}$ $\begin{array}{llllllllllllllll}728 & 59 & 59 & 55 & 53 & 54 & 53 & 46 & 11568 & 2.06 & 2.18 & 2.07 & 2.13 & 2.09 & 1.83 & 1.86\end{array}$ $\begin{array}{llllllllllllllll}913 & 79 & 80 & 76 & 72 & 68 & 57 & 48 & 14504 & 3.16 & 3.00 & 2.82 & 2.66 & 2.23 & 1.89 & 1.83\end{array}$

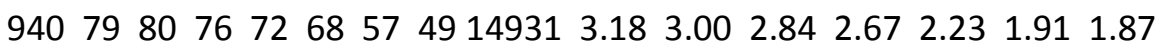

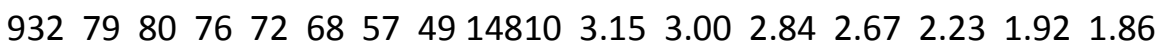
S $60783 \quad 362851039 \quad 9683$

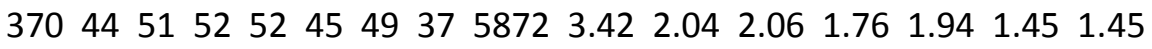

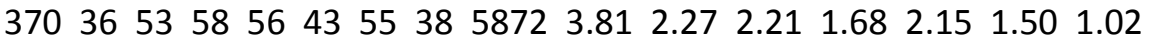
$\begin{array}{llllllllllllllll}370 & 38 & 49 & 53 & 52 & 44 & 49 & 39 & 5872 & 3.66 & 2.07 & 2.05 & 1.72 & 1.94 & 1.54 & 1.43\end{array}$ $\begin{array}{llllllllllllllll}529 & 61 & 61 & 59 & 57 & 58 & 55 & 49 & 8402 & 2.33 & 2.33 & 2.26 & 2.27 & 2.16 & 1.94 & 2.03\end{array}$ $\begin{array}{llllllllllllllll}530 & 60 & 61 & 60 & 58 & 57 & 54 & 51 & 8424 & 2.39 & 2.35 & 2.29 & 2.23 & 2.13 & 2.02 & 2.17\end{array}$ $\begin{array}{llllllllllllllll}527 & 61 & 60 & 59 & 58 & 53 & 56 & 48 & 8380 & 2.36 & 2.31 & 2.29 & 2.09 & 2.22 & 1.90 & 1.81\end{array}$ $\begin{array}{llllllllllllllll}716 & 88 & 83 & 81 & 77 & 75 & 65 & 56 & 11371 & 3.52 & 3.20 & 3.05 & 2.97 & 2.57 & 2.19 & 2.06\end{array}$ 
$\begin{array}{llllllllllllllll}725 & 87 & 84 & 82 & 77 & 75 & 66 & 55 & 11513 & 3.35 & 3.22 & 3.05 & 2.96 & 2.58 & 2.18 & 1.93\end{array}$ $\begin{array}{llllllllllllllll}709 & 87 & 84 & 82 & 77 & 75 & 66 & 55 & 11261 & 3.36 & 3.21 & 3.05 & 2.97 & 2.58 & 2.16 & 1.88\end{array}$ $9671131131091041008675153584.574 .294 .093 .93 \quad 3.392 .942 .46$ $9581141131101051008675152164.584 .324 .12 \quad 3.95 \quad 3.392 .952 .47$ 9051141131101041008675143834.564 .334 .103 .943 .382 .942 .47 S $60276 \quad 372851041 \quad 9882$

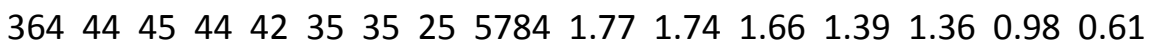
$\begin{array}{llllllllllllllll}361 & 43 & 49 & 45 & 44 & 37 & 38 & 23 & 5740 & 1.56 & 1.79 & 1.72 & 1.46 & 1.51 & 0.91 & 0.60\end{array}$ $\begin{array}{llllllllllllllll}356 & 39 & 40 & 40 & 38 & 36 & 34 & 25 & 5652 & 2.00 & 1.56 & 1.50 & 1.43 & 1.34 & 0.98 & 0.58\end{array}$ $\begin{array}{llllllllllllllll}533 & 47 & 61 & 55 & 53 & 50 & 50 & 40 & 8468 & 2.49 & 2.15 & 2.09 & 1.96 & 1.98 & 1.57 & 1.27\end{array}$ $\begin{array}{llllllllllllllll}535 & 48 & 57 & 53 & 52 & 48 & 45 & 38 & 8501 & 2.26 & 2.09 & 2.06 & 1.88 & 1.79 & 1.49 & 1.15\end{array}$ $\begin{array}{llllllllllllllll}536 & 46 & 60 & 56 & 54 & 51 & 52 & 40 & 8512 & 2.13 & 2.19 & 2.12 & 1.99 & 2.03 & 1.57 & 1.24\end{array}$ $\begin{array}{llllllllllllllll}734 & 58 & 60 & 58 & 55 & 52 & 53 & 46 & 11666 & 2.46 & 2.28 & 2.15 & 2.06 & 2.08 & 1.82 & 1.59\end{array}$

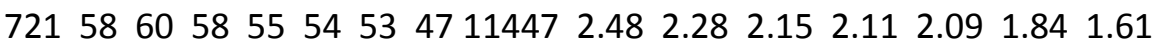
$\begin{array}{llllllllllllllll}727 & 58 & 60 & 58 & 55 & 53 & 53 & 46 & 11557 & 2.50 & 2.28 & 2.15 & 2.10 & 2.07 & 1.83 & 1.63\end{array}$ $\begin{array}{llllllllllllllll}978 & 80 & 81 & 78 & 74 & 69 & 60 & 51 & 15533 & 3.52 & 3.09 & 2.93 & 2.72 & 2.36 & 2.00 & 1.75\end{array}$ $\begin{array}{llllllllllllllll}949 & 80 & 82 & 79 & 75 & 70 & 61 & 51 & 15073 & 3.49 & 3.11 & 2.94 & 2.76 & 2.39 & 1.99 & 1.80\end{array}$

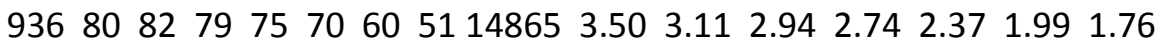
S $57705 \quad 342951047 \quad 9384$

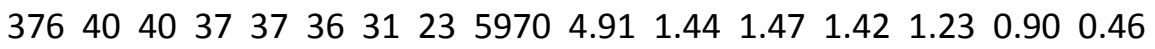
$\begin{array}{llllllllllllllll}377 & 44 & 44 & 42 & 40 & 40 & 34 & 27 & 5992 & 5.42 & 1.67 & 1.57 & 1.56 & 1.35 & 1.07 & 0.63\end{array}$ $\begin{array}{llllllllllllllll}381 & 44 & 44 & 41 & 40 & 40 & 35 & 27 & 6047 & 5.32 & 1.61 & 1.59 & 1.57 & 1.39 & 1.07 & 0.57\end{array}$ $\begin{array}{llllllllllllllll}550 & 52 & 53 & 49 & 51 & 51 & 50 & 47 & 8742 & 2.71 & 1.92 & 2.00 & 2.00 & 1.95 & 1.84 & 1.66\end{array}$ $\begin{array}{llllllllllllllll}553 & 53 & 53 & 50 & 51 & 51 & 50 & 46 & 8785 & 2.56 & 1.95 & 2.01 & 2.01 & 1.96 & 1.83 & 1.69\end{array}$ $\begin{array}{llllllllllllllll}552 & 52 & 53 & 50 & 51 & 51 & 49 & 44 & 8774 & 2.65 & 1.95 & 1.99 & 1.99 & 1.92 & 1.74 & 1.58\end{array}$

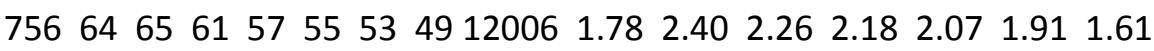
$\begin{array}{llllllllllllllll}755 & 63 & 65 & 61 & 57 & 54 & 52 & 46 & 11995 & 1.79 & 2.39 & 2.25 & 2.13 & 2.04 & 1.83 & 1.58\end{array}$ $\begin{array}{llllllllllllllll}749 & 63 & 64 & 61 & 57 & 55 & 52 & 48 & 11896 & 1.80 & 2.39 & 2.25 & 2.15 & 2.06 & 1.90 & 1.70\end{array}$ $\begin{array}{llllllllllllllll}978 & 86 & 85 & 81 & 76 & 70 & 59 & 53 & 15533 & 3.91 & 3.20 & 2.98 & 2.77 & 2.34 & 2.07 & 2.01\end{array}$ $\begin{array}{llllllllllllllll}993 & 86 & 85 & 82 & 76 & 71 & 59 & 52 & 15774 & 3.94 & 3.21 & 2.99 & 2.78 & 2.34 & 2.05 & 1.99\end{array}$ $\begin{array}{llllllllllllllll}995 & 85 & 85 & 81 & 76 & 71 & 60 & 53 & 15807 & 3.93 & 3.20 & 2.99 & 2.78 & 2.35 & 2.07 & 1.97\end{array}$ S $57182 \quad 342951048 \quad 9383$ $\begin{array}{llllllllllllllll}375 & 48 & 46 & 46 & 45 & 44 & 40 & 39 & 5959 & 1.88 & 1.83 & 1.78 & 1.74 & 1.57 & 1.53 & 1.22\end{array}$ $\begin{array}{llllllllllllllll}370 & 47 & 45 & 45 & 44 & 43 & 41 & 39 & 5883 & 1.98 & 1.76 & 1.73 & 1.70 & 1.62 & 1.52 & 1.16\end{array}$ $\begin{array}{llllllllllllllll}372 & 48 & 44 & 45 & 45 & 44 & 40 & 38 & 5904 & 1.98 & 1.78 & 1.76 & 1.74 & 1.58 & 1.50 & 1.17\end{array}$ $\begin{array}{llllllllllllllll}541 & 54 & 52 & 51 & 51 & 51 & 48 & 49 & 8588 & 3.26 & 2.00 & 2.02 & 2.02 & 1.89 & 1.93 & 1.64\end{array}$

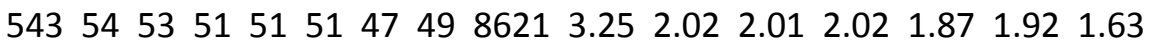

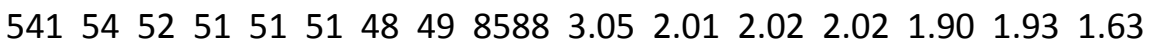

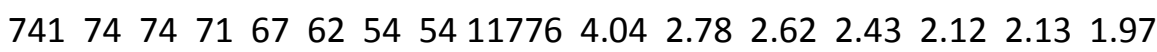
$\begin{array}{llllllllllllllll}741 & 73 & 73 & 71 & 66 & 61 & 55 & 54 & 11765 & 3.89 & 2.78 & 2.60 & 2.42 & 2.15 & 2.11 & 1.96\end{array}$

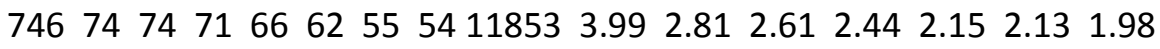

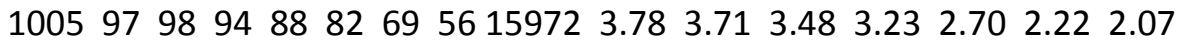
$\begin{array}{llllllllllllllll}928 & 97 & 98 & 94 & 88 & 82 & 68 & 57 & 14745 & 4.83 & 3.71 & 3.46 & 3.23 & 2.69 & 2.24 & 2.07\end{array}$

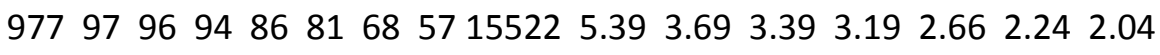
S $55593 \quad 352951052 \quad 9584$

$\begin{array}{llllllllllllllll}381 & 53 & 53 & 52 & 50 & 47 & 45 & 42 & 6047 & 2.22 & 2.04 & 1.96 & 1.85 & 1.78 & 1.67 & 1.50\end{array}$ $\begin{array}{llllllllllllllll}381 & 53 & 54 & 52 & 50 & 47 & 44 & 42 & 6058 & 2.39 & 2.04 & 1.97 & 1.87 & 1.75 & 1.67 & 1.51\end{array}$ $\begin{array}{llllllllllllllll}381 & 53 & 54 & 53 & 50 & 48 & 46 & 43 & 6058 & 2.19 & 2.08 & 1.98 & 1.90 & 1.80 & 1.70 & 1.50\end{array}$ 
$\begin{array}{llllllllllllllll}528 & 73 & 74 & 72 & 69 & 65 & 58 & 53 & 8391 & 3.69 & 2.84 & 2.73 & 2.57 & 2.30 & 2.07 & 1.94\end{array}$

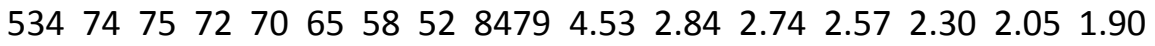

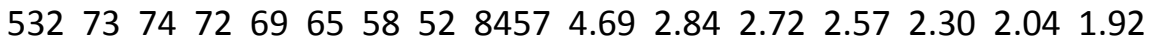

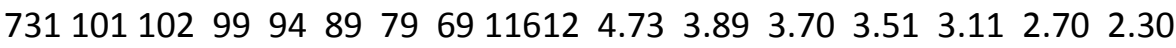

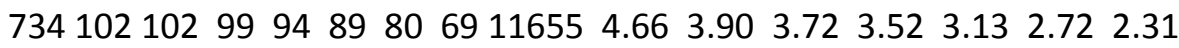

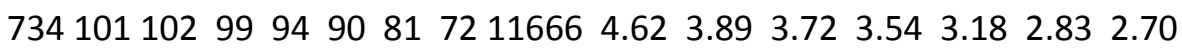
92413513413112511810490146795.325 .164 .914 .634 .093 .543 .00 90113613513112511810490143175.375 .174 .924 .644 .113 .563 .02 $98313513513112411810490156215.385 .154 .904 .634 .08 \quad 3.553 .01$ S $55076 \quad 342951055 \quad 9484$ $\begin{array}{llllllllllllllll}375 & 44 & 45 & 45 & 45 & 37 & 37 & 33 & 5959 & 1.60 & 1.77 & 1.76 & 1.44 & 1.46 & 1.28 & 0.69\end{array}$

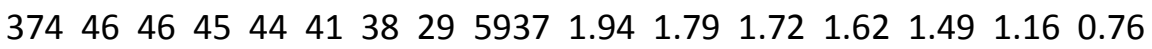
$\begin{array}{llllllllllllllll}371 & 44 & 43 & 46 & 44 & 41 & 40 & 29 & 5893 & 1.69 & 1.82 & 1.74 & 1.62 & 1.56 & 1.16 & 0.72\end{array}$ $\begin{array}{llllllllllllllll}549 & 51 & 50 & 46 & 48 & 46 & 44 & 41 & 8720 & 1.85 & 1.83 & 1.88 & 1.83 & 1.72 & 1.61 & 1.30\end{array}$ $\begin{array}{llllllllllllllll}549 & 51 & 49 & 47 & 49 & 46 & 44 & 41 & 8720 & 1.88 & 1.85 & 1.91 & 1.82 & 1.74 & 1.61 & 1.28\end{array}$ $\begin{array}{llllllllllllllll}550 & 51 & 49 & 46 & 48 & 46 & 44 & 40 & 8742 & 1.84 & 1.81 & 1.89 & 1.81 & 1.73 & 1.57 & 1.26\end{array}$ $\begin{array}{llllllllllllllll}758 & 61 & 62 & 58 & 54 & 51 & 50 & 47 & 12050 & 2.33 & 2.30 & 2.12 & 2.02 & 1.96 & 1.84 & 1.64\end{array}$ $\begin{array}{llllllllllllllll}753 & 61 & 62 & 58 & 54 & 52 & 50 & 48 & 11962 & 2.33 & 2.30 & 2.12 & 2.05 & 1.96 & 1.89 & 1.66\end{array}$ $\begin{array}{llllllllllllllll}756 & 62 & 61 & 58 & 53 & 52 & 48 & 49 & 12017 & 2.35 & 2.29 & 2.10 & 2.06 & 1.90 & 1.92 & 1.61\end{array}$ $\begin{array}{llllllllllllllll}952 & 84 & 84 & 81 & 74 & 68 & 56 & 49 & 15128 & 3.50 & 3.17 & 2.93 & 2.69 & 2.20 & 1.93 & 1.78\end{array}$ $\begin{array}{llllllllllllllll}971 & 85 & 84 & 81 & 74 & 68 & 56 & 48 & 15424 & 3.49 & 3.18 & 2.92 & 2.69 & 2.21 & 1.89 & 1.81\end{array}$

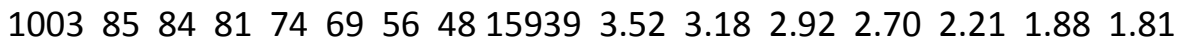
S $50382 \quad 372851101 \quad 9983$

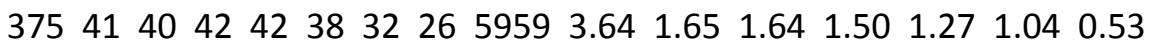
$\begin{array}{llllllllllllllll}373 & 41 & 40 & 42 & 40 & 37 & 33 & 26 & 5926 & 3.67 & 1.65 & 1.59 & 1.47 & 1.30 & 1.04 & 0.52\end{array}$ $\begin{array}{llllllllllllllll}376 & 43 & 41 & 43 & 41 & 38 & 34 & 27 & 5970 & 3.70 & 1.69 & 1.63 & 1.50 & 1.35 & 1.07 & 0.55\end{array}$

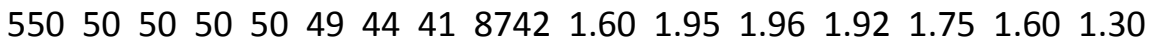

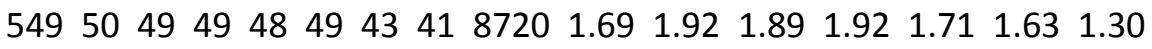
$\begin{array}{llllllllllllllll}548 & 51 & 49 & 49 & 49 & 48 & 44 & 40 & 8709 & 1.61 & 1.93 & 1.91 & 1.90 & 1.72 & 1.56 & 1.27\end{array}$ $\begin{array}{llllllllllllllll}752 & 59 & 59 & 57 & 53 & 51 & 48 & 45 & 11940 & 2.33 & 2.25 & 2.10 & 1.99 & 1.89 & 1.78 & 1.65\end{array}$ $\begin{array}{llllllllllllllll}749 & 59 & 60 & 57 & 53 & 50 & 49 & 46 & 11907 & 2.31 & 2.25 & 2.09 & 1.96 & 1.92 & 1.80 & 1.61\end{array}$ $\begin{array}{llllllllllllllll}750 & 59 & 60 & 57 & 53 & 51 & 47 & 46 & 11918 & 2.31 & 2.26 & 2.10 & 2.00 & 1.87 & 1.83 & 1.65\end{array}$ $\begin{array}{llllllllllllllll}975 & 84 & 83 & 80 & 74 & 68 & 56 & 47 & 15490 & 3.46 & 3.14 & 2.92 & 2.67 & 2.22 & 1.85 & 1.67\end{array}$ $\begin{array}{llllllllllllllll}992 & 84 & 83 & 80 & 75 & 69 & 57 & 46 & 15763 & 3.41 & 3.15 & 2.94 & 2.70 & 2.25 & 1.80 & 1.64\end{array}$ $\begin{array}{llllllllllllllll}953 & 85 & 84 & 80 & 75 & 69 & 58 & 46 & 15139 & 3.42 & 3.16 & 2.95 & 2.71 & 2.27 & 1.81 & 1.69\end{array}$ S $49859 \quad 372851106 \quad 9883$

$\begin{array}{llllllllllllllll}379 & 47 & 44 & 46 & 46 & 46 & 41 & 40 & 6014 & 2.56 & 1.80 & 1.81 & 1.81 & 1.62 & 1.57 & 1.41\end{array}$

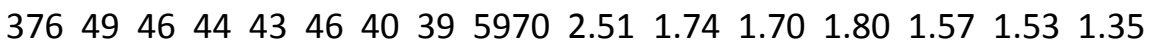

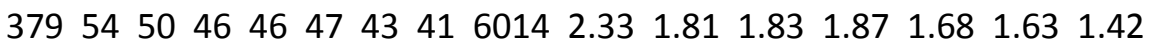
$\begin{array}{llllllllllllllll}543 & 63 & 65 & 64 & 61 & 57 & 51 & 49 & 8632 & 2.34 & 2.52 & 2.39 & 2.26 & 2.02 & 1.93 & 1.81\end{array}$ $\begin{array}{llllllllllllllll}542 & 64 & 65 & 64 & 61 & 57 & 50 & 49 & 8610 & 2.42 & 2.52 & 2.39 & 2.26 & 1.98 & 1.93 & 1.85\end{array}$ $\begin{array}{lllllllllllllllll}543 & 63 & 65 & 64 & 60 & 57 & 51 & 49 & 8632 & 2.39 & 2.52 & 2.38 & 2.24 & 2.01 & 1.91 & 1.81\end{array}$ $\begin{array}{llllllllllllllll}743 & 87 & 88 & 86 & 82 & 77 & 68 & 59 & 11798 & 4.00 & 3.37 & 3.22 & 3.03 & 2.69 & 2.34 & 2.30\end{array}$ $\begin{array}{llllllllllllllll}735 & 87 & 88 & 86 & 82 & 77 & 68 & 60 & 11677 & 4.02 & 3.40 & 3.22 & 3.03 & 2.67 & 2.37 & 2.36\end{array}$ $\begin{array}{llllllllllllllll}738 & 88 & 89 & 87 & 83 & 78 & 70 & 64 & 11721 & 4.09 & 3.44 & 3.28 & 3.09 & 2.76 & 2.50 & 2.46\end{array}$ 9481151161141081029178150624.504 .474 .244 .013 .573 .082 .61 9831171171151081039280156104.584 .514 .274 .063 .613 .152 .70 9771161171141081029178155224.594 .504 .264 .023 .593 .082 .61 
S $48576 \quad 402951120 \quad 10484$

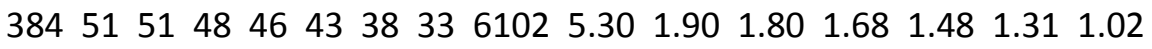
$\begin{array}{llllllllllllllll}381 & 48 & 47 & 45 & 44 & 43 & 39 & 30 & 6058 & 5.54 & 1.79 & 1.72 & 1.71 & 1.55 & 1.19 & 0.93\end{array}$ $\begin{array}{llllllllllllllll}381 & 49 & 48 & 46 & 45 & 45 & 39 & 31 & 6047 & 5.12 & 1.80 & 1.76 & 1.77 & 1.55 & 1.21 & 0.87\end{array}$

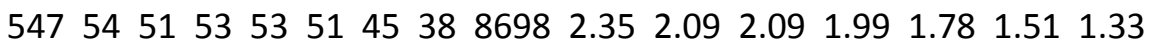

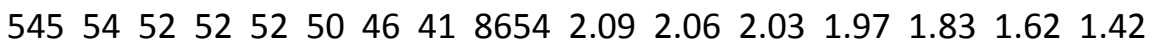

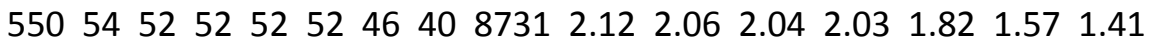
$\begin{array}{llllllllllllllll}754 & 71 & 68 & 66 & 60 & 56 & 53 & 50 & 11973 & 2.65 & 2.59 & 2.36 & 2.19 & 2.08 & 1.97 & 1.74\end{array}$ $\begin{array}{llllllllllllllll}758 & 72 & 68 & 65 & 60 & 54 & 52 & 49 & 12039 & 2.75 & 2.57 & 2.36 & 2.13 & 2.04 & 1.92 & 1.72\end{array}$ $\begin{array}{llllllllllllllll}747 & 71 & 68 & 65 & 59 & 54 & 50 & 49 & 11864 & 2.78 & 2.56 & 2.34 & 2.12 & 1.98 & 1.94 & 1.69\end{array}$

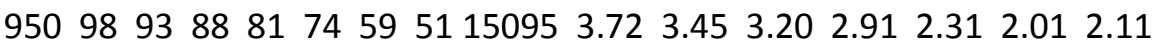
$\begin{array}{llllllllllllllll}942 & 98 & 92 & 88 & 81 & 74 & 59 & 51 & 14964 & 3.74 & 3.47 & 3.19 & 2.91 & 2.32 & 2.02 & 1.89\end{array}$

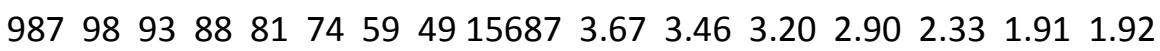
S $48048 \quad 392951124 \quad 10184$

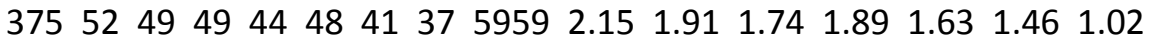
$\begin{array}{llllllllllllllll}370 & 53 & 47 & 49 & 47 & 48 & 41 & 37 & 5872 & 2.45 & 1.92 & 1.84 & 1.88 & 1.63 & 1.47 & 1.06\end{array}$ $\begin{array}{llllllllllllllll}376 & 52 & 48 & 48 & 41 & 46 & 42 & 36 & 5981 & 2.32 & 1.89 & 1.63 & 1.83 & 1.67 & 1.41 & 1.02\end{array}$

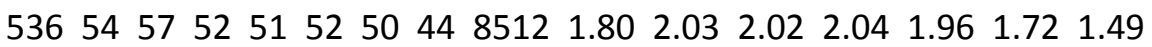
$\begin{array}{llllllllllllllll}539 & 55 & 56 & 53 & 49 & 52 & 52 & 46 & 8566 & 1.77 & 2.10 & 1.94 & 2.05 & 2.05 & 1.81 & 1.44\end{array}$ $\begin{array}{llllllllllllllll}541 & 56 & 57 & 54 & 50 & 52 & 53 & 47 & 8588 & 1.95 & 2.11 & 1.96 & 2.06 & 2.07 & 1.85 & 1.51\end{array}$ $\begin{array}{llllllllllllllll}738 & 75 & 74 & 70 & 67 & 61 & 62 & 59 & 11732 & 3.56 & 2.76 & 2.62 & 2.40 & 2.43 & 2.32 & 1.98\end{array}$ $\begin{array}{llllllllllllllll}742 & 74 & 74 & 70 & 66 & 61 & 60 & 55 & 11787 & 3.56 & 2.74 & 2.60 & 2.39 & 2.37 & 2.18 & 2.07\end{array}$

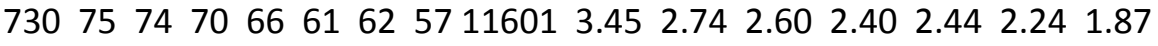

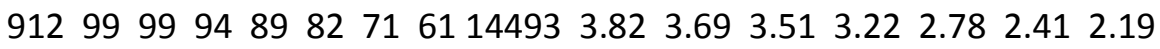

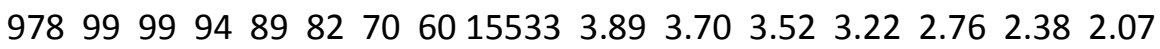

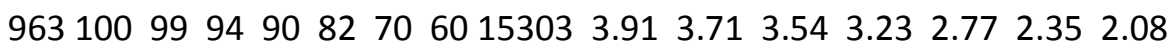
EOF 


\section{C.2 US 35 All Southbound Drops .FWD (.R80) File Format}

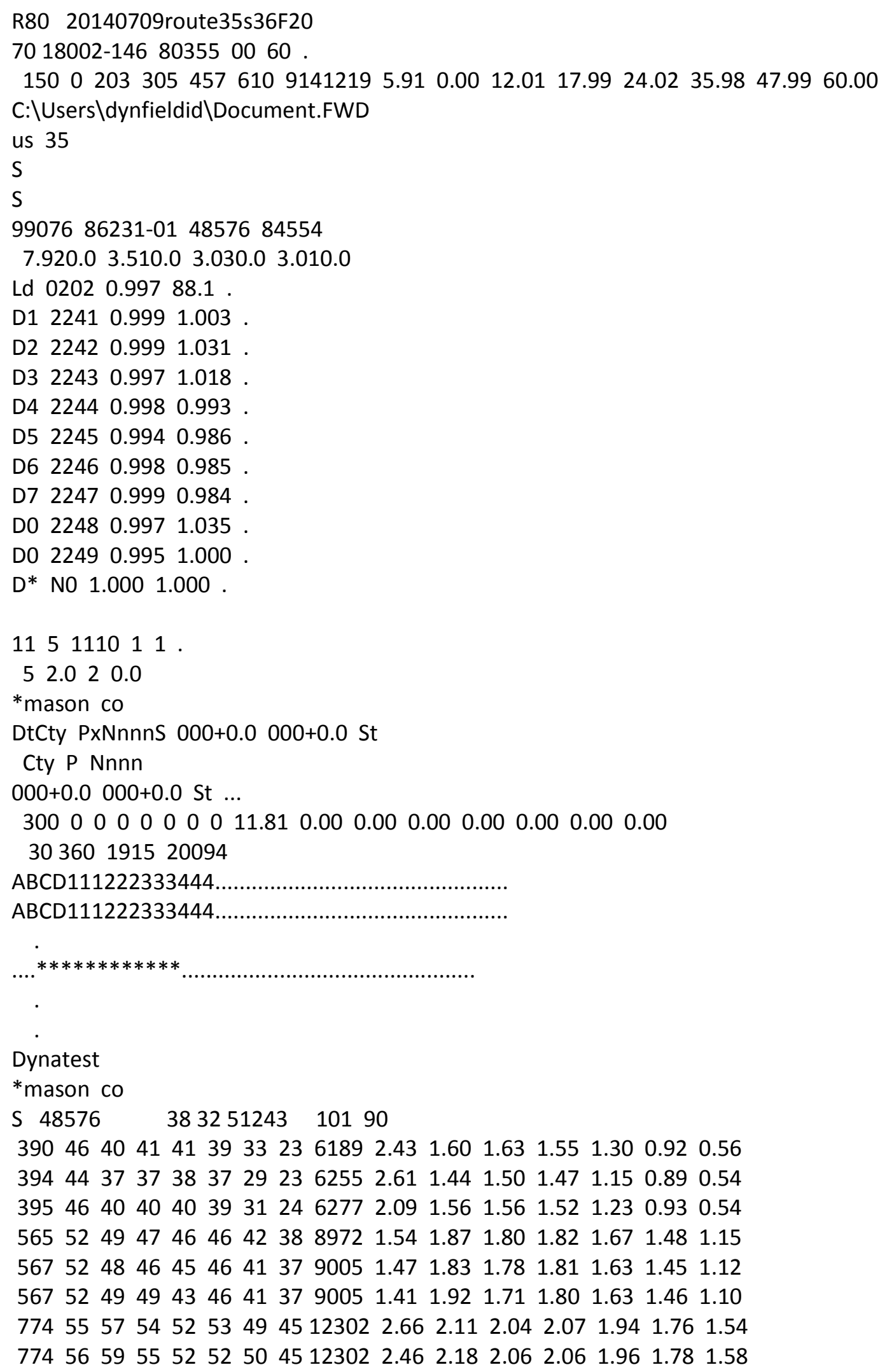




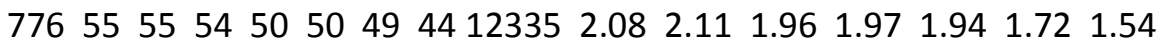
$\begin{array}{llllllllllllllll}998 & 65 & 67 & 64 & 58 & 54 & 49 & 46 & 15862 & 2.78 & 2.50 & 2.27 & 2.11 & 1.93 & 1.83 & 1.67\end{array}$

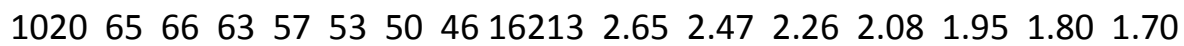
$\begin{array}{llllllllllllllll}983 & 65 & 67 & 63 & 57 & 52 & 50 & 46 & 15610 & 2.67 & 2.47 & 2.24 & 2.06 & 1.95 & 1.81 & 1.76\end{array}$ S $49093 \quad 393251246 \quad 10290$

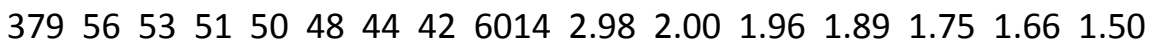
$\begin{array}{llllllllllllllll}381 & 56 & 53 & 51 & 49 & 49 & 45 & 42 & 6058 & 2.66 & 2.02 & 1.93 & 1.92 & 1.77 & 1.66 & 1.50\end{array}$

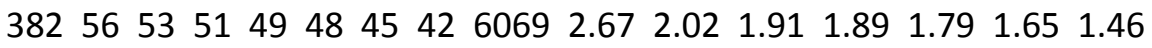
$\begin{array}{llllllllllllllll}544 & 81 & 76 & 74 & 69 & 64 & 53 & 47 & 8643 & 3.24 & 2.90 & 2.70 & 2.51 & 2.07 & 1.87 & 1.80\end{array}$ $\begin{array}{llllllllllllllll}547 & 81 & 76 & 73 & 68 & 64 & 53 & 48 & 8698 & 3.01 & 2.89 & 2.69 & 2.52 & 2.07 & 1.90 & 1.82\end{array}$

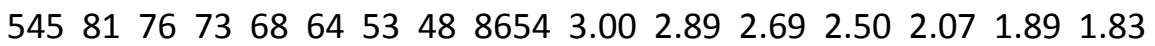

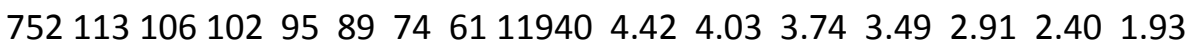

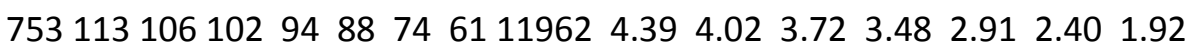

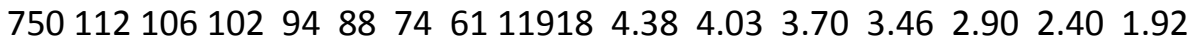
101415414313712811910083161035.945 .415 .024 .683 .953 .272 .62 $97715514413712811910183155225.82 \quad 5.405 .03 \quad 4.693 .963 .27 \quad 2.61$ 99915314213712711910083158735.805 .415 .014 .693 .953 .272 .60 S $49632 \quad 393251249 \quad 10190$

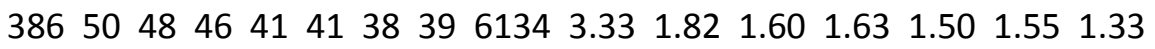
$\begin{array}{llllllllllllllll}388 & 51 & 49 & 48 & 43 & 43 & 40 & 40 & 6167 & 4.09 & 1.90 & 1.68 & 1.68 & 1.57 & 1.56 & 1.38\end{array}$ $\begin{array}{llllllllllllllll}382 & 50 & 48 & 47 & 42 & 43 & 41 & 39 & 6069 & 3.59 & 1.87 & 1.66 & 1.70 & 1.62 & 1.52 & 1.37\end{array}$ $\begin{array}{llllllllllllllll}558 & 71 & 69 & 68 & 62 & 60 & 52 & 46 & 8873 & 2.80 & 2.66 & 2.44 & 2.36 & 2.06 & 1.83 & 1.61\end{array}$ $\begin{array}{llllllllllllllll}558 & 72 & 70 & 68 & 64 & 61 & 53 & 46 & 8862 & 2.92 & 2.68 & 2.50 & 2.39 & 2.09 & 1.81 & 1.59\end{array}$

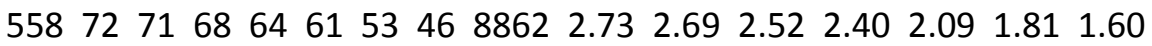
$\begin{array}{llllllllllllllll}760 & 101 & 99 & 95 & 85 & 74 & 64 & 12072 & 3.93 & 3.74 & 3.53 & 3.35 & 2.93 & 2.51 & 2.09\end{array}$

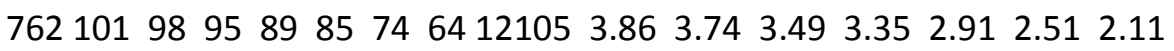

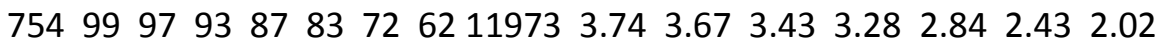
$101713513212711911410086161585.275 .014 .694 .493 .92 \quad 3.38 \quad 2.84$ 101713513212711811410087161585.315 .014 .664 .503 .923 .412 .90 $98113513212812011410086155885.205 .02 \quad 4.714 .503 .93 \quad 3.372 .83$ S $50149 \quad 3732512519990$

$\begin{array}{llllllllllllllll}385 & 37 & 36 & 36 & 33 & 31 & 24 & 15 & 6124 & 2.85 & 1.41 & 1.28 & 1.24 & 0.94 & 0.58 & 0.35\end{array}$ $\begin{array}{llllllllllllllll}381 & 40 & 36 & 38 & 34 & 31 & 24 & 15 & 6058 & 2.79 & 1.49 & 1.35 & 1.22 & 0.95 & 0.58 & 0.37\end{array}$ $\begin{array}{llllllllllllllll}386 & 39 & 36 & 38 & 35 & 33 & 24 & 15 & 6134 & 2.81 & 1.50 & 1.38 & 1.29 & 0.96 & 0.58 & 0.41\end{array}$ $\begin{array}{llllllllllllllll}551 & 50 & 50 & 49 & 50 & 48 & 37 & 34 & 8753 & 1.42 & 1.93 & 1.98 & 1.90 & 1.45 & 1.35 & 0.79\end{array}$ $\begin{array}{llllllllllllllll}552 & 52 & 51 & 50 & 52 & 50 & 38 & 35 & 8764 & 1.41 & 1.97 & 2.03 & 1.97 & 1.49 & 1.36 & 0.83\end{array}$ $\begin{array}{llllllllllllllll}552 & 51 & 50 & 50 & 50 & 50 & 39 & 34 & 8764 & 1.39 & 1.95 & 1.98 & 1.96 & 1.54 & 1.35 & 0.83\end{array}$ $\begin{array}{llllllllllllllll}757 & 56 & 52 & 54 & 54 & 52 & 50 & 39 & 12028 & 2.16 & 2.11 & 2.14 & 2.06 & 1.97 & 1.55 & 1.28\end{array}$

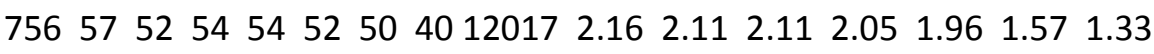
$\begin{array}{llllllllllllllll}761 & 57 & 53 & 55 & 54 & 54 & 52 & 40 & 12083 & 2.32 & 2.18 & 2.13 & 2.13 & 2.06 & 1.59 & 1.33\end{array}$ $\begin{array}{llllllllllllllll}977 & 66 & 66 & 64 & 58 & 54 & 48 & 45 & 15522 & 2.80 & 2.50 & 2.30 & 2.11 & 1.90 & 1.76 & 1.57\end{array}$ $\begin{array}{llllllllllllllll}939 & 66 & 66 & 63 & 58 & 54 & 48 & 45 & 14920 & 2.80 & 2.49 & 2.29 & 2.11 & 1.89 & 1.78 & 1.58\end{array}$ $\begin{array}{llllllllllllllll}973 & 67 & 66 & 63 & 58 & 54 & 47 & 45 & 15457 & 2.80 & 2.49 & 2.29 & 2.11 & 1.87 & 1.77 & 1.59\end{array}$ S $54886 \quad 373151256 \quad 9888$ $\begin{array}{llllllllllllllll}399 & 50 & 50 & 48 & 46 & 49 & 45 & 35 & 6332 & 4.32 & 1.90 & 1.83 & 1.91 & 1.77 & 1.39 & 1.36\end{array}$

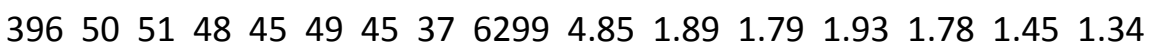

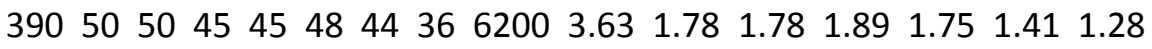
$\begin{array}{llllllllllllllll}528 & 61 & 61 & 57 & 56 & 53 & 52 & 45 & 8391 & 2.22 & 2.25 & 2.19 & 2.07 & 2.03 & 1.77 & 1.61\end{array}$ 
$\begin{array}{llllllllllllllll}532 & 61 & 60 & 57 & 56 & 52 & 51 & 46 & 8457 & 2.11 & 2.26 & 2.19 & 2.04 & 2.01 & 1.82 & 1.64\end{array}$ $\begin{array}{llllllllllllllll}531 & 60 & 61 & 57 & 56 & 52 & 51 & 44 & 8435 & 2.17 & 2.25 & 2.20 & 2.05 & 2.02 & 1.73 & 1.63\end{array}$ $\begin{array}{llllllllllllllll}730 & 85 & 83 & 81 & 77 & 71 & 61 & 51 & 11601 & 3.48 & 3.18 & 3.03 & 2.80 & 2.41 & 2.00 & 1.96\end{array}$ $\begin{array}{llllllllllllllll}747 & 85 & 84 & 81 & 77 & 72 & 61 & 52 & 11864 & 3.19 & 3.17 & 3.03 & 2.82 & 2.40 & 2.03 & 1.91\end{array}$ $\begin{array}{llllllllllllllll}726 & 84 & 83 & 80 & 76 & 71 & 60 & 51 & 11535 & 3.21 & 3.15 & 3.01 & 2.78 & 2.38 & 2.02 & 1.94\end{array}$ $961115113108103968167152704.514 .264 .07 \quad 3.78 \quad 3.172 .65 \quad 2.13$ 971116113108103958167154244.484 .274 .073 .743 .202 .642 .12 932116113108104968167147994.434 .274 .093 .773 .202 .652 .13 S $55392 \quad 383151258 \quad 10088$

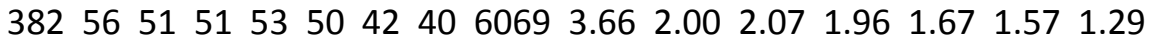

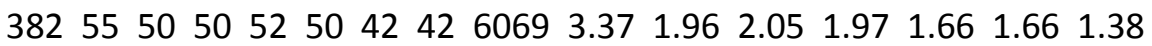
$\begin{array}{llllllllllllllll}376 & 54 & 50 & 50 & 52 & 51 & 42 & 39 & 5981 & 3.57 & 1.98 & 2.04 & 2.00 & 1.64 & 1.54 & 1.41\end{array}$ $\begin{array}{llllllllllllllll}543 & 63 & 58 & 57 & 60 & 59 & 56 & 54 & 8621 & 2.34 & 2.25 & 2.38 & 2.31 & 2.19 & 2.11 & 1.83\end{array}$ $\begin{array}{llllllllllllllll}543 & 65 & 60 & 58 & 61 & 59 & 57 & 54 & 8632 & 3.09 & 2.28 & 2.41 & 2.33 & 2.24 & 2.11 & 1.78\end{array}$ $\begin{array}{llllllllllllllll}547 & 62 & 58 & 57 & 60 & 60 & 56 & 53 & 8698 & 3.18 & 2.24 & 2.37 & 2.35 & 2.19 & 2.09 & 1.87\end{array}$ $\begin{array}{llllllllllllllll}735 & 86 & 81 & 75 & 71 & 68 & 61 & 59 & 11677 & 6.44 & 2.97 & 2.80 & 2.66 & 2.41 & 2.31 & 2.13\end{array}$ $\begin{array}{llllllllllllllll}741 & 87 & 81 & 76 & 71 & 68 & 61 & 59 & 11776 & 6.27 & 2.99 & 2.81 & 2.68 & 2.41 & 2.31 & 2.07\end{array}$

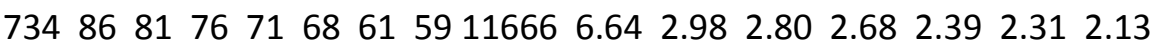

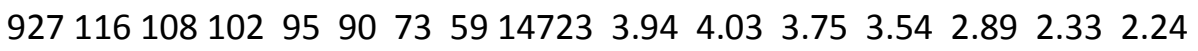
$\begin{array}{lllllllllllll}968 & 1151081039690 & 73 & 61 & 15380 & 3.80 & 4.04 & 3.76 & 3.53 & 2.89 & 2.40 & 2.27\end{array}$

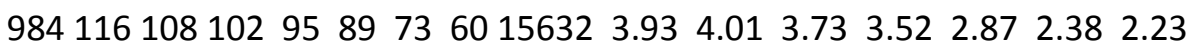
S $56955 \quad 393251301 \quad 10189$

$\begin{array}{llllllllllllllll}382 & 65 & 58 & 56 & 52 & 55 & 48 & 46 & 6069 & 2.60 & 2.19 & 2.06 & 2.16 & 1.89 & 1.83 & 1.51\end{array}$

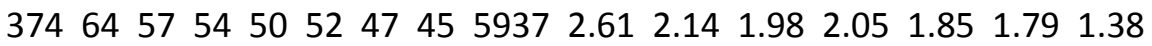

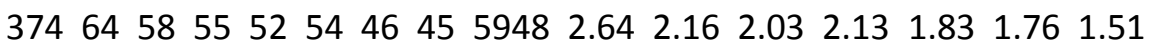
$\begin{array}{llllllllllllllll}543 & 91 & 83 & 78 & 72 & 65 & 56 & 53 & 8632 & 3.36 & 3.09 & 2.82 & 2.54 & 2.20 & 2.10 & 1.85\end{array}$ $\begin{array}{llllllllllllllll}544 & 91 & 83 & 79 & 72 & 65 & 54 & 53 & 8643 & 3.31 & 3.10 & 2.84 & 2.56 & 2.12 & 2.08 & 1.88\end{array}$ $\begin{array}{llllllllllllllll}543 & 91 & 82 & 78 & 72 & 65 & 54 & 53 & 8632 & 3.31 & 3.07 & 2.83 & 2.54 & 2.12 & 2.07 & 1.94\end{array}$

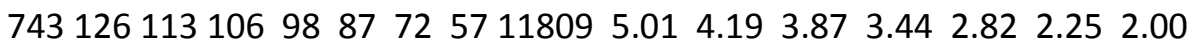

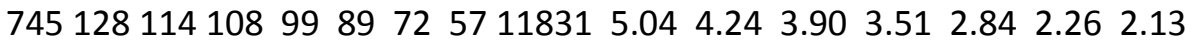

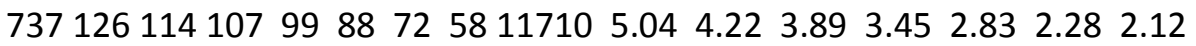
$9601701501431301199674152496.385 .635 .114 .67 \quad 3.78 \quad 2.93 \quad 2.21$ $9601701511431311179776152496.395 .635 .154 .593 .80 \quad 2.98 \quad 2.23$ 9741671501421301149676154686.335 .595 .134 .503 .773 .002 .25 S $57489 \quad 393251303 \quad 10290$

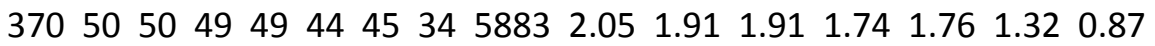
$\begin{array}{llllllllllllllll}370 & 47 & 52 & 47 & 50 & 45 & 44 & 34 & 5883 & 2.57 & 1.84 & 1.96 & 1.76 & 1.72 & 1.33 & 0.80\end{array}$ $\begin{array}{llllllllllllllll}372 & 46 & 48 & 46 & 48 & 43 & 43 & 32 & 5915 & 2.40 & 1.80 & 1.90 & 1.68 & 1.69 & 1.26 & 0.78\end{array}$ $\begin{array}{llllllllllllllll}545 & 54 & 56 & 55 & 55 & 53 & 51 & 44 & 8665 & 1.81 & 2.15 & 2.17 & 2.07 & 2.00 & 1.74 & 1.42\end{array}$ $\begin{array}{llllllllllllllll}546 & 62 & 61 & 61 & 60 & 60 & 56 & 48 & 8676 & 1.88 & 2.39 & 2.38 & 2.35 & 2.19 & 1.89 & 1.56\end{array}$ $\begin{array}{llllllllllllllll}547 & 58 & 58 & 56 & 57 & 55 & 53 & 46 & 8687 & 1.87 & 2.20 & 2.25 & 2.16 & 2.09 & 1.83 & 1.47\end{array}$ $\begin{array}{llllllllllllllll}749 & 74 & 73 & 70 & 67 & 66 & 56 & 53 & 11907 & 3.56 & 2.75 & 2.64 & 2.61 & 2.20 & 2.07 & 1.85\end{array}$

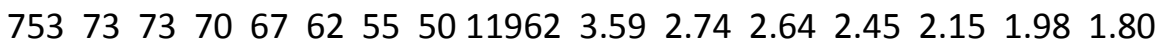

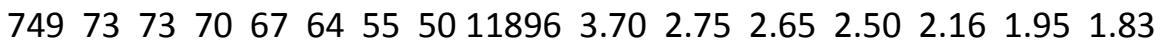

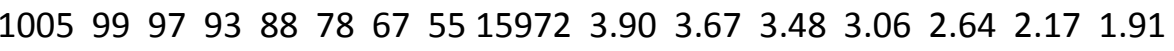

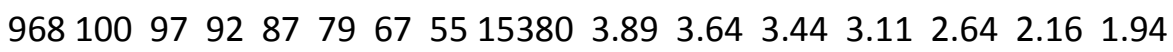

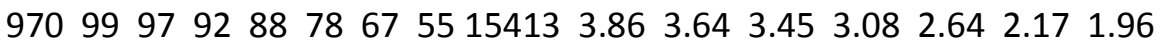
S $60076 \quad 403251307 \quad 10590$ 
$\begin{array}{llllllllllllllll}373 & 47 & 46 & 44 & 44 & 46 & 41 & 36 & 5926 & 2.31 & 1.75 & 1.72 & 1.81 & 1.62 & 1.43 & 1.24\end{array}$

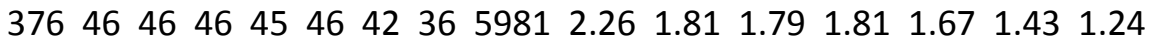
$\begin{array}{llllllllllllllll}377 & 47 & 46 & 45 & 46 & 46 & 44 & 36 & 5992 & 2.14 & 1.79 & 1.80 & 1.81 & 1.72 & 1.43 & 1.26\end{array}$ $\begin{array}{llllllllllllllll}546 & 68 & 66 & 64 & 61 & 57 & 50 & 47 & 8676 & 2.69 & 2.53 & 2.39 & 2.23 & 1.95 & 1.87 & 1.78\end{array}$ $\begin{array}{llllllllllllllll}551 & 68 & 66 & 64 & 61 & 57 & 50 & 49 & 8753 & 2.71 & 2.53 & 2.40 & 2.25 & 1.97 & 1.92 & 1.80\end{array}$

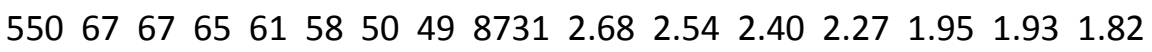

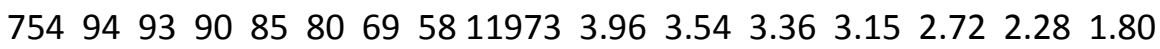
$\begin{array}{lllllllllllllllll}747 & 94 & 93 & 90 & 85 & 80 & 69 & 58 & 11875 & 3.96 & 3.54 & 3.36 & 3.14 & 2.70 & 2.30 & 1.81\end{array}$

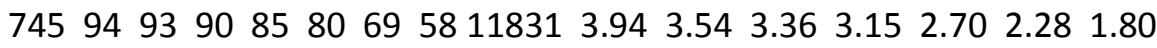
$9961271261221161109480158185.23 \quad 4.824 .574 .33 \quad 3.70 \quad 3.152 .51$ $9941261261221161109480157855.22 \quad 4.824 .57 \quad 4.32 \quad 3.703 .142 .51$ $9561271261231161109480151945.224 .834 .584 .33 \quad 3.713 .142 .52$ S $60604 \quad 413251309 \quad 10590$

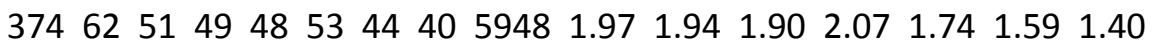

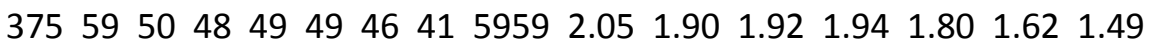
$\begin{array}{llllllllllllllll}377 & 58 & 53 & 49 & 49 & 50 & 47 & 41 & 5992 & 1.96 & 1.94 & 1.94 & 1.98 & 1.87 & 1.63 & 1.49\end{array}$ $\begin{array}{llllllllllllllll}541 & 79 & 69 & 64 & 63 & 63 & 58 & 55 & 8599 & 3.28 & 2.52 & 2.49 & 2.49 & 2.28 & 2.17 & 2.06\end{array}$ $\begin{array}{llllllllllllllll}539 & 78 & 69 & 65 & 64 & 65 & 60 & 56 & 8566 & 3.40 & 2.56 & 2.51 & 2.54 & 2.35 & 2.21 & 2.07\end{array}$ $\begin{array}{llllllllllllllll}539 & 78 & 69 & 65 & 64 & 61 & 60 & 57 & 8566 & 3.43 & 2.54 & 2.50 & 2.42 & 2.36 & 2.24 & 2.12\end{array}$

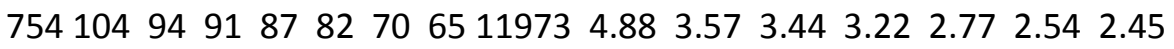

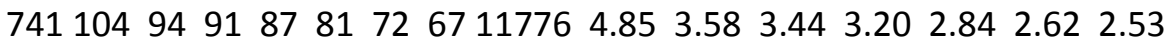
$\begin{array}{llllllllllllllll}743 & 103 & 94 & 92 & 88 & 82 & 73 & 67 & 11798 & 4.63 & 3.61 & 3.45 & 3.22 & 2.87 & 2.63 & 2.54\end{array}$ $9361351261211151099281148655.064 .784 .544 .283 .63 \quad 3.202 .94$ 9261371261201151099182147125.064 .734 .524 .303 .603 .242 .85 9551381251201151099183151725.004 .714 .524 .303 .583 .272 .86 S $61644 \quad 41335131210591$

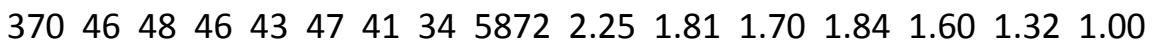

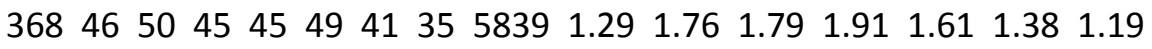
$\begin{array}{llllllllllllllll}372 & 46 & 48 & 45 & 44 & 49 & 40 & 36 & 5915 & 1.33 & 1.78 & 1.72 & 1.91 & 1.58 & 1.43 & 1.22\end{array}$ $\begin{array}{llllllllllllllll}541 & 52 & 51 & 49 & 51 & 52 & 50 & 44 & 8588 & 2.31 & 1.93 & 2.02 & 2.03 & 1.98 & 1.72 & 1.54\end{array}$ $\begin{array}{llllllllllllllll}539 & 55 & 53 & 52 & 53 & 52 & 51 & 46 & 8566 & 2.43 & 2.05 & 2.10 & 2.03 & 2.00 & 1.81 & 1.57\end{array}$ $\begin{array}{llllllllllllllll}539 & 54 & 52 & 50 & 52 & 54 & 50 & 44 & 8566 & 2.15 & 1.97 & 2.04 & 2.12 & 1.98 & 1.72 & 1.59\end{array}$ $\begin{array}{llllllllllllllll}742 & 71 & 72 & 70 & 66 & 61 & 55 & 52 & 11787 & 2.82 & 2.74 & 2.58 & 2.39 & 2.15 & 2.04 & 1.89\end{array}$

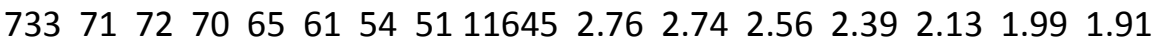

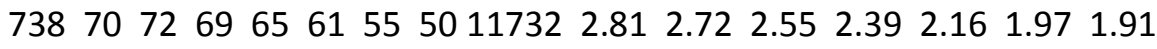

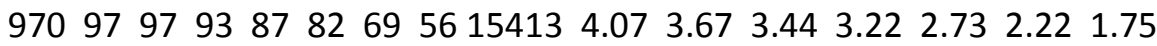

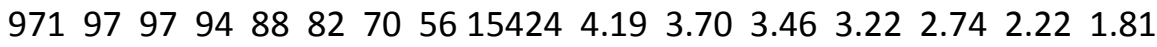

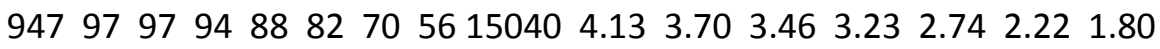
S $62172 \quad 413351314 \quad 10691$

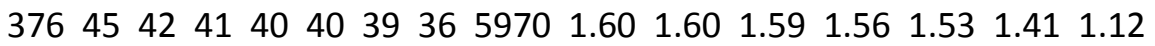

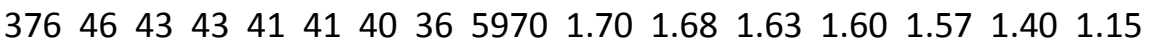
$\begin{array}{llllllllllllllll}373 & 44 & 41 & 41 & 41 & 40 & 39 & 36 & 5926 & 1.70 & 1.63 & 1.60 & 1.58 & 1.53 & 1.42 & 1.12\end{array}$ $\begin{array}{llllllllllllllll}538 & 51 & 50 & 48 & 47 & 47 & 45 & 43 & 8544 & 3.16 & 1.90 & 1.87 & 1.87 & 1.79 & 1.70 & 1.55\end{array}$ $\begin{array}{llllllllllllllll}543 & 51 & 50 & 49 & 49 & 49 & 46 & 43 & 8621 & 3.26 & 1.91 & 1.92 & 1.94 & 1.81 & 1.70 & 1.54\end{array}$ $\begin{array}{llllllllllllllll}543 & 51 & 50 & 49 & 47 & 48 & 45 & 43 & 8621 & 3.16 & 1.91 & 1.85 & 1.90 & 1.78 & 1.70 & 1.54\end{array}$

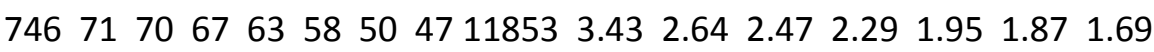

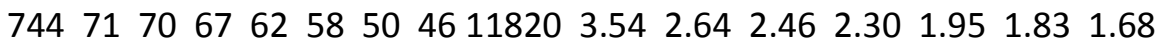

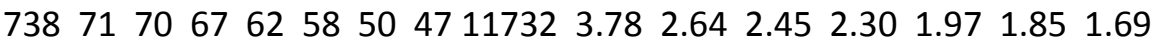




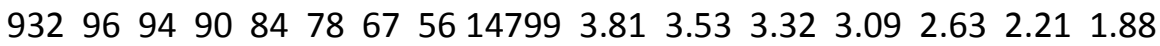
$\begin{array}{lllllllllllllll}1003 & 96 & 94 & 90 & 85 & 78 & 67 & 15939 & 3.86 & 3.54 & 3.33 & 3.09 & 2.63 & 2.20 & 1.84\end{array}$

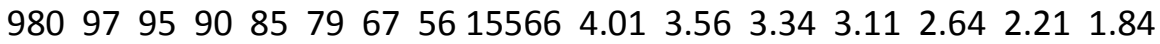
S $64506 \quad 423351317 \quad 10792$

$\begin{array}{llllllllllllllll}366 & 50 & 44 & 40 & 45 & 42 & 42 & 37 & 5817 & 1.08 & 1.57 & 1.79 & 1.67 & 1.64 & 1.44 & 1.24\end{array}$

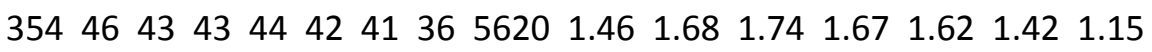
$\begin{array}{llllllllllllllll}354 & 42 & 45 & 43 & 46 & 40 & 42 & 38 & 5620 & 1.38 & 1.68 & 1.80 & 1.59 & 1.65 & 1.48 & 1.20\end{array}$ $\begin{array}{llllllllllllllll}521 & 55 & 57 & 60 & 55 & 51 & 54 & 49 & 8282 & 5.40 & 2.36 & 2.15 & 2.01 & 2.13 & 1.93 & 1.72\end{array}$ $\begin{array}{llllllllllllllll}523 & 55 & 56 & 55 & 54 & 49 & 53 & 47 & 8303 & 5.13 & 2.15 & 2.11 & 1.94 & 2.07 & 1.85 & 1.63\end{array}$ $\begin{array}{llllllllllllllll}524 & 56 & 56 & 58 & 53 & 50 & 54 & 47 & 8325 & 5.45 & 2.27 & 2.09 & 1.95 & 2.11 & 1.86 & 1.66\end{array}$ $\begin{array}{llllllllllllllll}718 & 78 & 77 & 75 & 71 & 67 & 62 & 57 & 11415 & 3.17 & 2.96 & 2.78 & 2.63 & 2.43 & 2.25 & 2.16\end{array}$

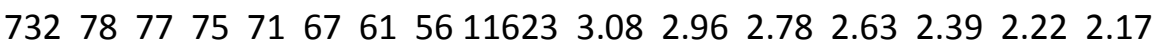

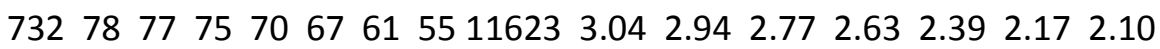
$91710310310095907866145695.963 .95 \quad 3.743 .543 .072 .612 .20$ $96310410310095907966152926.35 \quad 3.95 \quad 3.74 \quad 3.53 \quad 3.10 \quad 2.61 \quad 2.18$ $90610310310195897866143946.01 \quad 3.963 .743 .51 \quad 3.092 .612 .22$ S $65039 \quad 423351319 \quad 10891$

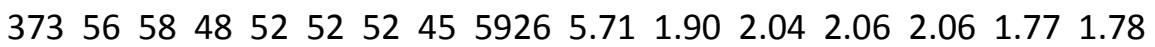
$\begin{array}{llllllllllllllll}369 & 53 & 58 & 50 & 51 & 53 & 51 & 45 & 5861 & 5.81 & 1.96 & 2.02 & 2.08 & 1.99 & 1.78 & 1.78\end{array}$

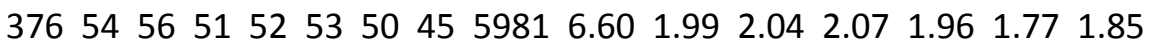
$\begin{array}{llllllllllllllll}537 & 62 & 64 & 61 & 58 & 62 & 59 & 53 & 8533 & 2.50 & 2.40 & 2.28 & 2.43 & 2.32 & 2.10 & 2.22\end{array}$ $\begin{array}{llllllllllllllll}538 & 62 & 64 & 62 & 59 & 63 & 59 & 55 & 8544 & 2.65 & 2.46 & 2.33 & 2.47 & 2.34 & 2.15 & 2.09\end{array}$ $\begin{array}{llllllllllllllll}539 & 60 & 64 & 64 & 59 & 63 & 58 & 52 & 8566 & 2.62 & 2.53 & 2.31 & 2.49 & 2.28 & 2.06 & 1.94\end{array}$ $\begin{array}{llllllllllllllll}746 & 81 & 84 & 82 & 75 & 73 & 66 & 56 & 11853 & 3.48 & 3.22 & 2.95 & 2.89 & 2.58 & 2.20 & 2.25\end{array}$

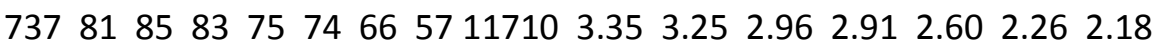
$\begin{array}{llllllllllllllll}745 & 81 & 85 & 82 & 75 & 74 & 65 & 57 & 11842 & 3.31 & 3.22 & 2.97 & 2.91 & 2.57 & 2.24 & 2.22\end{array}$ $989108111109101998573157094.434 .313 .973 .883 .35 \quad 2.87 \quad 2.37$ $94710811111010198 \quad 8673150404.364 .33 \quad 3.98 \quad 3.87 \quad 3.37 \quad 2.88 \quad 2.38$ $992108112110101998673157524.304 .32 \quad 3.98 \quad 3.893 .392 .88 \quad 2.45$ S $66338 \quad 443351322 \quad 11191$

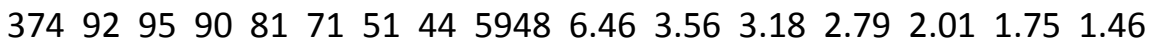
$\begin{array}{llllllllllllllll}381 & 93 & 95 & 90 & 81 & 71 & 51 & 45 & 6047 & 6.47 & 3.56 & 3.19 & 2.80 & 2.02 & 1.77 & 1.49\end{array}$

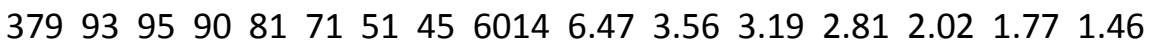

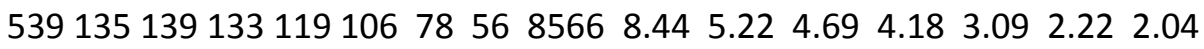

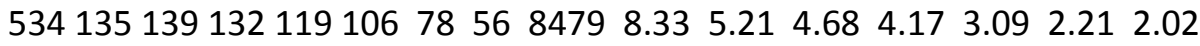
$\begin{array}{lllllllllll}536 & 13513913211910678568523 & 8.30 & 5.21 & 4.69 & 4.17 & 3.09 & 2.21 & 2.08\end{array}$ $741189195185167149111811176511.32 \quad 7.306 .585 .874 .37 \quad 3.172 .17$ $739190194185167149111811174311.307 .296 .58 \begin{array}{llllll}5.87 & 4.36 & 3.17 & 2.18\end{array}$ $734190195185167149111801166611.277 .306 .585 .874 .373 .16 \quad 2.26$ 9292582632502272031521111476715.139 .868 .927 .985 .974 .363 .03 9602582632512272031521111524915.069 .888 .948 .015 .984 .373 .03 9722582632512272031521111543514.979 .888 .948 .005 .994 .373 .04 S $66861 \quad 44335132411191$

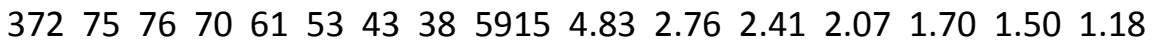

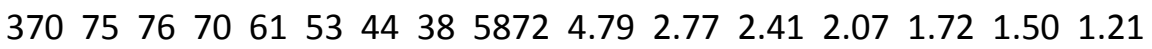

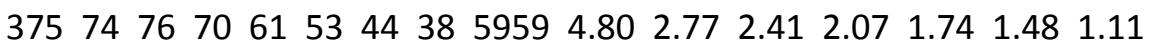

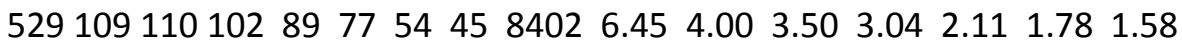

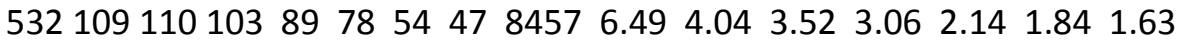




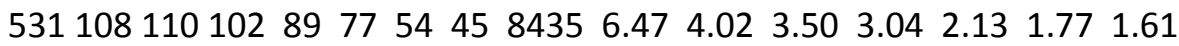
7361511531411241087552116888.665 .574 .894 .242 .962 .061 .76 $\begin{array}{lllllllllll}737150153142124108 & 76 & 5211710 & 8.60 & 5.58 & 4.90 & 4.25 & 2.98 & 2.03 & 1.74\end{array}$ 7351511531421241087652116778.575 .584 .904 .263 .002 .031 .72 $965205206191168146103711532512.007 .536 .605 .74 \quad 4.052 .811 .91$ 911204207192168146103711447111.917 .556 .635 .764 .072 .801 .93 $949204207192168146103711508411.917 .566 .635 .76 \quad 4.072 .791 .88$ S $69469 \quad 433351327 \quad 11091$

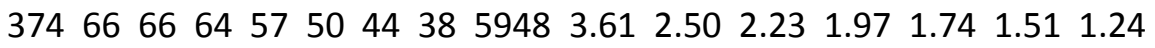

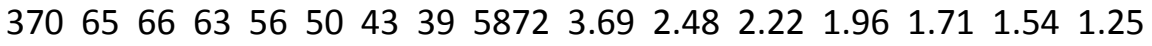
$\begin{array}{llllllllllllllll}372 & 65 & 66 & 63 & 56 & 50 & 44 & 39 & 5915 & 3.70 & 2.49 & 2.22 & 1.96 & 1.74 & 1.52 & 1.29\end{array}$

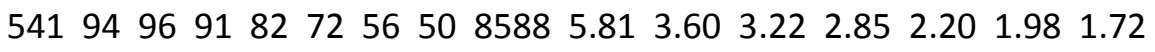
$\begin{array}{llllllllllllllll}541 & 93 & 95 & 91 & 82 & 72 & 57 & 51 & 8588 & 5.82 & 3.59 & 3.22 & 2.85 & 2.24 & 2.02 & 1.78\end{array}$

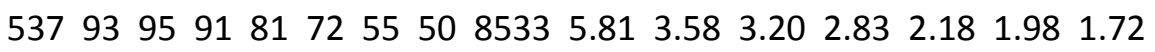
7411301321271131007560117657.715 .004 .453 .952 .942 .372 .11

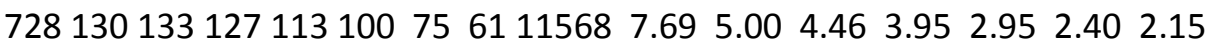
7361301331271131007559116887.695 .004 .463 .952 .952 .332 .12 973176178171153136102751545710.546 .736 .025 .344 .002 .962 .40 $928176178170152135101751474510.446 .716 .005 .32 \quad 3.982 .942 .44$ 901176178170152135101751431710.406 .716 .005 .323 .982 .942 .45 S $69992 \quad 42335133010791$

$\begin{array}{llllllllllllllll}368 & 82 & 86 & 80 & 72 & 62 & 45 & 41 & 5850 & 6.26 & 3.15 & 2.82 & 2.44 & 1.78 & 1.60 & 1.24\end{array}$ $\begin{array}{llllllllllllllll}370 & 83 & 87 & 81 & 72 & 62 & 45 & 40 & 5883 & 6.25 & 3.17 & 2.84 & 2.45 & 1.78 & 1.58 & 1.24\end{array}$ $\begin{array}{llllllllllllllll}368 & 83 & 86 & 80 & 72 & 62 & 46 & 41 & 5839 & 6.22 & 3.16 & 2.83 & 2.44 & 1.83 & 1.62 & 1.29\end{array}$ $53212112711810693664984467.834 .654 .173 .652 .61 \quad 1.941 .70$

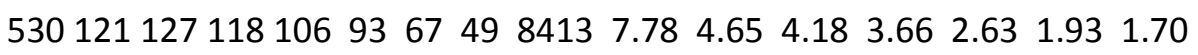

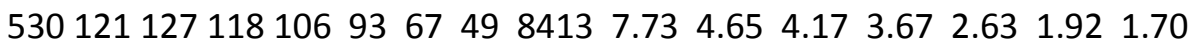
$73217117816614913194661162311.246 .53 \quad 5.885 .143 .72 \quad 2.611 .94$ $73117017816614913194661161211.216 .545 .885 .15 \quad 3.72 \quad 2.601 .93$ $72517117816614913194661151311.206 .545 .885 .143 .72 \quad 2.601 .96$ 862233243226203179130911369314.468 .898 .017 .045 .113 .572 .45 974234243226204179130911547914.408 .918 .037 .065 .133 .592 .46 $954234243226204179130911515014.348 .918 .03 \quad 7.05 \quad 5.13 \quad 3.592 .45$ S $72574 \quad 413351333 \quad 10591$

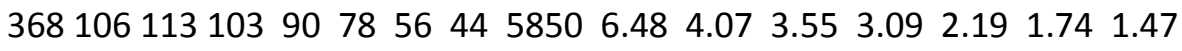
$3701061131049079564358836.414 .08 \quad 3.56 \quad 3.10 \quad 2.201 .691 .46$

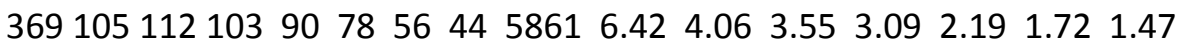
$\begin{array}{llllllllllll}543152 & 16314913211582 & 57 & 8621 & 7.62 & 5.87 & 5.19 & 4.54 & 3.24 & 2.23 & 1.73\end{array}$ $\begin{array}{lllllllllll}536153163150132116 & 82 & 57 & 8523 & 7.59 & 5.89 & 5.20 & 4.55 & 3.24 & 2.25 & 1.72\end{array}$

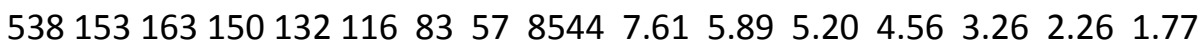
$735210224206183161116811167710.878 .117 .206 .34 \quad 4.563 .172 .20$ $728211225207183161116811156810.948 .137 .206 .34 \quad 4.573 .172 .19$ 736210224206182160116801168810.858 .107 .186 .314 .553 .162 .18 9642822982742442151561091531414.5510 .809 .598 .476 .164 .312 .99 9632832992752452161571091530314.5110 .839 .638 .506 .184 .312 .99 9312822982742442151561091478814.4310 .809 .598 .476 .154 .302 .98 S $73117 \quad 413351335 \quad 10691$

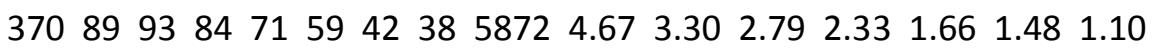




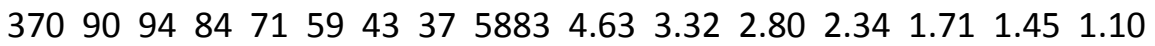

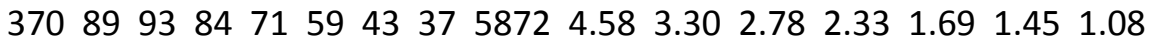

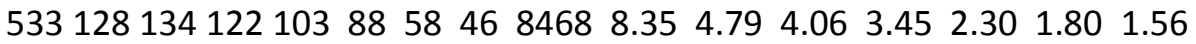

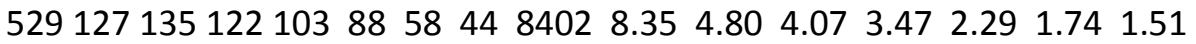
$5291271341211038858458402 \quad 8.31 \quad 4.77 \quad 4.06 \quad 3.45 \quad 2.28 \quad 1.76 \quad 1.57$ $7261761821661421218153115359.816 .525 .584 .76 \quad 3.202 .07 \quad 1.80$ 7271751831661421218153115579.716 .525 .584 .763 .192 .081 .77 7321751821651411208153116349.716 .495 .564 .743 .202 .081 .80 $954233242219188161109721515013.658 .637 .416 .34 \quad 4.30 \quad 2.832 .04$ $867233242220188161109721378113.53 \quad 8.657 .42 \quad 6.354 .30 \quad 2.842 .07$ $970233242220188162109721541313.508 .657 .426 .36 \quad 4.28 \quad 2.842 .02$ S $75705 \quad 423351338 \quad 10891$

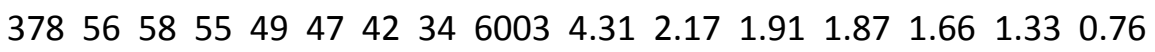

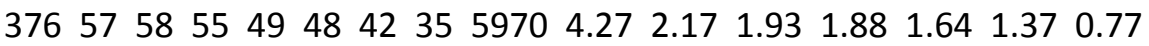
$\begin{array}{llllllllllllllll}375 & 58 & 58 & 55 & 49 & 48 & 42 & 35 & 5959 & 4.29 & 2.17 & 1.93 & 1.88 & 1.66 & 1.36 & 0.80\end{array}$ $\begin{array}{llllllllllllllll}532 & 84 & 86 & 81 & 72 & 63 & 52 & 44 & 8457 & 6.50 & 3.20 & 2.83 & 2.47 & 2.03 & 1.73 & 1.46\end{array}$ $\begin{array}{llllllllllllllll}530 & 85 & 86 & 82 & 72 & 63 & 52 & 45 & 8424 & 6.48 & 3.21 & 2.85 & 2.47 & 2.04 & 1.77 & 1.46\end{array}$ $\begin{array}{llllllllllllllll}532 & 85 & 86 & 81 & 71 & 62 & 51 & 45 & 8446 & 6.41 & 3.19 & 2.81 & 2.44 & 2.00 & 1.78 & 1.43\end{array}$ $\begin{array}{llllllllllll}732 & 11712011410188 & 61 & 51 & 11634 & 8.56 & 4.47 & 3.97 & 3.46 & 2.40 & 2.02 & 1.81\end{array}$ $\begin{array}{llllllllllll}73811612011310188 & 61 & 51 & 11721 & 8.55 & 4.46 & 3.96 & 3.45 & 2.39 & 2.00 & 1.76\end{array}$ $\begin{array}{lllllllllll}73711712011310188 & 61 & 111710 & 8.50 & 4.46 & 3.96 & 3.46 & 2.39 & 2.00 & 1.77\end{array}$ $97616316515513812084571551111.476 .125 .43 \quad 4.74 \quad 3.302 .24 \quad 2.02$ $97216216615613812184571543511.376 .135 .44 \quad 4.75 \quad 3.312 .252 .00$ $94916316615613912184571508411.356 .155 .46 \quad 4.76 \quad 3.312 .26 \quad 2.04$ S $76233 \quad 433351340 \quad 10991$

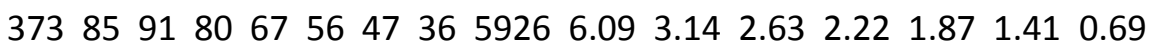
$\begin{array}{llllllllllllllll}372 & 84 & 90 & 80 & 66 & 57 & 48 & 36 & 5915 & 6.05 & 3.13 & 2.60 & 2.23 & 1.88 & 1.42 & 0.70\end{array}$

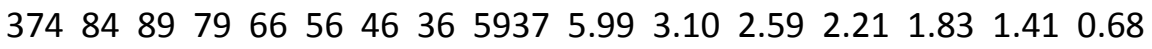

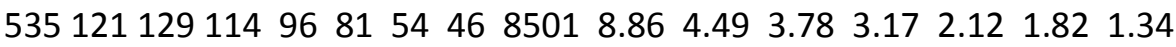

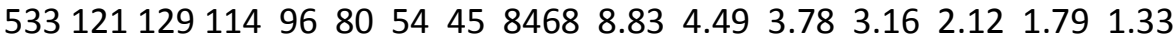

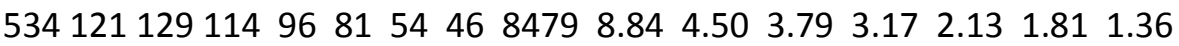
72617018216213711675501153511.816 .365 .414 .562 .951 .971 .66 $73217018216213711675491163411.746 .365 .414 .56 \quad 2.941 .941 .64$ $73017018216213811675491160111.726 .375 .43 \quad 4.562 .961 .941 .65$ 947233247220188159104651504015.748 .677 .396 .264 .092 .551 .78 $919233247220187158104651460215.618 .657 .376 .24 \quad 4.10 \quad 2.541 .78$ 934233248220188159104651484315.578 .677 .396 .264 .092 .541 .77 S $76740 \quad 423251342 \quad 10790$

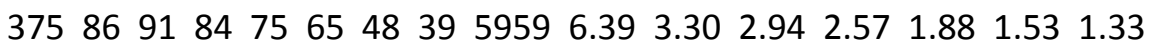
$\begin{array}{llllllllllllllll}376 & 85 & 90 & 84 & 74 & 65 & 48 & 39 & 5981 & 6.36 & 3.29 & 2.93 & 2.56 & 1.89 & 1.52 & 1.34\end{array}$

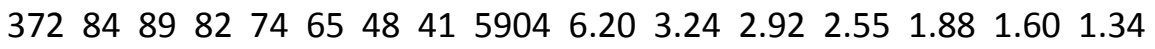

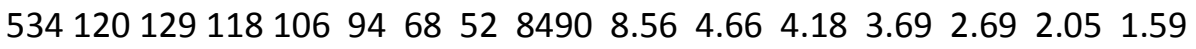

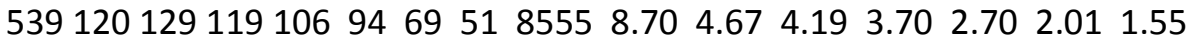

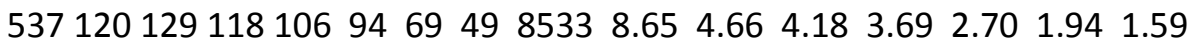
$73416717716314713095691165511.476 .435 .785 .113 .752 .73 \quad 1.88$ $73416617716314612995701165511.43 \quad 6.41 \quad 5.76 \quad 5.093 .73 \quad 2.741 .89$ $72916617716314713096691157911.376 .425 .775 .10 \quad 3.76 \quad 2.731 .88$ 914224238219197174128931451515.268 .637 .776 .875 .043 .662 .62 
963224239219198175129941529215.218 .637 .786 .895 .063 .692 .64 964224239219198175129941531415.178 .647 .796 .895 .073 .702 .66 S $77273 \quad 423251345 \quad 10790$

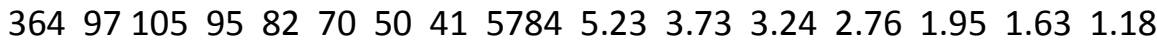

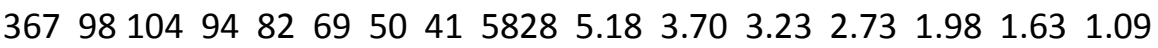

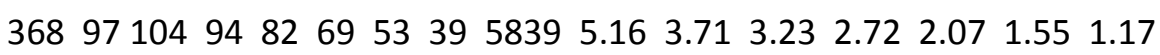
529138149136119102715384027.915 .344 .674 .032 .792 .071 .79

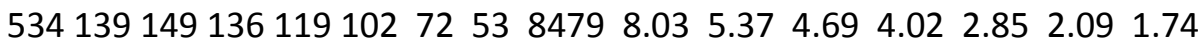
$\begin{array}{llllllllll}533 & 13814913611910173518468 & 7.92 & 5.35 & 4.68 & 3.99 & 2.87 & 2.02 & 1.80\end{array}$ $729190203186164141101701159010.767 .326 .455 .573 .96 \quad 2.741 .92$ $727191204186164142100701155710.837 .316 .455 .603 .93 \quad 2.741 .89$ 723191204186164142100701149110.807 .336 .465 .613 .952 .761 .85 $983254272248220192135961561014.419 .768 .667 .575 .33 \quad 3.762 .54$ $937254271247219191135961488714.299 .748 .637 .535 .33 \quad 3.762 .48$ $972254272247219192135961543514.269 .738 .637 .565 .33 \quad 3.762 .48$ S $79855 \quad 423351347 \quad 10791$

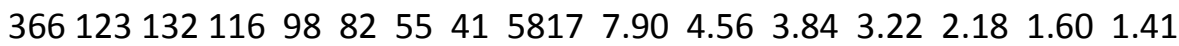

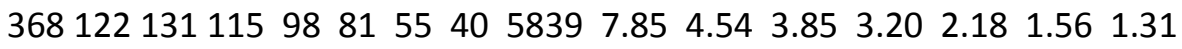

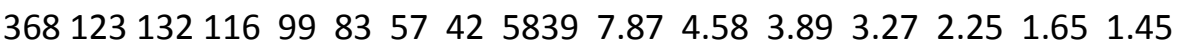
5271771891671421208256838011.096 .575 .594 .743 .242 .201 .68 5271771881661411198255836911.016 .545 .574 .703 .232 .171 .56

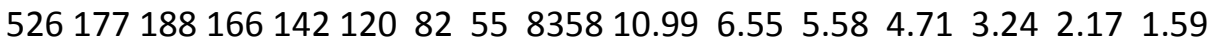
719245258230197166115791142514.709 .057 .756 .544 .523 .112 .09 $718246259230197167115781140414.709 .067 .766 .574 .52 \quad 3.092 .10$ 723245259230197167116781149114.689 .067 .776 .594 .553 .092 .09 9123323483102662261571071449319.5412 .2010 .478 .916 .194 .212 .83 $9513333483102662271571061510619.4412 .2110 .48 \quad 8.936 .184 .192 .81$ $9373333493102662271571061488719.3512 .2210 .498 .92 \quad 6.184 .182 .82$ S $80388 \quad 423351349 \quad 10792$

$\begin{array}{llllllllllllllll}364 & 51 & 49 & 47 & 45 & 40 & 21 & 9 & 5784 & 4.24 & 1.86 & 1.76 & 1.56 & 0.82 & 0.34 & 0.14\end{array}$ $\begin{array}{llllllllllllllll}369 & 48 & 49 & 47 & 45 & 39 & 21 & 7 & 5861 & 4.21 & 1.87 & 1.76 & 1.55 & 0.84 & 0.27 & 0.13\end{array}$ $\begin{array}{llllllllllllllll}370 & 49 & 50 & 48 & 45 & 39 & 22 & 7 & 5883 & 4.22 & 1.90 & 1.78 & 1.55 & 0.88 & 0.27 & 0.13\end{array}$ $\begin{array}{llllllllllllllll}527 & 62 & 68 & 58 & 56 & 52 & 37 & 22 & 8380 & 6.47 & 2.30 & 2.19 & 2.03 & 1.46 & 0.87 & 0.33\end{array}$ $\begin{array}{llllllllllllllll}527 & 62 & 69 & 58 & 55 & 51 & 36 & 21 & 8380 & 6.43 & 2.30 & 2.15 & 2.00 & 1.43 & 0.81 & 0.28\end{array}$ $\begin{array}{llllllllllllllll}527 & 62 & 69 & 59 & 55 & 50 & 37 & 21 & 8380 & 6.45 & 2.31 & 2.15 & 1.97 & 1.44 & 0.83 & 0.30\end{array}$ $\begin{array}{llllllllllllllll}721 & 85 & 93 & 80 & 63 & 55 & 45 & 34 & 11447 & 8.07 & 3.14 & 2.48 & 2.17 & 1.76 & 1.34 & 0.75\end{array}$ $\begin{array}{llllllllllllllll}728 & 85 & 94 & 80 & 64 & 55 & 45 & 34 & 11568 & 8.04 & 3.16 & 2.50 & 2.15 & 1.79 & 1.35 & 0.78\end{array}$

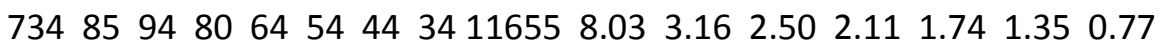
$894115126108866850401420810.734 .263 .40 \quad 2.691 .96 \quad 1.57 \quad 1.24$ 933116127108866849391482110.704 .263 .392 .691 .941 .541 .18 $888115127108866853401410910.674 .263 .402 .692 .08 \quad 1.571 .22$ S $80895 \quad 423351352 \quad 10892$

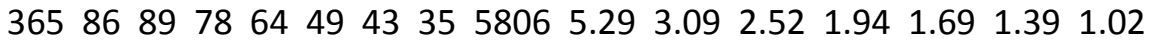

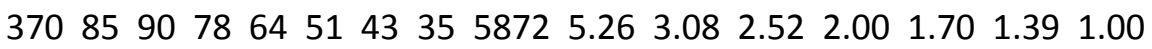

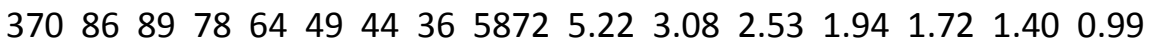

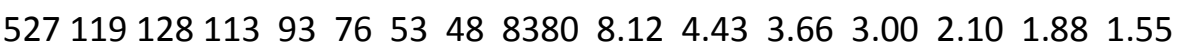

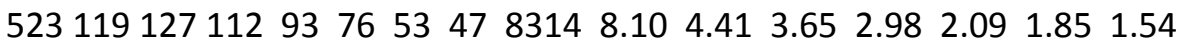

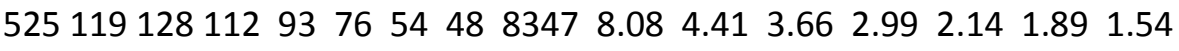


$72316517615613010767521149110.43 \quad 6.135 .114 .202 .652 .061 .83$ $72916517615613010667511157910.366 .135 .114 .192 .64 \quad 2.021 .81$

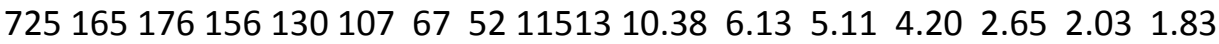
$97922423621017614592591555513.798 .286 .93 \quad 5.70 \quad 3.64 \quad 2.33 \quad 1.95$ $91222423621117614593591449313.808 .296 .945 .72 \quad 3.652 .331 .96$ $94922623621017614592591507313.72 \quad 8.286 .945 .693 .64 \quad 2.33 \quad 2.02$ S $81423 \quad 443351354 \quad 11092$

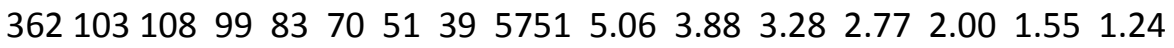

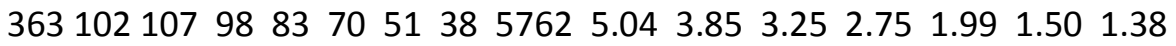

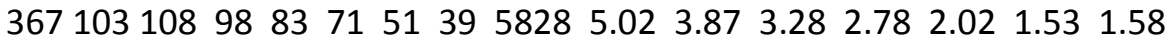
526149156142122104755183588.225 .604 .794 .102 .972 .021 .95

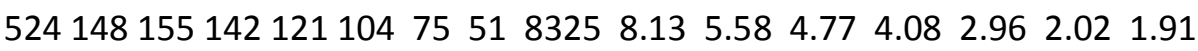

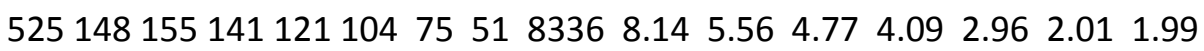
$724205214196169146106731150210.357 .716 .675 .754 .16 \quad 2.872 .44$ $734205214196170146106731166610.367 .736 .685 .764 .16 \quad 2.862 .46$ $724204213196169146105731150210.327 .706 .675 .74 \quad 4.152 .862 .45$ 9582762882642301991441001522714.6610 .399 .047 .835 .683 .932 .86 9312772892652302001451001478814.6310 .429 .077 .875 .693 .952 .89 9732772892652312001451001545714.5910 .439 .087 .875 .723 .952 .89 S $84036 \quad 433351357 \quad 11092$

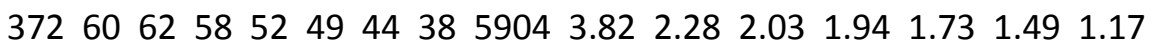
$\begin{array}{llllllllllllllll}373 & 60 & 62 & 57 & 51 & 48 & 43 & 37 & 5926 & 3.82 & 2.26 & 2.02 & 1.90 & 1.70 & 1.45 & 1.14\end{array}$ $\begin{array}{llllllllllllllll}370 & 59 & 62 & 58 & 51 & 48 & 44 & 38 & 5872 & 3.84 & 2.27 & 2.02 & 1.88 & 1.74 & 1.48 & 1.19\end{array}$ $\begin{array}{llllllllllllllll}536 & 85 & 89 & 84 & 75 & 66 & 50 & 45 & 8523 & 5.99 & 3.31 & 2.96 & 2.60 & 1.95 & 1.76 & 1.54\end{array}$ $\begin{array}{llllllllllllllll}537 & 85 & 88 & 84 & 75 & 66 & 50 & 45 & 8533 & 5.93 & 3.30 & 2.96 & 2.59 & 1.96 & 1.76 & 1.54\end{array}$ $\begin{array}{llllllllllllllll}535 & 85 & 88 & 84 & 75 & 66 & 50 & 45 & 8501 & 5.88 & 3.29 & 2.95 & 2.58 & 1.95 & 1.77 & 1.54\end{array}$ $734121126120108957051116667.794 .714 .243 .73 \quad 2.741 .991 .76$ $\begin{array}{lllllllllll}736122 & 1261201089570 & 51 & 11688 & 7.70 & 4.71 & 4.25 & 3.74 & 2.76 & 2.00 & 1.77\end{array}$ 725121126120108957050115247.674 .714 .253 .742 .751 .981 .82 $97216717416514913297711543510.786 .485 .855 .18 \quad 3.83 \quad 2.782 .02$ 90016717316514913298711429610.676 .505 .865 .193 .852 .782 .06 90316717316514913298711435010.656 .505 .875 .203 .842 .792 .01 S $84554 \quad 423351400 \quad 10791$

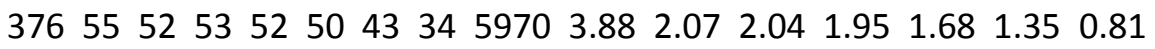
$\begin{array}{llllllllllllllll}375 & 54 & 52 & 53 & 51 & 48 & 42 & 35 & 5959 & 3.78 & 2.10 & 2.02 & 1.90 & 1.66 & 1.39 & 0.86\end{array}$ $\begin{array}{llllllllllllllll}375 & 53 & 53 & 54 & 52 & 50 & 43 & 35 & 5959 & 3.79 & 2.12 & 2.05 & 1.96 & 1.69 & 1.39 & 0.85\end{array}$

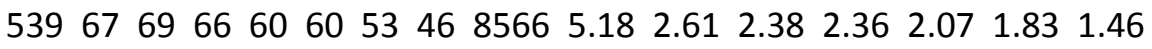

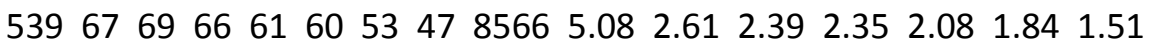

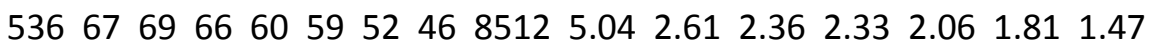

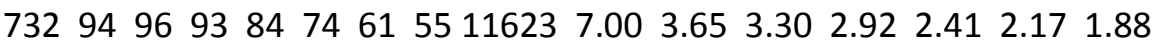
$\begin{array}{llllllllllllllll}729 & 94 & 96 & 93 & 83 & 74 & 61 & 55 & 11579 & 6.92 & 3.65 & 3.28 & 2.92 & 2.39 & 2.16 & 1.85\end{array}$

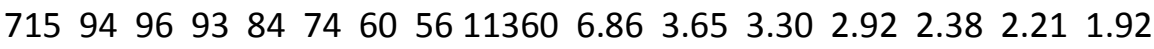
$9501291311261141017460150958.994 .974 .483 .982 .93 \quad 2.372 .12$ 9151291311261141017461145378.924 .974 .493 .992 .932 .392 .17 $9651291311261141017460153368.854 .974 .493 .992 .93 \quad 2.382 .17$ S $86592 \quad 393451416 \quad 10293$

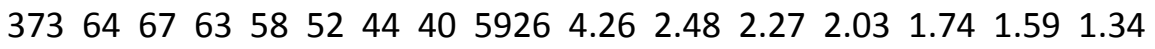

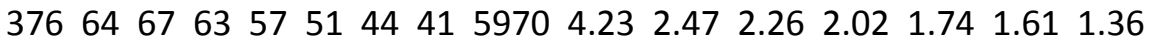




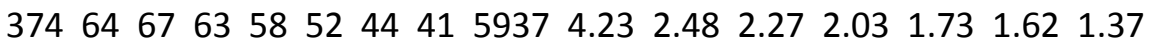

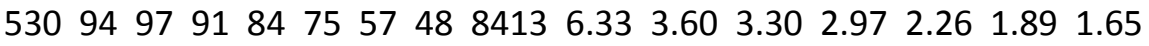

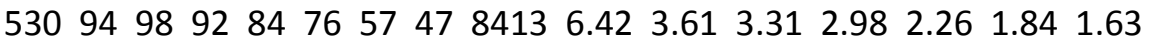

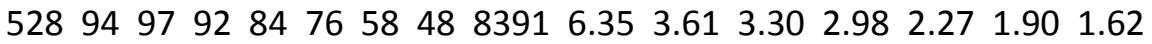

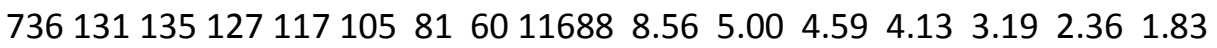
7431311351271171058160117988.565 .004 .594 .153 .202 .371 .83 $\begin{array}{llllllllllll}736131 & 135127117105816011688 & 8.57 & 5.01 & 4.60 & 4.14 & 3.20 & 2.37 & 1.86\end{array}$ $973178183172157142110821545711.136 .766 .205 .594 .34 \quad 3.22 \quad 2.33$ $970178183172158143110821541311.06 \quad 6.796 .22 \quad 5.62 \quad 4.33 \quad 3.23 \quad 2.34$ $940179183172158143110821493111.126 .786 .225 .62 \quad 4.33 \quad 3.232 .34$ S $87115 \quad 443451418 \quad 11193$

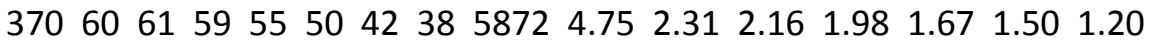
$\begin{array}{llllllllllllllll}368 & 59 & 61 & 59 & 55 & 51 & 41 & 37 & 5839 & 4.71 & 2.33 & 2.17 & 2.00 & 1.63 & 1.46 & 1.19\end{array}$

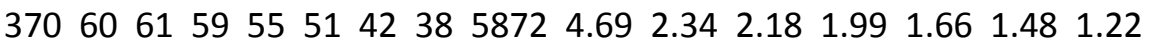
$\begin{array}{llllllllllllllll}532 & 87 & 89 & 86 & 80 & 73 & 58 & 49 & 8457 & 5.66 & 3.39 & 3.14 & 2.89 & 2.30 & 1.91 & 1.64\end{array}$ $\begin{array}{lllllllllllllllll}528 & 87 & 89 & 86 & 80 & 73 & 58 & 49 & 8391 & 5.55 & 3.38 & 3.13 & 2.88 & 2.28 & 1.92 & 1.68\end{array}$ $\begin{array}{llllllllllllllll}529 & 87 & 89 & 86 & 80 & 73 & 58 & 49 & 8402 & 5.52 & 3.39 & 3.14 & 2.88 & 2.28 & 1.91 & 1.67\end{array}$ $7311201231191101018163116127.84 \quad 4.674 .33 \quad 3.983 .182 .491 .93$ 7321201231191101028163116347.824 .674 .344 .003 .192 .491 .93 7301201231191101018163116017.794 .674 .323 .983 .182 .491 .89 927162166160148136109851472310.186 .305 .835 .364 .283 .332 .52 $931162166160149136109851478810.136 .305 .855 .374 .30 \quad 3.352 .54$ 976161165160148136108851550010.046 .285 .835 .354 .273 .332 .52 EOF 